\title{
Documentação e testes da biblioteca genérica de álgebra linear Klein
}

\author{
Rafael Freitas Schmid
}

\author{
DisSERTAÇÃO APRESENTADA \\ $\mathrm{AO}$ \\ Instituto DE MatemáticA E EstatísticA \\ DA \\ UNiversidade DE SÃo PAUlo \\ PARA \\ OBTENÇÃO DO TÍTULO \\ $\mathrm{DE}$ \\ Mestre EM CIÊNCIAS
}

Programa: Programa de Pós-Graduação em Ciências da Computação Orientador: Prof. Dr. Walter Figueiredo Mascarenhas 


\section{Documentação e testes da biblioteca \\ de programação linear Klein}

Esta é a versão original da dissertação elaborada pelo candidato Rafael Freitas Schmid, tal como submetida à Comissão Julgadora. 


\section{Resumo}

SCHMID, R. F. Documentação e testes da biblioteca de álgebra linear Klein. 2014. 120

f. Defesa da Dissertação - Instituto de Matemática e Estatística, Universidade de São Paulo, São Paulo, 2014.

Este trabalho descreve a Klein, uma biblioteca genérica para álgebra linear em $\mathrm{C}++$. A Klein facilita o uso de matrizes e vetores, permitindo que o usuário programe de modo similar ao Matlab. Com ela podemos, por exemplo, implementar um passo do método de Newton para a função $f$, através da expressão

$$
x=x-i n v(j a c(x)) * f(x),
$$

onde x é vetor, jac a Jacobiana e inv a inversa. Além disso, por se tratar de uma biblioteca genérica, os tipos envolvidos nestas expressões podem ser escolhidos pelo programador. O trabalho também discute como a biblioteca é testada, tanto do ponto de vista de corretude quanto de desempenho.

Palavras-chave: programação linear, teste de software, Klein, template, $\mathrm{c}++$, programação genérica, problemas de otimização, teste genérico, teste unitário. 


\section{Abstract}

SCHMID, R. F. Tests and documentation of the Klein library. 2014. 120 f. Masters Thesis Instituto de Matemática e Estatística, Universidade de São Paulo, São Paulo, 2014.

We describe the Klein library, a generic libray for linear algebra in $\mathrm{C}++$. It simplifies the use of vectors and matrices and let the user program as in Matlab. With Klein, one can for instance implement Newton's method as

$$
x=x-i n v(j a c) * f(x),
$$

where $x$ is a vector, $j a c$ is the Jacobian matrix, $i n v$ is the inverse operator and $f(x)$ is the function of which we want to find zero. Moreover, Klein is generic in the sense that it allows the use of arbitrary types of scalars (float, double, intervals, rationals, etc). We also explain how it is tested, both for correctness and performance.

Keywords: linear program, software testing, Klein, template, generic program, $\mathrm{c}++$, generic tests, unit tests. 


\section{Sumário}

Lista de Tabelas $\quad$ vii

1 Introdução $\quad 1$

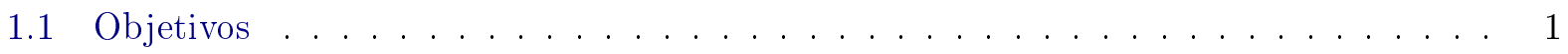

1.2 Organização do Trabalho . . . . . . . . . . . . . . . . . 1

2 Programação Genérica $\quad 3$

2.1 Templates em $\mathrm{C}++\ldots \ldots \ldots \ldots \ldots \ldots \ldots$

2.1 .1 Funções Template . . . . . . . . . . . . . . . . . . . . 3

2.1 .2 Classes Template . . . . . . . . . . . . . . . . . . . 5

2.1.3 Especialização de Templates . . . . . . . . . . . . . . . . . . . 5

2.2 Tipos genéricos de números utilizando templates $\ldots \ldots \ldots \ldots$

3 Arquitetura e estratégias da Klein $\quad 11$

3.1 Estratégias para avaliar expressões . . . . . . . . . . . . . . . . . 12

3.1 .1 Estratégia Lazy na Klein . . . . . . . . . . . . . . . . . . . . . 13

3.2 Alias . . . . . . . . . . . . . . . . . . . . . . 14

3.2 .1 Alias na Klein . . . . . . . . . . . . . . . . . . . . 16

3.3 Classes Traits . . . . . . . . . . . . . . . . . . . . 18

3.3 .1 Classes Traits da Klein . . . . . . . . . . . . . . . . . . . . . . 19

3.3.2 Exemplos de classes traits em funções . . . . . . . . . . . . . . . . 20

4 Objetos da Klein $\quad 21$

4.1 Escalares . . . . . . . . . . . . . . . . . . . . . 21

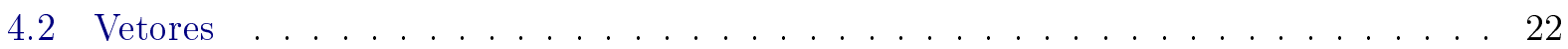

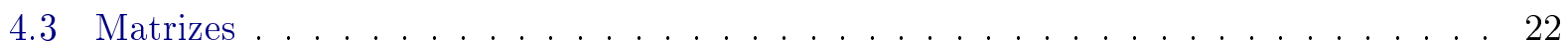

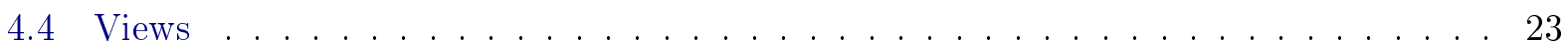

4.5 Expressões Aritméticas . . . . . . . . . . . . . . . . . . . . . . . . . 24

4.6 Operadores Lineares . . . . . . . . . . . . . . . . . 25

4.7 Decomposições . . . . . . . . . . . . . . . . . . . . . . . . . 29

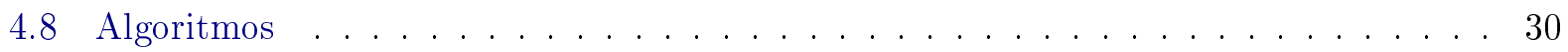

5 Algoritmos e funções da Klein $\quad 31$

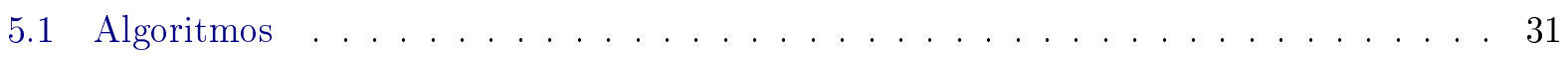

5.1 .1 Algoritmos da STL . . . . . . . . . . . . . . . . . . 31

5.1 .2 Algoritmos de Estatística . . . . . . . . . . . . . . 34

5.1 .3 Outros Algoritmos . . . . . . . . . . . . . . . . . 39 
5.2 Funções básica de álgebra linear $($ BLAS $) \ldots \ldots \ldots \ldots$. . . . . . . . . . . . 41

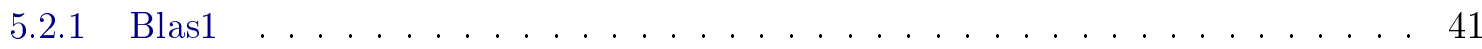

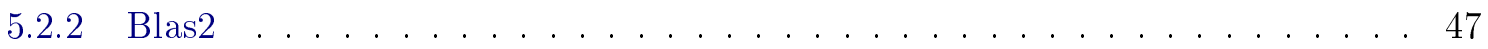

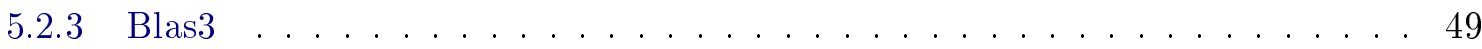

6 Wrapper e estrutura de pastas da Klein $\quad 53$

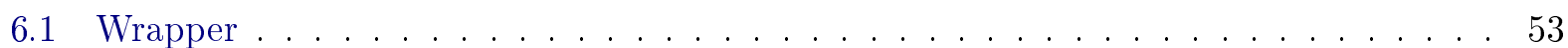

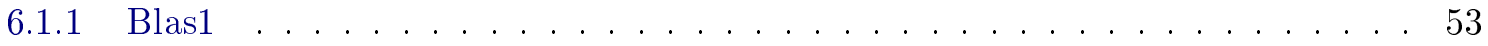

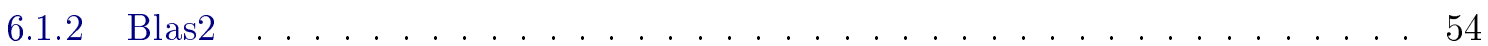

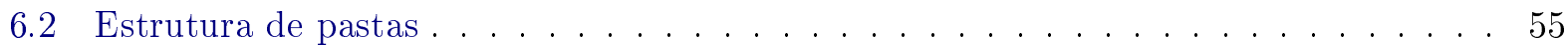

7 Teste de Software $\quad 57$

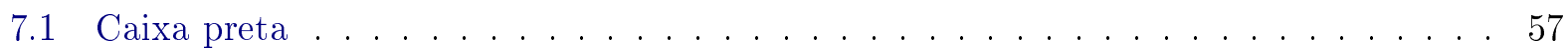

7.1.1 Particionamento em classes de equivalência . . . . . . . . . . . . . 57

7.1 .2 Análise de valor limite . . . . . . . . . . . . . . . . . . . 58

7.2 Caixa Branca . . . . . . . . . . . . . . . . . . . . . 58

7.2 .1 Cobertura por Decisão . . . . . . . . . . . . . . . . . . 58

7.2 .2 Cobertura por Condição . . . . . . . . . . . . . . . . . 59

7.2 .3 Cobertura por Decisão e Condição ～. . . . . . . . . . . . . . . . . . . 59

7.2 .4 Cobertura por Múltiplas Condições . . . . . . . . . . . . . . . . . . . . 59

7.3 Etapas de teste . . . . . . . . . . . . . . . . . 60

7.3 .1 Teste de Unidade . . . . . . . . . . . . . . . . . . . . 60

7.3 .2 Teste de Integração . . . . . . . . . . . . . . . . . . . . . . . . 60

7.3 .3 Teste de Sistema . . . . . . . . . . . . . . . . . . . 60

7.3 .4 Teste de Aceitação . . . . . . . . . . . . . . . . . . . . . . . 61

8 Testes de desempenho $\quad 63$

8.1 Soma de vetores . . . . . . . . . . . . . . . . . . . . . 63

8.2 Soma de vetor vezes escalar . . . . . . . . . . . . . . . . . . 72

8.3 Soma e subtração de vetor vezes escalar . . . . . . . . . . . . . . . . . 80

8.4 Soma de matrizes . . . . . . . . . . . . . . . . . . . . 88

8.5 Soma de matriz vezes escalar . . . . . . . . . . . . . . . . . . 93

8.6 Soma e subtração de matriz vezes escalar . . . . . . . . . . . . . . . . . . . . . 99

8.7 Multiplicação de matrizes . . . . . . . . . . . . . . . . . . . . . . 104

9 Testes genéricos $\quad 109$

9.1 Exemplo de teste genérico na Klein . . . . . . . . . . . . . . . . . . . . 109

9.2 Gerador de testes na Klein . . . . . . . . . . . . . . . . . . . . . . 112

10 Conclusões $\quad 121$

10.1 Sugestões para Pesquisas Futuras ． . . . . . . . . . . . . . . . . 121

Referências Bibliográficas $\quad 123$

$\begin{array}{ll}\text { Índice Remissivo } & 124\end{array}$ 


\section{Lista de Tabelas}

8.1 Atlas / Klein - Soma de vetores $(10 \leq n \leq 90) \ldots \ldots \ldots$. . . . . . . 65

8.2 Atlas / Klein - Soma de vetores $(100 \leq n \leq 900) \ldots \ldots \ldots 6$

8.3 Atlas / Klein - Soma de vetores $(1000 \leq \mathrm{n} \leq 9000) \ldots \ldots 6$

8.4 MKL / Klein - Soma de vetores $(10 \leq \mathrm{n} \leq 90) \ldots \ldots \ldots \ldots$. . . . . . . 67

8.5 MKL / Klein - Soma de vetores $(100 \leq \mathrm{n} \leq 900) \ldots \ldots \ldots$

8.6 MKL / Klein - Soma de vetores $(1000 \leq \mathrm{n} \leq 9000) \ldots \ldots \ldots$

8.7 Expression Template / Klein - Soma de vetores $(10 \leq \mathrm{n} \leq 90) \ldots$. . . . . . . . . 69

8.8 Expression Template / Klein - Soma de vetores $(100 \leq \mathrm{n} \leq 900) \ldots \ldots$

8.9 Expression Template / Klein - Soma de vetores $(1000 \leq \mathrm{n} \leq 9000) \ldots \ldots$. . . . . 70

8.10 Dummy / Klein - Soma de vetores $(10 \leq n \leq 90) \ldots \ldots \ldots \ldots \ldots$. . . . . . 71

8.11 Dummy / Klein - Soma de vetores $(100 \leq n \leq 900) \ldots \ldots \ldots$. . . . . . . . . . 72

8.12 Dummy / Klein - Soma de vetores $(1000 \leq n \leq 9000) \ldots \ldots \ldots$. . . . . . . 72

8.13 Atlas / Klein - Soma de vetor vezes escalar $(10 \leq \mathrm{n} \leq 90) \ldots \ldots$. . . . . . . . 74

8.14 Atlas / Klein - Soma de vetor vezes escalar $(100 \leq n \leq 900) \ldots \ldots \ldots$. . . . . . . 74

8.15 Atlas / Klein - Soma de vetor vezes escalar $(1000 \leq \mathrm{n} \leq 9000) \ldots \ldots$. . . . . . . 75

8.16 MKL / Klein - Soma de vetor vezes escalar $(10 \leq \mathrm{n} \leq 90) \ldots \ldots \ldots$

8.17 MKL / Klein - Soma de vetor vezes escalar $(100 \leq \mathrm{n} \leq 900) \ldots \ldots$. . . . . . . . 76

8.18 MKL / Klein - Soma de vetor vezes escalar $(1000 \leq \mathrm{n} \leq 9000) \ldots \ldots$

8.19 Expression Template/ Klein - Soma de vetor vezes escalar $(10 \leq \mathrm{n} \leq 90) \ldots$. . . . 77

8.20 Expression Template / Klein - Soma de vetor vezes escalar $(100 \leq \mathrm{n} \leq 900) \ldots$. . 78

8.21 Expression Template/ Klein - Soma de vetor vezes escalar $(1000 \leq \mathrm{n} \leq 9000)$. . . 78

8.22 Dummy / Klein - Soma de vetor vezes escalar $(10 \leq \mathrm{n} \leq 90) \quad \ldots \ldots \ldots$. . . . . 79

8.23 Dummy / Klein - Soma de vetor vezes escalar $(100 \leq \mathrm{n} \leq 900) \ldots \ldots$. . . . . . . 80

8.24 Dummy / Klein - Soma de vetor vezes escalar $(1000 \leq \mathrm{n} \leq 9000) \ldots \ldots$. . . . . . 80

8.25 Atlas / Klein - Soma e subtração de vetor vezes escalar $(10 \leq \mathrm{n} \leq 90) \ldots$. . . . . 82

8.26 Atlas / Klein - Soma e subtração de vetor vezes escalar $(100 \leq \mathrm{n} \leq 900) \ldots$. . . . 83

8.27 Atlas / Klein - Soma e subtração de vetor vezes escalar $(1000 \leq \mathrm{n} \leq 9000)$. . . . . 83

8.28 MKL / Klein - Soma e subtração de vetor vezes escalar $(10 \leq \mathrm{n} \leq 90) \ldots$. . . . . 84

8.29 MKL / Klein - Soma e subtração de vetor vezes escalar $(100 \leq \mathrm{n} \leq 900) \ldots$. . . . . 85

8.30 MKL / Klein - Soma e subtração de vetor vezes escalar $(1000 \leq \mathrm{n} \leq 9000)$. . . . . 85

8.31 Expression Template/ Klein - Soma e subtração de vetor vezes escalar $(10 \leq \mathrm{n} \leq 90) 86$

8.32 Expression Template / Klein - Soma e subtração de vetor vezes escalar (100 $\leq \mathrm{n} \leq$ $900) \ldots \ldots \ldots \ldots \ldots \ldots$

8.33 Expression Template / Klein - Soma e subtração de vetor vezes escalar (1000 $\leq \mathrm{n} \leq$

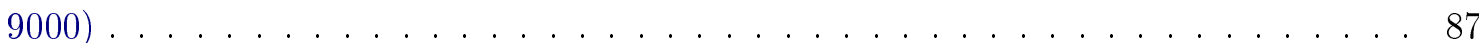


8.34 Dummy / Klein - Soma e subtração de vetor vezes escalar $(10 \leq \mathrm{n} \leq 90) \ldots$. . . . 87

8.35 Dummy / Klein - Soma e subtração de vetor vezes escalar $(100 \leq \mathrm{n} \leq 900)$. . . . 88

8.36 Dummy / Klein - Soma e subtração de vetor vezes escalar $(1000 \leq \mathrm{n} \leq 9000)$. . . . 88

8.37 MKL / Klein - Soma de matrizes $(10 \leq \mathrm{n} \leq 90) \ldots \ldots \ldots$. . . . . . . . 90

8.38 MKL / Klein - Soma de matrizes $(100 \leq n \leq 900) \ldots \ldots \ldots \ldots$. . . . . . 91

8.39 Expression Template / Klein - Soma de matrizes $(10 \leq \mathrm{n} \leq 90) \ldots \ldots$. . . . . . 91

8.40 Expression Template/ Klein - Soma de matrizes $(100 \leq \mathrm{n} \leq 900) \ldots \ldots$. . . . . . 92

8.41 Dummy / Klein - Soma de matrizes $(10 \leq n \leq 90) \ldots \ldots \ldots$. . . . . . . 93

8.42 Dummy / Klein - Soma de matrizes $(100 \leq n \leq 900) \ldots \ldots \ldots$. . . . . . . . 93

8.43 MKL / Klein - Soma de matriz vezes escalar $(10 \leq \mathrm{n} \leq 90) \ldots \ldots \ldots$. . . . . 95

8.44 MKL / Klein - Soma de matriz vezes escalar $(100 \leq \mathrm{n} \leq 900) \ldots \ldots$. . . . . . . 96

8.45 Expression Template / Klein - Soma de matriz vezes escalar $(10 \leq \mathrm{n} \leq 90) \quad \ldots$. . 97

8.46 Expression Template/ Klein - Soma de matriz vezes escalar $(100 \leq \mathrm{n} \leq 900)$. . . 97

8.47 Dummy / Klein - Soma de matriz vezes escalar $(10 \leq \mathrm{n} \leq 90) \ldots \ldots$. . . . . . 98

8.48 Dummy / Klein - Soma de matriz vezes escalar $(100 \leq \mathrm{n} \leq 900) \ldots \ldots$

8.49 MKL / Klein - Soma e subtração de matriz vezes escalar $(10 \leq \mathrm{n} \leq 90) \ldots$. . . . . 101

8.50 MKL / Klein - Soma e subtração de matriz vezes escalar $(100 \leq \mathrm{n} \leq 900)$. . . . . 102

8.51 Expression Template / Klein - Soma e subtração de matriz vezes escalar $(10 \leq \mathrm{n} \leq 90) 102$

8.52 Expression Template / Klein - Soma e subtração de matriz vezes escalar $(100 \leq \mathrm{n} \leq$

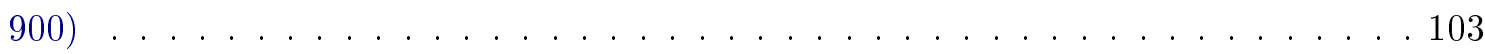

8.53 Dummy / Klein - Soma e subtração de matriz vezes escalar $(10 \leq \mathrm{n} \leq 90) \ldots 104$

8.54 Dummy / Klein - Soma e subtração de matriz vezes escalar $(100 \leq \mathrm{n} \leq 900)$. . . . 104

8.55 Atlas / Klein - Multiplicação de matriz $(10 \leq \mathrm{n} \leq 90) \ldots \ldots$. . . . . . . . . . 106

8.56 Atlas / Klein - Multiplicação de matriz $(100 \leq \mathrm{n} \leq 900) \ldots \ldots$. . . . . . . . 106

8.57 MKL / Klein - Multiplicação de matriz $(10 \leq \mathrm{n} \leq 90) \ldots \ldots$. . . . . . . . . . . 107

8.58 MKL / Klein - Multiplicação de matriz $(100 \leq \mathrm{n} \leq 900) \ldots \ldots$. . . . . . . . 107 


\section{Capítulo 1}

\section{Introdução}

A Klein é uma biblioteca genérica para álgebra linear. Ela permite manipular matrizes e vetores em $\mathrm{C}++$ de forma intuitiva, de modo similar ao Matlab. Com ela podemos, por exemplo, implementar um passo do Método de Newton para a função $f$, através da expressão

$$
x=x-i n v(j a c(x)) * f(x),
$$

onde $\mathrm{x}$ é vetor, jac a Jacobiana e $i n v$ a inversa.

A biblioteca foi construída utilizando o paradigma de programação genérica, cujo propósito é a construção de algoritmos e estruturas na forma mais genérica. Com isso, é possível a instanciação de um objeto para diferentes tipos numéricos. Em $\mathrm{C}++$ isso é feito através de um recurso da linguagem chamado templates, que permite polimorfismo em tempo de compilação.

Com as chamadas de funções definidas em tempo de compilação, esperávamos alguns ganhos de desempenho. Portanto, para verificarmos a eficiência da biblioteca, comparamos o desempenho da Klein com bibliotecas otimizadas para álgebra linear, e com diferentes estratégias de avaliação de expressões. Os resultados desses testes definiram algumas estratégias para implementação de melhorias.

Além disso, nesse trabalho foram construídos wrappers para algumas funções das bibliotecas otimizadas MKL (Intel Math Kernel Library)[9] e ATLAS (Automatically Tuned Linear Algebra Software)[3]. Dessa forma, para alguns tipos numéricos e algumas funções essas bibliotecas podem ser invocadas no lugar da implementação genérica da Klein. Mostramos também como podemos criar wrappers para outras bibliotecas.

Por se tratar de uma biblioteca genérica, era necessário que os testes também fossem genéricos. Nesse trabalho discutimos como esses testes funcionam na Klein e como a biblioteca consegue, em tempo de compilação, gerar casos de teste através da combinação de fábricas.

\subsection{Objetivos}

Neste trabalho, estudamos, testamos e documentamos a biblioteca Klein. Esta biblioteca manipula matrizes e vetores de forma simplificada, mas precisa ter uma ampla cobertura de testes para que possa ser disponibilizada para a comunidade. Para guiar e definir estratégias de desenvolvimento de algoritmos e como a ferramenta vai se comportar em pontos específicos, foram necessários testes de desempenho. Portanto, os objetivos deste trabalho são garantir o correto funcionamento da ferramenta Klein, através de testes genéricos, e auxiliar o desenvolvimento, comparando seu desempenho com outras estratégias e bibliotecas conhecidas.

\subsection{Organização do Trabalho}

Além dessa introdução, o trabalho contém 9 capítulos. No Capítulo 2 explicamos como a linguagem $\mathrm{C}++$ suporta a programação genérica através de templates. No Capítulo 3 descrevemos a arquitetura e as estratégias utilizadas na biblioteca Klein. O capítulo 4 contém os objetos da Klein e é seguido pelo capítulo 5 com os algoritmos e funções da Klein. No capítulo 6 explicamos como criar um wrapper para bibliotecas otimizadas e apresentamos a estrutura de pastas da Klein. No Capítulo 7 apresentamos as técnicas e etapas de teste de software. No capítulo 8 apresentamos os testes de desempenho efetuados e o que ganhamos com eles. No capítulo 9 explicamos como 
funcionam os testes genéricos criados para o teste da Klein. E finalizamos com o capítulo 10, onde apresentamos as conclusões do trabalho. 


\section{Capítulo 2}

\section{Programação Genérica}

Programação genérica [5] é uma abordagem para projetar e organizar software que busca encontrar a forma mais genérica de um algoritmo em conjunto com uma implementação eficiente. Nos programas genéricos, os objetos e algoritmos são criados em uma estrutura lógica independente do tipo de dado a ser manipulado. Isto possibilita que um algoritmo escrito uma única vez possa ser instanciado para vários tipos de dados.

Esta abordagem consiste de três pontos principais: abstração, parametrização e instanciação. A abstração é a escrita de um algoritmo pensando apenas no seu propósito, sem pensar na estrutura que ele irá manipular. A parametrização é a possibilidade de definir o tipo a ser manipulado no momento que estamos programando. E a instanciação ocorre quando o compilador identifica o tipo e cria o código com a estrutura desejada.

Com a utilização deste paradigma reduzimos a quantidade de código duplicado. Uma função ou classe, criada para um propósito específico, é escrita apenas uma vez e reutilizada para vários tipos de dados. Como o mesmo algoritmo não precisa ser escrito várias vezes, reduzimos a quantidade de erros decorrente da reescrita e o tempo de desenvolvimento acaba sendo reduzido, já que não há criação e teste de novas linhas de código.

O exemplo clássico de biblioteca genérica é a STL (Standard Template Library), que complementa as versões modernas da linguagem $\mathrm{C}++$. Os principais componentes desta biblioteca são contêiners, iteradores, algoritmos e objetos de função. Contêiners são estruturas de dados para armazenamento de massa de dados, são exemplos: listas, filas, vetores e pilhas. Iteradores são como ponteiros, pois apontam para os elementos de um contenedor e são utilizados para acessar seus valores. Algoritmos são funções para propósitos específicos como ordenação e busca. Objetos de função são objetos que comportam-se como funções e permitem especializar o código para manipulação de dados conforme uma aplicação específica. Por exemplo, as funções objetivo, os Jacobianos e as Hessianas de problemas de otimização podem ser representadas por objeto de função.

\subsection{Templates em $\mathrm{C}++$}

Templates são recursos da linguagem de programação $\mathrm{C}++[12]$ que permitem a programação genérica. Eles geram código, em tempo de compilação, para o tipo sendo instanciado. Abaixo veremos conceitos básicos sobre templates em $\mathrm{C}++$ e exemplos de sua utilização.

\subsubsection{Funções Template}

Funções Template lidam com argumentos de tipos genéricos. Isso nos permite criar uma função que pode se adaptar a mais de um tipo de dado ou classe, sem repetição de código.

Em $\mathrm{C}++$ isso é feito através da declaração de um tipo com o seguinte comando:

1 template <typename identifier> declaracao_funcao;

Os tipos declarados por estes comandos são utilizados pelas funções como se fossem um tipo regular. Por exemplo, para criar uma função template para retornar o maior de dois objetos podemos usar:

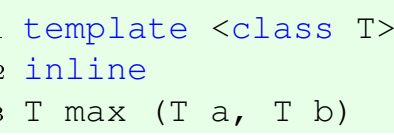




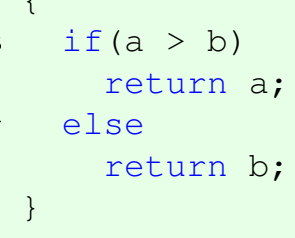

onde T representa um tipo que ainda não foi especificado. Quando essa função é chamada com um tipo regular, ela retorna o maior entre os dois elementos. Para comparar dois inteiros podemos usar essa função conforme segue:

1 int $x, y$;

$2 \max (x, y)$;

Quando o compilador encontra a chamada para uma função template, ele efetua a troca de todas as ocorrências de $\mathrm{T}$ pelo tipo passado como parâmetro na função. Este processo é realizado automaticamente pelo compilador e é invisível para o programador.

Abaixo o exemplo de um programa:

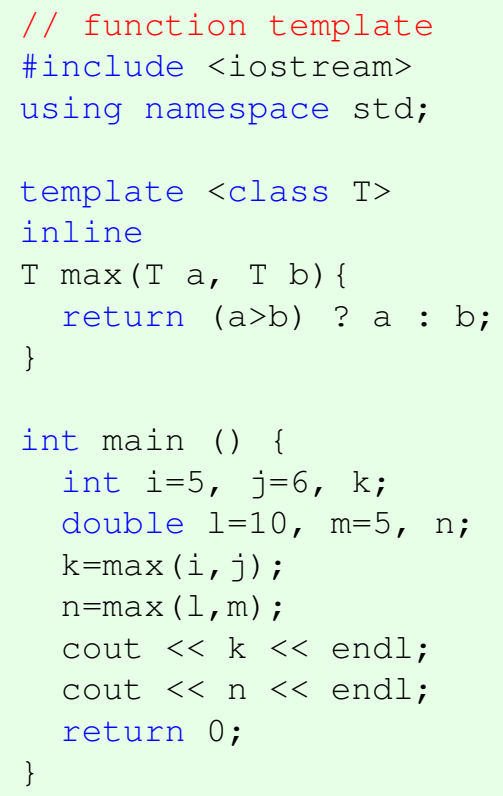

No exemplo, a função template max é chamada duas vezes. Na primeira vez é chamada com o argumento de tipo int e na segunda vez com o argumento de tipo double. O compilador entende e chama a versão apropriada da função para cada instanciação.

No código acima, não seria possível comparar tipos diferentes. Para isto, a função deveria ser declarada da seguinte forma:

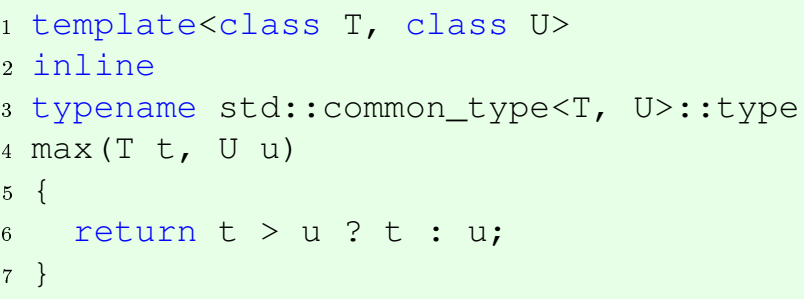

O retorno desta função é definido pela classe trait std::common_type $<T, U>$ (veremos o que são classes traits na seção (3.3)). Esse exemplo ilustra o uso de meta programação em $\mathrm{C}++$. Ou seja, ele mostra que podemos implementar (meta) funções que associam tipos a tipos. No exemplo abaixo, std::common_type <int, double $>$ ::type associa o tipo double ao par de tipos int e double, basicamente porque este é o tipo em que ambos podem ser convertidos implicitamente sem perda de informação. 
double $d=7.8$;

$3 \mathrm{~d}=\max (i, d)$;

\subsubsection{Classes Template}

Classes template são classes definidas a partir de tipos genéricos de dados. Estas classes recebem os parâmetros para definição do tipo da mesma forma que as funções templates:

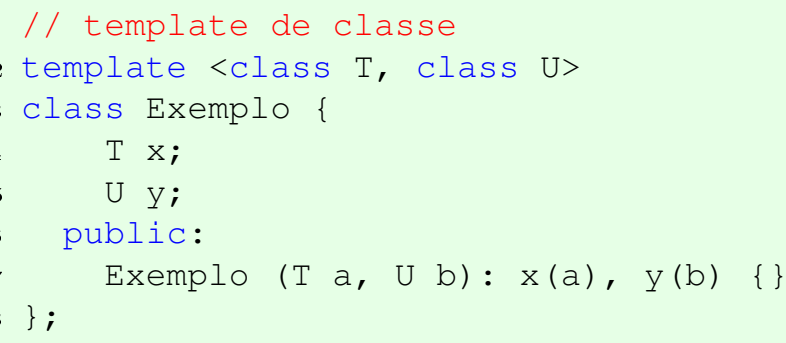

A classe que acabamos de definir armazena dois elementos de quaisquer tipos de dados $T$ e $U$. Por exemplo, se queremos declarar um objeto desta classe para armazenar os valores inteiros $115 \mathrm{e}$ 36 escrevemos:

1 Exemplo<int, int> objeto $(115,36)$;

esta mesma classe poderia ser usada para armazenar valores do tipo double e int:

1 Exemplo<double, int> objeto (3.432, 2);

\subsubsection{Especialização de Templates}

Se quisermos definir uma implementação específica para um tipo de dado em um template, podemos declarar uma especialização daquele template. Por exemplo, vamos supor que temos uma simples classe chamada Recipiente que armazena um elemento de qualquer tipo e possui uma função chamada incrementa, que incrementa seu valor. Porém quando esta classe armazena o tipo char, queremos que ela se comporte de forma diferente. Neste caso, necessitamos de uma especialização deste template:

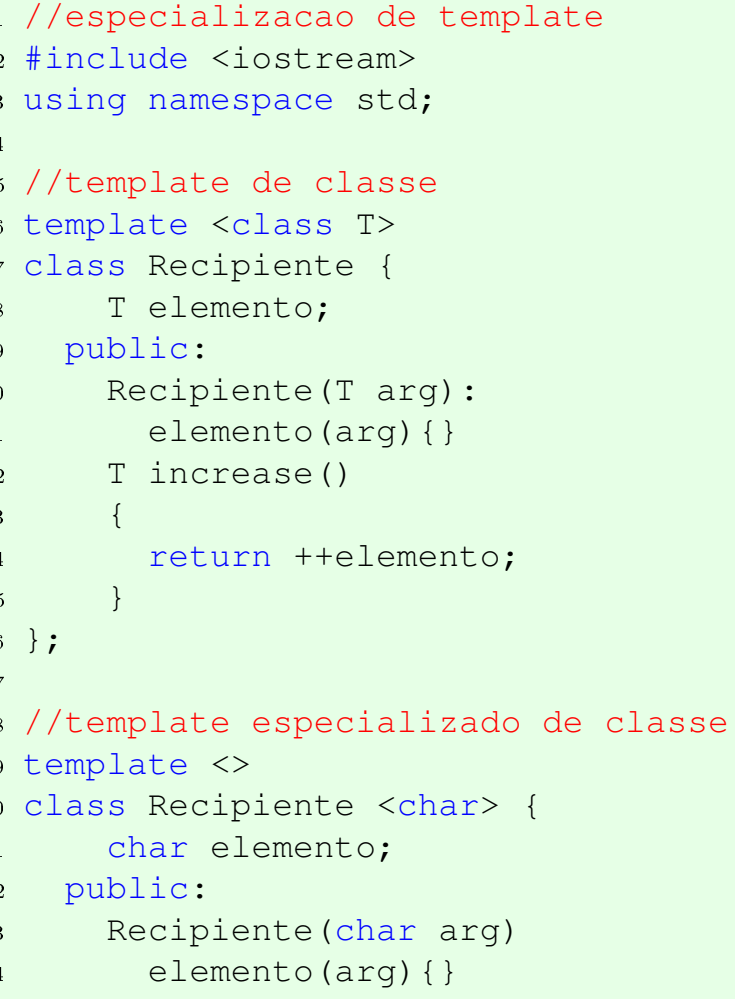




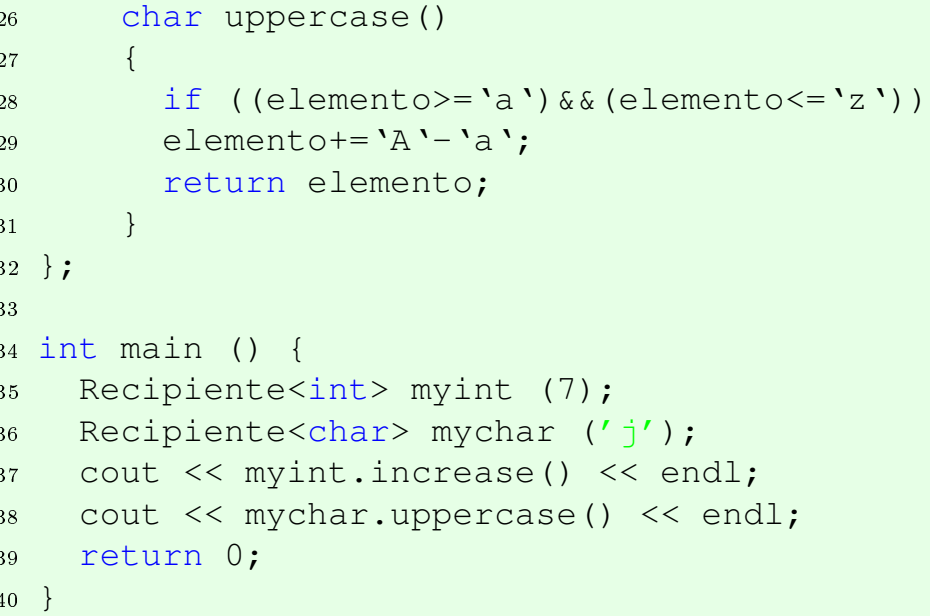

Note que o parâmetro de tipo do dado não é passado na especialização do template da classe para deixar explicito que se trata de uma especialização. Todavia, mais importante que este prefixo, é o parâmetro de especialização $<$ char $>$ depois do nome da classe. Este último identifica o tipo da classe a qual se está especializando.

\subsection{Tipos genéricos de números utilizando templates}

Neste tópico descreveremos duas funções $\mathrm{C}++$ que, através do uso de template, permitem a utilização de tipos genéricos de números. A primeira função chama-se abs. Ela recebe um intervalo (a Klein disponibiliza uma classe Interval) como parâmetro e retorna o módulo deste intervalo.

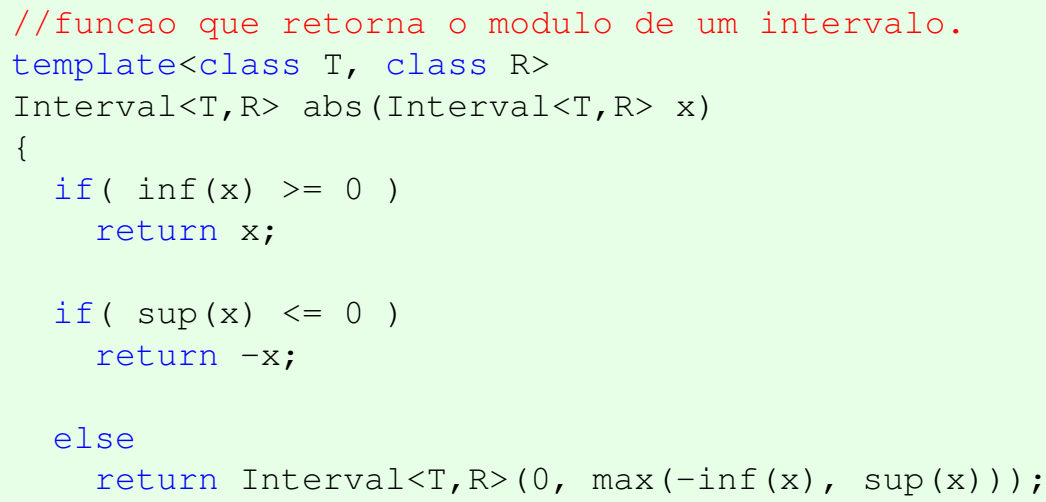

Podemos chamá-la desta forma:

1 Interval<double> $\mathrm{x}(-3,1)$;

2 Interval<double $>\mathrm{y}=\operatorname{abs}(\mathrm{x})$;

A segunda função consiste do cálculo da expressão $x+=x^{*} y-z^{*} y$. Ela recebe como parâmetro os valores de $\mathrm{x}, \mathrm{y}$ e z, e retorna o resultado do cálculo.

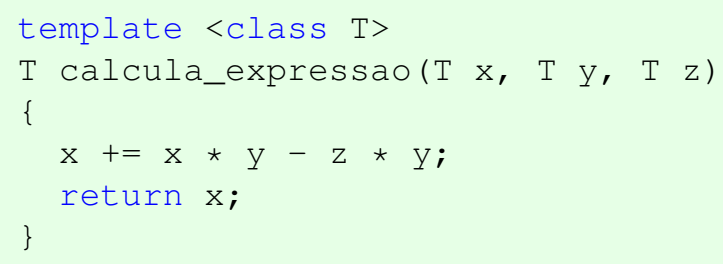

Abaixo serão apresentadas três chamadas para a função, uma para cada tipo de número. A primeira invoca o cálculo da função utilizando o tipo float:

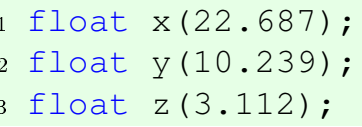


A segunda é a invocação para o tipo double:

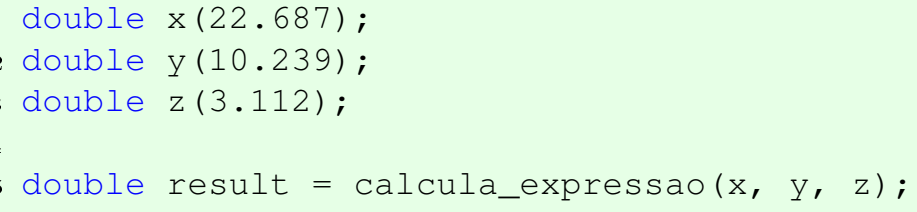

A terceira chamada é para o tipo Interval, implementado na Klein:

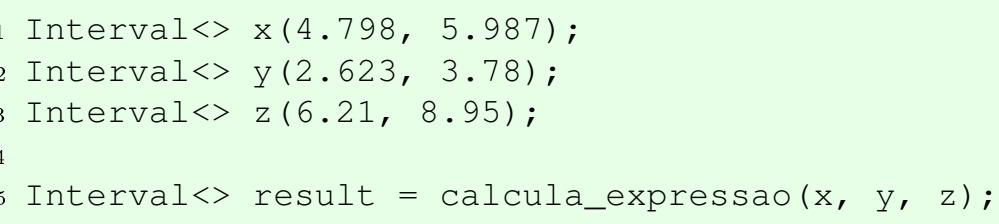

E a quarta chamada é para o cálculo através da biblioteca MPFR(Multiple Precision Floating Point) [8]. Essa biblioteca lida com números de ponto flutuante de precisão múltipla, cuja precisão pode ser definida em tempo de execução. Um problema desta biblioteca é não possuir os operadores aritméticos como os tipos primitivos da linguagem. Portanto, para invocar a função calcula_expressao para este tipo é necessário criar uma interface entre ela e a chamada da biblioteca, contendo no mínimo a sobrecarga dos operadores $+=,-\mathrm{e}^{*}$. Para isto, criamos a classe Real, descrita abaixo:

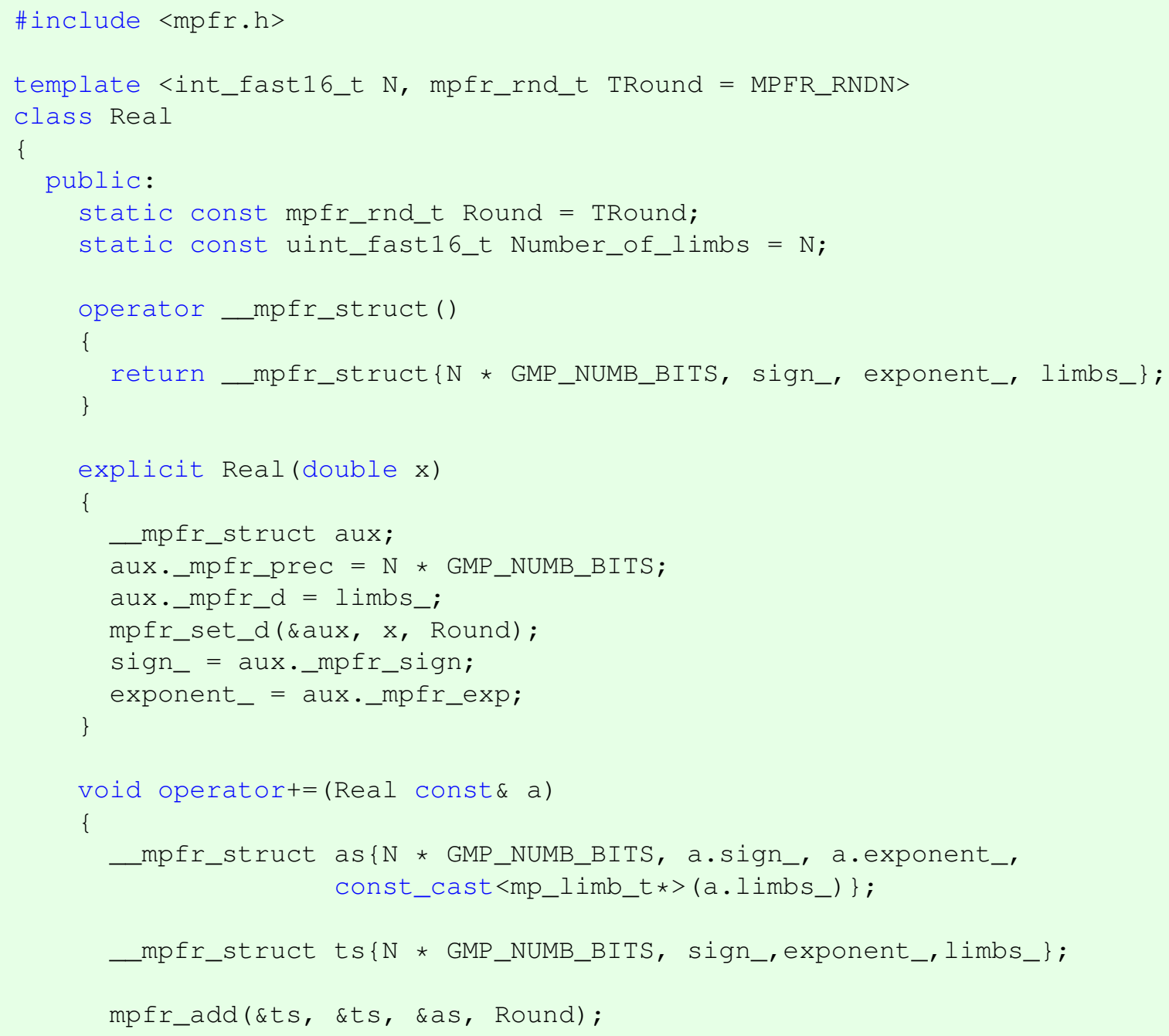




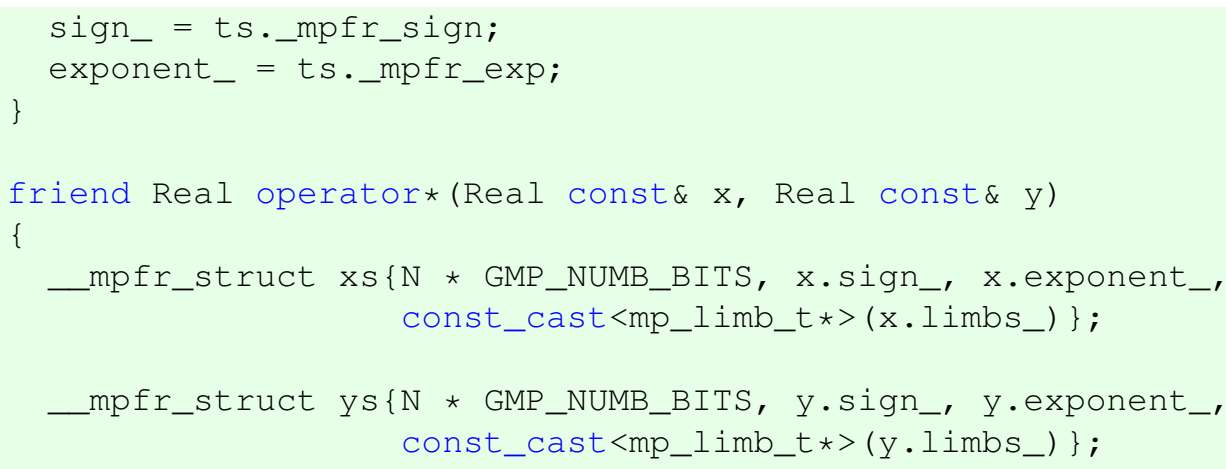


O código foi utilizado para reescrever a forma como a biblioteca efetua as operações aritméticas, fazendo com que sua invocação seja da mesma forma que para os tipos primitivos da linguagem.

Note que a interface foi construída como uma classe template para permitir que a precisão seja especificada no momento da instanciação. Nesse caso, a precisão será definida em tempo de compilação. Para uma precisão de 5x64=320 bits, chamaríamos a função da seguinte forma:

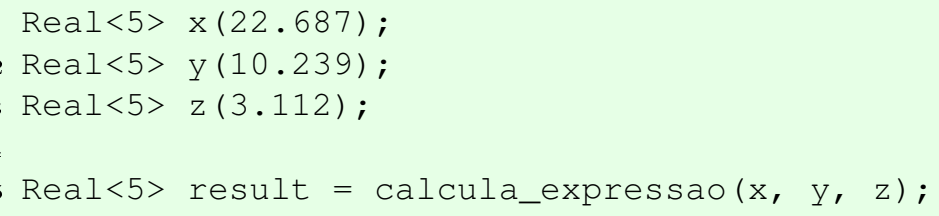

Se precisássemos de uma precisão maior, bastaríamos alterar o parâmetro template:

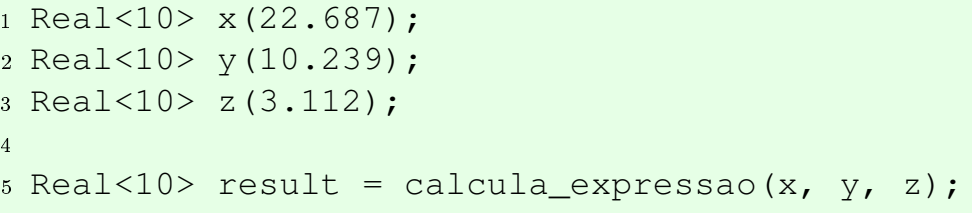

Em um primeiro momento podemos pensar que houve um grande trabalho para o reaproveitamento de apenas uma função. Porém este trabalho deve ser feito uma única vez e a medida que apareçam novas funções haverá menos necessidade de alterações no código da classe Real. Sem contar que poderemos utilizar esta biblioteca em qualquer algoritmo genérico. 



\section{Capítulo 3}

\section{Arquitetura e estratégias da Klein}

A Klein é organizada em dois níveis: a parte abstrata, com matrizes, vetores e operadores lineares e a parte básica (BLAS), que implementa as operações, ou delega essas operações para bibliotecas otimizadas, como a $M K L$ e a ATLAS. Ou seja, para alterar a forma como a soma é feita, não há necessidade de alterar a forma como a soma é invocada. Isto significa que podemos utilizar bibliotecas otimizadas para os casos que julgarmos necessário apenas incluindo os wrappers das bibliotecas.

O usuário interage com a parte abstrata, e a parte básica pode ser substituída sem a necessidade de alterar o código fonte do usuário. Por exemplo, o usuário poderia usar seu código com a $M K L$ em algumas máquinas e com a $A T L A S$ em outras. Porém devido à restrição do $\mathrm{C}++$, será necessário compilar uma versão do código para cada BLAS. A idéia é que a parte abstrata nunca mude, mas que otimizações sejam implementadas na parte básica.

Abaixo uma forma macro de enxergar a arquitetura da Klein:

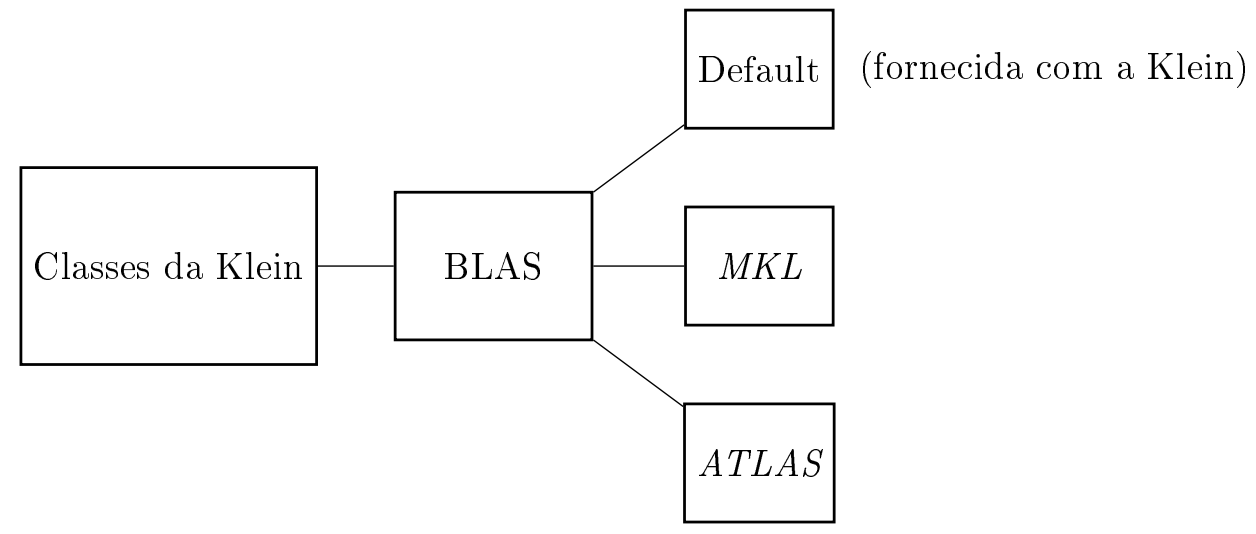

O primeiro bloco, Classes da Klein, é onde se encontram as estruturas de dados da Klein. Logo depois, vem o bloco $B L A S$ que pode ser composto pelas outras três partes. Os blocos, $M K L$ e $A T L A S$, são exemplos de bibliotecas otimizadas que podem ser chamadas para processar as operações. O bloco Default é fornecido pela Klein, é correto, mas pode ser ineficiente. Cabe ao usuário escolher o que é mais vantajoso para ele. A criação dos wrappers pode ser feita incluindo o código dos wrappers no arquivo blas1_opt.h existente na pasta: klein/blas/optimized.

Por se tratar de uma biblioteca genérica os parâmetros templates são resolvidos em tempo de compilação, não havendo perda de tempo com chamadas de funções. Além disso, como todas as funções são criadas inline, quando compilamos o código utilizando o parâmetro de otimização -O3 ele copia o código das funções para o ponto de chamada e otimiza o código como um todo. Por exemplo, ao criarmos um wrapper para a $M K L$, a camada da Klein responsável pelas chamadas das funções será resolvida em tempo de compilação, e em tempo de execução só haverá a chamada para a função da $M K L$.

Devido uma limitação do $\mathrm{C}++$, é necessário compilar o código da biblioteca otimizada junto com o código da Klein. Isso fará com que o código seja criado para o tipo sendo instanciado, além de permitir a otimização da chamada das funções da biblioteca. 


\subsection{Estratégias para avaliar expressões}

Em geral, expressões aritméticas podem ser avaliadas de dois modos. A avaliação eager, avalia a expressão imediatamente, e a avaliação lazy, que adia a avaliação o máximo que puder. Abaixo mais detalhes sobre essas estratégias.

\section{Estratégia eager (impaciente)}

A estratégia eager (impaciente) avalia uma expressão tão logo seja possível. Esta é a estratégia de avaliação utilizada pelas linguagens de programação mais tradicionais. Ela elimina a necessidade de controlar e programar a avaliação das expressões, além de permitir que o programador dite a ordem de execução facilmente. Porém é também o programador quem deverá organizar o código da maneira mais otimizada.

Para o exemplo abaixo,

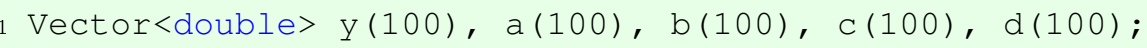

a avaliação eager procederia desse modo:

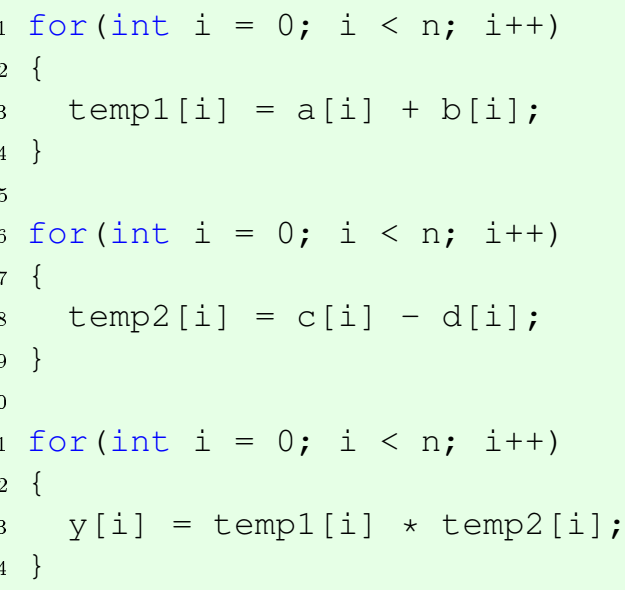

\section{Estratégia lazy (preguiçosa)}

A estratégia lazy (preguiçosa) adia a avaliação da expressão até que o valor seja necessário. Essa estratégia pode evitar o uso de memória temporária e permite que a avaliação seja feita de forma otimizada. Por exemplo, a expressão abaixo poderia ser avaliada da seguinte forma:

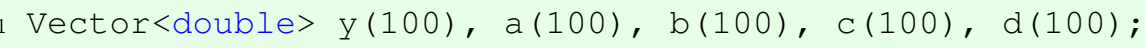

Uma forma de aproveitarmos o comportamento Lazy para avaliarmos esta expressão, seria efetuarmos a soma entre os vetores $a$ e $b$, armazenarmos o valor em $y$ e, posteriormente, efetuar a subtração entre $c$ e $d$ e multiplicar de forma inplace os elementos de $y$ (quando efetuamos uma operação aritmética junto com uma atribuição em uma única instrução, isso é chamado de operação inplace). Conforme segue:

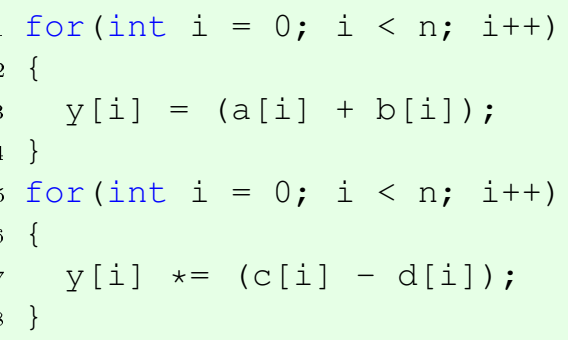

Outra forma de avaliar esta expressão aproveitando o comportamento Lazy do código é utilizando a técnica de expression templates. Esta técnica avalia elemento a elemento da expressão, e ao fazer 
isto pode tornar o código mais eficiente. Para o código acima, a avaliação utilizando esta técnica ocorreria desta forma:

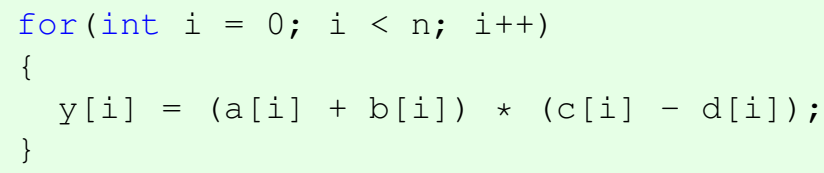

Comparamos os tempos de execução dos três algoritmos citados acima em uma máquina com Intel@ Core $^{\mathrm{TM}} \mathrm{i} 7-3610 \mathrm{QM}$ CPU @ 2.30GHz x 8, 8GB de RAM, sistema operacional Ubuntu 13.10 e utilizando o compilador gcc 4.8.1.

\begin{tabular}{|c|c|c|c|c|}
\hline Tempos relativos & \multicolumn{4}{|c|}{ Número de elementos } \\
\hline Funções & 1000 & 10000 & 100000 & 1000000 \\
\hline eager_calc & 1 & 1 & 1 & 1 \\
lazy_memory_calc & 0,75 & 0,35 & 0,26 & 0,47 \\
lazy_expression_calc & 0,51 & 0,27 & 0,23 & 0,37 \\
\hline
\end{tabular}

Chamamos de eager_calc o código $\mathrm{C}++$ que utiliza a estratégia eager. As estratégias lazy são dividas nos códigos lazy_memory_calc, que evita a criação de vetores temporários, e lazy_expression_calc, que usa a técnica de expression template. Para melhor compararmos os tempos entre os algoritmos, normalizamos o tempo do algoritmo eager_calc para 1 e os tempos dos outros dois são relativos a este primeiro. Ou seja, os valores na tabela indicam o percentual de tempo que os algoritmos levam com relação ao tempo do primeiro.

Nesse caso, fica evidente a vantagem de utilizarmos a estratégia lazy, em especial com a técnica expression template. Essa é a grande vantagem desta estratégia, termos todas as informações a respeito da expressão antes de iniciar o cálculo, o que nos permite avaliar a expressão da forma mais apropriada.

\subsubsection{Estratégia Lazy na Klein}

A biblioteca Klein utiliza estratégias Lazy para avaliar expressões. Porém não chega à granularidade da técnica expression template em todos os casos. O que ocorre é evitar consumo de memória temporária e otimização de algumas expressões. Como é o caso da expressão entre os escalares $y=a x+y$, que pode ser calculada em um único passo pelo processador utilizando a instrução FMA [7].

Apesar de a Klein não possuir todas as otimizações possíveis de expressões, nada impede que isto seja acrescentado futuramente de forma transparente para o usuário. Ou seja, ao receber uma nova versão da Klein com uma série de otimizações de expressões o usuário não precisará se preocupar com seu código antigo, pois ele continuará funcionando e passará a rodar em cima destas otimizações.

A Klein só avalia uma expressão no momento em que há uma atribuição a uma variável. Isso significa que ela irá montar a árvore referente aquela expressão e só depois definirá como a atribuição será feita. Para a seguinte função $h=x+y+z$, onde $h, x, y$ e $z$ são vetores, teríamos a árvore abaixo:

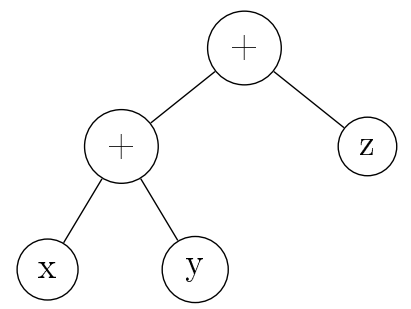

que seria calculada pela Klein somando $x+y$ e armazenando em $h$ e, posteriormente, somando $z$ de forma inplace em $h$.

Para que seja possível este comportamento lazy foram implementadas sobrecargas dos operadores aritméticos. Elas são específicas para cada momento da avaliação. O exemplo acima seria tratado 
pela Klein em três passos. O primeiro é a identificação da soma entre os vetores $x$ e $y$. Neste momento, é criado um objeto de uma classe lazy, chamada vector_plus_vector. Este objeto contém um apontador para os elementos do vetor $x$, um apontador para os elementos do vetor $y$ e o número de elementos desses vetores. O segundo passo é a identificação da soma entre o objeto lazy vector_plus_vector e o vetor $z$. Neste momento, é criado outro objeto lazy que chamaremos de lazy_plus_vector. Ele possui uma cópia do objeto vector_plus_vector, um apontador para os elementos do vetor $z$ e o número de elementos dos vetores. E o terceiro passo, que é a atribuição propriamente dita. A figura abaixo demonstra como fica a estrutura criada por estes passos:

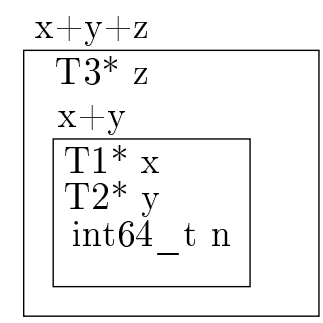

A atribuição, contudo, também não ocorre em um único passo. O primeiro passo é solicitar ao objeto lazy_plus_vector que retorne o seu valor. Neste momento, ele solicita a seus objetos vector_plus_vector e vetor $z$ que façam o mesmo. O objeto vector_plus_vector retornará a soma entre $x$ e $y$ no vetor $h$, e em seguida o vetor $z$ será somado ao vetor $h$. Esta soma entre os vetores, que ocorre somente no momento da atribuição, pode ser feita utilizando técnicas de expression templates ou simplesmente invocando bibliotecas de alta desempenho como a $M K L$.

Para a função $y=(a+b) *(c+d)$, onde $y, a, b, c$ e $d$ são matrizes, teríamos a árvore abaixo:

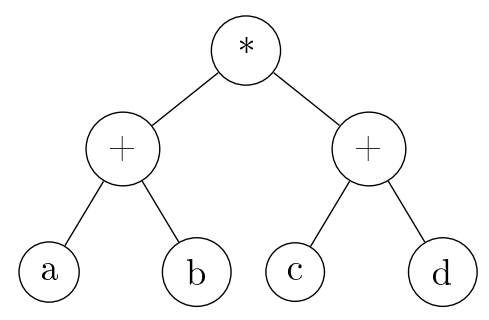

e a estrutura criada pela sobrecarga dos operadores aritméticos:

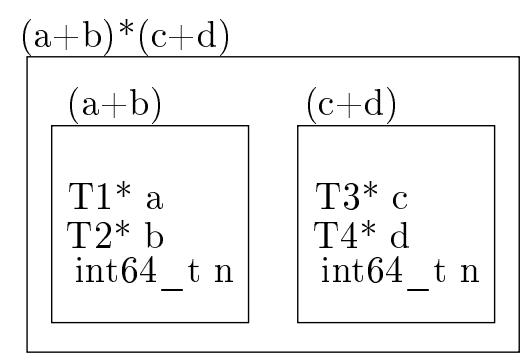

\section{$3.2 \quad$ Alias}

Quando manipulamos matrizes ou vetores devemos tomar o devido cuidado com a sobreposição de memória, pois podemos estar atribuindo um valor a uma posição que ainda será utilizada, este problema é chamado de alias. Isto é comum quando efetuamos operações aritméticas com subvetores, onde o vetor sendo manipulado é subvetor do vetor sendo atribuído. O mesmo ocorre com submatrizes. 
Tendo em mãos os vetores $\mathrm{x}$ e $\mathrm{y}$, e os subvetores $\mathrm{h}$ e $\mathrm{z}$,

$$
x=\left[\begin{array}{l}
a \\
b \\
c \\
d \\
e \\
f
\end{array}\right] \quad y=\left[\begin{array}{l}
i \\
j \\
k
\end{array}\right] \quad h=\left[\begin{array}{l}
b \\
c \\
d
\end{array}\right] \quad z=\left[\begin{array}{l}
c \\
d \\
e
\end{array}\right]
$$

onde a posição inicial de $z$ aponta para o terceiro elemento de $x$ e a posição inicial de $h$ aponta para o segundo elemento de $x$.

$$
\begin{array}{l|l}
\mathrm{x} \longrightarrow \\
\mathrm{z} \longrightarrow & \mathrm{a} \\
\mathrm{b} \\
\mathrm{c} \\
\mathrm{d} \\
\mathrm{e} \\
\mathrm{f}
\end{array} \mid
$$

Ao efetuarmos o cálculo da expressão $z=h+y$, o primeiro passo é somar $h[0]+y[0]$ e atribuir à posição $z[0]$.

$$
\begin{array}{c|c}
\mathrm{x} \longrightarrow> & \mathrm{a} \\
\mathrm{h} \longrightarrow & \mathrm{b} \\
\mathrm{b}+\mathrm{i} \\
\mathrm{d} \\
\mathrm{e} \\
\mathrm{f}
\end{array} \mid
$$

Isto fará com que a posição $h[1]$ seja modificada, pois ambos apontam para a mesma posição de memória. Sendo assim, a soma $h[1]+y[1]$ irá se comportar de maneira inesperada, visto que no lugar de somar $c+j$, será feita a soma $b+i+j$. O mesmo ocorrerá para a segunda posição dos vetores. No final da expressão o vetor resultante será

$$
\begin{array}{c|c}
\mathrm{x} \longrightarrow \\
\mathrm{z} \longrightarrow & \mathrm{b} \\
\mathbf{b}+\mathbf{i} \\
\mathbf{b}+\mathbf{i}+\mathbf{j} \\
\mathbf{b}+\mathbf{i}+\mathbf{j}+\mathbf{k} \\
\mathrm{f}
\end{array} \mid
$$

enquanto que o esperado era

$$
\begin{array}{c|c}
\mathrm{x} \longrightarrow \\
\mathrm{h} \longrightarrow \\
\mathrm{z}-> \\
\mathbf{b}+\mathbf{i} \\
\mathbf{c}+\mathbf{j} \\
\mathbf{d}+\mathbf{k} \\
\mathrm{f}
\end{array} \mid
$$

Utilizando os mesmos vetores e a mesma sobreposição de memória podemos ter casos em que não há alias. Por exemplo, se trocarmos $z$ e $h$ de posição na expressão original teríamos $h=z+y$. O primeiro passo seria somar $z[0]$ e $y[0]$ e armazenar em $h[0]$ :

$$
\begin{array}{l|c}
\mathrm{x}-> & \mathrm{a} \\
\mathrm{h}-> & \mathbf{c}+\mathbf{i} \\
\mathrm{c} \\
\mathrm{d} \\
\mathrm{e} \\
\mathrm{f}
\end{array} \mid
$$


Após o cálculo de toda a expressão verificamos que apesar da sobreposição de memória, não há problema de alias.

$$
\begin{gathered}
\mathrm{x} \longrightarrow \\
\mathrm{h}-> \\
\mathrm{z}-> \\
\mathbf{c}+\mathbf{i} \\
\mathbf{d}+\mathbf{j} \\
\mathbf{e}+\mathbf{k} \\
\mathrm{e} \\
\mathrm{f}
\end{gathered} \mid
$$

\subsubsection{Alias na Klein}

Quando codificamos a expressão $z=h+y$ citada acima sem usar a Klein, notamos que, se não tomarmos cuidado, podemos nos defrontar com problemas de alias.

$$
x=\left[\begin{array}{l}
4 \\
6 \\
9 \\
2 \\
1 \\
3
\end{array}\right] \quad y=\left[\begin{array}{l}
8 \\
7 \\
3
\end{array}\right] \quad h=\left[\begin{array}{l}
6 \\
9 \\
2
\end{array}\right] \quad z=\left[\begin{array}{l}
9 \\
2 \\
1
\end{array}\right]
$$

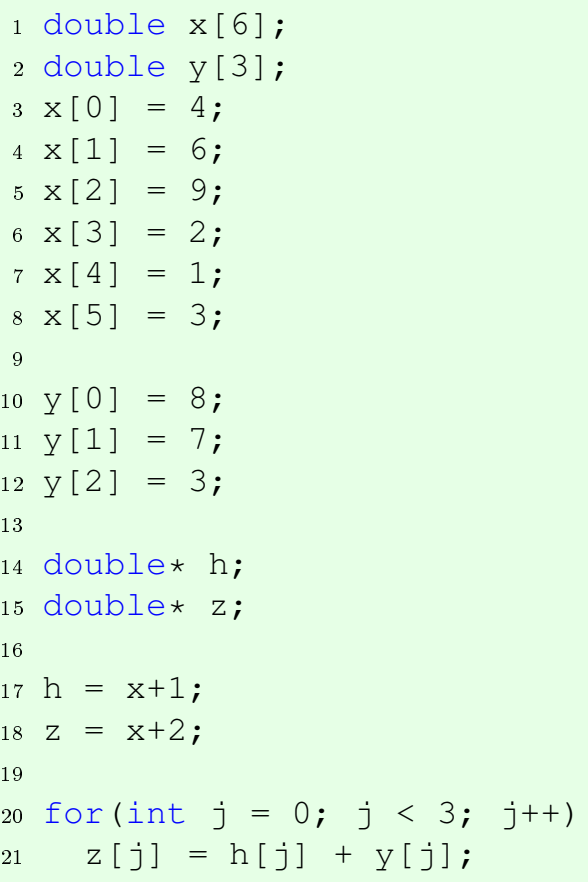

Resultado:

$$
x=\left[\begin{array}{r}
4 \\
6 \\
14 \\
21 \\
24 \\
3
\end{array}\right]
$$

A forma como efetuamos a soma fez com que o resultado fosse diferente do que esperávamos. Para evitar que isto ocorra, temos que substituir $z$ por um vetor temporário e, somente depois de ter o vetor resultante em mãos, atribuí-lo a $z$, conforme abaixo:

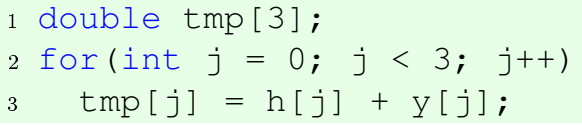


Resultado:

$$
x=\left[\begin{array}{r}
4 \\
6 \\
14 \\
16 \\
5 \\
3
\end{array}\right]
$$

Outra forma de resolver o problema de alias, neste caso, seria efetuar a soma de trás para frente direto em $z$.

1 for (int $j=2 ; j>=0 ; j--$ )

$2 \mathrm{z}[j]=h[j]+\mathrm{y}[j]$;

Resultado:

$$
x=\left[\begin{array}{r}
4 \\
6 \\
14 \\
16 \\
5 \\
3
\end{array}\right]
$$

A Klein nos poupa destas preocupações fazendo a verificação de alias antes de efetuar uma atribuição. Desta forma, este mesmo código traduzido para a Klein ficaria como descrito abaixo e não teríamos problemas com alias.

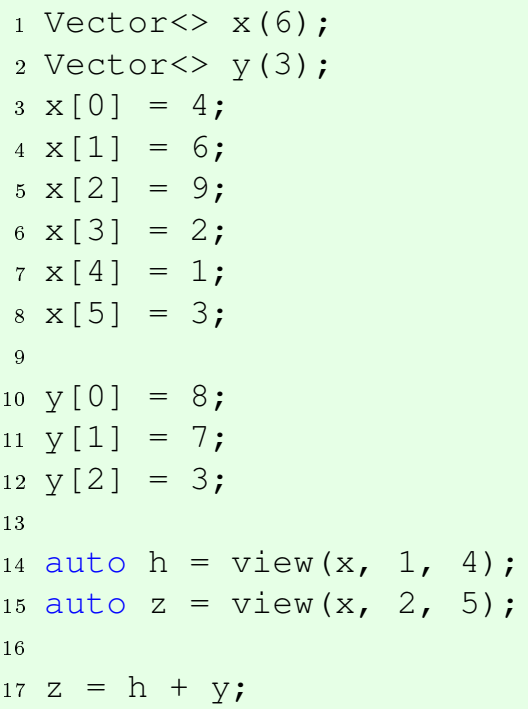

Resultado:

$$
x=\left[\begin{array}{r}
4 \\
6 \\
14 \\
16 \\
5 \\
3
\end{array}\right]
$$

Neste caso, a biblioteca identifica que os vetores $z$ e $h$ possuem interseção de memória entre si e efetua a soma em um vetor temporário, para posteriormente atribuir à $z$ o resultado. 


\subsection{Classes Traits}

Classes Traits são classes que complementam funções injetando informações em tempo de compilação. Na biblioteca $S T L$ existem implementadas classes que auxiliam na criação de constantes, classes para obter características de tipos e classes para obter novos tipos aplicando transformações específicas em tipos existentes. Abaixo estão descritas algumas dessas classes.

\section{is fundamental}

Classe que identifica se $T$ é um tipo primitivo.

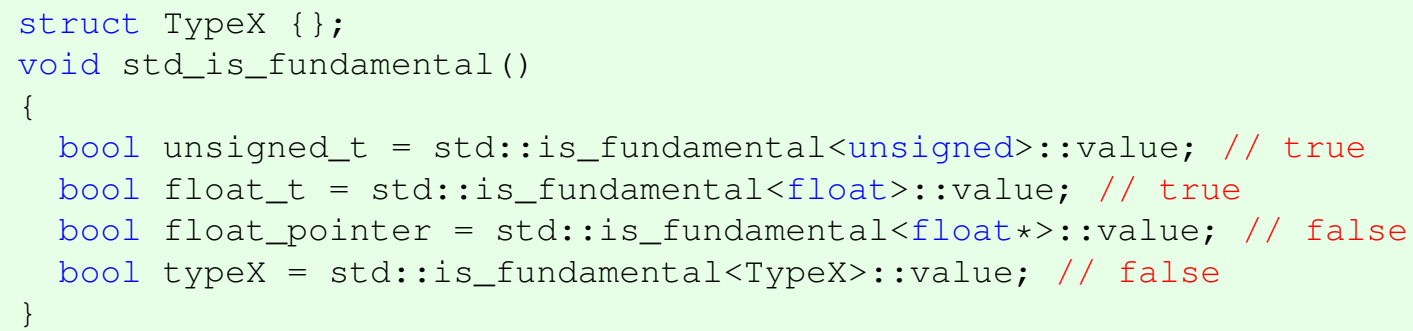

\section{is scalar}

Classe que identifica se $T$ é um escalar. Um escalar é um tipo que possui o operador de soma sem precisar de sobrecarga.

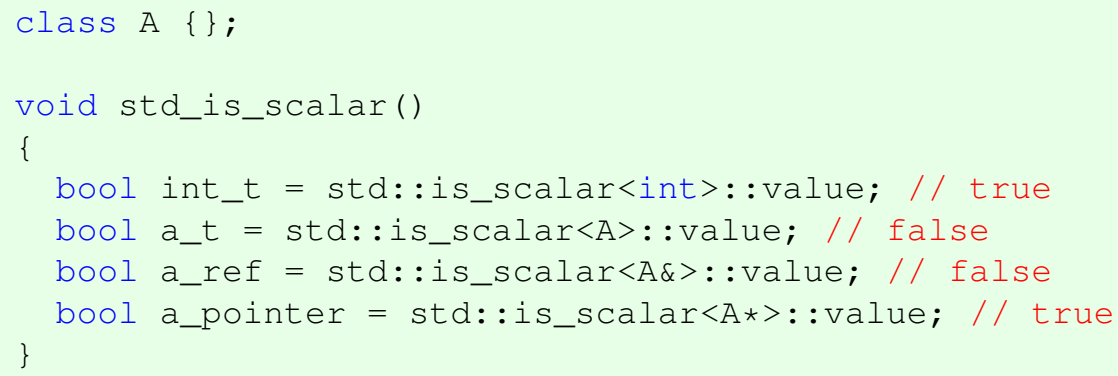

is same

Classe que identifica se $T$ é do mesmo tipo que $U$. Dois tipos com nomes diferentes são considerados do mesmo tipo se, e somente se, um é typedef do outro.

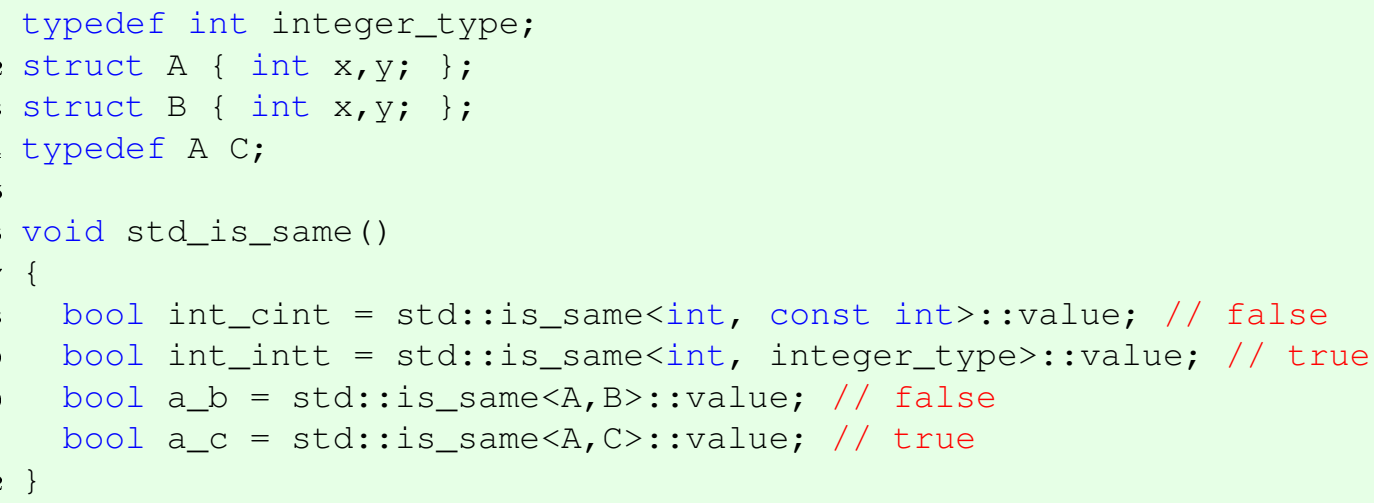

\section{integral constant}

Classe que cria constantes como tipos, em tempo de compilação. Por exemplo, o código abaixo calcula, em tempo de compilação, o fatorial de um número.

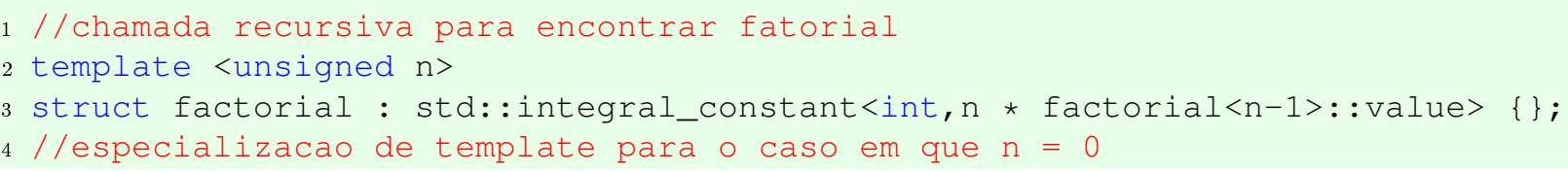




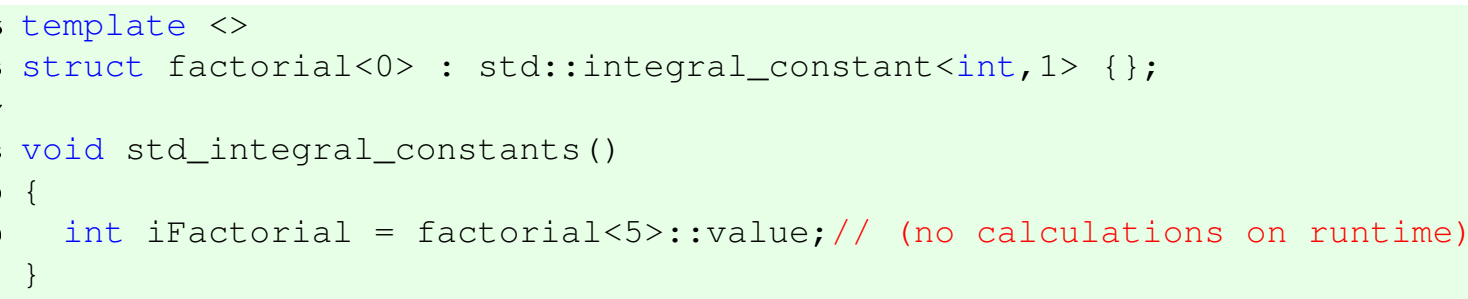

A variável $i$ Factorial receberá o valor $120(5 * 4 * 3 * 2 * 1)$.

\subsubsection{Classes Traits da Klein}

As classes traits foram amplamente utilizadas no código da Klein para evitar que métodos fossem invocados para tipos incoerentes. Em tempo de compilação esses erros são identificados, evitando erros em tempo de execução.

Para cobrir o maior número de casos, foram implementadas classes traits na Klein para serem utilizadas em conjunto com as classes traits da STL. Abaixo descreveremos algumas dessas classes criadas.

\section{is_lazy_vector}

Conforme visto na seção anterior, quando efetuamos operações aritméticas entre vetores é criado um vetor do tipo Lazy. A classe trait is_lazy_vector foi implementada na Klein para verificar se o parâmetro template $\mathrm{T}$ é um vetor lazy. A função is_lazy_vector é escrita dessa forma:

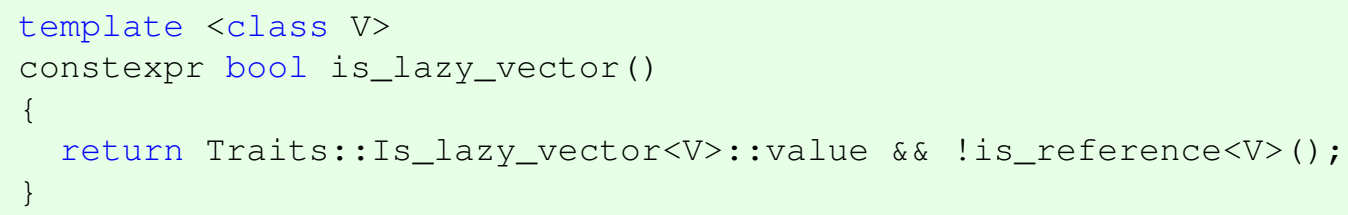

O tipo trait is_reference invocará o método da STL std::is_reference e o tipo Is_lazy_vector retornará falso a não ser que ele seja especializado para retornar true. Como é o caso da classe Vector_plus_vector:

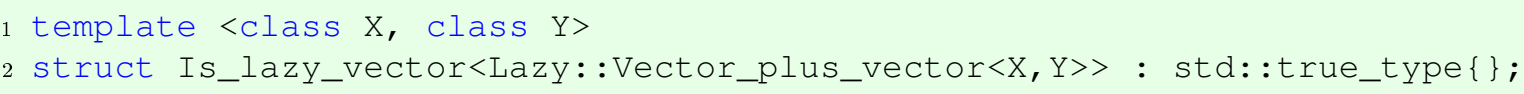

Dessa forma, quando fazemos:

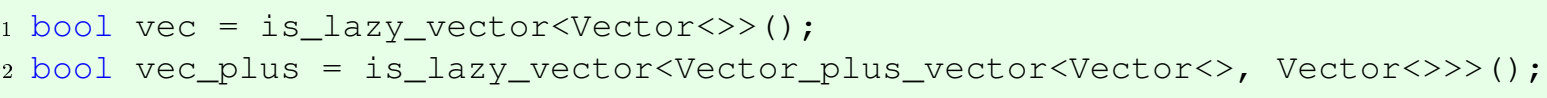

A variável vec receberá o valor false e a variável vec_plus receberá true.

\section{is_lazy_matrix}

Da mesma forma que o item anterior, a função is_lazy_matrix verifica se o parâmetro template $T$ é uma matriz do tipo lazy.

Dessa forma, quando fazemos:

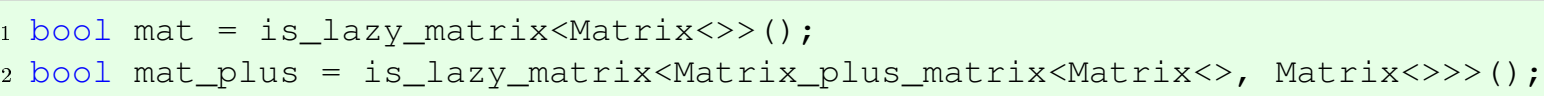

a variável mat receberá o valor false e a variável mat_plus receberá true.

\section{is_rvalue_vector}

A função is_rvalue_vector verifica se o parâmetro template $T$ é um vetor rvalue. Dessa forma, quando fazemos:

1 bool vec = is_rvalue_vector $<$ Vector $<>>()$;

2 bool vec_plus = is_rvalue_vector $\langle$ Vector_plus_vector $<$ Vector $<>$, Vector $<>>>()$;

a variável vec receberá o valor true e a variável vec_plus receberá false. 


\section{is_rvalue_matrix}

A função is_rvalue_matrix verifica se o parâmetro template $T$ é uma matriz rvalue. Dessa forma, quando fazemos:

1 bool mat = is_rvalue_matrix $<$ Matrix $<>>()$;

2 bool mat_plus = is_rvalue_matrix<Matrix_plus_matrix $<$ Matrix $<>$, Matrix $<>>>()$;

a variável mat receberá o valor true e a variável mat_plus receberá false.

\subsubsection{Exemplos de classes traits em funções}

A função abaixo só aceita vetores lazy como parâmetro.

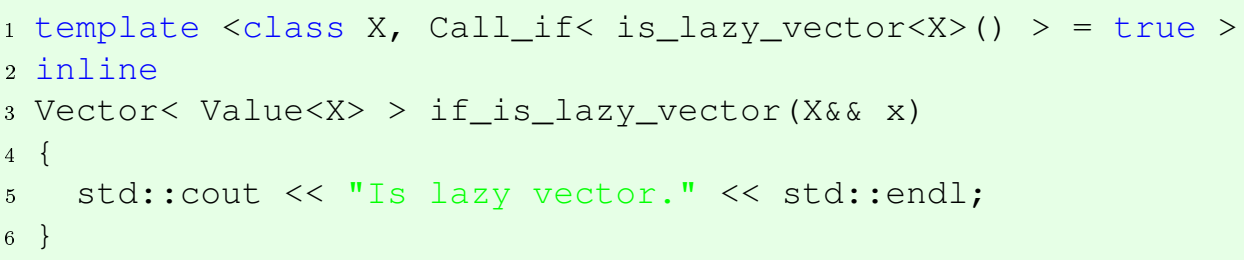

Portanto, ao invocarmos a função dessa forma:

Vector $<>$ vec (5);

2 if_is_lazy_vector(vec);

o código não compila. Para que compile é necessário passar como parâmetro um vetor lazy, conforme abaixo:

1 Vector<> vec(5);

2 if_is_lazy_vector(vec+vec); 


\section{Capítulo 4}

\section{Objetos da Klein}

A Klein implementa os conceitos fundamentais da álgebra linear abstrata, como escalares, vetores e matrizes. Essa implementação é genérica, no sentido que, por exemplo, os escalares podem ser números de ponto flutuante usuais (float e double) e também intervalos, números com maior precisão ou até mesmo números racionais, usados com aritmética exata. Nesta seção apresentamos os principais conceitos implementados pela Klein.

\subsection{Escalares}

Os escalares da Klein são números representados de maneiras diferentes pelo computador. Em $\mathrm{C}++$ temos os tipos float e double, que são primitivos da linguagem. A Klein implementa os tipos Real e Interval que são descritos nesta seção.

\section{Real}

O tipo Real possui dois parâmetros template: o número de bytes do número que será instanciado e o tipo de arredondamento desse número. O tipo de arredondamento default é o arredondamento para o valor mais próximo, porém existem cinco formas de arredondamento:

- $M P F R_{-} R N D N$ : arredondamento para o valor mais próximo.

- $M P F R_{-} R N D Z$ : arredondamento em direção a zero.

- $M P F R_{-} R N D U$ : arredondamento para cima.

- $M P F R_{-} R N D D$ : arredondamento para baixo.

- $M P F R_{-} R N D A$ : arredondamento para longe de zero.

Para um número real de 7x64 bits e arredondamento para cima, podemos instanciá-lo dessa maneira:

1 Real<7, MPFR_RNDU> real (245.890);

Ou podemos definir apenas o número de bytes e deixar o arredondamento default:

1 Real<5> real $(245.890)$;

Os operadores aritméticos foram sobrecarregados para funcionar como nos tipos primitivos da linguagem, então é possível fazermos:

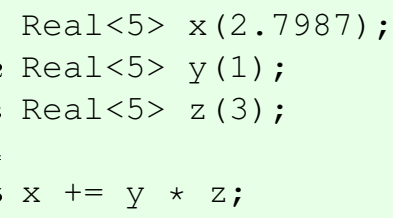




\section{Interval}

O tipo Interval possui dois parâmetros template: o tipo numérico do intervalo e a estratégia de arredondamento. Esse tipo possui como default o tipo numérico double e a estratégia de arredondamento usada nos processadores da Intel. Para alterar a estratégia é necessário especializar a classe Fenv_rounder para a estratégia desejada.

Para um intervalo do tipo double, podemos instanciar dessa forma:

1 Interval<> interval $(0.988,1.769)$;

Os operadores aritméticos foram sobrecarregados para funcionar como nos tipos primitivos da linguagem, então é possível fazermos:

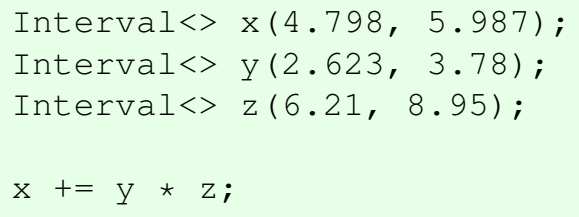

\subsection{Vetores}

Os vetores da Klein possuem como default o tipo primitivo double e podem ser instanciados desta maneira:

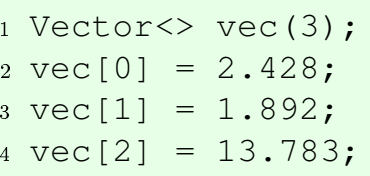

Porém, se for do interesse do programador, é possível a utilização de qualquer tipo primitivo, como inteiros:

1 Vector<int> vec(3);

ou outros tipos numéricos, desde que estes implementem as funcionalidades mínimas exigidas pela Klein. Abaixo encontra-se um exemplo de um vetor sendo instanciado para o tipo Real implementado na Klein:

1 Vector $<$ Real $<4>>\operatorname{vec}(3)$;

\subsection{Matrizes}

As matrizes da Klein podem ser instanciadas para tipos primitivos ou criados pelo próprio usuário. Como default ela instancia o matrizes contendo o tipo double.

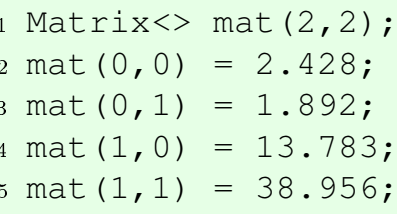

Porém, também podemos instanciar outros tipos primitivos, como inteiros:

1 Matrix<int $>\operatorname{mat}(3,3)$;

ou outros tipos numéricos. Abaixo encontra-se um exemplo de uma matriz sendo instanciada para o tipo Real implementado na Klein 


\subsection{Views}

Na Klein, podemos manipular submatrizes e subvetores através de views. Por exemplo há views que correspondem a um intervalo de elementos de um vetor ou grupos de colunas de uma matriz. A Klein manipula views, matrizes e vetores de forma muito parecida, a única diferença se dá nos construtores e destrutores das classes. Uma view não aloca a memória dos elementos que ela referencia, assim como não destrói esses elementos, visto que eles não pertencem a ela.

Como a view apenas referencia uma sequencia de elementos de um vetor ou uma sequência de colunas de uma matriz, quando alterarmos os valores dos elementos da view, os elementos do vetor ou da matriz também são alterados. Além disso, podemos fazer a atribuição de uma view a um vetor ou a uma matriz e vice versa. Nesses casos são feitas cópias dos elementos de um para o outro. As ações que efetuamos sobre um vetor ou sobre uma matriz, podem ser também efetuadas sobre uma view, visto que são manipulados da mesma forma.

Segue exemplo de código dos casos citados acima para vetores:

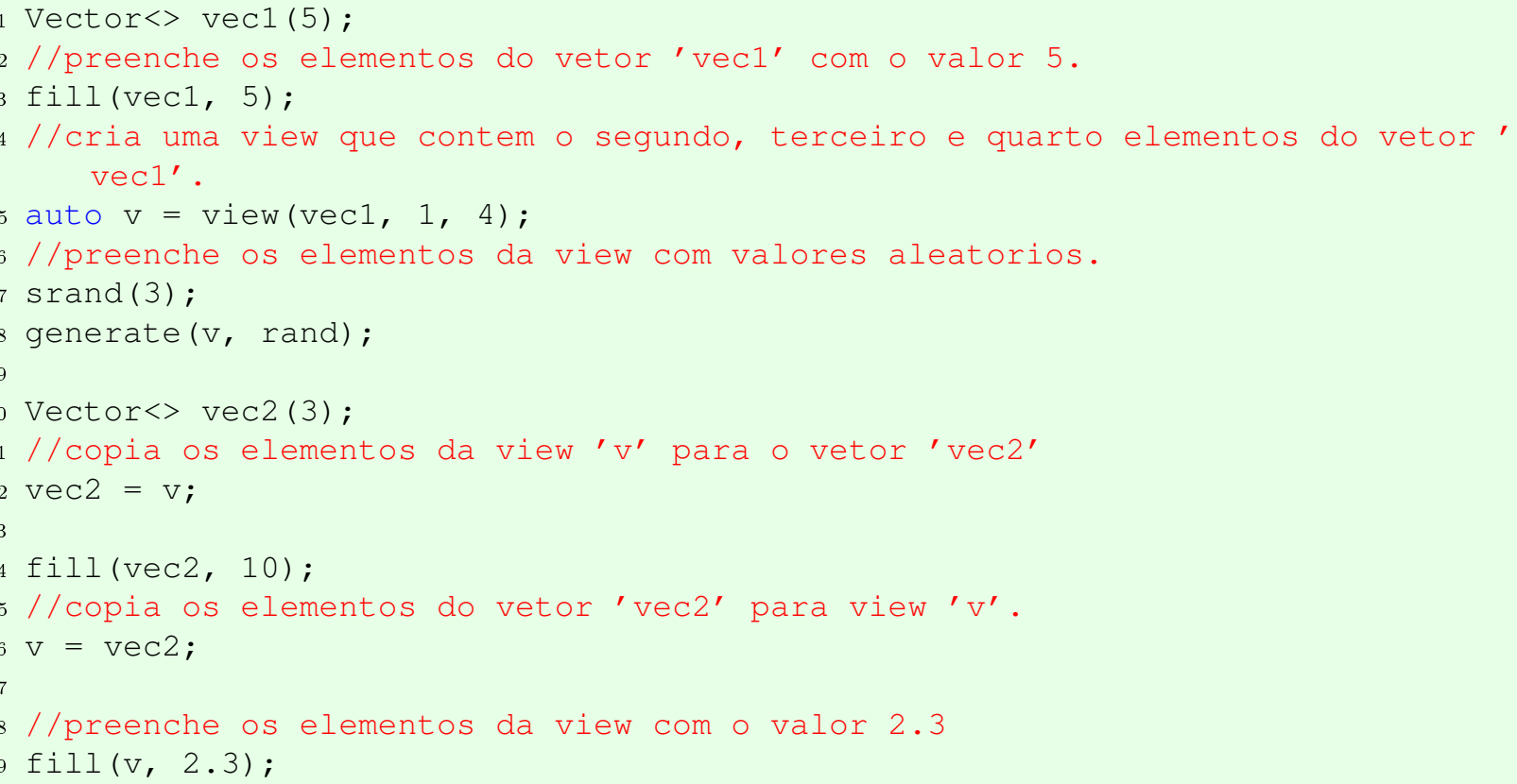

A seguir temos o análogo do código acima para matrizes.

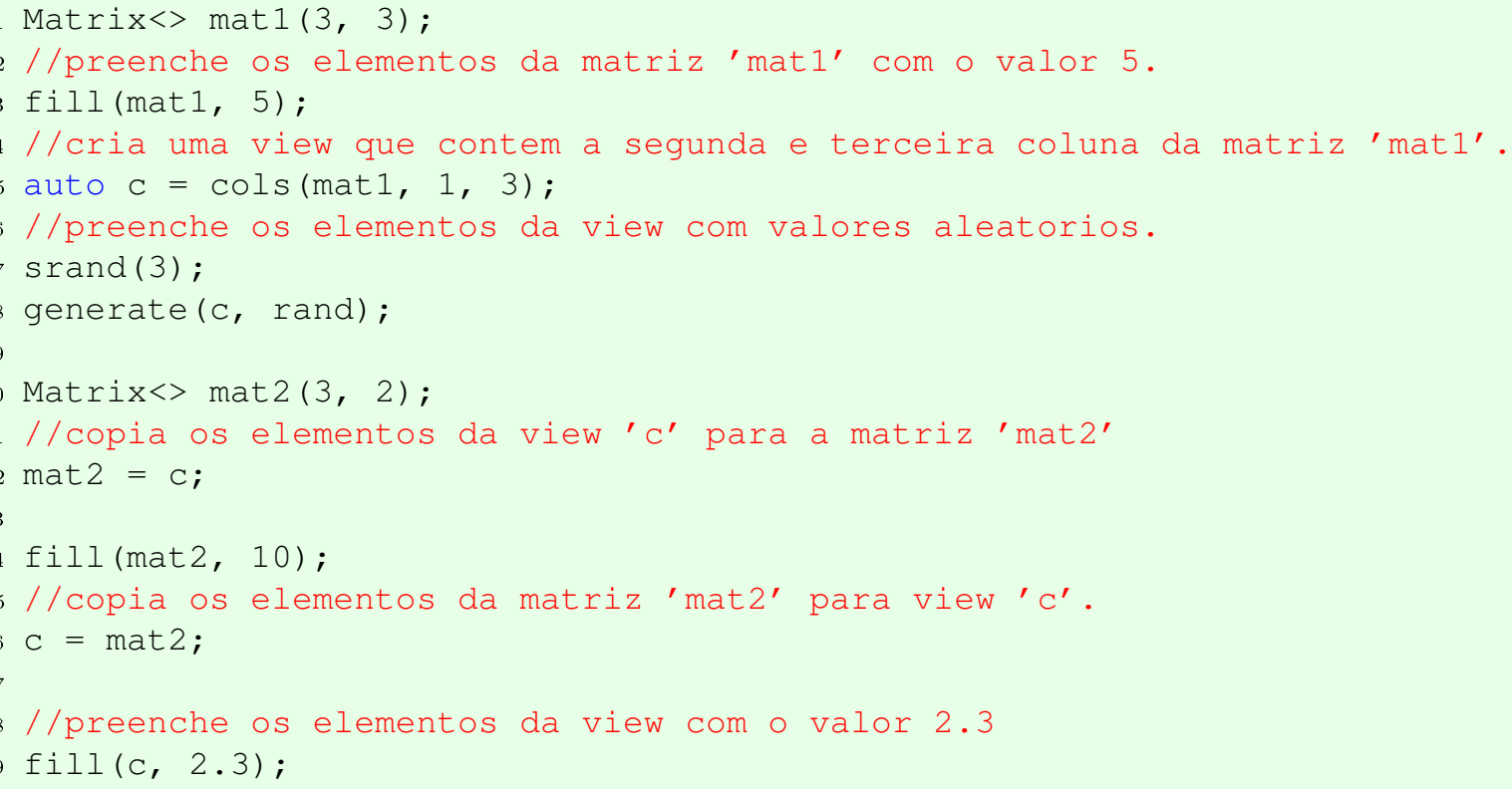




\subsection{Expressões Aritméticas}

A Klein implementa expressões aritméticas envolvendo matrizes e vetores de forma intuitiva. A biblioteca aceita os mesmos operadores utilizados na linguagem $\mathrm{C}++$ para números primitivos como int, double e float. Ou seja, basta utilizarmos os operadores aritméticos,+- e *. Podemos, por exemplo, somar uma matriz com outra e multiplicamos ambas por um escalar, tudo isto em apenas uma linha de código.

\section{Vetores}

Abaixo um exemplo de como efetuar operações aritméticas entre escalares e vetores na Klein.

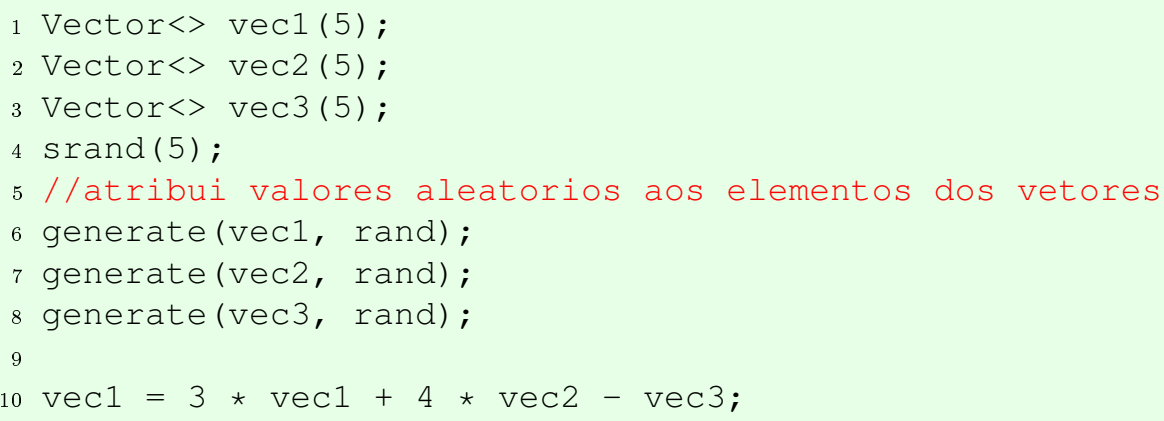

\section{Vetores e views}

Podemos também fazer operações aritméticas com views ao invés de vetores, ou mesclar view e vetor:

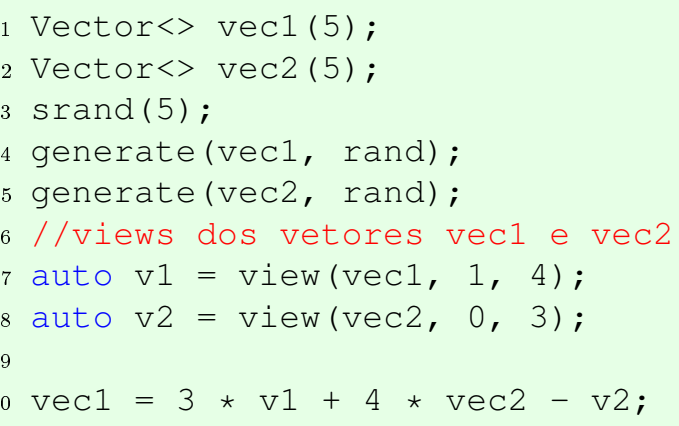

Note que, os vetores possuem cinco elementos, enquanto as views possuem apenas três. As operações aritméticas envolvendo o vetor vec2 serão feitas levando-se em conta apenas os três primeiros elementos do vetor, já que este é o número de elementos das views. Para o vetor vec1 o mesmo ocorre, a atribuição irá ocorrer apenas nos três primeiros elementos.

\section{Matrizes}

Para efetuar operações aritméticas envolvendo matrizes e escalares, podemos fazer dessa forma:

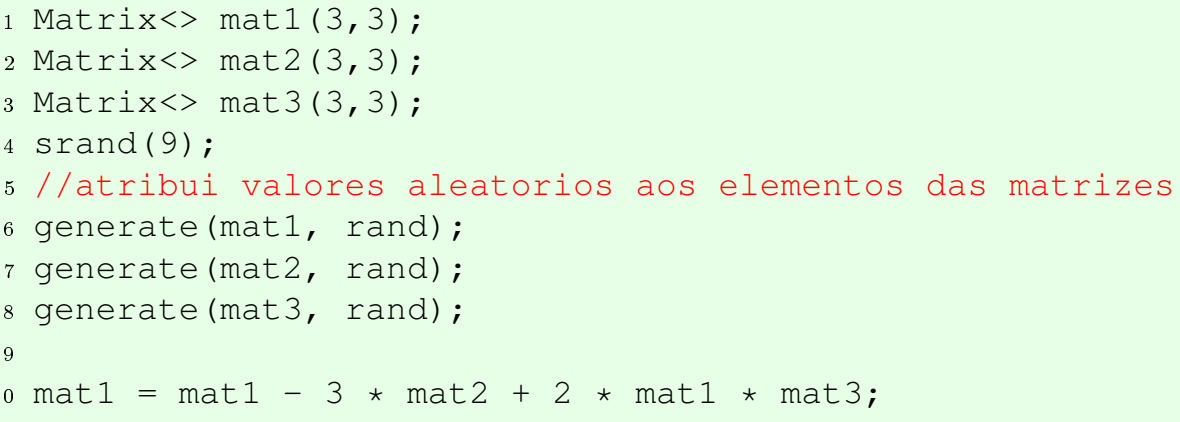

\section{Matrizes e views}

Para utilizar views de matrizes, o código pode ser escrito dessa maneira: 


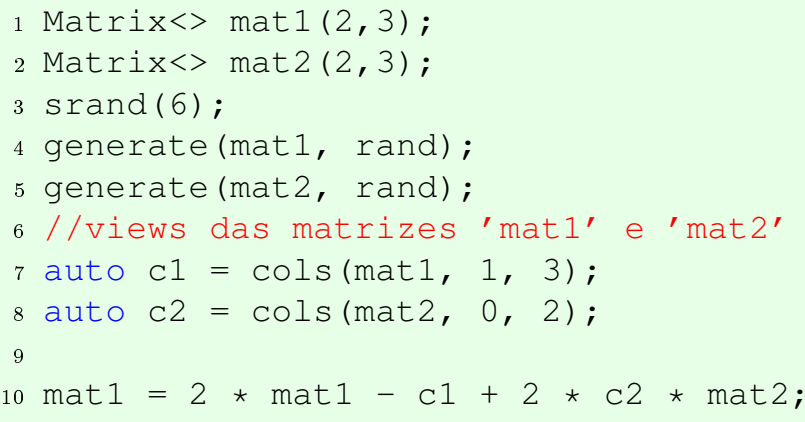

Da mesma forma que os vetores, a soma e subtração das matrizes e views de diferentes tamanhos vão afetar apenas os primeiros elementos da matriz.

\section{Vetores, matrizes e views}

E finalmente, podemos mesclar operações entre escalares, vetores, matrizes e views.

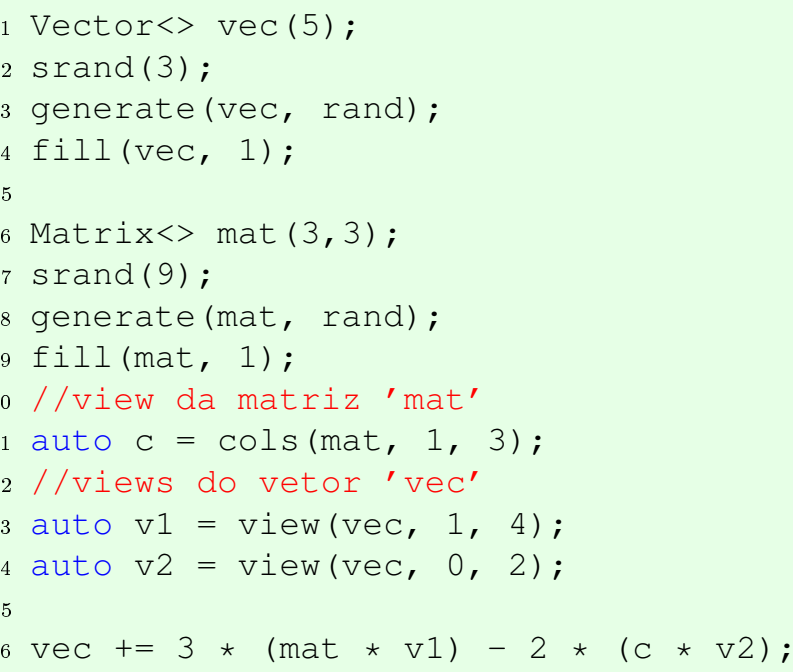

\subsection{Operadores Lineares}

A Klein implementa alguns operadores lineares como permutações, matrizes diagonais, matrizes triangulares e matrizes de Householder.

\section{Permutação de linhas e colunas de matrizes}

Dada uma matriz A, podemos trocar a primeira e segunda linha desta matriz pré multiplicando pela matriz de permutação $\mathrm{P}$.

$$
A=\left[\begin{array}{lll}
a & b & c \\
d & e & f \\
g & h & i
\end{array}\right] \quad P=\left[\begin{array}{lll}
0 & 1 & 0 \\
1 & 0 & 0 \\
0 & 0 & 1
\end{array}\right]
$$

Desta forma, teríamos

$$
P A=\left[\begin{array}{lll}
0 & 1 & 0 \\
1 & 0 & 0 \\
0 & 0 & 1
\end{array}\right]\left[\begin{array}{lll}
a & b & c \\
d & e & f \\
g & h & i
\end{array}\right]=\left[\begin{array}{lll}
d & e & f \\
a & b & c \\
g & h & i
\end{array}\right]
$$

Ou podemos pós multiplicar para trocar a primeira e segunda coluna:

$$
A P=\left[\begin{array}{lll}
a & b & c \\
d & e & f \\
g & h & i
\end{array}\right]\left[\begin{array}{lll}
0 & 1 & 0 \\
1 & 0 & 0 \\
0 & 0 & 1
\end{array}\right]=\left[\begin{array}{lll}
b & a & c \\
e & d & f \\
h & g & i
\end{array}\right]
$$


Na Klein o código para estas permutações fica dessa forma:

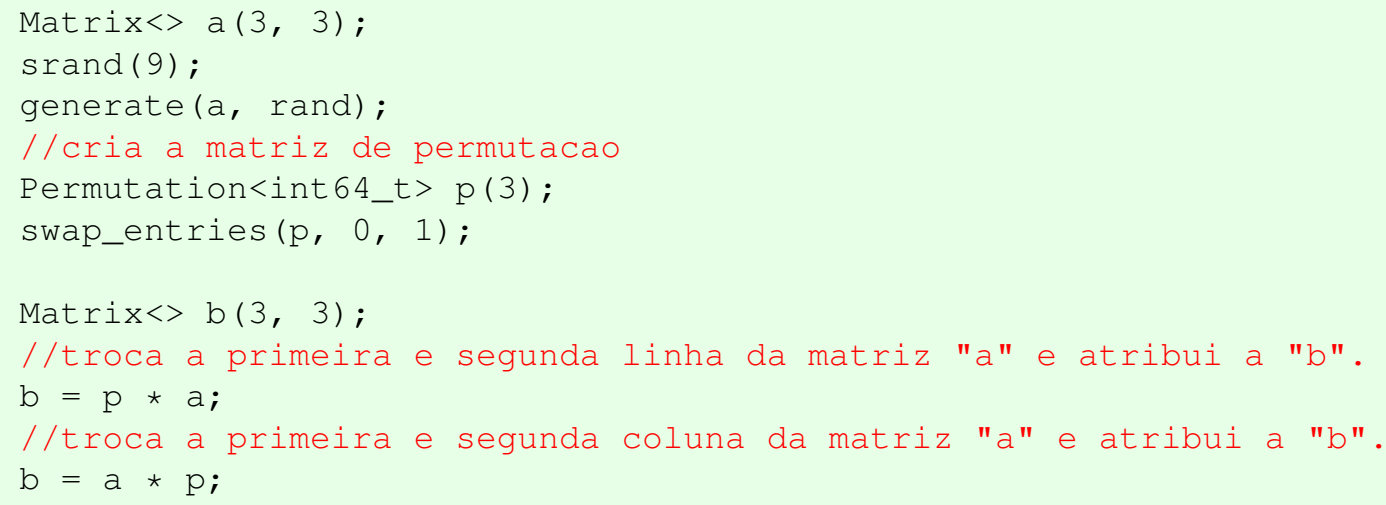

\section{Matriz diagonal}

Uma matriz diagonal é uma matriz que possui elementos nulos fora da diagonal. Por exemplo, a matriz

$$
D=\left[\begin{array}{lll}
a & 0 & 0 \\
0 & b & 0 \\
0 & 0 & c
\end{array}\right]
$$

é diagonal. Como a maioria dos seus elementos são nulos, economizamos memória armazenando somente os elementos da diagonal. Como consequência obtemos um melhor desempenho em operações com estas matrizes, visto que não há necessidade de acessar todos os seus elementos. Na Klein a matriz diagonal armazena os elemetos da diagonal em um vetor, mas para o usuário ela se comporta como uma matriz. A matriz diagonal seria armazenada desta maneira

$$
D=\left[\begin{array}{l}
a \\
b \\
c
\end{array}\right]
$$

Declaramos uma matriz diagonal e preenchemos com elementos aleatórios através do código:

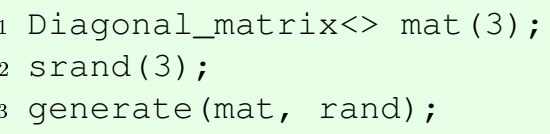

\section{Matriz inversa}

Uma matriz quadrada $A$ possui inversa quando existe outra matriz denotada por $A^{-1}$ tal que: $A^{-1} \cdot A=I$ e $A \cdot A^{-1}=I$ onde $I$ é a matriz identidade.

Por exemplo, uma forma de calcular a inversa da matriz

$$
A=\left[\begin{array}{lll}
3 & 0 & 2 \\
9 & 1 & 7 \\
1 & 0 & 1
\end{array}\right]
$$

seria essa:

$$
\left[\begin{array}{lll}
3 & 0 & 2 \\
9 & 1 & 7 \\
1 & 0 & 1
\end{array}\right] \cdot\left[\begin{array}{lll}
a & b & c \\
d & e & f \\
g & h & i
\end{array}\right]=\left[\begin{array}{lll}
1 & 0 & 0 \\
0 & 1 & 0 \\
0 & 0 & 1
\end{array}\right]
$$


que nos leva ao sistema de equações abaixo:

$$
\left\{\begin{array}{l}
3 a+g=1 \\
3 b+h=0 \\
3 c+i=0 \\
9 a+d+7 g=0 \\
9 b+e+7 h=1 \\
9 c+f+7 i=0 \\
a+g=0 \\
b+h=0 \\
c+i=1
\end{array}\right.
$$

Resolvendo o sistema de equações temos que:

$$
A^{-1}=\left[\begin{array}{rrr}
1 & 0 & -2 \\
-2 & 1 & -3 \\
-1 & 0 & 3
\end{array}\right]
$$

O cálculo da matriz inversa é um processo muito custoso. Por isso, a Klein não calcula a matriz inversa, apenas cria uma estrutura que funciona como se fosse essa matriz inversa. A utilização dessa estrutura é diretamente em expressões, como:

$$
v=v-i n v(a) * v,
$$

onde $v$ é vetor, $a$ é matriz e $\operatorname{inv}(a)$ representa a inversa da matriz:

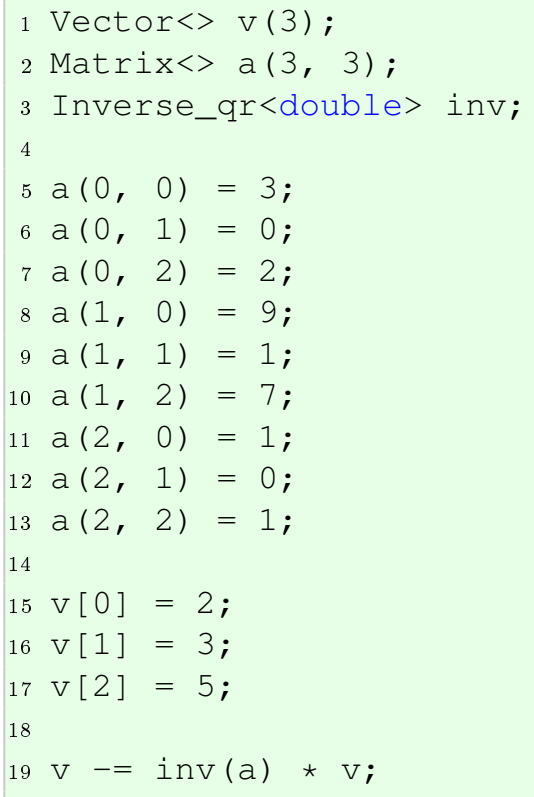

Nesse exemplo o resultado será:

$$
\left[\begin{array}{l}
2 \\
3 \\
5
\end{array}\right]-\left[\begin{array}{rrr}
1 & 0 & -2 \\
-2 & 1 & -3 \\
-1 & 0 & 3
\end{array}\right] \cdot\left[\begin{array}{l}
2 \\
3 \\
5
\end{array}\right]=\left[\begin{array}{r}
10 \\
19 \\
-8
\end{array}\right]
$$




\section{Transformação de vetor em matriz diagonal}

O operador! transforma um vetor com $n$ elementos em uma matriz diagonal $n x n$. Por exemplo, ao utilizarmos o operador ! no vetor

$$
v e c=\left[\begin{array}{l}
2 \\
3 \\
5 \\
8
\end{array}\right]
$$

conforme código abaixo:

1 mat $=$ ! vec;

Teríamos como resultado a matriz:

$$
\text { mat }=\left[\begin{array}{llll}
2 & 0 & 0 & 0 \\
0 & 3 & 0 & 0 \\
0 & 0 & 5 & 0 \\
0 & 0 & 0 & 8
\end{array}\right]
$$

Este operador serve para fazermos o produto entre dois vetores, elemento a elemento. Por exemplo, se $c, a$ e $b$ são vetores, o código:

$1 \mathrm{c}=! \mathrm{a} \star \mathrm{b}$;

tem como resultado

$$
\left[\begin{array}{c}
a 1 \\
a 2 \\
a 3 \\
a 4 \\
\ldots
\end{array}\right]\left[\begin{array}{l}
b 1 \\
b 2 \\
b 3 \\
b 4 \\
\ldots
\end{array}\right]=\left[\begin{array}{c}
a 1 . b 1 \\
a 2 . b 2 \\
a 3 . b 3 \\
a 4 . b 4 \\
\ldots
\end{array}\right]=\left[\begin{array}{c}
c 1 \\
c 2 \\
c 3 \\
c 4 \\
\ldots
\end{array}\right]
$$

\section{Transformação de matriz em vetor}

O operador vec transforma uma matriz com $n x m$ elementos em um vetor com $n x m$ elementos. As colunas do vetor são colocadas de forma sequencial na memória. Por exemplo, ao utilizarmos o operador vec na matriz

$$
\text { mat }=\left[\begin{array}{lll}
2 & 4 & 7 \\
9 & 3 & 6 \\
1 & 8 & 5
\end{array}\right]
$$

conforme código abaixo:

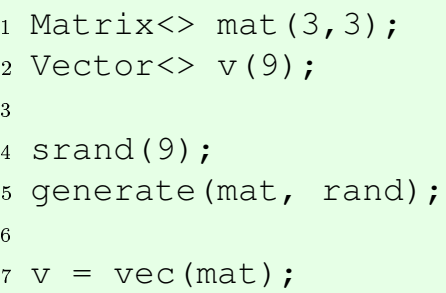

Teríamos como resultado o vetor:

$$
v e c=\left[\begin{array}{c}
2 \\
9 \\
1 \\
4 \\
3 \\
8 \\
7 \\
6 \\
5
\end{array}\right]
$$




\subsection{Decomposições}

As principais decomposições de matrizes foram implementadas na Klein afim de facilitar a criação de algoritmos e simplificá-los. Abaixo serão descritos como utilizar as decomposições LU, QR e SVD.

\section{Decomposição LU}

A decomposição LU consiste da decomposição de uma matriz $A$ não singular como o produto de uma matriz triangular inferior $L$ e uma matriz triangular superior $U$.

$$
A=L U
$$

Dado o sistema

$$
A x=b,
$$

podemos resolvê-lo utilizando a decomposição LU da seguinte forma:

$$
\begin{gathered}
A=L U \\
L U x=b .
\end{gathered}
$$

O primeiro passo é resolver

$$
L y=b,
$$

onde queremos encontrar o valor de y. Depois, resolvemos

$$
U x=y
$$

e encontramos o valor de $x$.

Além disso, a Klein permite o uso de pivotamento parcial e completo. Ou seja, também temos as fatorações $P A=L U$ e $P A T=L U$, onde $P$ e $T$ são matrizes de permutação.

Com a Klein, podemos resolver o sistema $A x=b$, pela decomposição $L U$, através do código abaixo:

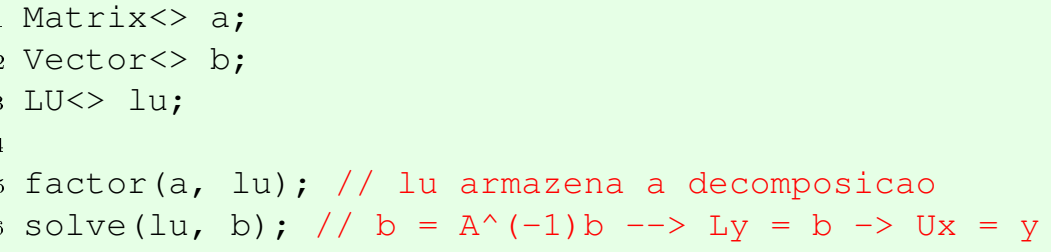

\section{Decomposição QR}

A decomposição QR consiste da decomposição de uma matriz A como o produto de uma matriz ortogonal $Q$ por uma matriz triangular superior $R$.

$$
A=Q R
$$

Dada a equação $A x=b$, podemos resolvê-la utilizando a decomposição QR da seguinte forma:

$$
A^{T} A x=A^{T} b
$$

Como $(Q R)^{T}=R^{T} Q^{T}$ podemos reescrever:

$$
R^{T} Q^{T} Q R x=R^{T} Q^{T} b .
$$

Sendo Q ortogonal, $\left(Q^{T} Q=I\right)$, então

$$
R^{T} R x=R^{T} Q^{T} b
$$




$$
R x=Q^{T} b
$$

Primeiro encontramos as matrizes Q e R:

$$
A=Q R
$$

depois calculamos $\mathrm{d}$ :

$$
d=Q^{T} b
$$

e por último resolvemos

$$
R x=d
$$

Com a Klein, podemos resolver o sistema $A x=b$, pela decomposição $Q R$, através do código abaixo:

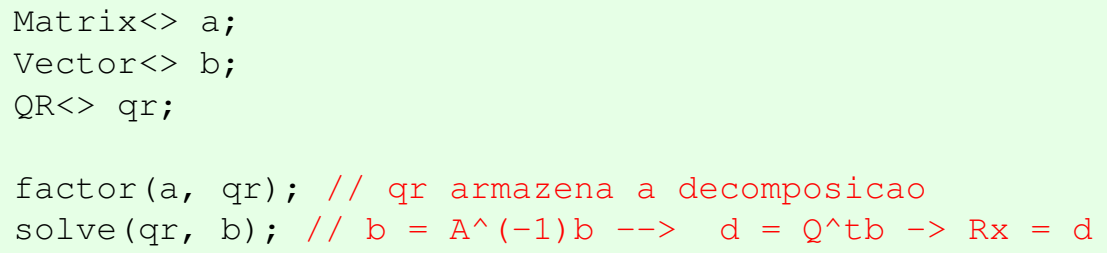

\section{Decomposição SVD}

A decomposição SVD consiste da decomposição de uma matriz $A$ como o produto de duas matrizes ortogonais, $U$ e $V$, e uma matriz diagonal $\Sigma$.

$$
A=U \Sigma V^{t}
$$

Dado o sistema

$$
A x=b,
$$

podemos resolvê-lo utilizando a decomposição SVD da seguinte forma:

$$
\begin{gathered}
A x=b \\
U \Sigma V^{t} x=b \\
x=V \Sigma^{-1} U^{t} b \\
w=U^{t} b \\
x=V \Sigma^{-1} w
\end{gathered}
$$

Com a Klein, podemos resolver o sistema $A x=b$, pela decomposição $S V D$, através do código abaixo:

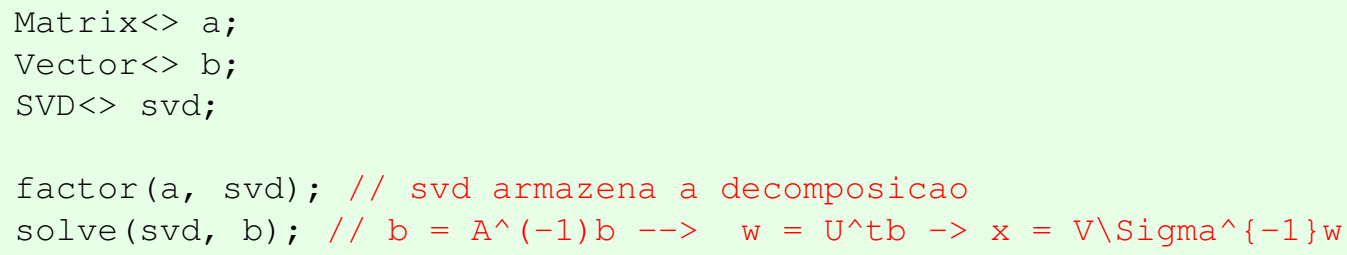

\subsection{Algoritmos}

A Klein implementa alguns algoritmos importantes para álgebra linear como fill, que preenche os elementos de um vetor com um único valor, sin, que calcula o seno de cada elemento de um vetor e retorna o resultado em outro vetor, max, que retorna o maior elemento de uma matriz, dentre outros que serão descritos no próximo capítulo. 


\section{Capítulo 5}

\section{Algoritmos e funções da Klein}

\subsection{Algoritmos}

\subsubsection{Algoritmos da STL}

A Klein implementa alguns algoritmos para manipular matrizes e vetores análogos aos da biblioteca STL, porém de forma mais simples e intuitiva. Por exemplo para aplicar a função $f(x)=2 x+3$ nos elementos de um vetor e atribuir à outro utilizando a STL, teríamos que invocar o método transfom passando os seguintes parâmetros:

- first1: a posição inicial do vetor a ser transformado.

- last1: a posição final do vetor a ser transformado.

- d_first: a posição inicial do vetor destino.

- unary_op: a função que será aplicada sobre os elementos do vetor.

enquanto que para invocar o método da Klein seriam necessários os parâmetros abaixo:

- dest: o vetor destino.

- vec: o vetor a ser transformado.

- unary_op: a função que será aplicada sobre os elementos do vetor.

O código utilizando cada uma das bibliotecas fica desta forma:

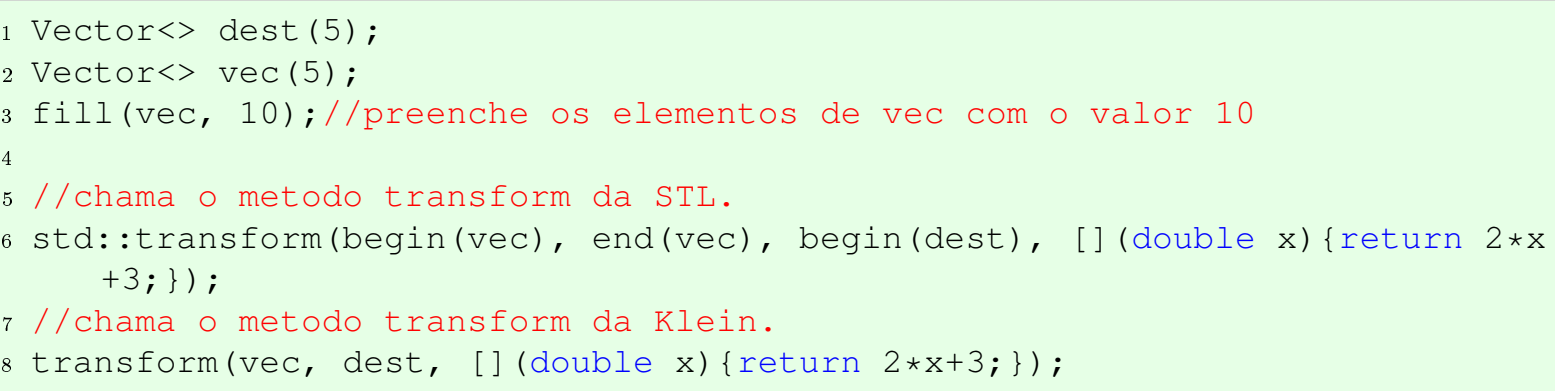

Abaixo serão descritos os métodos implementados na Klein, já existentes na STL.

fill

Podemos preencher parcialmente um vetor ou matriz através da função fill, que recebe os seguintes parâmetros:

- dest: view a ser preenchida.

- value: valor que será atribuído aos elementos de dest. 


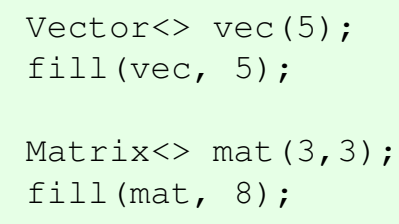

Resultado:

$$
v e c=\left[\begin{array}{c}
5 \\
5 \\
5 \\
5 \\
5
\end{array}\right] \quad \text { mat }=\left[\begin{array}{lll}
8 & 8 & 8 \\
8 & 8 & 8 \\
8 & 8 & 8
\end{array}\right]
$$

Para uma view de um vetor ou matriz poderíamos fazer:

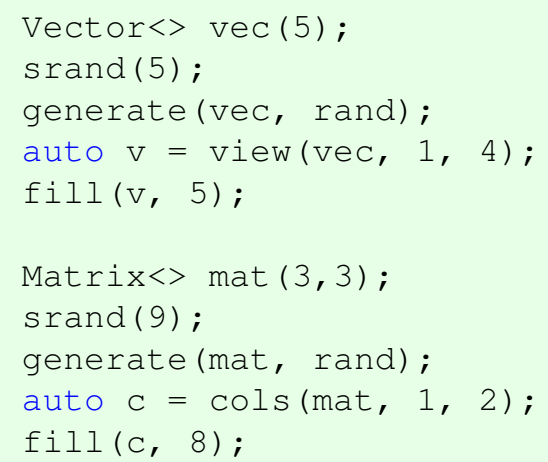

Resultado:

$$
v e c=\left[\begin{array}{c}
3.235 \\
5 \\
5 \\
5 \\
2.87
\end{array}\right] \quad \text { mat }=\left[\begin{array}{lll}
1.24 & 8 & 2.43 \\
8.61 & 8 & 7.35 \\
4.36 & 8 & 5.27
\end{array}\right]
$$

\section{generate}

Podemos preencher matrizes e vetores através da função generate. Para isto, basta chamarmos a função passando os parâmetros:

- vec: vetor a ser preenchido.

- func: função que irá gerar os valores dos elementos. Esta função não recebe parâmetros.

Se desejarmos preencher um vetor e uma matriz com valores aleatórios, podemos utilizar a função rand da biblioteca padrão do $\mathrm{C}$, conforme abaixo:

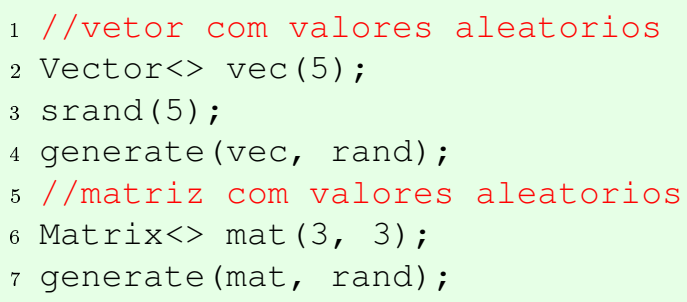


Neste caso, podemos utilizar no lugar na função rand qualquer outra função que não possui parâmetro, mas que retorne o tipo esperado pelos objetos de destino. Resultado:

$$
v e c=\left[\begin{array}{c}
33.235 \\
15.73 \\
53.234 \\
25.32 \\
2.87
\end{array}\right] \quad \text { mat }=\left[\begin{array}{ccc}
1.24 & 10.23 & 2.43 \\
8.61 & 23.42 & 7.35 \\
4.36 & 121.54 & 5.27
\end{array}\right]
$$

Também poderíamos gerar valores apenas para partes do vetor ou da matriz através do código abaixo:

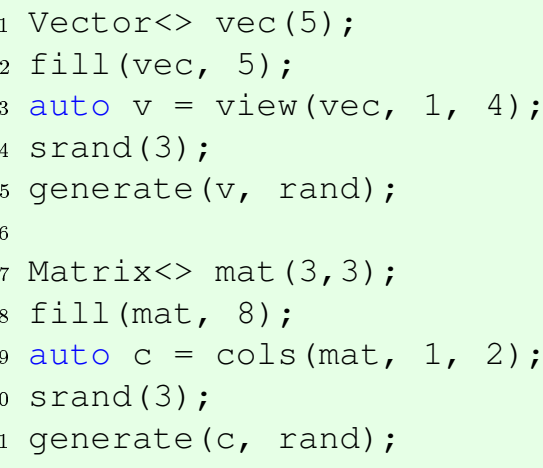

Resultado:

$$
\text { vec }=\left[\begin{array}{c}
5 \\
15.73 \\
53.234 \\
25.32 \\
5
\end{array}\right] \quad \text { mat }=\left[\begin{array}{ccc}
8 & 10.23 & 8 \\
8 & 23.42 & 8 \\
8 & 121.54 & 8
\end{array}\right]
$$

\section{transform}

Para preencher matrizes e vetores através de uma função unária, podemos utilizar a função transform, que retorna a transformação de um vetor através de uma função passada como parâmetro. Este método possui duas assinaturas. Uma que recebe os parâmetros:

- vec: vetor a ser transformado e retornado.

- func: função que irá transformar os elementos do vetor vec. Esta função deve receber um parâmetro de mesmo tipo dos elementos de vec.

A outra assinatura tem parâmetros:

- vec1: vetor a ser preenchido.

- vec2: vetor a ser transformado.

- func: função que irá transformar os elementos do vetor vec2. Esta função deve receber um parâmetro de mesmo tipo dos elementos de vec2.

Caso nosso interesse seja criar vetores ou matrizes com valores em função dos valores dos vetores ou matrizes faríamos da seguinte forma:

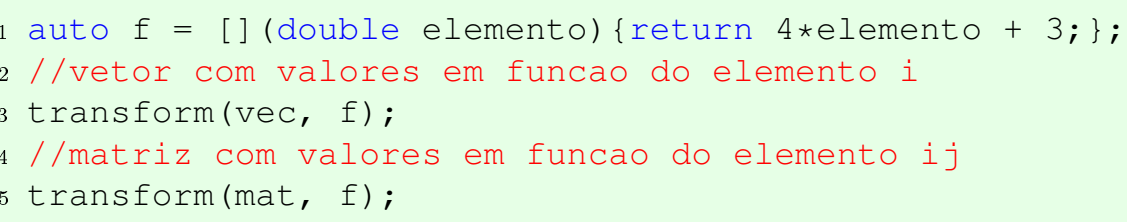


O vetor vec receberá na posição de índice i o valor $4 * v e c[i]+3$, de acordo com a função $\mathrm{f}$. Assim como os elementos da matriz, que receberão na posição i,j o valor $4 * \operatorname{mat}(i, j)+3$.

$$
\operatorname{vec}=\left[\begin{array}{l}
4 * \operatorname{vec}[0]+3 \\
4 * \operatorname{vec}[1]+3 \\
4 * \operatorname{vec}[2]+3 \\
4 * \operatorname{vec}[3]+3 \\
4 * \operatorname{vec}[4]+3
\end{array}\right] \quad \operatorname{mat}=\left[\begin{array}{lll}
4 * \operatorname{mat}(0,0)+3 & 4 * \operatorname{mat}(0,1)+3 & 4 * \operatorname{mat}(0,2)+3 \\
4 * \operatorname{mat}(1,0)+3 & 4 * \operatorname{mat}(1,1)+3 & 4 * \operatorname{mat}(1,2)+3 \\
4 * \operatorname{mat}(2,0)+3 & 4 * \operatorname{mat}(2,1)+3 & 4 * \operatorname{mat}(2,2)+3
\end{array}\right]
$$

Além destes, podemos preencher um vetor ou uma matriz em função de valores de outros vetores e matrizes, respectivamente, conforme segue:

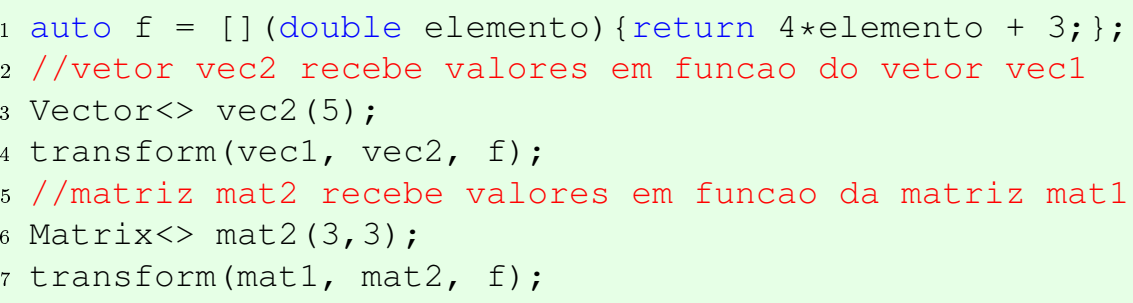

Neste exemplo o primeiro elemento do vetor vec 2 receberá o valor $2 * v e c 1[0]$ e o primeiro elemento da matriz receberá o valor $4 * \operatorname{mat} 1(0,0)+3$.

$\operatorname{vec} 2=\left[\begin{array}{l}4 * \operatorname{vec} 1[0]+3 \\ 4 * \operatorname{vec} 1[1]+3 \\ 4 * \operatorname{vec} 1[2]+3 \\ 4 * \operatorname{vec} 1[3]+3 \\ 4 * \operatorname{vec} 1[4]+3\end{array}\right] \quad$ mat $2=\left[\begin{array}{lll}4 * \operatorname{mat} 1(0,0)+3 & 4 * \operatorname{mat} 1(0,1)+3 & 4 * \operatorname{mat} 1(0,2)+3 \\ 4 * \operatorname{mat} 1(1,0)+3 & 4 * \operatorname{mat} 1(1,1)+3 & 4 * \operatorname{mat} 1(1,2)+3 \\ 4 * \operatorname{mat} 1(2,0)+3 & 4 * \operatorname{mat} 1(2,1)+3 & 4 * \operatorname{mat} 1(2,2)+3\end{array}\right]$.

\subsubsection{Algoritmos de Estatística}

A Klein implementa algoritmos para obter estatísticas dos elementos dos vetores e das matrizes de forma simplificada. Assim, a média dos valores de um vetor pode ser calculada com uma única chamada de função, por exemplo. Abaixo descrevemos as funções existentes.

$\max$

Para encontrar o maior elemento de um vetor ou de uma matriz, basta utilizarmos a função max. Ela retorna o valor do maior elemento e recebe como parâmetro:

- src: objeto do qual desejamos obter o maior valor.

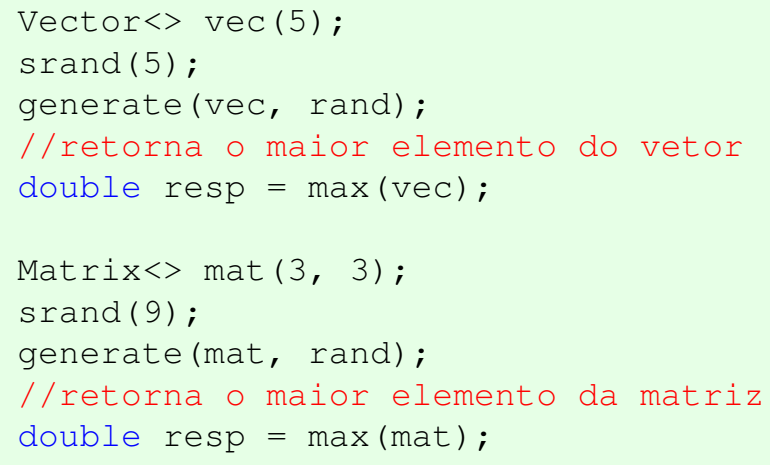

Para encontrar o menor elemento de um vetor ou de uma matriz, basta utilizarmos a função min. Ela retorna o valor do menor elemento e recebe como parâmetro:

- src: objeto do qual desejamos obter o menor valor. 


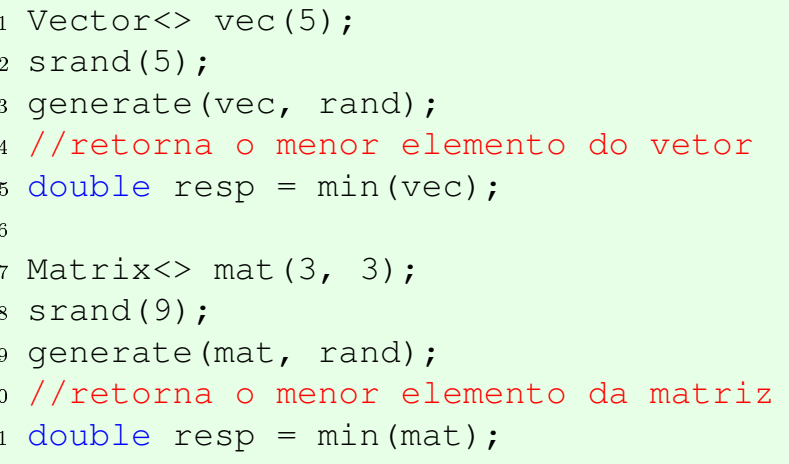

\section{sum}

A função sum calcula a soma de todos os elementos de um vetor ou de uma matriz. Ela retorna o valor da soma dos elementos do vetor ou da matriz e recebe como parâmetro:

- src: objeto a ser calculada a soma de seus elementos.

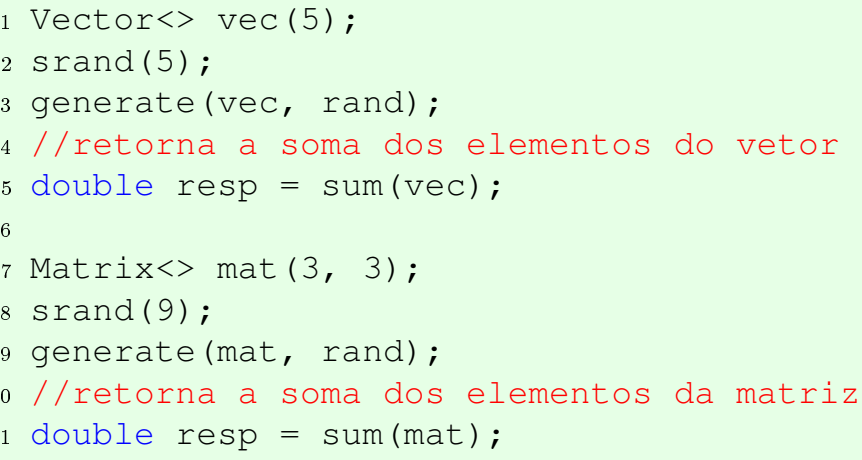

\section{are near}

Para verificar se os elementos entre dois vetores ou entres duas matrizes são parecidos ou iguais, podemos utilizar a função are_near. Esta função recebe os parâmetros:

- src1: primeiro objeto a ser comparado.

- src2: segundo objeto a ser comparado.

- tol: valor de tolerância. Este campo permite uma tolerância na comparação entre os valores dos elementos dos objetos sendo comparados

Para verificar se os valores de dois vetores e de duas matrizes são iguais ou parecidos fazemos da seguinte forma:

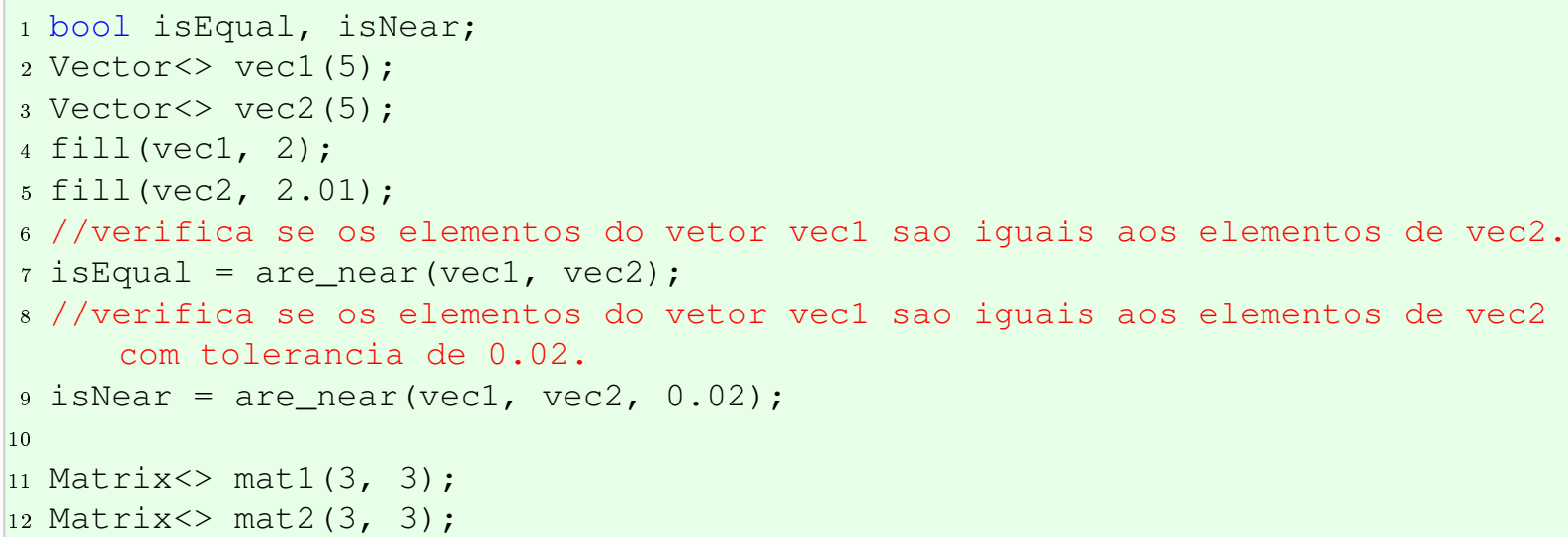




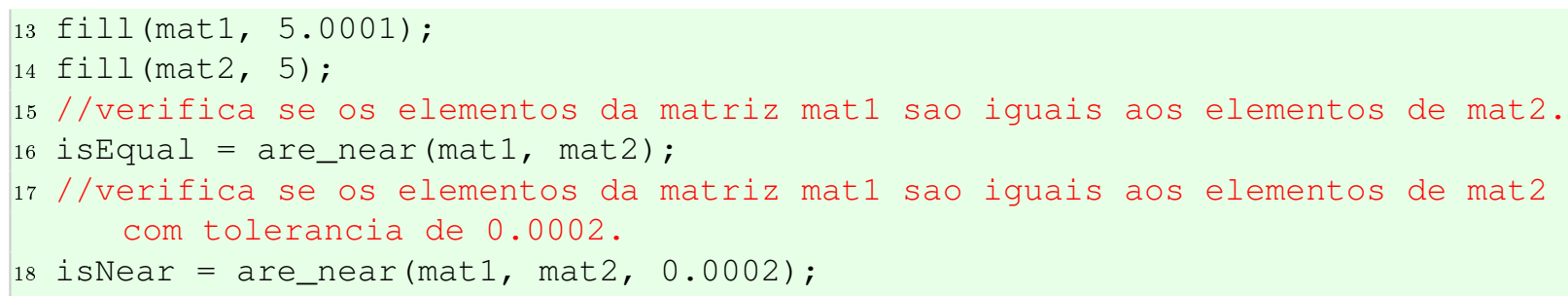

dot

Para fazer o produto interno dos elementos de um vetor, podemos utilizar a função dot. Esta função recebe os parâmetros:

- src1: primeiro vetor a ser multiplicado.

- src2: segundo vetor a ser multiplicado.

Para fazer o produto interno entre dois vetores fazemos da seguinte forma:

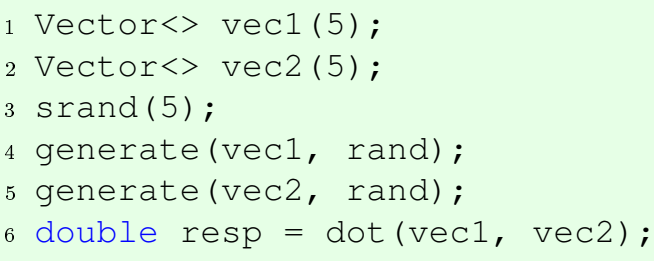

\section{$\max$ dist}

Para encontrar a maior diferença entre os elementos de dois vetores ou duas matrizes, utilizamos a função max_dist. Esta função recebe os parâmetros:

- src1: primeiro objeto a ser comparado.

- src2: segundo objeto a ser comparado.

Exemplo:

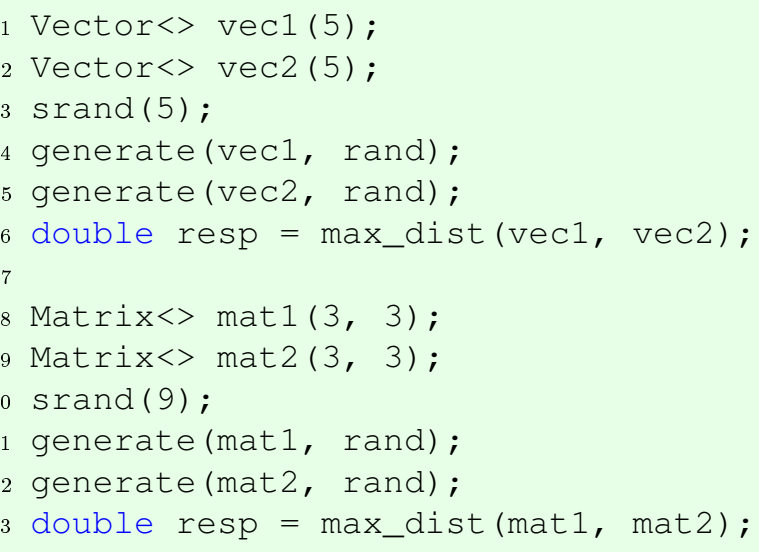

Para encontrar o maior elemento em módulo de um vetor ou de uma matriz, utilizamos a função max_abs. Esta função recebe os parâmetros:

- src: vetor ou matriz a ser analisada.

Exemplo: 


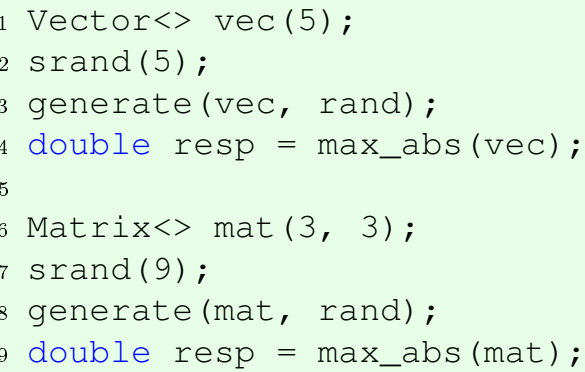

\section{mean}

Para calcular a média dos elementos de um vetor, utilizamos a função mean. Esta função recebe o parâmetro:

- src: vetor cuja média de seus elementos será calculada.

Exemplo:

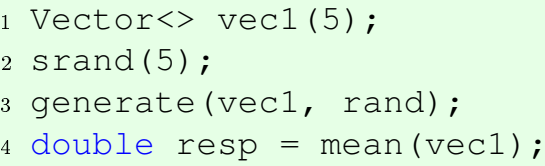

\section{col means}

Para calcular as médias dos elementos das colunas de uma matriz, utilizamos a função col_means. Esta função recebe o parâmetro:

- src: matriz cujas médias dos elementos das colunas serão calculadas.

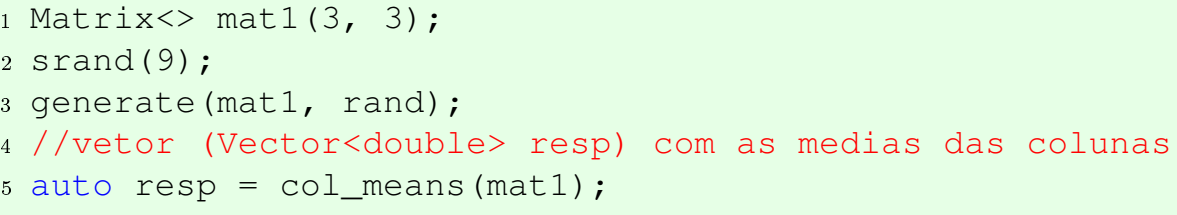

Para calcular o desvio padrão dos elementos de um vetor, utilizamos a função std_dev. Esta função recebe o parâmetro:

- src: vetor cujo desvio padrão de seus elementos será calculado.

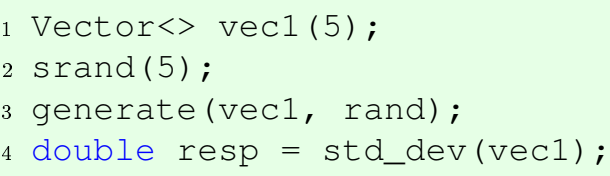

Para calcular os desvios padrões dos elementos das colunas de uma matriz, utilizamos a função col_means. Esta função recebe o parâmetro:

- src: matriz que queremos calcular os desvios padrões dos elementos das colunas.

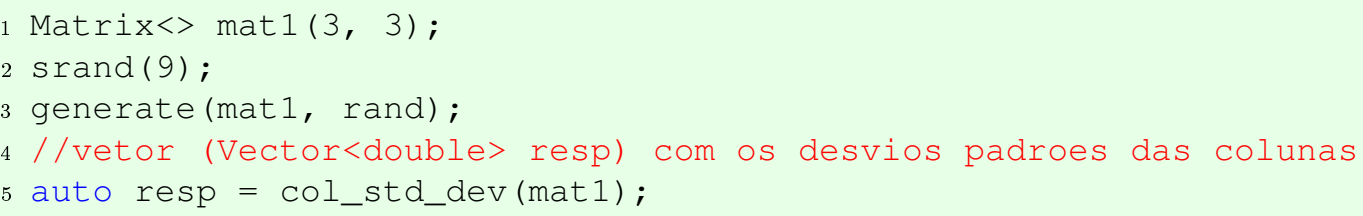




\section{two norm}

A função two_norm calcula a norma de um vetor através da seguinte fórmula:

$$
\operatorname{vec}[i]=\sqrt{\sum \operatorname{vec}[i]^{2}}
$$

Esta função recebe como parâmetro:

- src: vetor a ser calculada a norma.

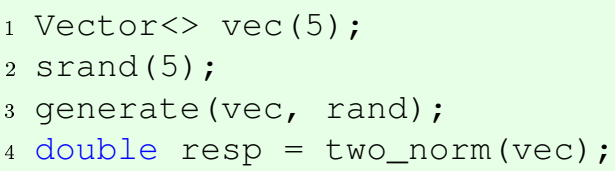

\section{zScore}

Para calcular o quanto do desvio padrão os elementos de um vetor estão acima da média, utilizamos a função zscore. O cálculo é dado pela fórmula:

$$
x[i]=\frac{x[i]-\operatorname{mean}(x)}{s t d \_\operatorname{dev}(x)} .
$$

A função recebe os parâmetros:

- src: vetor a ser calculado o z-score.

- size: tamanho do vetor a ser calculado.

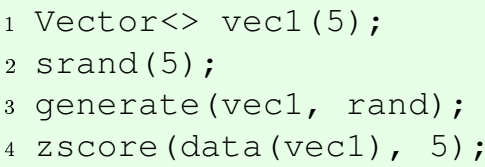

\section{non zeros}

A função non_zeros conta o número de elementos diferentes de zero do vetor ou da matriz. Ela retorna o número de elementos não zeros e recebe como parâmetro:

- src: um vetor ou uma matriz.

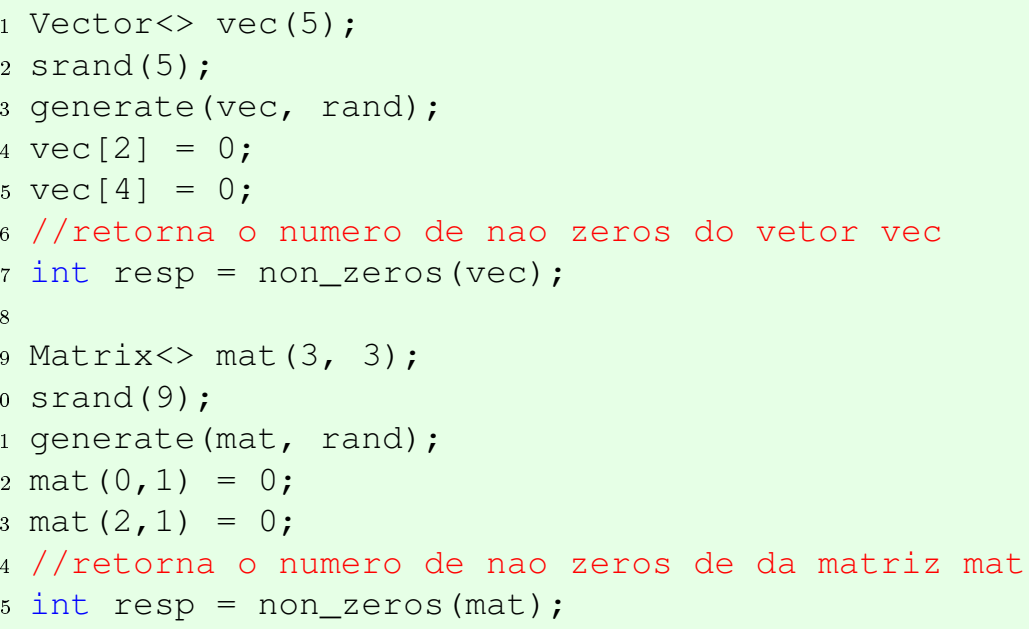




\section{frob_norm}

A função frob_norm calcula a norma de Frobenius da matriz. Esta norma é dada pela fórmula:

$$
\|A\|_{F}=\sqrt{\sum_{i=1}^{m} \sum_{j=1}^{n}\left|a_{i j}\right|^{2}} .
$$

A função retorna o cálculo da norma de Frobenius e recebe como parâmetro:

- src: matriz a ser calculada a norma de Frobenius.

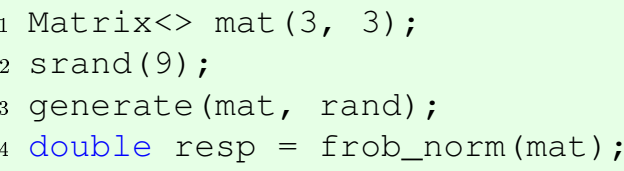

\subsubsection{Outros Algoritmos}

Além destes foram implementados outros algoritmos de propósito geral, que se encontram descritos abaixo:

\section{zero}

Podemos preencher matrizes e vetores com zero através da função zero, que recebe o seguinte parâmetro:

- dest: vetor a ser preenchido com zero.

Por exemplo, o código abaixo preenche um vetor e uma matriz com zeros:

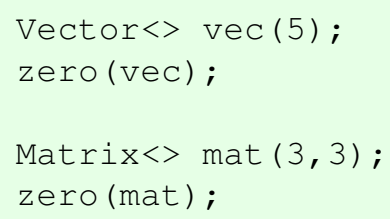

Resultado:

$$
v e c=\left[\begin{array}{l}
0 \\
0 \\
0 \\
0 \\
0
\end{array}\right] \quad \text { mat }=\left[\begin{array}{lll}
0 & 0 & 0 \\
0 & 0 & 0 \\
0 & 0 & 0
\end{array}\right]
$$

Podemos também fazer esta atribuição a uma view de um vetor ou matriz:

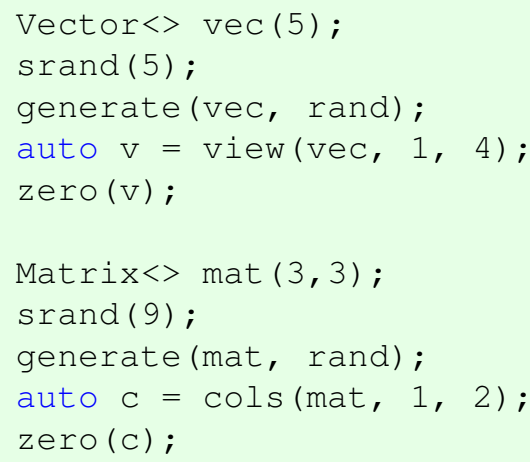

Resultado:

$$
\text { vec }=\left[\begin{array}{c}
3.235 \\
0 \\
0 \\
0 \\
2.87
\end{array}\right] \quad \text { mat }=\left[\begin{array}{lll}
1.24 & 0 & 2.43 \\
8.61 & 0 & 7.35 \\
4.36 & 0 & 5.27
\end{array}\right]
$$


O mesmo se aplica a todas as funções desta seção.

\section{copy_data}

Para copiar elementos entre matrizes e entre vetores, podemos utilizar a função copy_data. Esta função recebe os parâmetros:

- source: vetor ou matriz a ser copiado.

- dest: vetor ou matriz destino.

Para copiar os valores de um vetor e de uma matriz faríamos da seguinte forma:

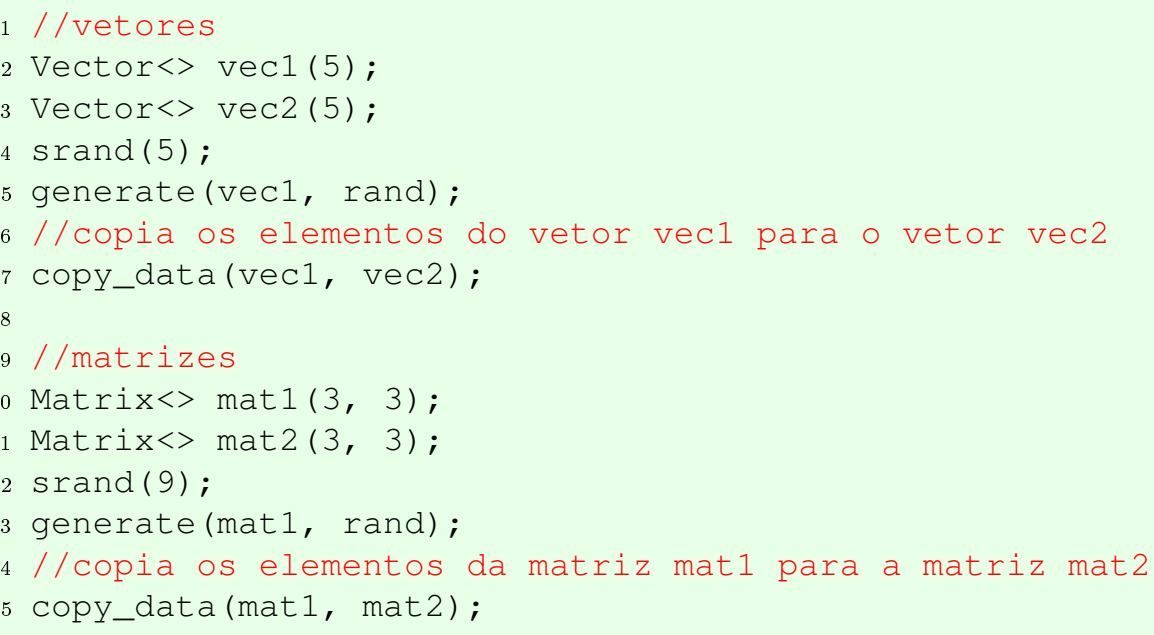

\section{floor}

A função floor retorna o piso dos elementos de um vetor ou de uma matriz. Esta função retorna um vetor e recebe como parâmetro:

- source: vetor ou matriz cujo piso de seus elementos será retornado.

Para retornar o piso dos elementos de um vetor e de uma matriz fazemos dessa forma:

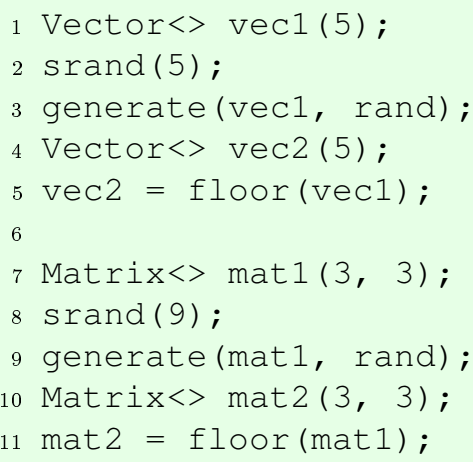

$\sin$

A função sin calcula o seno dos elementos de um vetor ou de uma matriz. Esta função retorna um vetor e recebe como parâmetro:

- source: vetor ou matriz cujo seno de seus elementos será calculado.

Para calcular o seno dos elementos de um vetor fazemos dessa forma:

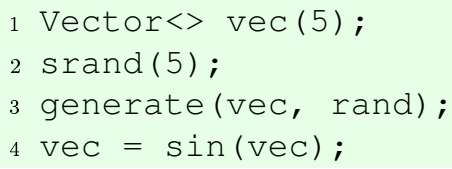


6 Matrix< $\operatorname{mat}(3,3)$;

7 srand (9);

8 generate (mat, rand);

9 mat $=\sin ($ mat $)$;

$\cos$

A função $\cos$ calcula o cosseno dos elementos de um vetor ou de uma matriz. Esta função retorna um vetor e recebe como parâmetro:

- source: vetor ou matriz cujo cosseno de seus elementos será calculado.

Para calcular o cosseno dos elementos de um vetor fazemos dessa forma:

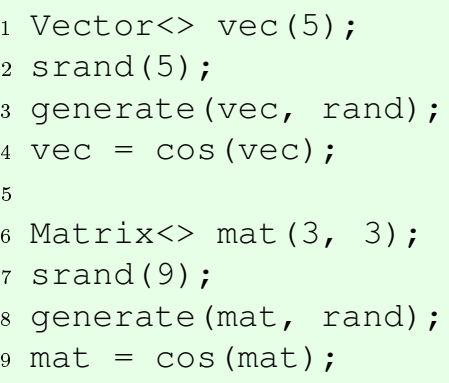

\subsection{Funções básica de álgebra linear (BLAS)}

As funções básica de álgebra linear (BLAS) são divididas em três partes. A primeira, consiste das funções de interação entre vetores (Blas1). A segunda, das funções de interação entre matriz e vetor (Blas2). E a terceira, que contém as funções de interação entre matrizes (Blas3).

Abaixo descrevemos, algumas dessas funções que foram implementadas na Klein.

\subsubsection{Blas1}

sum

A função sum faz o cálculo:

$$
\text { result }=\sum_{i=0}^{n-1} x[i],
$$

onde $y$ e $x$ são vetores e $a$ é escalar.

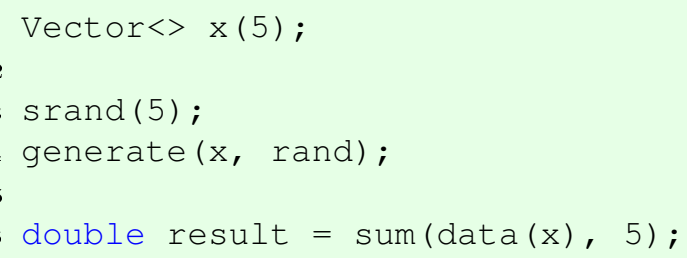

abs

A função $a b s$ faz o cálculo:

$$
x[i]=\operatorname{fabs}(x[i])
$$

onde $x$ é vetor.

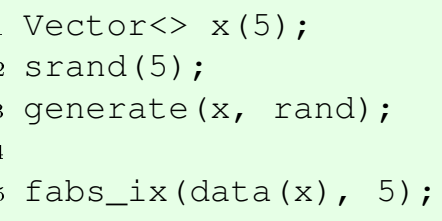


dot

A função dot faz o cálculo:

$$
\text { result }=\sum_{i=0}^{n-1} x[i] * y[i]
$$

onde $x$ e $y$ são vetores e result é escalar.

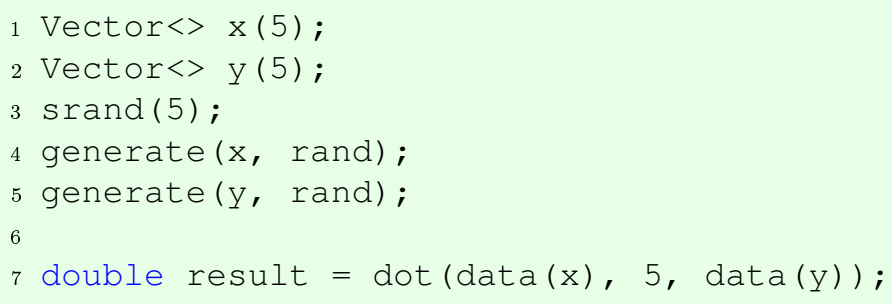

sub

A função $s u b$ faz o cálculo:

$$
z=x-y,
$$

onde $x, y$ e $z$ são vetores.

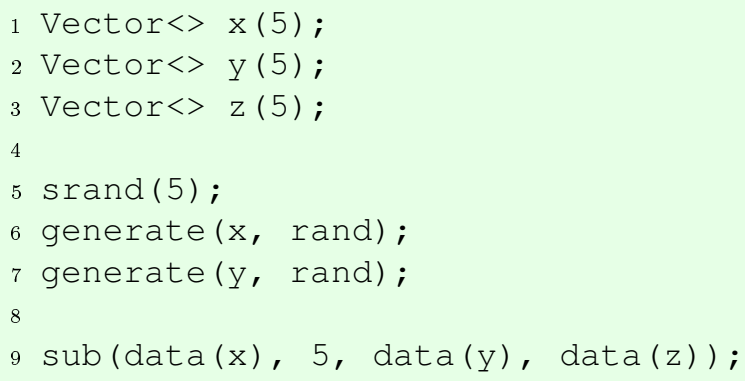

sub ix

A função $s u b \_i x$ faz o cálculo:

$$
x=x-y,
$$

onde $x$ e $y$ são vetores.

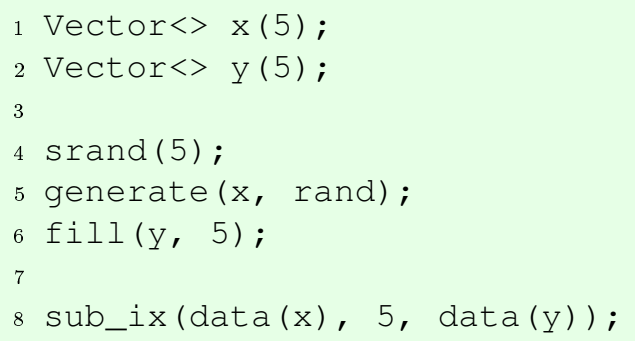

sub_iy

A função $s u b \_i y$ faz o cálculo:

$$
y=x-y,
$$

onde $x$ e $y$ são vetores.

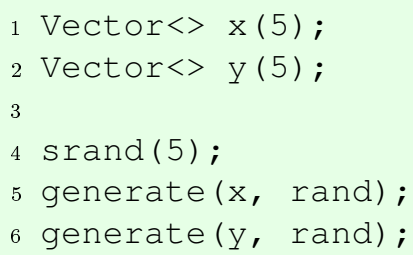


add

A função $a d d$ faz o cálculo:

$$
z=x+y,
$$

onde $x, y$ e $z$ são vetores.

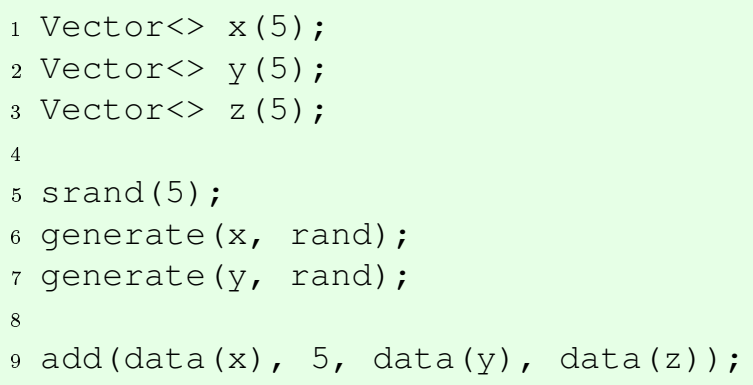

div

A função div faz o cálculo:

$$
z=x / y
$$

onde $x, y$ e $z$ são vetores.

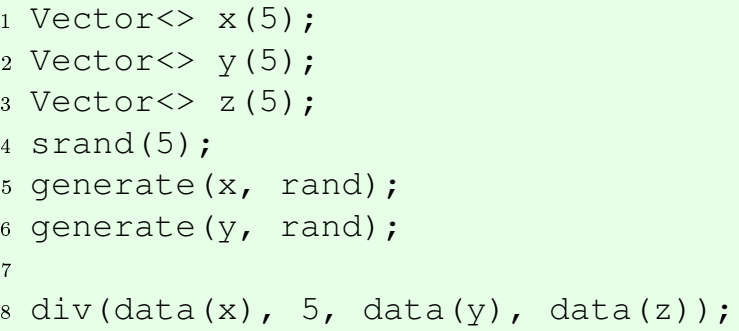

dive

A função divc faz o cálculo:

$$
y=x / a,
$$

onde $x$ e $y$ são vetores e $a$ é escalar.

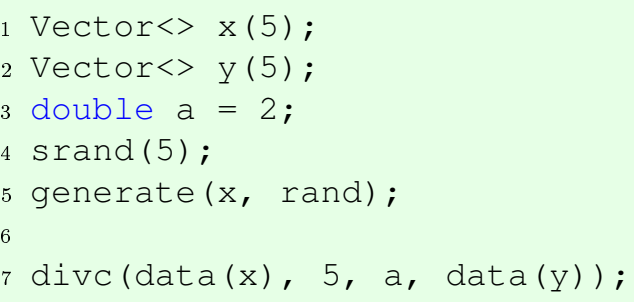

A função rev_divc faz o cálculo:

$$
y=a / x,
$$

onde $x$ e $y$ são vetores e $a$ é escalar.

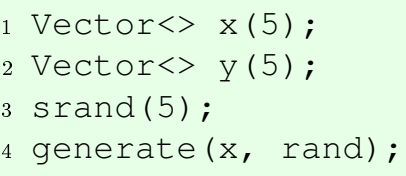




\section{mul}

A função mul faz o cálculo:

$$
z=x * y,
$$

onde $x, y$ e $z$ são vetores.

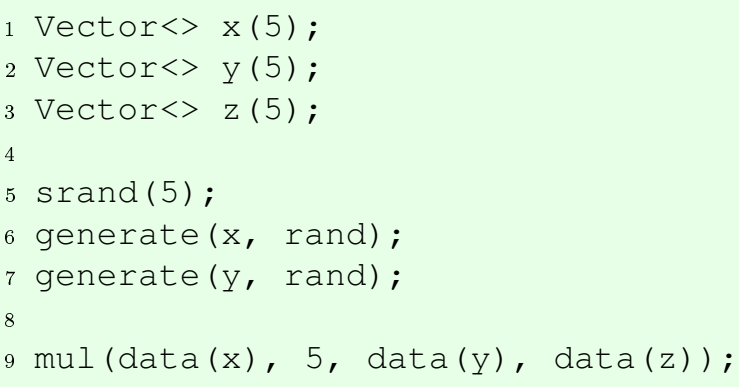

mul_iy

A função mul_iy faz o cálculo:

$$
y=x * y,
$$

onde $x$ e $y$ são vetores.

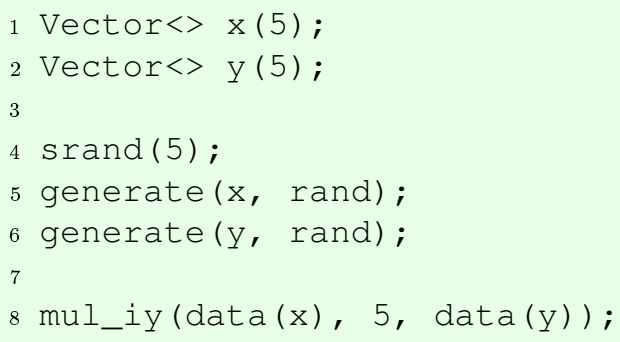

\section{mul add}

A função mul_add faz o cálculo:

$$
w=x * y+z
$$

onde $x, y, z$ e $w$ são vetores.

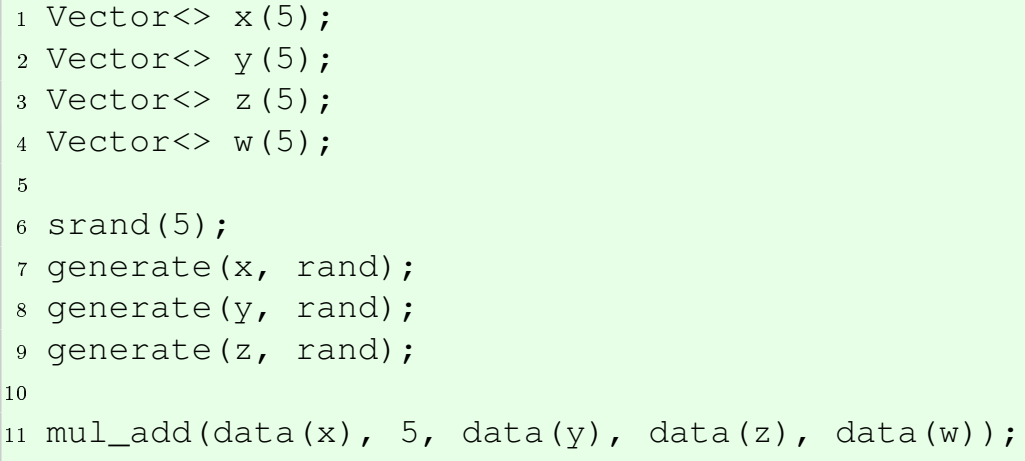

A função $m u l \_a d d_{-} i z$ faz o cálculo:

$$
z=x * y+z
$$

onde $x, y$ e $z$ são vetores. 


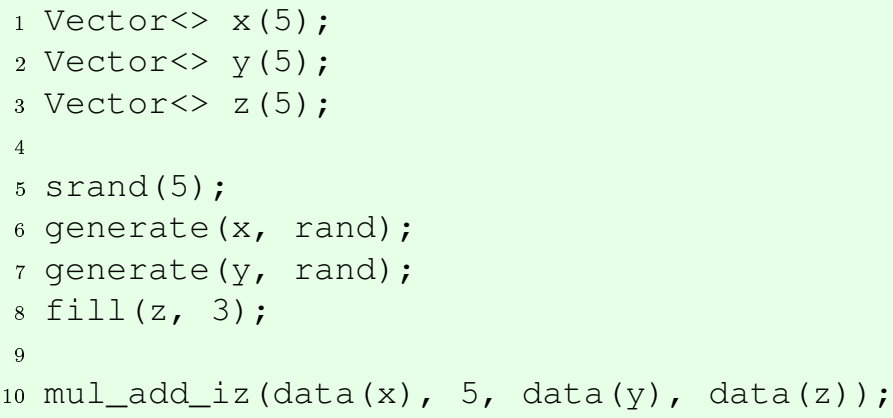

\section{mul sub}

A função mul_sub faz o cálculo:

$$
w=x * y-z,
$$

onde $x, y, z$ e $w$ são vetores.

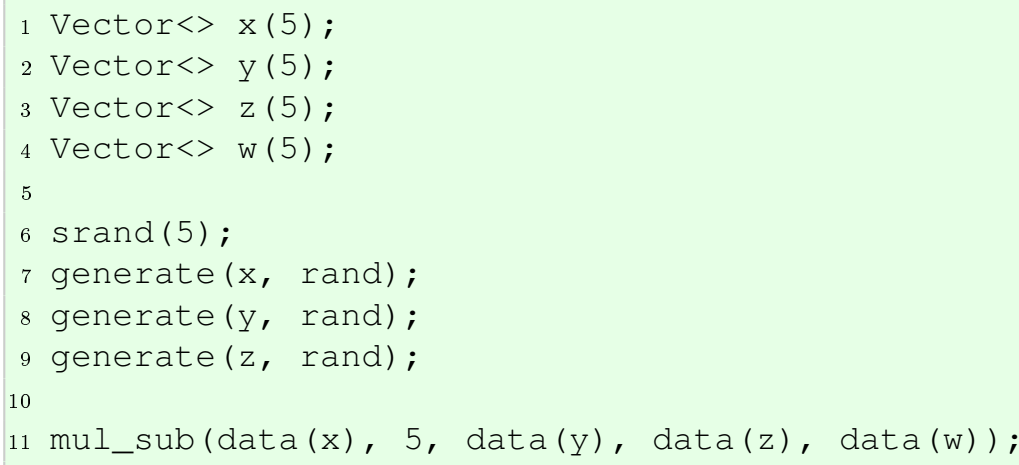

A função mul_rev_sub faz o cálculo:

$$
w=z-x * y,
$$

onde $x, y, z$ e $w$ são vetores.

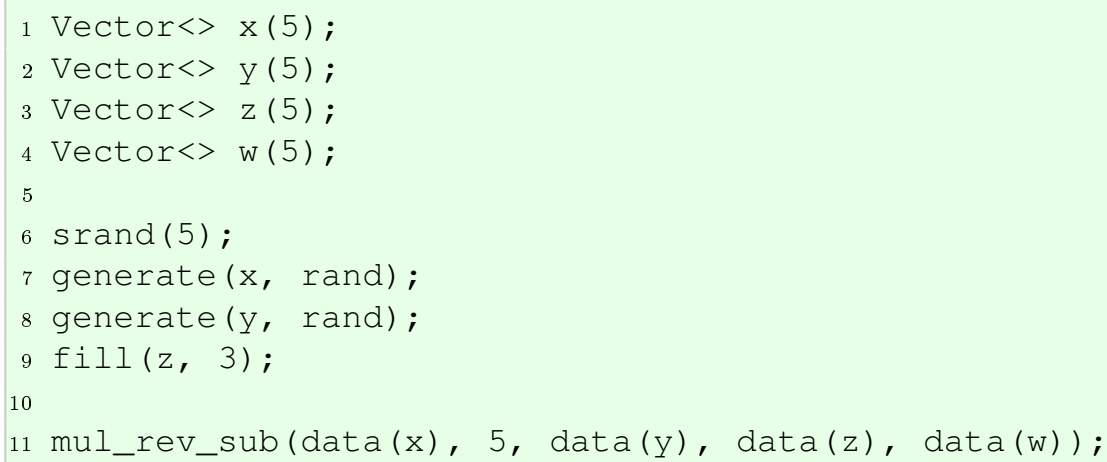

mul_rev_sub_iz

A função $m u l_{-} r e v_{-} s u b_{-} i z$ faz o cálculo:

$$
z=z-x * y
$$

onde $x, y$ e $z$ são vetores.

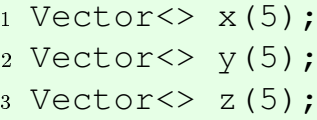




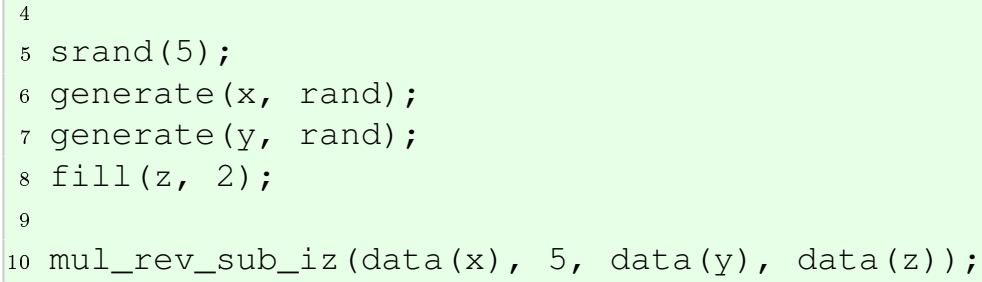

mulc

A função mulc faz o cálculo:

$$
y=a * x,
$$

onde $x$ e $y$ são vetores e $a$ é escalar.

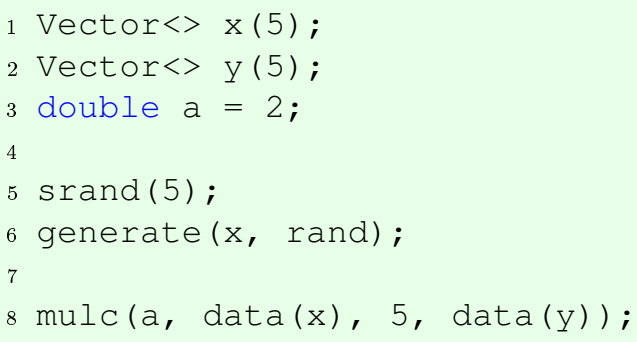

\section{mulc_add}

A função mulc_add faz o cálculo:

$$
z=s * x+y,
$$

onde $x, y$ e $z$ são vetores e $s$ é escalar.

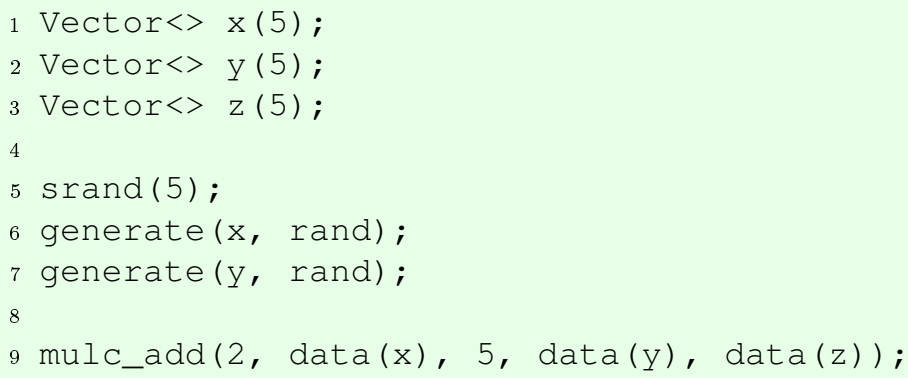

\section{mulc_add_iy}

A função mulc_add_iy faz o cálculo:

$$
y=a * x+y,
$$

onde $x$ e $x$ são vetores e $a$ é escalar.

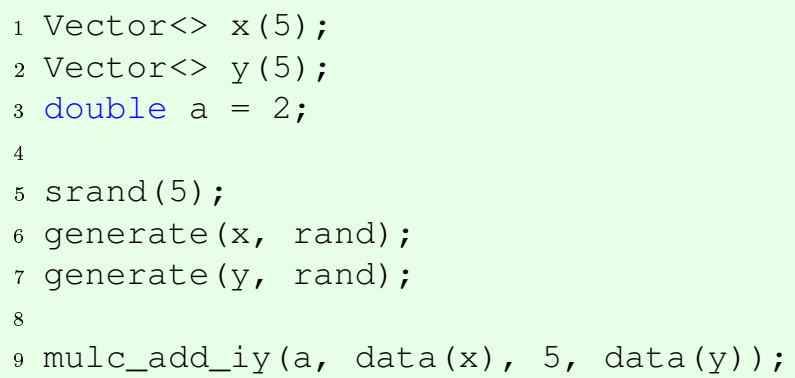


mulc_add_ix

A função mulc_add_ix faz o cálculo:

$$
x=a * x+y,
$$

onde $x$ e $y$ são vetores e $a$ é escalar.

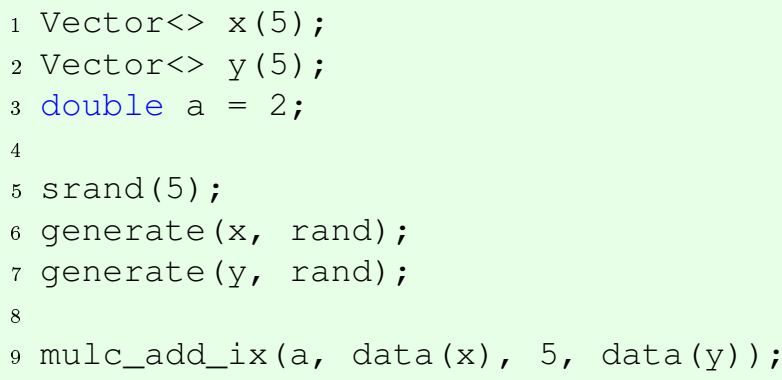

A função mulc_sub faz o cálculo:

$$
z=s * x-y
$$

onde $x, y$ e $z$ são vetores e $s$ é escalar.

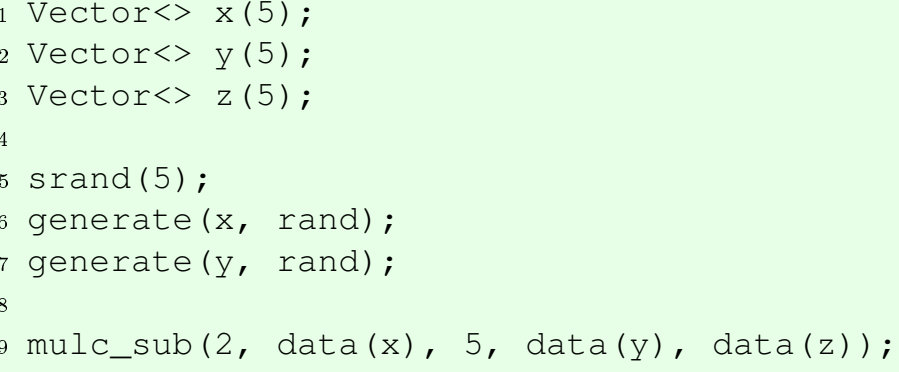

\subsubsection{Blas2}

matrix_x_vector

A função matrix_x_vector faz o cálculo:

$$
y=a * x
$$

onde $x$ e $y$ são vetores e $a$ é matrix.

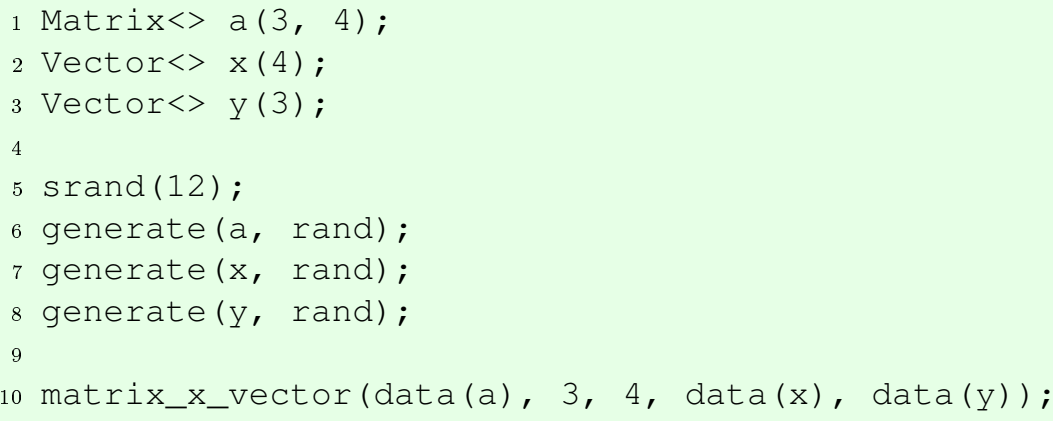

A função matrix_mul_vector_add faz o cálculo:

$$
z=a * x+y
$$

onde $x, y$ e $z$ são vetores e $a$ é matrix. 


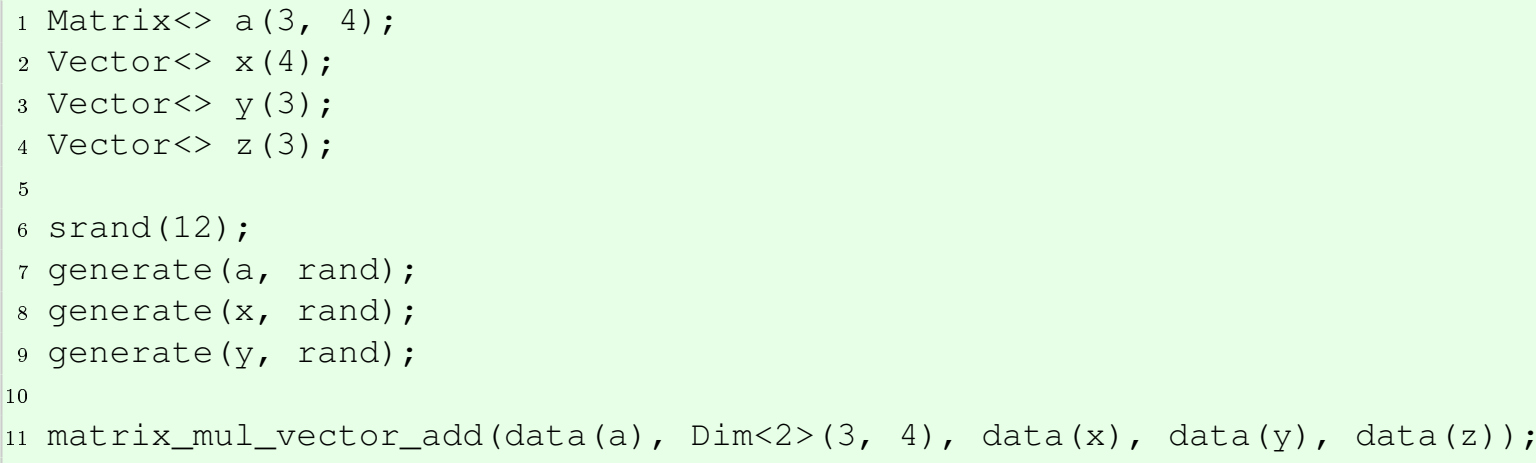

A função matrix_mul_vector_add_iy faz o cálculo:

$$
y+=a * x
$$

onde $x$ e $y$ são vetores e $a$ é matrix.

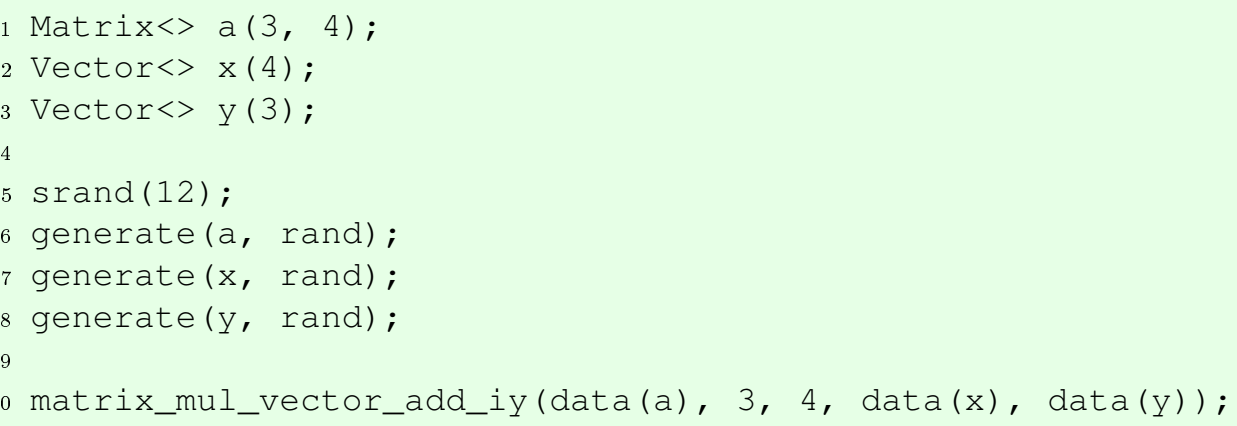

matrix_mul_vector_sub

A função matrix_mul_vector_sub faz o cálculo:

$$
z=a * x-y
$$

onde $x, y$ e $z$ são vetores e $a$ é matrix.

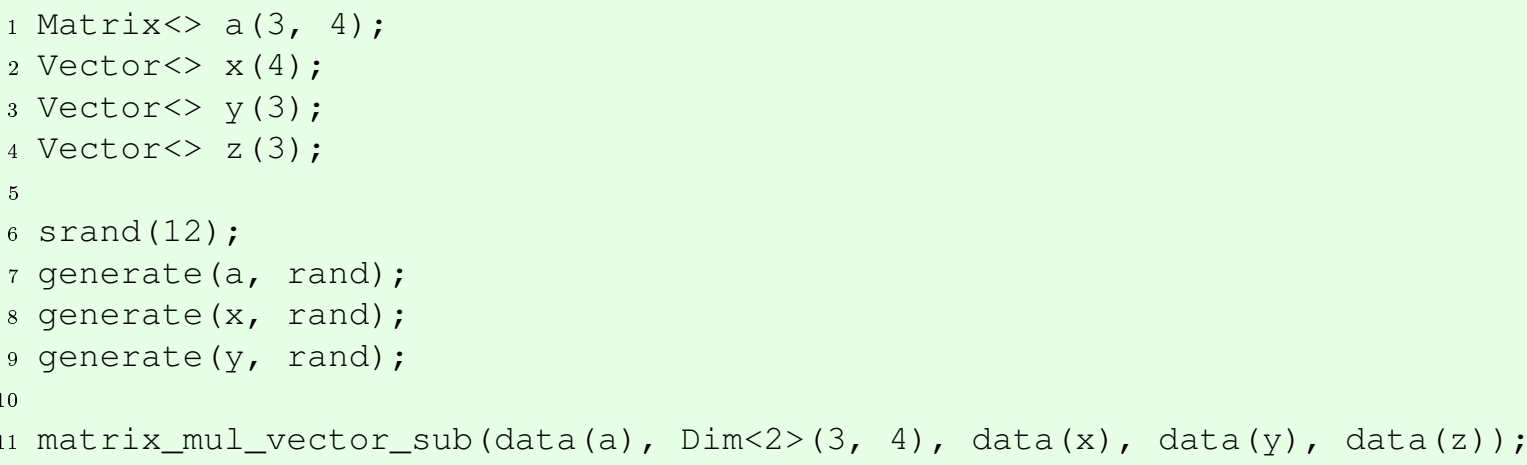

A função matrix_mul_vector_sub_iy faz o cálculo:

$$
y-=a * x
$$

onde $x$ e $y$ são vetores e $a$ é matrix. 


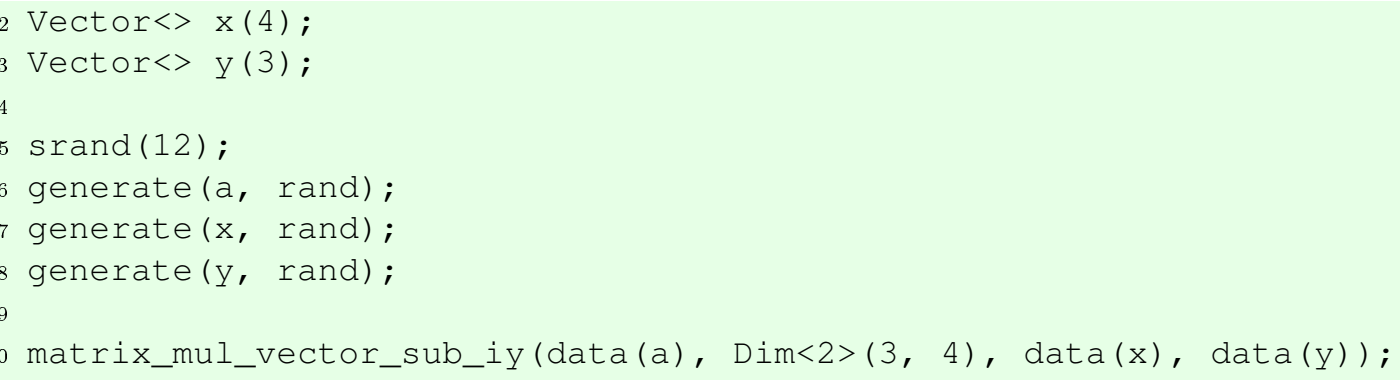

matrix_mul_vector_ rev_sub

A função matrix_mul_vector_rev_sub faz o cálculo:

$$
z=y-a * x
$$

onde $x, y$ e $z$ são vetores e $a$ é matrix.

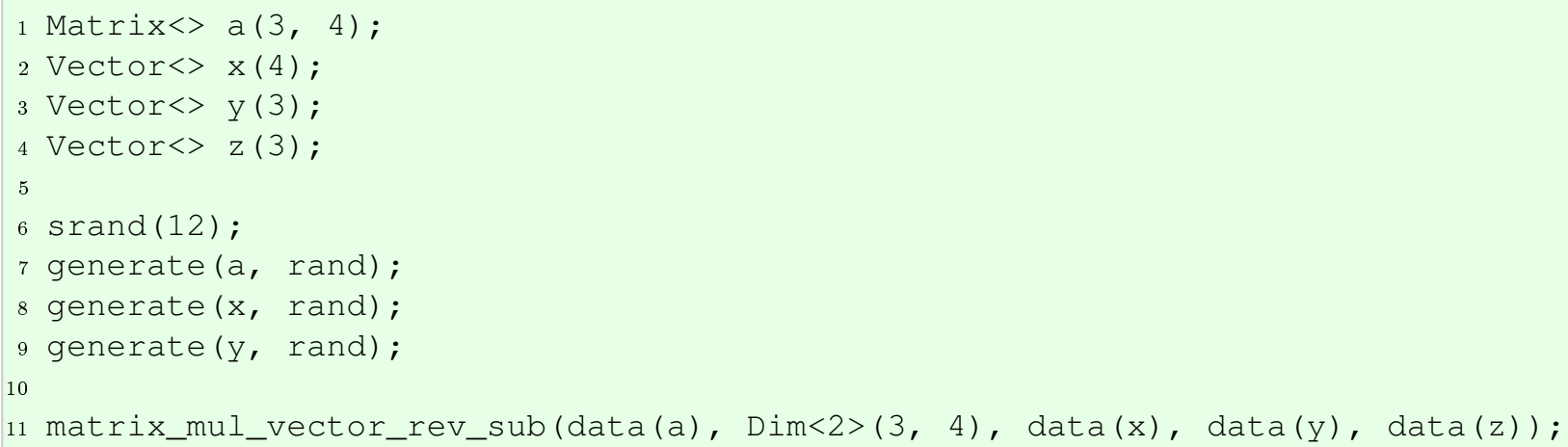

\section{transpose}

A função transpose faz o cálculo:

$$
b=a^{t}
$$

onde $b$ é a matriz transposta de $a$.

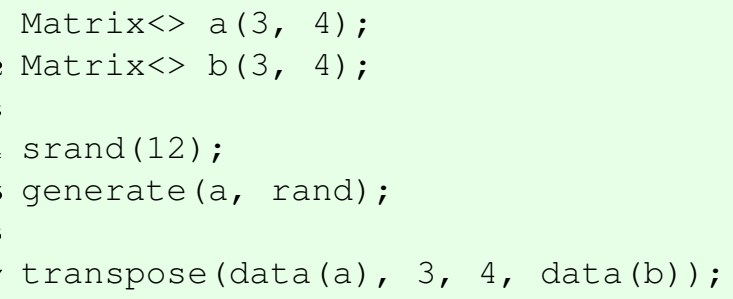

\subsubsection{Blas3}

\section{matrix $x_{-}$matrix}

A função matrix_matrix faz o cálculo:

$$
c=a * b,
$$

onde $a, b$ e $c$ são matrizes.

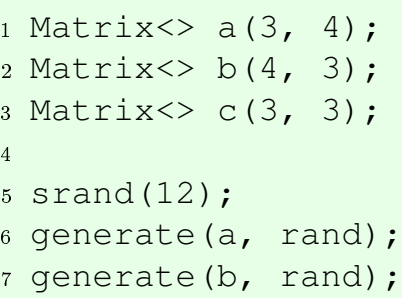


9 matrix_x_matrix(data(a), 3, 4, data(b), 3, data(c)) ;

matrix_mul_add

A função matrix_mul_add faz o cálculo:

$$
d=a * b+c,
$$

onde $a, b, c$ e $d$ são matrizes.

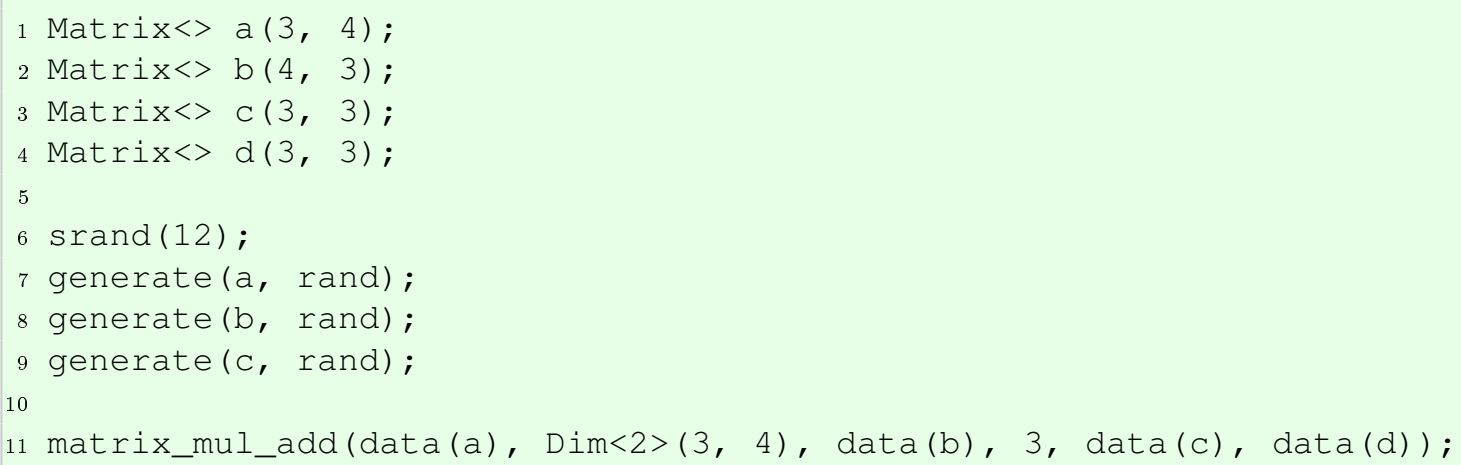

matrix_mul_add_ic

A função matrix_mul_add_ic faz o cálculo:

$$
c=a * b+c,
$$

onde $a, b$ e $c$ são matrizes.

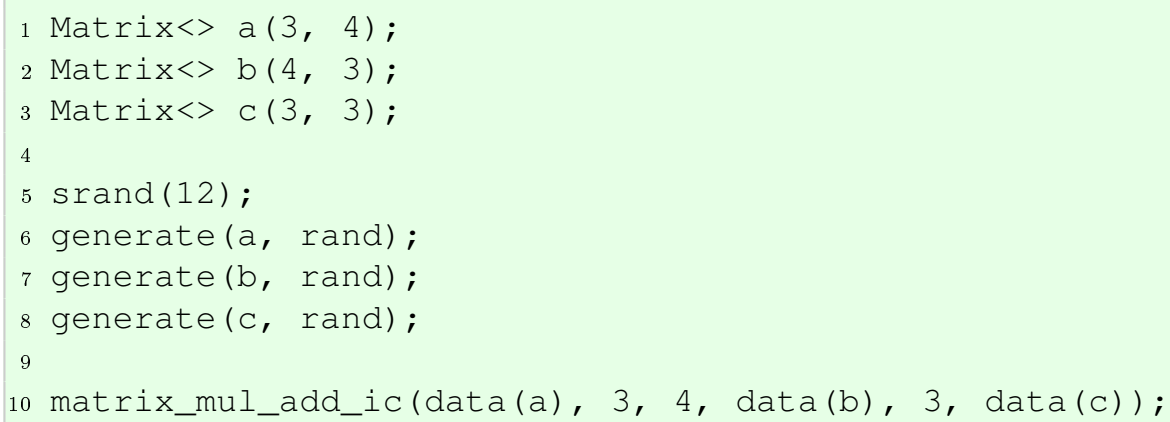

\section{matrix_mul_sub}

A função matrix_mul_sub faz o cálculo:

$$
d=a * b-c,
$$

onde $a, b, c$ e $d$ são matrizes.

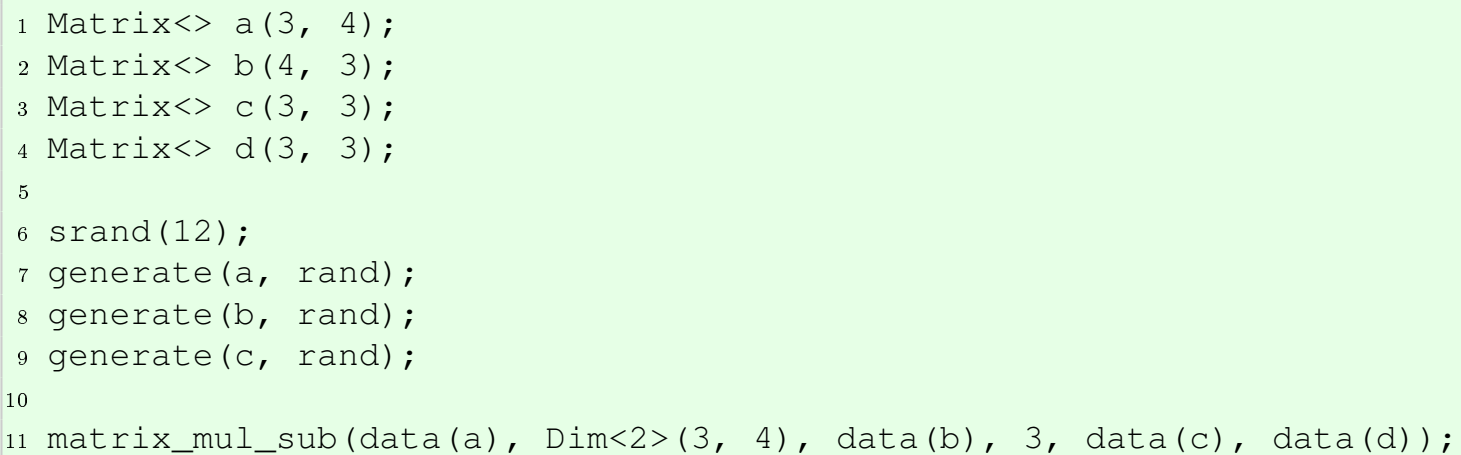


matrix_mul_sub_ic

A função matrix_mul_sub_ic faz o cálculo:

$$
c=a * b-c,
$$

onde $a, b$ e $c$ são matrizes.

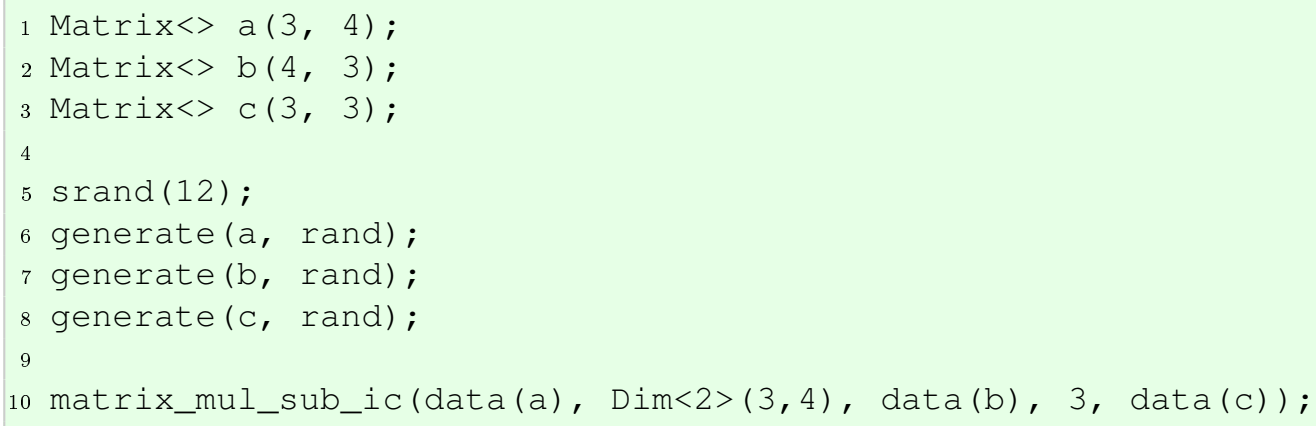

matrix_mul_rev_sub

A função matrix_mul_rev_sub faz o cálculo:

$$
d=c-a * b,
$$

onde $a, b, c$ e $d$ são matrizes.

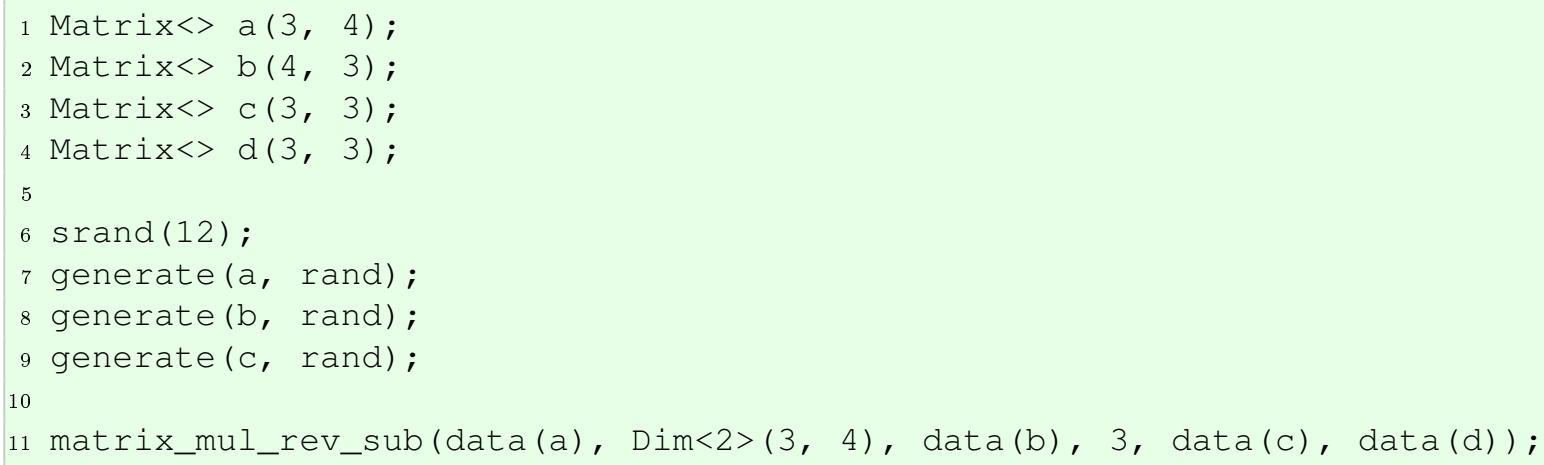

matrix_mul_rev_sub_ic

A função matrix_mul_rev_sub_ic faz o cálculo:

$$
c=c-a * b,
$$

onde $a, b$ e $c$ são matrizes.

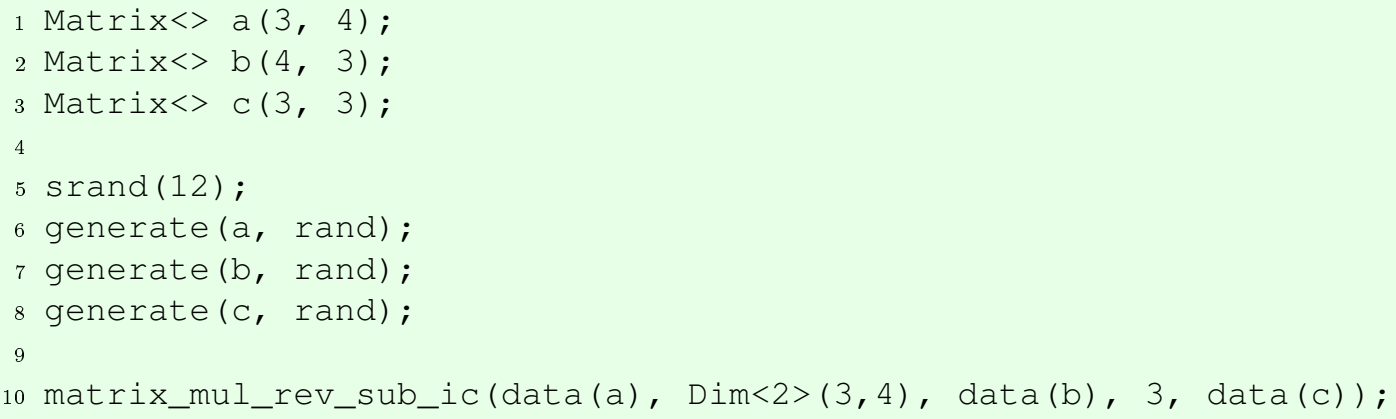




\section{matrix_x_transposed_matrix}

A função matrix_x_transposed_matrix faz o cálculo:

$$
p=a * b^{t},
$$

onde $a, b$ e $p$ são matrizes.

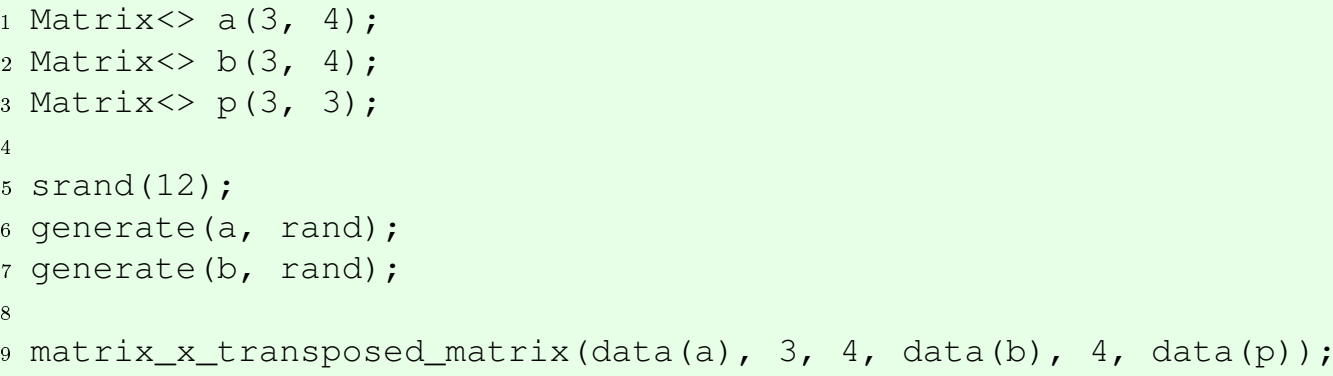

A função transposed_matrix_x_matrix faz o cálculo:

$$
p=a^{t} * b,
$$

onde $a, b$ e $p$ são matrizes.

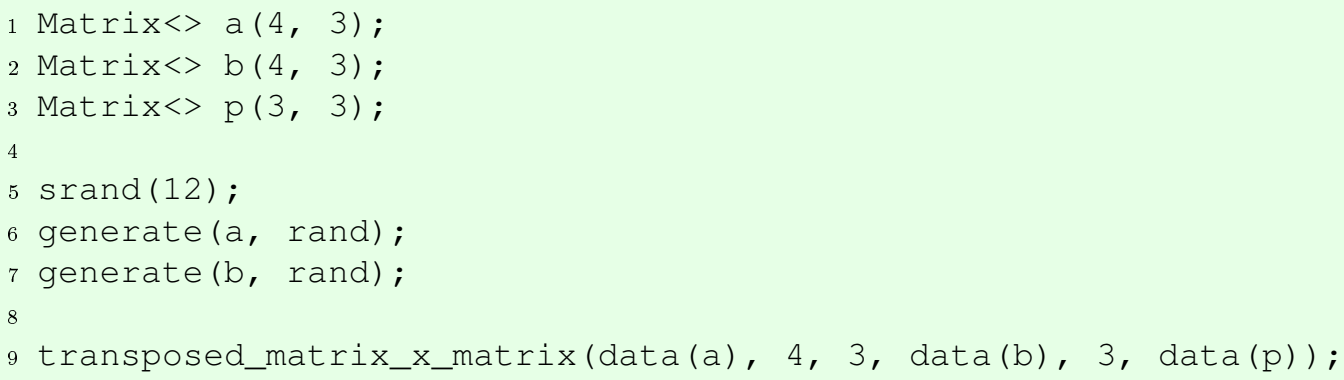




\section{Capítulo 6}

\section{Wrapper e estrutura de pastas da Klein}

\subsection{Wrapper}

Conforme visto no capítulo 4, é possível utilizarmos bibliotecas otimizadas para efetuar as operações da Klein. Isso é feito através da criação de wrapper com as funções desejadas. Nessa seção mostraremos como criar um wrapper para chamar as funções da biblioteca MKL.

A Klein define em tempo de compilação se utilizará a função default ou a da biblioteca otimizada, através da especialização de templates. Para facilitar, foi criada a pasta "../include/klein/blas/optimized" que é a pasta onde as especializações de templates para as bibliotecas serão implementadas.

\subsubsection{Blas1}

O arquivo "../include/klein/blas/blas1.h" contém as implementações default da biblioteca. Ele inclui o arquivo "../include/klein/blas/optimized/blas1_opt.h" que é onde podem ser implementadas as especializações de templates. Dessa forma, sempre que houver uma especialização de uma função, ela é que será chamada pela Klein.

\section{Soma de vetores}

A soma de vetores é realizada na Klein através da função template abaixo:

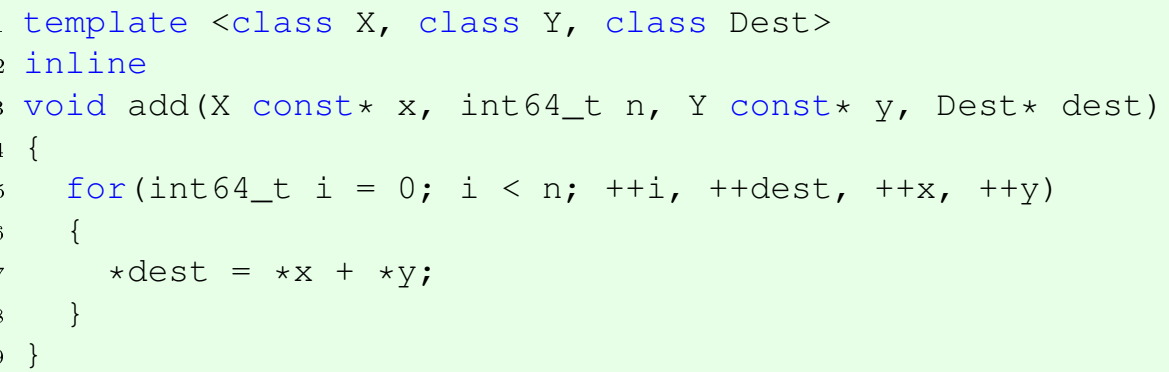

ela soma os elementos de $x$ e $y$ e armazena no vetor dest. Como trata-se de uma função genérica, ela sempre será chamada independente dos tipos contidos nos vetores (desde que haja as sobrecargas dos operadores soma e atribuição para os tipos envolvidos). Quando criamos a função abaixo:

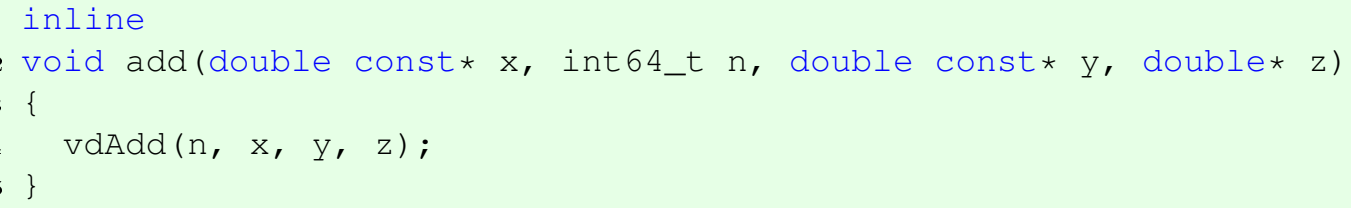

ela possui prioridade na chamada por se tratar de uma classe especializada. Portanto, quando fizermos a soma de dois vetores contendo o tipo double a Klein invocará a soma da MKL.

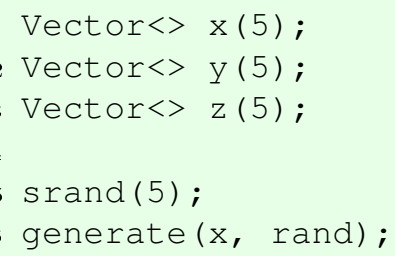




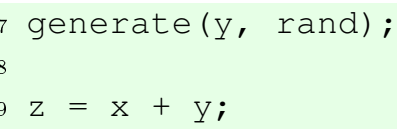

\subsubsection{Blas2}

O arquivo "../include/klein/blas/blas2.h" contém as implementações default da biblioteca. Ele inclui o arquivo "../include/klein/blas/optimized/blas2_opt.h" que é onde podem ser implementadas as especializações de templates.

\section{Multiplicação de matriz por vetores}

A multiplicação de matriz por vetor é realizada na Klein através da função template abaixo:

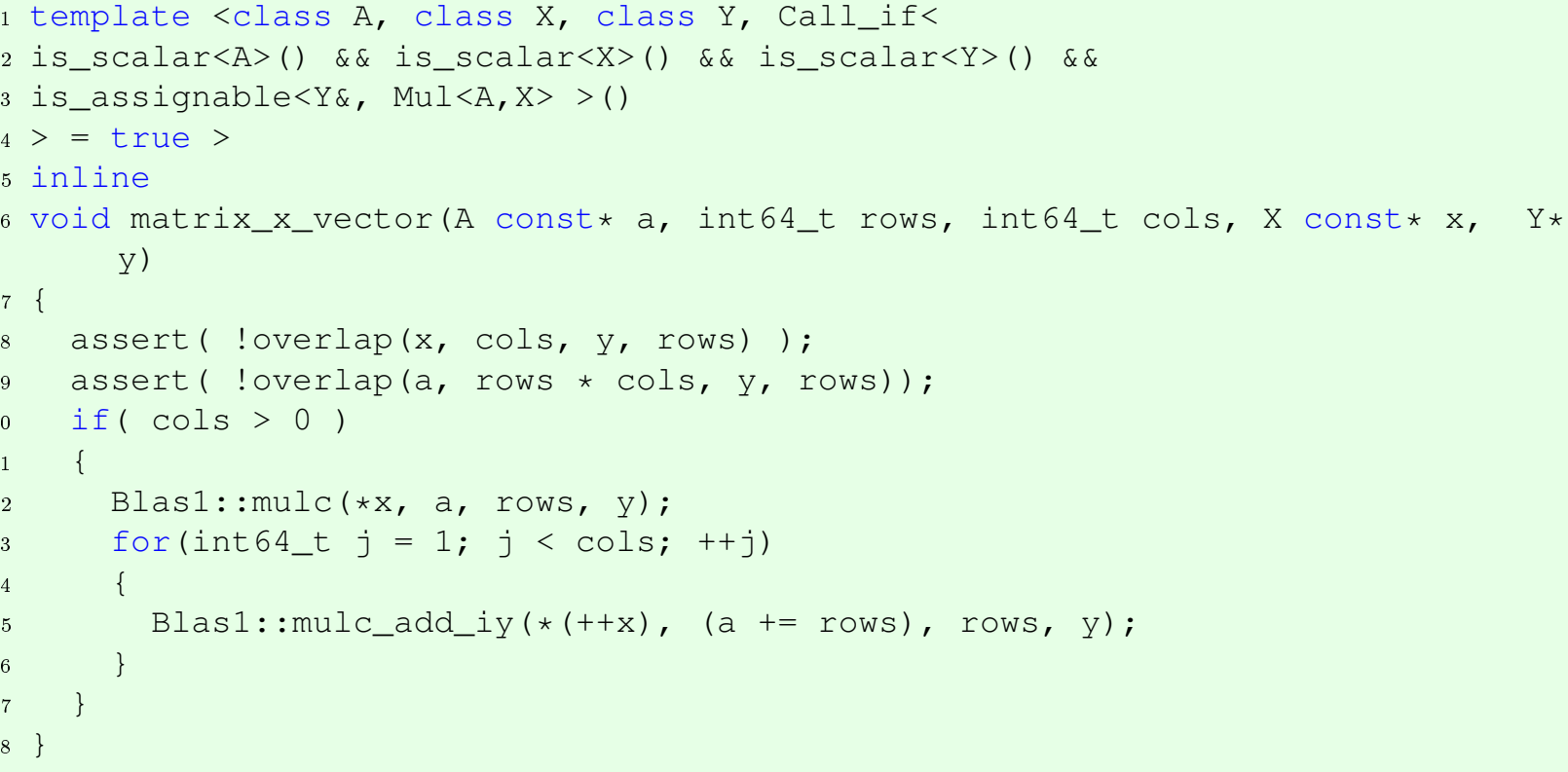

ela multiplica os elementos da matriz $a$ pelos elementos do vetor $x$ e armazena no vetor $y$.

Quando criamos a função abaixo:

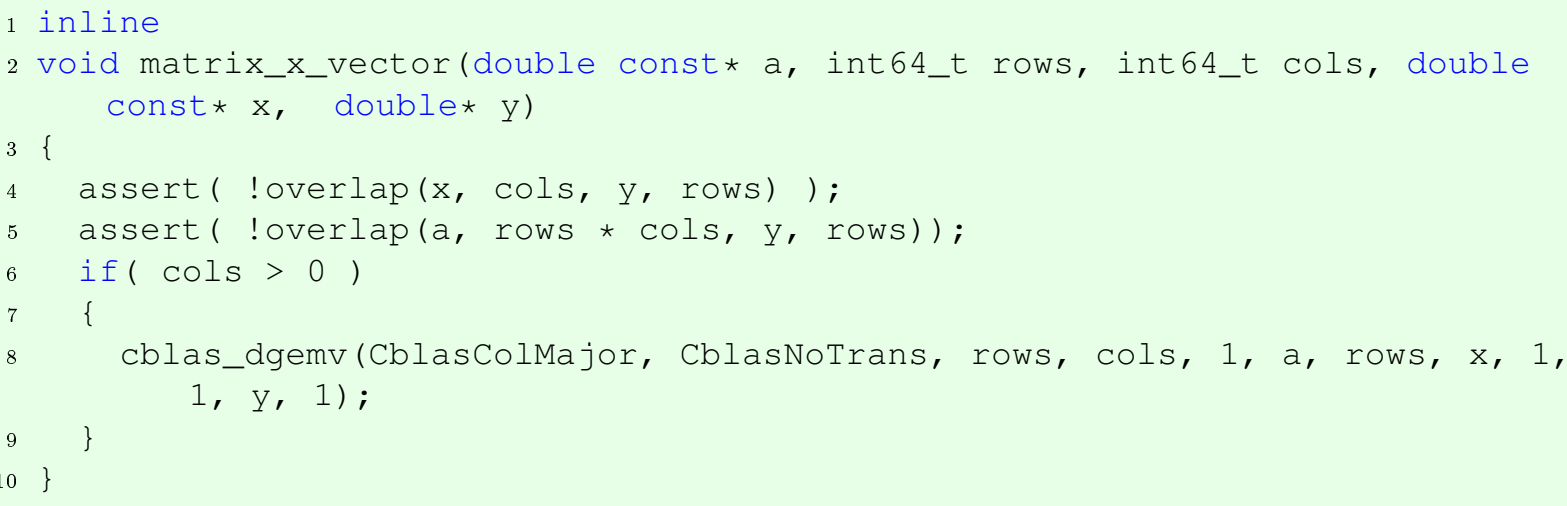

ela possui prioridade na chamada por se tratar de uma classe especializada. Portanto, quando fizermos a multiplicação de uma matriz por um vetor que contêm o tipo double a Klein invocará a multiplicação da MKL.

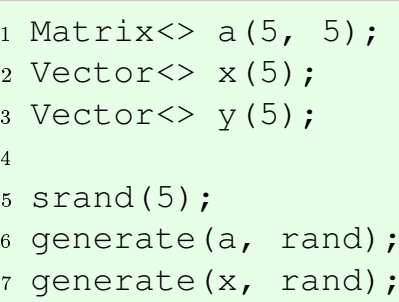




\section{Blas3}

O arquivo "../include/klein/blas/blas3.h" contém as implementações default da biblioteca. Ele inclui o arquivo "../include/klein/blas/optimized/blas3_opt.h" que é onde podem ser implementadas as especializações de templates.

\section{Multiplicação de matrizes}

A multiplicação de matrizes é realizada na Klein através da função template abaixo:

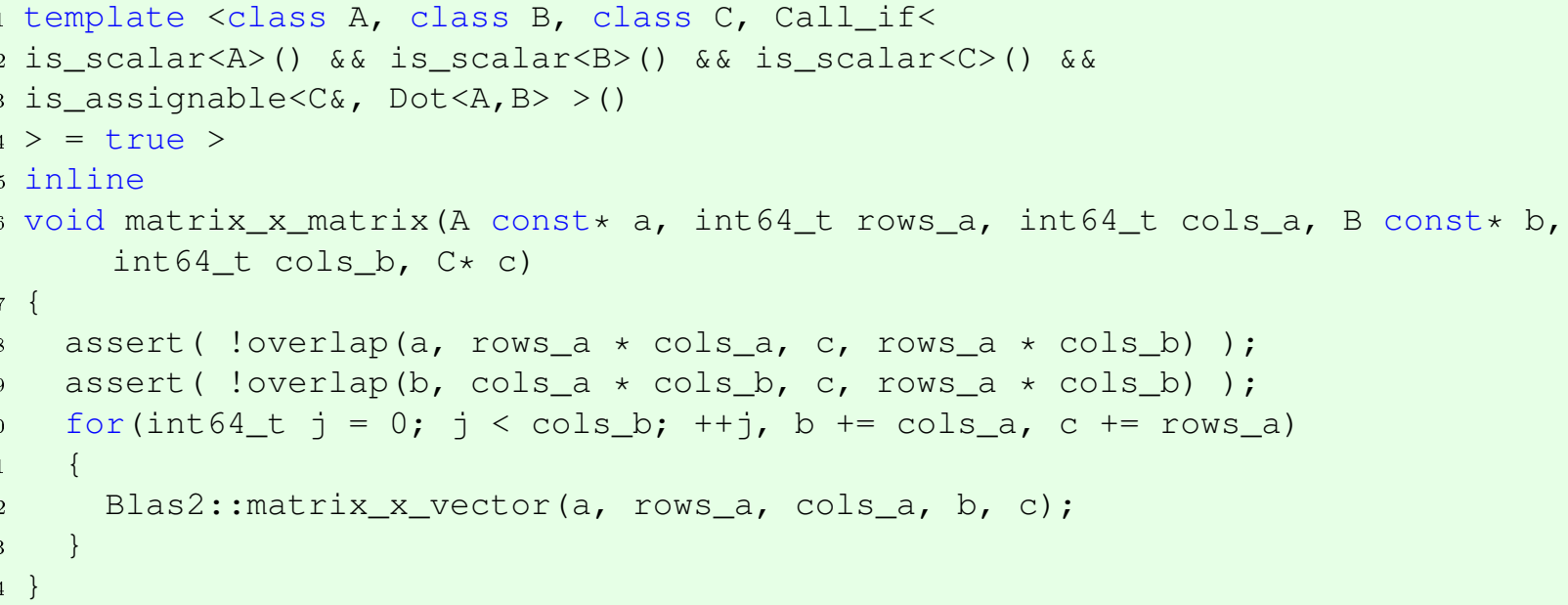

ela multiplica os elementos da matriz $a$ pelos elementos da matriz $b$ e armazena o resultado na matriz $c$.

Quando criamos a função abaixo:

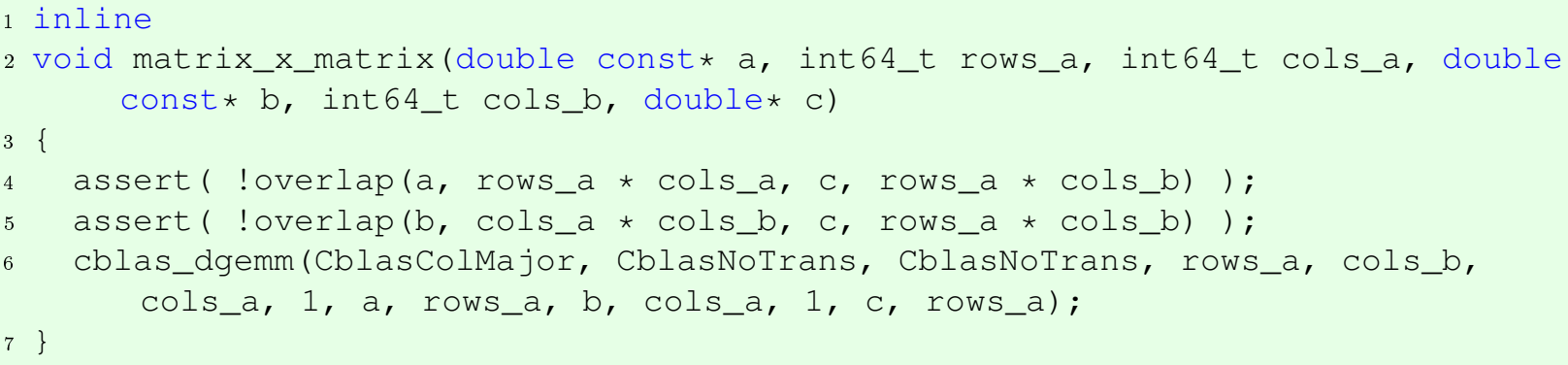

ela possui prioridade na chamada por se tratar de uma classe especializada. Portanto, quando fizermos a multiplicação de duas matrizes que contêm o tipo double a Klein invocará a multiplicação da MKL.

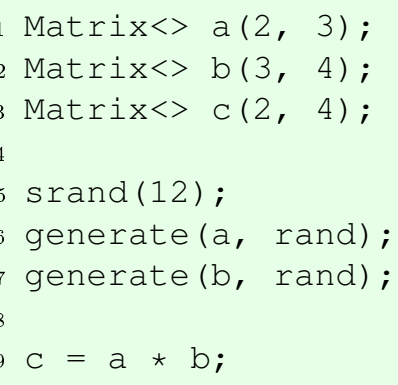

\subsection{Estrutura de pastas}

A Klein está organizada em pastas, cuja hierarquia está disposta desta forma:

- klein: pasta raiz. 
- blas: contém os arquivos referentes às funções básicas de álgebra linear.

- factorizations: contém os arquivos referentes às funções de fatoração de matrizes.

- interpolation: contém os arquivos referentes às funções de interpolação.(lagrange, barycentric, chebyshev)

- intervals: contém os arquivos referentes às funções de aritmética intervalar.

- io: contém os arquivos de leitura e escrita de dados.

- lazy: contém os arquivos referentes às sobrecargas dos operadores para implementação da estratégia lazy na Klein.

- linear_maps: contém os arquivos referentes às funções dos operadores lineares.

- memory: contém os arquivos referentes às funções de alocação de memória, inclusive de forma alinhada.

- mpl: contém os arquivos referentes às funções de tratamento de tipos. As funções da Klein impedem que os tipos genéricos passados em uma função sejam incompatíveis.

- optimization: contém os arquivos referentes às funções de programação linear.

- combinatorics: contém os arquivos referentes às funções cálculos combinatórios.

- scalars: contém os arquivos referentes aos tipos numéricos implementados na Klein.

- vectors: contém os arquivos referentes ao vetor cujas entradas são escaladas por um mesmo expoente. 


\section{Capítulo 7}

\section{Teste de Software}

Testes são fundamentais no desenvolvimento de software. Porém não basta utilizarmos uma grande quantidade de testes, é necessário que eles sejam bem formulados. Para isto, é imprescindível que esta etapa seja planejada e esteja presente no cronograma do projeto. Para que o planejamento seja eficaz, devemos conhecer as boas práticas adotadas para este fim. O conhecimento destas técnicas proporcionará agilidade e qualidade na geração e execução dos testes. Basicamente, as técnicas de teste são divididas em caixa preta e caixa branca [6], conforme descritas abaixo.

\subsection{Caixa preta}

As técnicas caixa preta geram os chamados testes funcionais. Elas não levam em conta o código fonte na criação dos testes. Estas técnicas geram os testes através do estudo das especificações do software e a identificação das entradas e saídas possíveis.

Em um mundo ideal, a combinação de todas as entradas da aplicação bastaria para garantir que o software não contém erro. Porém na maioria das vezes isto é inviável devido a grande quantidade de combinações. Uma abordagem mais realista é a subdivisão dos valores de entrada no menor número de subconjuntos possíveis, tais que o teste de qualquer elemento de um subconjunto seria equivalente a testá-lo por inteiro. Estratégias que fazem parte desta técnica são: Particionamento em Classes de Equivalência e Análise de Valor Limite.

\subsubsection{Particionamento em classes de equivalência}

A técnica de Particionamento em Classes de Equivalência consiste na divisão do domínio de entrada da aplicação em classes de dados. Cada classe possui um conjunto de elementos cujos comportamentos são idênticos ao adentrarem no sistema. Desta forma, basta a execução do teste de um dos elementos de uma classe, para que esta esteja completamente testada.

O particionamento do domínio deve considerar o comportamento do sistema para cada valor de entrada. Assim, se um elemento de uma classe detecta um erro, este repercutirá em todos os elementos daquela classe. Seguem alguns casos de particionamento:

- Se uma condição de entrada do programa especifica o intervalo de valores $[x, y]$. Então teremos uma classe que representará os valores válidos, que consistem dos valores entre $x$ e $y$. E outras duas que representarão os valores inválidos, que consistem dos valores menores que $x$ e maiores que $y$.

- Se uma condição de entrada delimita a quantidade de valores passados como parâmetro. Por exemplo, caso esta condição fosse um conjunto de 1 a 3 itens, as classes de equivalência poderiam ser divididas em três. Uma classe de equivalência válida, que consiste da quantidade de entrada entre 1 e 3 . E duas inválidas, que consistem de "nenhum item" e a "quantidade de itens maior que $3 "$

- Se uma condição de entrada especifica, para um conjunto de valores, tratamentos diferentes pelo programa. Por exemplo, caso as entradas a e b fossem tratadas de forma exclusiva, haveria uma classe para a entrada a, outra classe para a entrada b e uma terceira que representaria as outras entradas do sistema. 
Após definir as classes de equivalência para cada condição de entrada, são gerados os casos de teste. Cada classe deve ser verificada individualmente.

\subsubsection{Análise de valor limite}

A técnica Análise de Valor Limite utiliza para testes os valores da fronteira do domínio de entrada. Muitos erros tendem a ocorrer quando utilizamos estes valores. Normalmente quando esta técnica é usada, os valores imediatamente acima e abaixo dos limites superior e inferior são testados. Seguem alguns casos onde é aplicável:

- Se uma condição de entrada especifica uma faixa de valores, devemos definir casos de teste para seus extremos e os valores imediatamente acima e abaixo dos extremos. Por exemplo, caso a condição seja os valores inteiros entre 4 e 12. Então os valores que teríamos seriam 3 , $4,5,11,12$ e 13 .

- Se uma condição de entrada delimita a quantidade de valores passados como parâmetro. Por exemplo, caso esta condição fosse um conjunto de 1 a 34 itens, deveríamos testar "nenhum item", "1 item", "2 itens", "33 itens", "34 itens" e "35 itens".

Tanto a técnica de Particionamento em Classes de equivalência, como a técnica de Análise de Valor Limite exigem a divisão da entrada do programa, porém a primeira explora as situações nos extremos das partições.

\subsection{Caixa Branca}

As técnicas caixa branca, que geram os chamados testes estruturais, são aquelas onde o código fonte é levado em conta na criação dos testes. Estas técnicas geram os testes através do estudo do código fonte, identificando internamente as condições e os fluxos dos dados da aplicação. Dentre os critérios desta técnica temos: Cobertura por Decisão, Cobertura por Condição, Cobertura por Decisão e Condição e Cobertura por Múltiplas Condições. Para exemplificar cada um destes critérios utilizaremos o código abaixo:

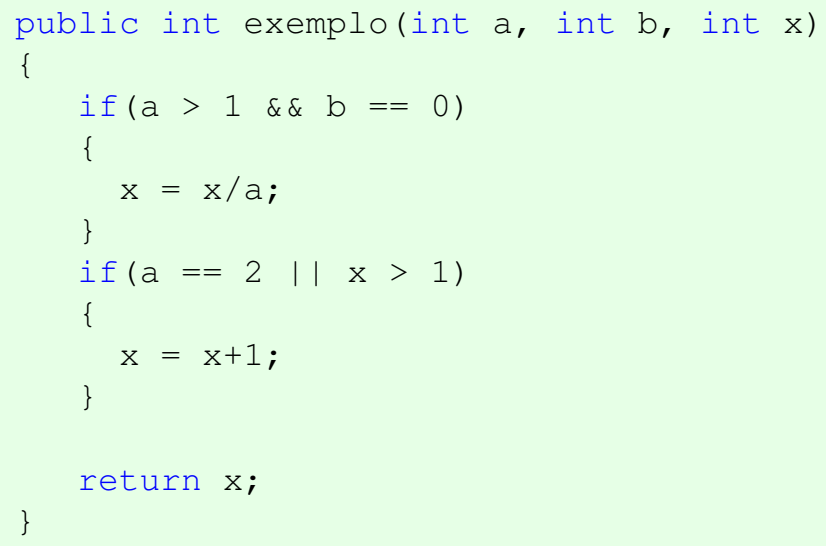

\subsubsection{Cobertura por Decisão}

Neste critério devem ser escritos casos de testes suficientes para que se obtenha de cada decisão um retorno verdadeiro e um falso. Em outras palavras, todas as direções de uma decisão devem ser tomadas ao menos uma vez. Por exemplo, podemos utilizar na função exemplo os valores de entrada:

- $\mathrm{a}=3, \mathrm{~b}=0$ e $\mathrm{x}=1$

- $\mathrm{a}=2, \mathrm{~b}=1$ e $\mathrm{x}=3$

Porém logo identificamos uma fraqueza deste critério, onde problemas na condição de $\mathrm{x}$ não seriam identificados. Caso ao invés de $\mathrm{x}>1$ tivéssemos colocado erroneamente $\mathrm{x}<1$, os testes teriam passado e não teríamos encontrado o erro. 


\subsubsection{Cobertura por Condição}

Para suprir algumas fraquezas da cobertura por decisão, existe o critério de cobertura por condição. São escritos casos de testes suficientes para que todos os retornos das condições de uma decisão sejam obtidas ao menos uma vez. Por exemplo, na função exemplo temos as seguintes condições $\mathrm{a}>1, \mathrm{~b}=0, \mathrm{a}=2$ e $\mathrm{x}>1$. Portanto, precisamos de entradas que satisfaçam $\mathrm{a}>1, \mathrm{a}<=1$, $\mathrm{b}=0, \mathrm{~b}<>0, \mathrm{a}=2, \mathrm{a}<>2$ e $\mathrm{x}>1$ e $\mathrm{x}<=1$. Para isto, podemos utilizar como entrada os valores:

- $\mathrm{a}=2, \mathrm{~b}=1$ e $\mathrm{x}=4$

- $\mathrm{a}=1, \mathrm{~b}=0$ e $\mathrm{x}=1$

Como temos uma decisão do tipo if $(X$ and $Y$ ), corremos o risco de definir testes que resultem no contrário para diferentes condições de uma mesma decisão. Ou seja, $\mathrm{X}$ pode ser verdadeiro quando Y é falso e vice-versa. Assim, o código executado quando a decisão é verdadeira não teria sido testado. Isto ocorre, nas entradas que definimos com as variáveis a e b.

\subsubsection{Cobertura por Decisão e Condição}

Neste critério devem ser escritos casos de testes suficientes para que todos os retornos das decisões e das condições de uma decisão sejam obtidas, e todos os pontos da função sejam executados ao menos uma vez. Para exemplificar utilizamos as entradas:

- $\mathrm{a}=2, \mathrm{~b}=0$ e $\mathrm{x}=4$

- $\mathrm{a}=1, \mathrm{~b}=1$ e $\mathrm{x}=1$

A fraqueza deste critério está nos resultados das condições and e or, que podem mascarar a evolução de outras condições. Ou seja, podemos ter casos em que uma decisão é satisfeita devido a uma única condição, sendo as outras nunca verificadas. Isto ocorre com a variável x na primeira entrada. $\mathrm{O}$ fato de a condição a $=2$ retornar verdadeiro implica na não verificação da condição $\mathrm{x}>1$. Com estas deduções concluímos que se a condição estivesse escrita erroneamente como if $(a==2$ // $x==1$ ), não teríamos notado o erro com as entradas que utilizamos.

\subsubsection{Cobertura por Múltiplas Condições}

Este critério requer que escrevamos casos de testes suficientes que retornem todas as combinações de condições de uma decisão e que cada ponto do código seja executado ao menos uma vez. Reorganizando as linhas de código da função exemplo para melhor entendermos este critério:

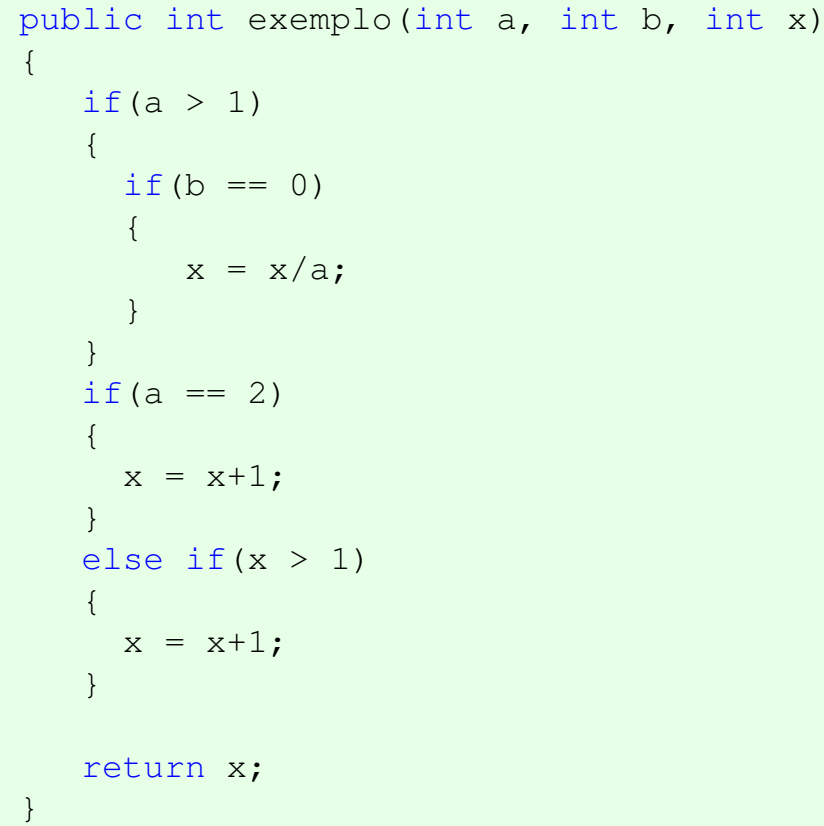


A partir deste código fica mais claro as condições que devemos testar. Abaixo as combinações de condições que devemos cobrir:

- $\mathrm{a}>1, \mathrm{~b}=0$

- $\mathrm{a}>1, \mathrm{~b}<>0$

- $\mathrm{a}<=1, \mathrm{~b}=0$

- $\mathrm{a}<=1, \mathrm{~b}<>0$

- $\mathrm{a}=2, \mathrm{x}>1$

- $\mathrm{a}=2, \mathrm{x}<=1$

- $\mathrm{a}<>2, \mathrm{x}>1$

- $\mathrm{a}<>2, \mathrm{x}<=1$

Apesar de oito combinações, isto não implica que devemos efetuar oito casos de teste. No exemplo acima este critério pode ser coberto através dos seguintes casos de teste:

- $\mathrm{a}=2, \mathrm{~b}=0, \mathrm{x}=4$ (cobre as combinações 1 e 5 )

- $\mathrm{a}=2, \mathrm{~b}=1, \mathrm{x}=1$ (cobre as combinações 2 e 6 )

- $\mathrm{a}=1, \mathrm{~b}=0, \mathrm{x}=2$ (cobre as combinações 3 e 7 )

- $\mathrm{a}=1, \mathrm{~b}=1, \mathrm{x}=1$ (cobre as combinações 4 e 8 )

\subsection{Etapas de teste}

As etapas de testes podem ser divididas em Teste de Unidade, Teste de Integração, Teste de Sistema e Teste de Aceitação.

\subsubsection{Teste de Unidade}

Nesta etapa, cada unidade da aplicação é testada individualmente. Uma unidade é a menor parte testável do código, que pode ser uma rotina ou um procedimento. O objetivo é a identificação de erros em pequenas partes independentes do código.

Os testes unitários geralmente são executados pelo desenvolvedor, sendo então considerado parte da implementação. E devido a isto, o que se predomina nesta etapa é a utilização de técnicas caixa branca.

\subsubsection{Teste de Integração}

Nesta etapa, são testadas as interrelações entre as unidades da aplicação. Apenas a integração entre as unidade são levadas em conta, não havendo foco nas unidades individualmente. O objetivo desta etapa é encontrar falhas na integração entre os componentes do sistema.

Como os testes integrados geralmente são executados pelo desenvolvedor, o mais comum é a utilização de técnicas caixa branca. Geralmente os erros identificados são de transmissão de dados, podendo haver informações incompletas ou incorretas. É essencial que nesta etapa o código já tenha passado pelos testes unitários e funcionado.

\subsubsection{Teste de Sistema}

Os testes de sistema são preferencialmente executados em um ambiente similar ao de produção. Através do ponto de vista do usuário, é verificado se as especificações estão todas implementadas. O objetivo desta etapa é simular o ambiente real da aplicação.

Nesta etapa, espera-se que os testes de integração tenham rodado com sucesso e o sistema esteja pronto para ser testado por uma equipe que desconheça seus aspectos técnicos. Portanto, são utilizadas técnicas caixa preta. Testes de carga, desempenho e confiabilidade também são executados nesta etapa. 


\subsubsection{Teste de Aceitação}

Os testes de aceitação, assim como os testes de sistema, são preferencialmente executados em um ambiente similar ao de produção. Neste etapa são comparados o programa com os requisitos iniciais do programa. O objetivo é verificar se o produto desenvolvido satisfaz as necessidades do cliente.

Além disso, podem ser efetuados testes de desempenho, segurança, recuperação de falhas, configurações e testes funcionais. Esta etapa é executada pelo usuário final e a aprovação dos testes depende dele. 



\section{Capítulo 8}

\section{Testes de desempenho}

Já vimos que a manipulação de vetores e matrizes é feita de forma intuitiva pela Klein. Neste capítulo, nosso objetivo é medir o tempo de execução de alguns algoritmos e bibliotecas para identificar possíveis melhorias na Klein e mostrar que, apesar de ser uma biblioteca parametrizável, possui um bom desempenho. Vamos compará-lo com as bibliotecas MKL e ATLAS, além de algoritmos que se utilizam da técnica expression template[13].

A MKL (Intel Math Kernel Library)[9] foi criada para otimizar rotinas matemáticas básicas. Ela é otimizada para os processadores Intel, incluindo processadores com múltiplos cores. A MKL oferece rotinas de álgebra linear, transformada de Fourier, funções matemáticas vetorizadas e funções geradoras de números aleatórios.

O projeto ATLAS (Automatically Tuned Linear Algebra Software)[3] é um estudo contínuo focado em aplicar técnicas empíricas para proporcionar melhor desempenho em aplicações de álgebra linear. No momento ela provê interface para uma implementação eficiente da BLAS.

Os testes abaixo foram rodados em uma máquina com o processador Intel $($ Xeon(R) E5-2640 v2 @ 2.00GHz x 17, 64GB de RAM, sistema operacional Ubuntu 13.04 e utilizando o compilador $\operatorname{gcc} 4.8 .1$.

\subsection{Soma de vetores}

A primeira comparação de tempo que fizemos foi para soma de vetores. Criamos funções que calculam as expressões:

$$
\begin{gathered}
r e s p=a+b, \\
r e s p=a+b+c, \\
r e s p=a+b+c+d, \\
r e s p=a+b+c+d+e, \\
r e s p=a+b+c+d+e+f, \\
r e s p=a+b+c+d+e+f+g, \\
r e s p=a+b+c+d+e+f+g+h, \\
r e s p=a+b+c+d+e+f+g+h+i, \\
r e s p=a+b+c+d+e+f+g+h+i+j,
\end{gathered}
$$

onde resp, $a, b, c, d, e, f, g, h, i$ e $j$ são vetores, utilizando as estratégias expression template e dummy, e as bibliotecas Klein, MKL e ATLAS.

Abaixo mostramos como ficam os códigos dessas bibliotecas para a expressão resp $=a+b+$ $c+d+e+f+g+h+i+j$. O código para as bibliotecas MKL e ATLAS é o mesmo e fica assim:

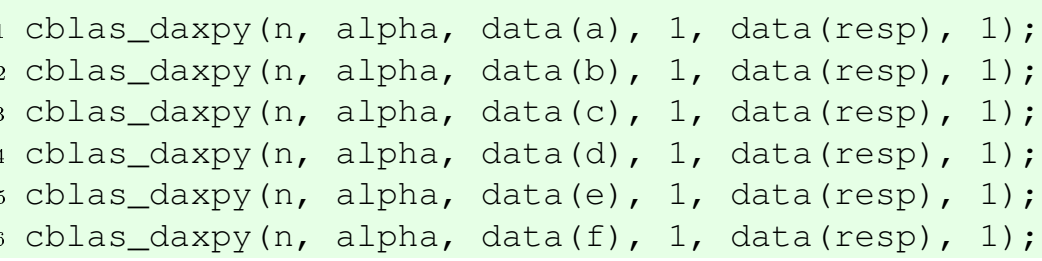




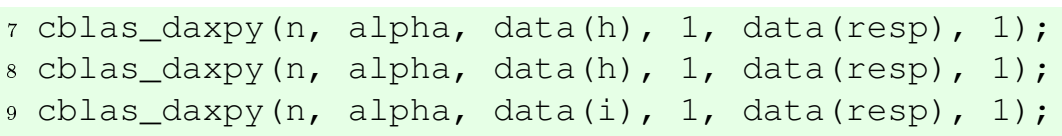

O código para a estratégia expression template fica dessa maneira:

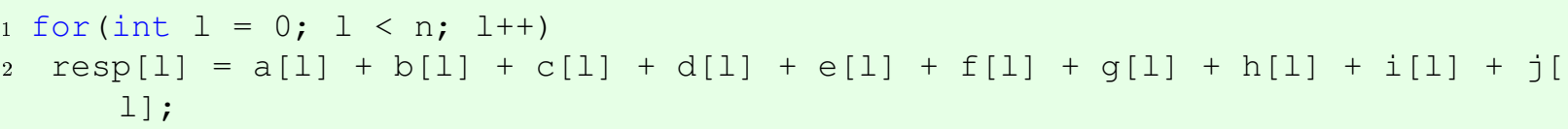

O código dummy fica dessa outra forma:

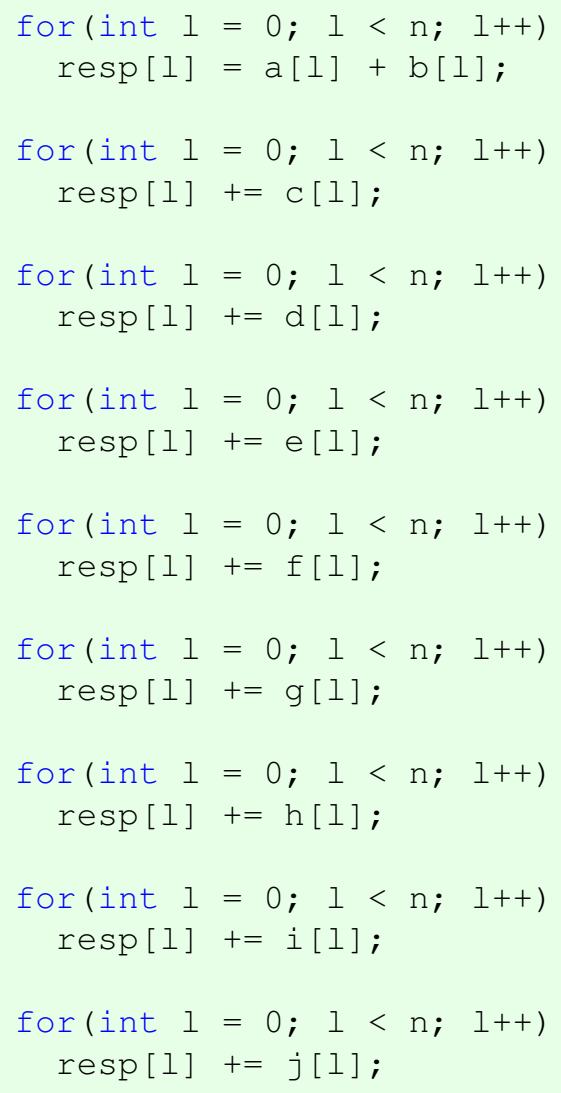

E o código da Klein, dessa maneira:

$1 \operatorname{resp}=a+b+c+d+e+f+g+h+i+j ;$

Nesse caso, não há dúvida do quanto o código da Klein é mais simples e fácil de entender. Mas agora estamos interessados em medir o desempenho desse código perante os demais.

A primeira implementação de soma de vetores da Klein efetuava a soma dos primeiros vetores e armazenava no elemento destino. Posteriormente, somava o terceiro elemento no destino, de forma inplace, e assim por diante. Tendo como motivação os tempos dos algoritmos da seção Estratégias lazy, decidimos utilizar a estratégia expression template para soma de vetores e soma de matrizes na Klein. Essa implementação gerou os seguintes resultados de comparação de tempo:

\section{Atlas / Klein}

Quando comparamos os tempos da biblioteca ATLAS com a Klein notamos que para vetores a partir de 100 elementos a Klein possui um desempenho superior na maioria dos casos e competitivo nos outros. 


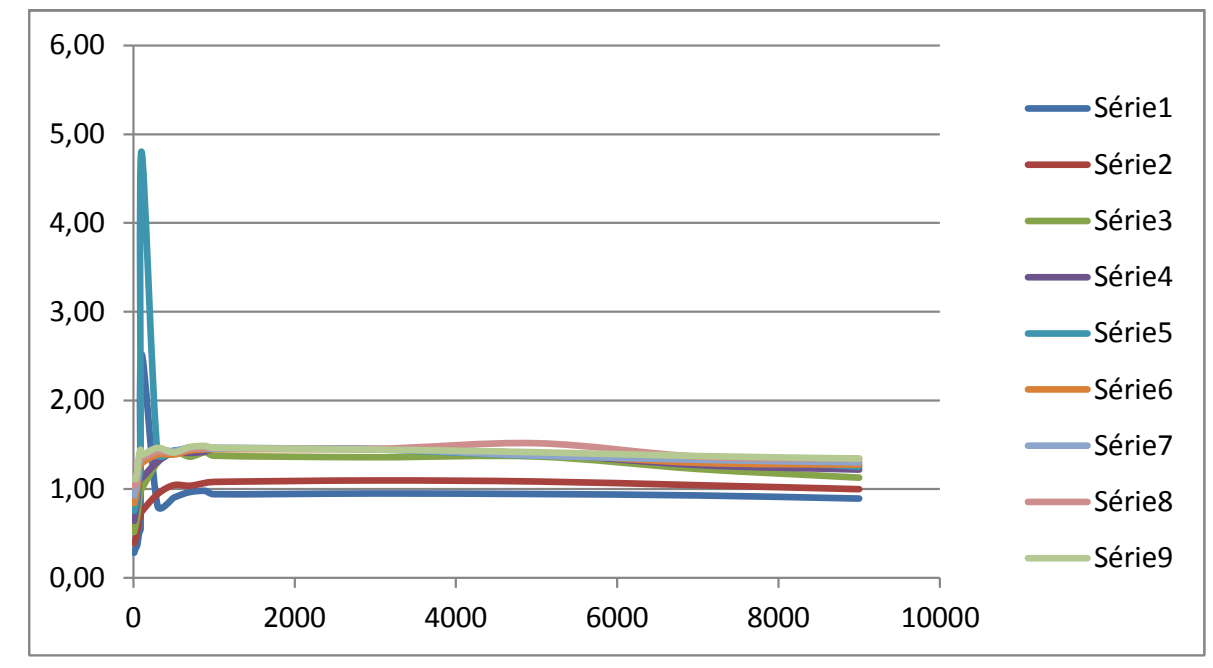

Figura 8.1: Soma vetores - Atlas / Klein

Quando comparamos os tempos utilizando vetores pequenos, entre 10 e 90 elementos, notamos que a medida que aumentamos o número de vetores sendo somados em conjunto com o número de elementos dos vetores a Klein começa a ficar mais rápida.

\begin{tabular}{|c|c|c|c|c|c|}
\hline $\begin{array}{c}\text { Tempo Atlas } \\
\text { Tempo Klein }\end{array}$ & \multicolumn{5}{|c|}{ Número de elementos } \\
\hline Função & $\mathbf{1 0}$ & $\mathbf{3 0}$ & $\mathbf{5 0}$ & $\mathbf{7 0}$ & $\mathbf{9 0}$ \\
\hline $\mathbf{8 . 1}$ & 0,28 & 0,34 & 0,37 & 0,51 & 0,56 \\
\hline $\mathbf{8 . 2}$ & 0,39 & 0,45 & 0,55 & 0,68 & 0,68 \\
\hline $\mathbf{8 . 3}$ & 0,52 & 0,61 & 0,70 & 0,87 & 0,99 \\
\hline $\mathbf{8 . 4}$ & 0,64 & 0,76 & 0,88 & $\mathbf{1 , 0 8}$ & $\mathbf{1 , 1 7}$ \\
\hline $\mathbf{8 . 5}$ & 0,75 & 0,83 & 0,96 & $\mathbf{1 , 1 3}$ & $\mathbf{1 , 2 4}$ \\
\hline $\mathbf{8 . 6}$ & 0,84 & 0,93 & $\mathbf{1 , 0 1}$ & $\mathbf{1 , 2 2}$ & $\mathbf{1 , 2 8}$ \\
\hline $\mathbf{8 . 7}$ & 0,93 & $\mathbf{1 , 0 2}$ & $\mathbf{1 , 1 0}$ & $\mathbf{1 , 2 8}$ & $\mathbf{1 , 3 5}$ \\
\hline $\mathbf{8 . 8}$ & $\mathbf{1 , 0 4}$ & $\mathbf{1 , 0 8}$ & $\mathbf{1 , 1 7}$ & $\mathbf{1 , 3 4}$ & $\mathbf{1 , 4 1}$ \\
\hline $\mathbf{8 . 9}$ & $\mathbf{1 , 1 1}$ & $\mathbf{1 , 1 1}$ & $\mathbf{1 , 2 1}$ & $\mathbf{1 , 3 7}$ & $\mathbf{1 , 4 4}$ \\
\hline
\end{tabular}

Tabela 8.1: Atlas / Klein - Soma de vetores $(10 \leq n \leq 90)$

Para vetores entre 100 e 900 elementos a Klein passa a ganhar em quase todos os casos. 


\begin{tabular}{|c|c|c|c|c|c|}
\hline $\begin{array}{c}\text { Tempo Atlas } \\
\text { Tempo Klein }\end{array}$ & \multicolumn{5}{|c|}{ Número de elementos } \\
\hline Função & $\mathbf{1 0 0}$ & $\mathbf{3 0 0}$ & $\mathbf{5 0 0}$ & $\mathbf{7 0 0}$ & $\mathbf{9 0 0}$ \\
\hline $\mathbf{8 . 1}$ & $\mathbf{2 , 5 2}$ & 0,81 & 0,90 & 0,97 & 0,98 \\
\hline $\mathbf{8 . 2}$ & 0,74 & 0,94 & $\mathbf{1 , 0 4}$ & $\mathbf{1 , 0 4}$ & $\mathbf{1 , 0 7}$ \\
\hline $\mathbf{8 . 3}$ & $\mathbf{1 , 0 0}$ & $\mathbf{1 , 3 0}$ & $\mathbf{1 , 4 2}$ & $\mathbf{1 , 3 7}$ & $\mathbf{1 , 4 2}$ \\
\hline $\mathbf{8 . 4}$ & $\mathbf{1 , 1 2}$ & $\mathbf{1 , 3 1}$ & $\mathbf{1 , 4 1}$ & $\mathbf{1 , 4 1}$ & $\mathbf{1 , 4 2}$ \\
\hline $\mathbf{8 . 5}$ & 4,80 & $\mathbf{1 , 4 0}$ & $\mathbf{1 , 4 4}$ & $\mathbf{1 , 4 5}$ & $\mathbf{1 , 4 6}$ \\
\hline $\mathbf{8 . 6}$ & $\mathbf{1 , 2 9}$ & $\mathbf{1 , 3 9}$ & $\mathbf{1 , 3 9}$ & $\mathbf{1 , 4 4}$ & $\mathbf{1 , 4 5}$ \\
\hline $\mathbf{8 . 7}$ & $\mathbf{1 , 3 4}$ & $\mathbf{1 , 4 4}$ & $\mathbf{1 , 4 3}$ & $\mathbf{1 , 4 7}$ & $\mathbf{1 , 4 6}$ \\
\hline $\mathbf{8 . 8}$ & $\mathbf{1 , 3 5}$ & $\mathbf{1 , 4 2}$ & $\mathbf{1 , 4 1}$ & $\mathbf{1 , 4 5}$ & $\mathbf{1 , 4 7}$ \\
\hline $\mathbf{8 . 9}$ & $\mathbf{1 , 3 8}$ & $\mathbf{1 , 4 6}$ & $\mathbf{1 , 4 1}$ & $\mathbf{1 , 4 7}$ & $\mathbf{1 , 4 9}$ \\
\hline
\end{tabular}

Tabela 8.2: Atlas / Klein - Soma de vetores $(100 \leq n \leq 900)$

Já para vetores entre 1000 e 9000 elementos a ATLAS ganha somente quando somamos apenas dois elementos.

\begin{tabular}{|c|c|c|c|c|c|}
\hline $\begin{array}{c}\text { Tempo Atlas } \\
\text { Tempo Klein }\end{array}$ & \multicolumn{5}{|c|}{ Número de elementos } \\
\hline Função & $\mathbf{1 0 0 0}$ & $\mathbf{3 0 0 0}$ & $\mathbf{5 0 0 0}$ & $\mathbf{7 0 0 0}$ & $\mathbf{9 0 0 0}$ \\
\hline $\mathbf{8 . 1}$ & 0,94 & 0,95 & 0,94 & 0,93 & 0,89 \\
\hline $\mathbf{8 . 2}$ & $\mathbf{1 , 0 8}$ & $\mathbf{1 , 1 0}$ & $\mathbf{1 , 0 9}$ & $\mathbf{1 , 0 4}$ & $\mathbf{1 , 0 0}$ \\
\hline $\mathbf{8 . 3}$ & $\mathbf{1 , 3 8}$ & $\mathbf{1 , 3 6}$ & $\mathbf{1 , 3 7}$ & $\mathbf{1 , 2 3}$ & $\mathbf{1 , 1 3}$ \\
\hline $\mathbf{8 . 4}$ & $\mathbf{1 , 4 6}$ & $\mathbf{1 , 4 5}$ & $\mathbf{1 , 4 0}$ & $\mathbf{1 , 2 7}$ & $\mathbf{1 , 2 2}$ \\
\hline $\mathbf{8 . 5}$ & $\mathbf{1 , 4 6}$ & $\mathbf{1 , 4 5}$ & $\mathbf{1 , 3 8}$ & $\mathbf{1 , 3 7}$ & $\mathbf{1 , 2 5}$ \\
\hline $\mathbf{8 . 6}$ & $\mathbf{1 , 4 5}$ & $\mathbf{1 , 4 5}$ & $\mathbf{1 , 4 0}$ & $\mathbf{1 , 3 0}$ & $\mathbf{1 , 2 8}$ \\
\hline $\mathbf{8 . 7}$ & $\mathbf{1 , 4 5}$ & $\mathbf{1 , 4 5}$ & $\mathbf{1 , 3 8}$ & $\mathbf{1 , 3 3}$ & $\mathbf{1 , 3 0}$ \\
\hline $\mathbf{8 . 8}$ & $\mathbf{1 , 4 6}$ & $\mathbf{1 , 4 5}$ & $\mathbf{1 , 5 2}$ & $\mathbf{1 , 3 7}$ & $\mathbf{1 , 3 4}$ \\
\hline $\mathbf{8 . 9}$ & $\mathbf{1 , 4 6}$ & $\mathbf{1 , 4 5}$ & $\mathbf{1 , 4 2}$ & $\mathbf{1 , 3 7}$ & $\mathbf{1 , 3 4}$ \\
\hline
\end{tabular}

Tabela 8.3: Atlas / Klein - Soma de vetores (1000 $\leq n \leq 9000)$

\section{MKL / Klein}

Quando comparamos os tempos da Klein e da MKL, percebemos que quando são efetuadas várias somas em vetores grandes a Klein é superior. 


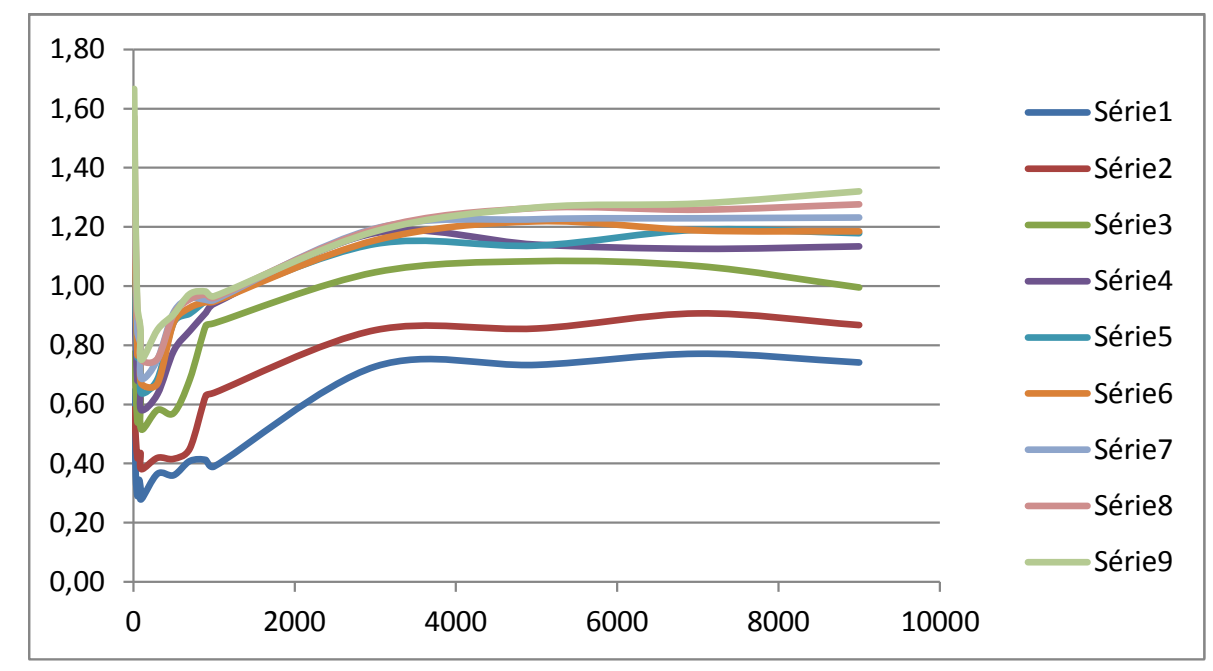

Figura 8.2: Soma vetores - MKL / Klein

Ao somar uma grande quantidade de vetores pequenos, entre 10 e 90 elementos, a Klein é competitiva apenas para os vetores menores.

\begin{tabular}{|c|c|c|c|c|c|}
\hline $\begin{array}{c}\text { Tempo MKL } \\
\text { Tempo Klein }\end{array}$ & \multicolumn{5}{|c|}{ Número de elementos } \\
\hline Função & $\mathbf{1 0}$ & $\mathbf{3 0}$ & $\mathbf{5 0}$ & $\mathbf{7 0}$ & $\mathbf{9 0}$ \\
\hline $\mathbf{8 . 1}$ & 0,64 & 0,36 & 0,29 & 0,34 & 0,32 \\
\hline $\mathbf{8 . 2}$ & 0,58 & 0,48 & 0,42 & 0,43 & 0,43 \\
\hline $\mathbf{8 . 3}$ & 0,77 & 0,65 & 0,54 & 0,57 & 0,62 \\
\hline $\mathbf{8 . 4}$ & 0,96 & 0,81 & 0,68 & 0,68 & 0,66 \\
\hline $\mathbf{8 . 5}$ & $\mathbf{1 , 1 3}$ & 0,89 & 0,76 & 0,78 & 0,72 \\
\hline $\mathbf{8 . 6}$ & $\mathbf{1 , 2 6}$ & 0,99 & 0,77 & 0,80 & 0,76 \\
\hline $\mathbf{8 . 7}$ & $\mathbf{1 , 3 9}$ & $\mathbf{1 , 1 0}$ & 0,85 & 0,82 & 0,80 \\
\hline $\mathbf{8 . 8}$ & $\mathbf{1 , 5 7}$ & $\mathbf{1 , 1 5}$ & 0,90 & 0,88 & 0,83 \\
\hline $\mathbf{8 . 9}$ & $\mathbf{1 , 6 7}$ & $\mathbf{1 , 1 9}$ & 0,93 & 0,89 & 0,85 \\
\hline
\end{tabular}

Tabela 8.4: $M K L /$ Klein - Soma de vetores $(10 \leq n \leq 90)$

Ao aumentar o tamanho dos vetores para entre 100 e 900 elementos, notamos que a medida que o número de elementos aumentamos a Klein volta a ser competitiva para soma de vários vetores ao mesmo tempo. 


\begin{tabular}{|c|c|c|c|c|c|}
\hline $\begin{array}{c}\text { Tempo MKL } \\
\text { Tempo Klein }\end{array}$ & \multicolumn{5}{|c|}{ Número de elementos } \\
\hline Função & $\mathbf{1 0 0}$ & $\mathbf{3 0 0}$ & $\mathbf{5 0 0}$ & $\mathbf{7 0 0}$ & $\mathbf{9 0 0}$ \\
\hline $\mathbf{8 . 1}$ & 0,28 & 0,37 & 0,36 & 0,41 & 0,41 \\
\hline $\mathbf{8 . 2}$ & 0,38 & 0,42 & 0,42 & 0,45 & 0,63 \\
\hline $\mathbf{8 . 3}$ & 0,51 & 0,58 & 0,57 & 0,68 & 0,87 \\
\hline $\mathbf{8 . 4}$ & 0,58 & 0,63 & 0,78 & 0,85 & 0,91 \\
\hline $\mathbf{8 . 5}$ & 0,64 & 0,69 & 0,88 & 0,91 & 0,95 \\
\hline $\mathbf{8 . 6}$ & 0,67 & 0,67 & 0,88 & 0,93 & 0,95 \\
\hline $\mathbf{8 . 7}$ & 0,69 & 0,75 & 0,91 & 0,96 & 0,95 \\
\hline $\mathbf{8 . 8}$ & 0,75 & 0,75 & 0,90 & 0,96 & 0,97 \\
\hline $\mathbf{8 . 9}$ & 0,75 & 0,85 & 0,90 & 0,97 & 0,98 \\
\hline
\end{tabular}

Tabela 8.5: $M K L /$ Klein - Soma de vetores $(100 \leq n \leq 900)$

Esse comportamento se evidencia ao analisarmos que a partir de 3000 elementos a Klein perde apenas ao somar apenas dois elementos, ganhando ou sendo competitiva nos outros casos.

\begin{tabular}{|c|c|c|c|c|c|}
\hline $\begin{array}{c}\text { Tempo MKL } \\
\text { Tempo Klein }\end{array}$ & \multicolumn{5}{|c|}{ Número de elementos } \\
\hline Função & $\mathbf{1 0 0 0}$ & $\mathbf{3 0 0 0}$ & $\mathbf{5 0 0 0}$ & $\mathbf{7 0 0 0}$ & $\mathbf{9 0 0 0}$ \\
\hline $\mathbf{8 . 1}$ & 0,39 & 0,73 & 0,73 & 0,77 & 0,74 \\
\hline $\mathbf{8 . 2}$ & 0,64 & 0,85 & 0,86 & 0,91 & 0,87 \\
\hline $\mathbf{8 . 3}$ & 0,87 & $\mathbf{1 , 0 5}$ & $\mathbf{1 , 0 8}$ & $\mathbf{1 , 0 7}$ & $\mathbf{1 , 0 0}$ \\
\hline $\mathbf{8 . 4}$ & 0,94 & $\mathbf{1 , 1 8}$ & $\mathbf{1 , 1 4}$ & $\mathbf{1 , 1 3}$ & $\mathbf{1 , 1 3}$ \\
\hline $\mathbf{8 . 5}$ & 0,95 & $\mathbf{1 , 1 4}$ & $\mathbf{1 , 1 4}$ & $\mathbf{1 , 1 9}$ & $\mathbf{1 , 1 8}$ \\
\hline $\mathbf{8 . 6}$ & 0,95 & $\mathbf{1 , 1 6}$ & $\mathbf{1 , 2 2}$ & $\mathbf{1 , 1 9}$ & $\mathbf{1 , 1 9}$ \\
\hline $\mathbf{8 . 7}$ & 0,95 & $\mathbf{1 , 1 9}$ & $\mathbf{1 , 2 3}$ & $\mathbf{1 , 2 3}$ & $\mathbf{1 , 2 3}$ \\
\hline $\mathbf{8 . 8}$ & 0,96 & $\mathbf{1 , 1 9}$ & $\mathbf{1 , 2 6}$ & $\mathbf{1 , 2 6}$ & $\mathbf{1 , 2 8}$ \\
\hline $\mathbf{8 . 9}$ & 0,97 & $\mathbf{1 , 1 9}$ & $\mathbf{1 , 2 7}$ & $\mathbf{1 , 2 8}$ & $\mathbf{1 , 3 2}$ \\
\hline
\end{tabular}

Tabela 8.6: $M K L /$ Klein - Soma de vetores $(1000 \leq n \leq 9000)$

\section{Expression Template / Klein}

Expression template é a estratégia utilizada pela Klein para efetuar soma de vetores. Porém a Klein possui toda uma estrutura que permite a utilização dessa estratégia, que não é otimizada $100 \%$ pelo compilador. Portanto esperamos que os tempos da estratégia expression template sejam um pouco melhores que os da Klein.

De fato, nesse caso a Klein perde praticamente em todos os casos. Mas 


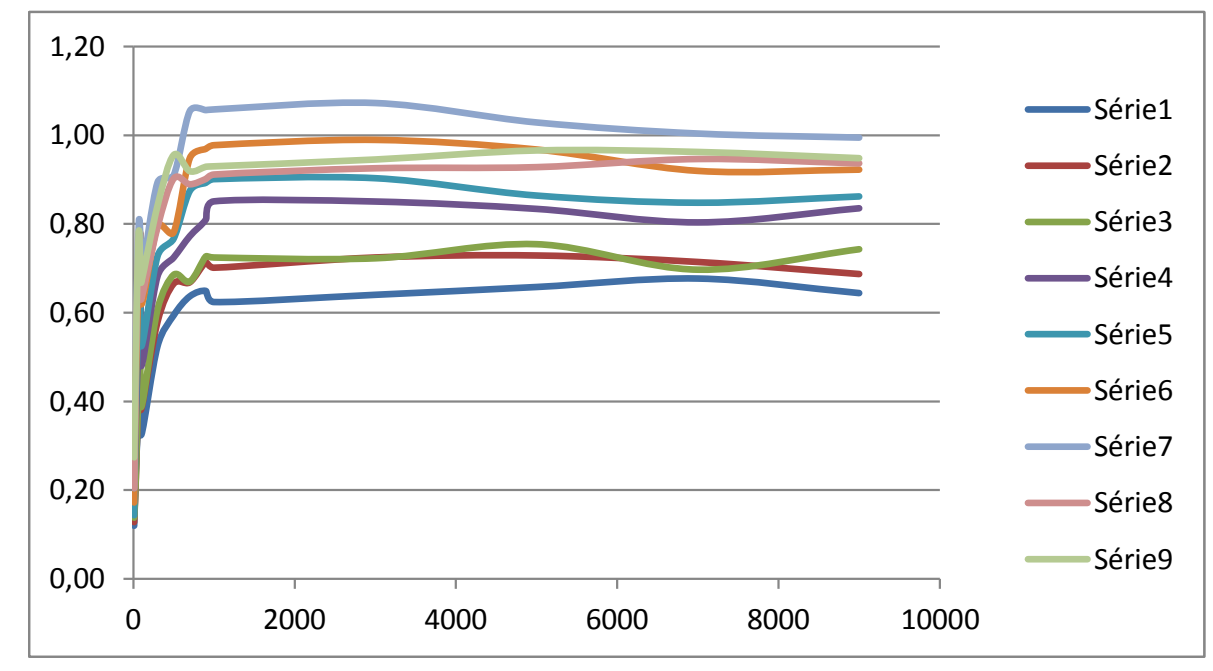

Figura 8.3: Soma vetores - Expression / Klein

Para vetores pequenos, entre 10 e 90 elementos, o tempo de chamada de função da Klein toma um tempo considerável, que vai diminuindo a medida que aumentamos o número de elementos e a quantidade de vetores sendo somados.

\begin{tabular}{|c|c|c|c|c|c|}
\hline $\begin{array}{c}\text { Tempo Expression } \\
\text { Tempo Klein }\end{array}$ & \multicolumn{5}{|c|}{ Número de elementos } \\
\hline Função & $\mathbf{1 0}$ & $\mathbf{3 0}$ & $\mathbf{5 0}$ & $\mathbf{7 0}$ & $\mathbf{9 0}$ \\
\hline $\mathbf{8 . 1}$ & 0,12 & 0,24 & 0,32 & 0,40 & 0,42 \\
\hline $\mathbf{8 . 2}$ & 0,13 & 0,22 & 0,35 & 0,43 & 0,45 \\
\hline $\mathbf{8 . 3}$ & 0,14 & 0,23 & 0,35 & 0,43 & 0,52 \\
\hline $\mathbf{8 . 4}$ & 0,17 & 0,35 & 0,47 & 0,57 & 0,63 \\
\hline $\mathbf{8 . 5}$ & 0,14 & 0,39 & 0,51 & 0,61 & 0,68 \\
\hline $\mathbf{8 . 6}$ & 0,17 & 0,43 & 0,58 & 0,73 & 0,78 \\
\hline $\mathbf{8 . 7}$ & 0,20 & 0,51 & 0,69 & 0,81 & 0,76 \\
\hline $\mathbf{8 . 8}$ & 0,21 & 0,48 & 0,65 & 0,75 & 0,65 \\
\hline $\mathbf{8 . 9}$ & 0,27 & 0,50 & 0,69 & 0,79 & 0,68 \\
\hline
\end{tabular}

Tabela 8.7: Expression Template / Klein - Soma de vetores $(10 \leq n \leq 90)$

Esse comportamento se repete para vetores entre 100 e 900 elementos. 


\begin{tabular}{|c|c|c|c|c|c|}
\hline $\begin{array}{c}\text { Tempo Expression } \\
\text { Tempo Klein }\end{array}$ & \multicolumn{5}{|c|}{ Número de elementos } \\
\hline Função & $\mathbf{1 0 0}$ & $\mathbf{3 0 0}$ & $\mathbf{5 0 0}$ & $\mathbf{7 0 0}$ & $\mathbf{9 0 0}$ \\
\hline $\mathbf{8 . 1}$ & 0,33 & 0,52 & 0,59 & 0,64 & 0,65 \\
\hline $\mathbf{8 . 2}$ & 0,38 & 0,58 & 0,67 & 0,67 & 0,71 \\
\hline $\mathbf{8 . 3}$ & 0,39 & 0,61 & 0,69 & 0,67 & 0,73 \\
\hline $\mathbf{8 . 4}$ & 0,48 & 0,68 & 0,72 & 0,77 & 0,81 \\
\hline $\mathbf{8 . 5}$ & 0,52 & 0,73 & 0,77 & 0,87 & 0,89 \\
\hline $\mathbf{8 . 6}$ & 0,62 & 0,79 & 0,78 & 0,95 & 0,97 \\
\hline $\mathbf{8 . 7}$ & 0,69 & 0,89 & 0,91 & $\mathbf{1 , 0 5}$ & $\mathbf{1 , 0 6}$ \\
\hline $\mathbf{8 . 8}$ & 0,63 & 0,79 & 0,90 & 0,89 & 0,90 \\
\hline $\mathbf{8 . 9}$ & 0,67 & 0,84 & 0,96 & 0,92 & 0,93 \\
\hline
\end{tabular}

Tabela 8.8: Expression Template / Klein - Soma de vetores $(100 \leq n \leq 900)$

Assim como para vetores entre 1000 e 9000 elementos.

\begin{tabular}{|c|c|c|c|c|c|}
\hline$\frac{5}{\text { Tempo Expression }}$ Tempo Klein & \multicolumn{5}{|c|}{ Número de elementos } \\
\hline Função & $\mathbf{1 0 0 0}$ & $\mathbf{3 0 0 0}$ & $\mathbf{5 0 0 0}$ & $\mathbf{7 0 0 0}$ & $\mathbf{9 0 0 0}$ \\
\hline $\mathbf{8 . 1}$ & 0,62 & 0,64 & 0,66 & 0,68 & 0,64 \\
\hline $\mathbf{8 . 2}$ & 0,70 & 0,72 & 0,73 & 0,71 & 0,69 \\
\hline $\mathbf{8 . 3}$ & 0,72 & 0,72 & 0,75 & 0,70 & 0,74 \\
\hline $\mathbf{8 . 4}$ & 0,85 & 0,85 & 0,83 & 0,80 & 0,84 \\
\hline $\mathbf{8 . 5}$ & 0,90 & 0,90 & 0,86 & 0,85 & 0,86 \\
\hline $\mathbf{8 . 6}$ & 0,98 & 0,99 & 0,97 & 0,92 & 0,92 \\
\hline $\mathbf{8 . 7}$ & $\mathbf{1 , 0 6}$ & $\mathbf{1 , 0 7}$ & $\mathbf{1 , 0 3}$ & $\mathbf{1 , 0 0}$ & 0,99 \\
\hline $\mathbf{8 . 8}$ & 0,91 & 0,93 & 0,93 & 0,95 & 0,94 \\
\hline $\mathbf{8 . 9}$ & 0,93 & 0,95 & 0,97 & 0,96 & 0,95 \\
\hline
\end{tabular}

Tabela 8.9: Expression Template / Klein - Soma de vetores $(1000 \leq n \leq 9000)$

\section{Dummy / Klein}

O código dummy é o algoritmo ingênuo, que efetua a soma de cada um dos vetores separadamente.

Exceto pela soma de dois vetores, existe um número de elementos em que a Klein ganhará dessa estratégia em todos os casos de soma de vetores testados. 


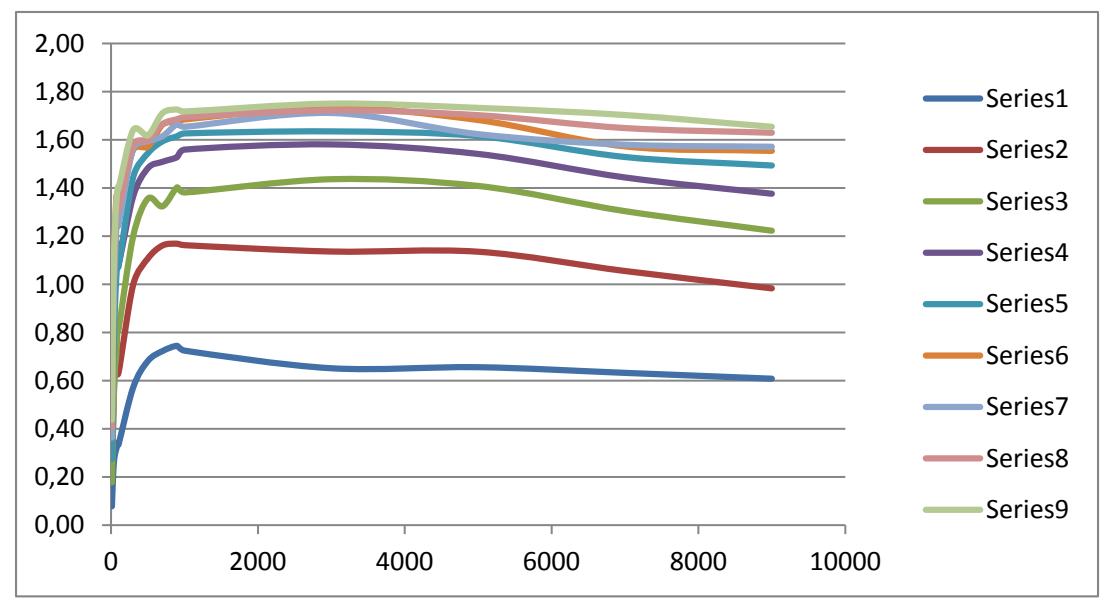

Figura 8.4: Soma vetores - Dummy / Klein

Para os casos de vetores pequenos ele possui um desempenho melhor que o da Klein. Porém a medida que aumentamos os vetores a vantagem diminui e essa estratégia passa a ser mais lenta em mais casos.

\begin{tabular}{|c|c|c|c|c|c|}
\hline $\begin{array}{c}\text { Tempo Dummy } \\
\text { Tempo Klein }\end{array}$ & \multicolumn{5}{|c|}{ Número de elementos } \\
\hline Função & $\mathbf{1 0}$ & $\mathbf{3 0}$ & $\mathbf{5 0}$ & $\mathbf{7 0}$ & $\mathbf{9 0}$ \\
\hline $\mathbf{8 . 1}$ & 0,08 & 0,23 & 0,29 & 0,32 & 0,34 \\
\hline $\mathbf{8 . 2}$ & 0,27 & 0,47 & 0,70 & 0,66 & 0,73 \\
\hline $\mathbf{8 . 3}$ & 0,18 & 0,46 & 0,64 & 0,71 & 0,83 \\
\hline $\mathbf{8 . 4}$ & 0,32 & 0,88 & 0,98 & $\mathbf{1 , 1 1}$ & $\mathbf{1 , 1 7}$ \\
\hline $\mathbf{8 . 5}$ & 0,28 & 0,69 & 0,90 & $\mathbf{1 , 0 3}$ & $\mathbf{1 , 1 2}$ \\
\hline $\mathbf{8 . 6}$ & 0,45 & $\mathbf{1 , 1 6}$ & $\mathbf{1 , 2 1}$ & $\mathbf{1 , 3 6}$ & $\mathbf{1 , 3 6}$ \\
\hline $\mathbf{8 . 7}$ & 0,36 & 0,89 & $\mathbf{1 , 0 8}$ & $\mathbf{1 , 2 4}$ & $\mathbf{1 , 2 9}$ \\
\hline $\mathbf{8 . 8}$ & 0,41 & 0,95 & $\mathbf{1 , 1 6}$ & $\mathbf{1 , 3 1}$ & $\mathbf{1 , 3 6}$ \\
\hline $\mathbf{8 . 9}$ & 0,44 & 0,99 & $\mathbf{1 , 2 2}$ & $\mathbf{1 , 3 4}$ & $\mathbf{1 , 4 0}$ \\
\hline
\end{tabular}

Tabela 8.10: Dummy / Klein - Soma de vetores $(10 \leq n \leq 90)$

A partir de 300 elementos a Klein só não é mais rápida nos casos em que somamos apenas dois vetores. Mas isso já era esperado, pois para a soma de apenas dois vetores a estratégia da Klein é igual à dummy. A vantagem dessa última são devido o tempo de chamada de funções da Klein. 


\begin{tabular}{|c|c|c|c|c|c|}
\hline $\begin{array}{c}\text { Tempo Dummy } \\
\text { Tempo Klein }\end{array}$ & \multicolumn{5}{|c|}{ Número de elementos } \\
\hline Função & $\mathbf{1 0 0}$ & $\mathbf{3 0 0}$ & $\mathbf{5 0 0}$ & $\mathbf{7 0 0}$ & $\mathbf{9 0 0}$ \\
\hline $\mathbf{8 . 1}$ & 0,33 & 0,57 & 0,68 & 0,72 & 0,75 \\
\hline $\mathbf{8 . 2}$ & 0,63 & $\mathbf{1 , 0 0}$ & $\mathbf{1 , 1 1}$ & $\mathbf{1 , 1 6}$ & $\mathbf{1 , 1 7}$ \\
\hline $\mathbf{8 . 3}$ & 0,81 & $\mathbf{1 , 2 0}$ & $\mathbf{1 , 3 6}$ & $\mathbf{1 , 3 2}$ & $\mathbf{1 , 4 0}$ \\
\hline $\mathbf{8 . 4}$ & $\mathbf{1 , 0 8}$ & $\mathbf{1 , 3 7}$ & $\mathbf{1 , 4 8}$ & $\mathbf{1 , 5 1}$ & $\mathbf{1 , 5 3}$ \\
\hline $\mathbf{8 . 5}$ & $\mathbf{1 , 0 7}$ & $\mathbf{1 , 4 5}$ & $\mathbf{1 , 5 4}$ & $\mathbf{1 , 5 9}$ & $\mathbf{1 , 6 1}$ \\
\hline $\mathbf{8 . 6}$ & $\mathbf{1 , 3 3}$ & $\mathbf{1 , 5 6}$ & $\mathbf{1 , 5 7}$ & $\mathbf{1 , 6 6}$ & $\mathbf{1 , 6 7}$ \\
\hline $\mathbf{8 . 7}$ & $\mathbf{1 , 2 5}$ & $\mathbf{1 , 5 6}$ & $\mathbf{1 , 5 9}$ & $\mathbf{1 , 6 2}$ & $\mathbf{1 , 6 7}$ \\
\hline $\mathbf{8 . 8}$ & $\mathbf{1 , 3 1}$ & $\mathbf{1 , 5 8}$ & $\mathbf{1 , 6 0}$ & $\mathbf{1 , 6 7}$ & $\mathbf{1 , 6 9}$ \\
\hline $\mathbf{8 . 9}$ & $\mathbf{1 , 4 0}$ & $\mathbf{1 , 6 4}$ & $\mathbf{1 , 6 2}$ & $\mathbf{1 , 7 1}$ & $\mathbf{1 , 7 3}$ \\
\hline
\end{tabular}

Tabela 8.11: Dummy / Klein - Soma de vetores $(100 \leq n \leq 900)$

Para vetores entre 1000 e 9000 elementos a Klein continua ganhando. Mas a partir de 5000 elementos notamos uma redução na vantagem da Klein.

\begin{tabular}{|c|c|c|c|c|c|}
\hline $\begin{array}{c}\text { Tempo Dummy } \\
\text { Tempo Klein }\end{array}$ & \multicolumn{5}{|c|}{ Número de elementos } \\
\hline Função & $\mathbf{1 0 0 0}$ & $\mathbf{3 0 0 0}$ & $\mathbf{5 0 0 0}$ & $\mathbf{7 0 0 0}$ & $\mathbf{9 0 0 0}$ \\
\hline $\mathbf{8 . 1}$ & 0,73 & 0,65 & 0,50 & 0,48 & 0,47 \\
\hline $\mathbf{8 . 2}$ & $\mathbf{1 , 1 6}$ & $\mathbf{1 , 1 4}$ & 0,86 & 0,79 & 0,73 \\
\hline $\mathbf{8 . 3}$ & $\mathbf{1 , 3 8}$ & $\mathbf{1 , 4 4}$ & $\mathbf{1 , 0 6}$ & 0,97 & 0,91 \\
\hline $\mathbf{8 . 4}$ & $\mathbf{1 , 5 6}$ & $\mathbf{1 , 5 8}$ & $\mathbf{1 , 1 3}$ & $\mathbf{1 , 0 6}$ & $\mathbf{1 , 0 0}$ \\
\hline $\mathbf{8 . 5}$ & $\mathbf{1 , 6 3}$ & $\mathbf{1 , 6 4}$ & $\mathbf{1 , 1 8}$ & $\mathbf{1 , 1 0}$ & $\mathbf{1 , 0 7}$ \\
\hline $\mathbf{8 . 6}$ & $\mathbf{1 , 6 8}$ & $\mathbf{1 , 7 3}$ & $\mathbf{1 , 2 0}$ & $\mathbf{1 , 1 3}$ & $\mathbf{1 , 1 2}$ \\
\hline $\mathbf{8 . 7}$ & $\mathbf{1 , 6 5}$ & $\mathbf{1 , 7 1}$ & $\mathbf{1 , 2 1}$ & $\mathbf{1 , 1 9}$ & $\mathbf{1 , 1 7}$ \\
\hline $\mathbf{8 . 8}$ & $\mathbf{1 , 6 9}$ & $\mathbf{1 , 7 2}$ & $\mathbf{1 , 2 9}$ & $\mathbf{1 , 2 4}$ & $\mathbf{1 , 2 2}$ \\
\hline $\mathbf{8 . 9}$ & $\mathbf{1 , 7 2}$ & $\mathbf{1 , 7 5}$ & $\mathbf{1 , 3 1}$ & $\mathbf{1 , 2 6}$ & $\mathbf{1 , 2 4}$ \\
\hline
\end{tabular}

Tabela 8.12: Dummy / Klein - Soma de vetores $(1000 \leq n \leq 9000)$

\subsection{Soma de vetor vezes escalar}

Criamos testes para o cálculo das expressões abaixo:

$$
\begin{gathered}
r e s p=k_{1} * a+k_{2} * b \\
r e s p=k_{1} * a+k_{2} * b+k_{3} * c \\
r e s p=k_{1} * a+k_{2} * b+k_{3} * c+k_{4} * d, \\
r e s p=k_{1} * a+k_{2} * b+k_{3} * c+k_{4} * d+k_{5} * e \\
r e s p=k_{1} * a+k_{2} * b+k_{3} * c+k_{4} * d+k_{5} * e+k_{6} * f \\
r e s p=k_{1} * a+k_{2} * b+k_{3} * c+k_{4} * d+k_{5} * e+k_{6} * f+k_{7} * g, \\
r e s p=k_{1} * a+k_{2} * b+k_{3} * c+k_{4} * d+k_{5} * e+k_{6} * f+k_{7} * g+k_{8} * h, \\
r e s p=k_{1} * a+k_{2} * b+k_{3} * c+k_{4} * d+k_{5} * e+k_{6} * f+k_{7} * g+k_{8} * h+k_{9} * i, \\
r e s p=k_{1} * a+k_{2} * b+k_{3} * c+k_{4} * d+k_{5} * e+k_{6} * f+k_{7} * g+k_{8} * h+k_{9} * i+k_{10} * j,
\end{gathered}
$$

onde resp, $a, b, c, d, e, f, g, h, i$ e $j$ são vetores, e $k_{1}, k_{2}, k_{3}, k_{4}, k_{5}, k_{6}, k_{7}, k_{8}, k_{9}$ e $k_{10}$ são escalares.

Os códigos das bibliotecas MKL, Atlas e Klein para a expressão resp $=a+b+c+d+e+f+$ $g+h+i+j$ são mostrados a seguir. O código para as bibliotecas MKL e ATLAS é o mesmo e fica 
desta forma:

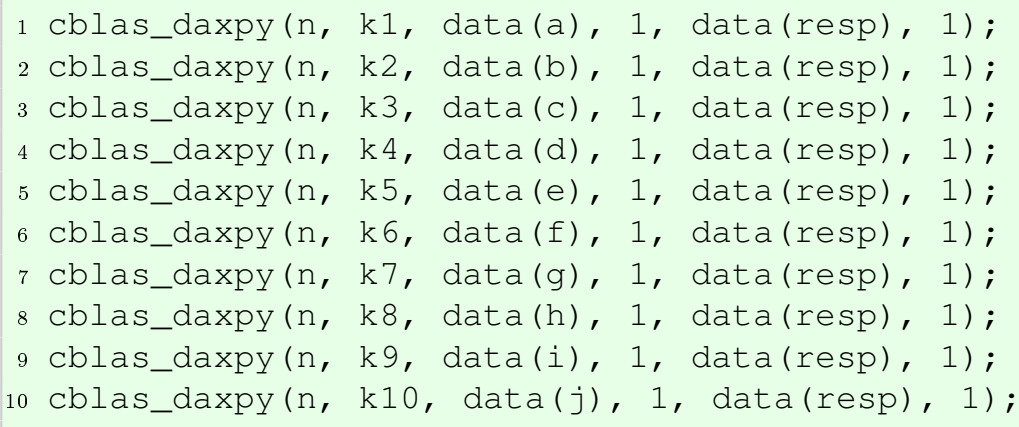

A estratégia expression template fica dessa maneira:

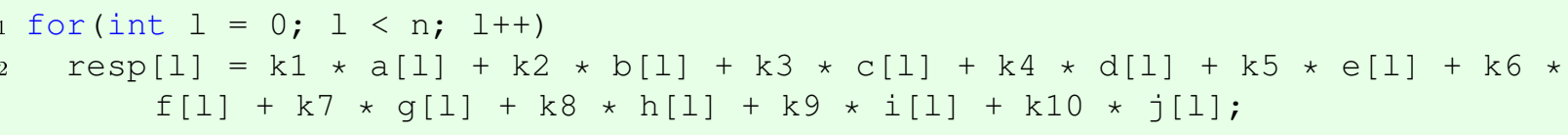

O código dummy fica dessa maneira:

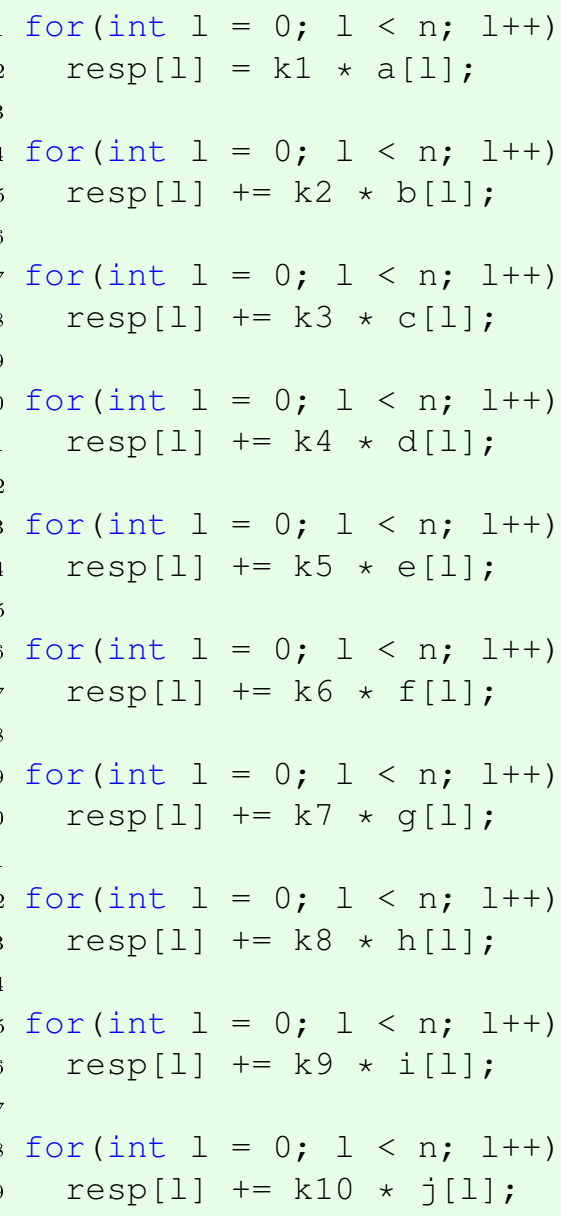

E o código da Klein, fica desta outra forma:

1 resp $=k 1 \star a+k 2 \star b+k 3 \star c+k 4 \star d+k 5 * e+k 6 \star f+k 7 \star g+k 8 \star h$ $+k 9 \star i+k 10 \star j ;$

\section{Atlas / Klein}

Quando efetuamos a soma de vetor pre multiplicando por escalar, o resultado é parecido com a soma de vetores. A medida que o número de elementos e a quantidade de vetores sendo somados aumentam a Klein vai melhorando na comparação. 


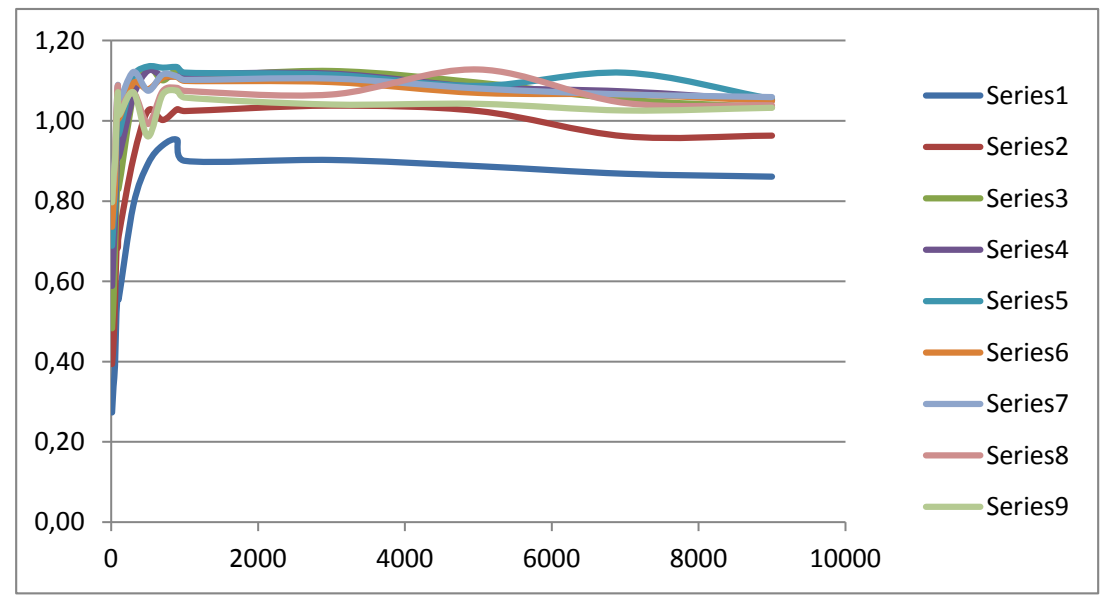

Figura 8.5: Multiplica escalar e soma vetores - Atlas / Klein

\begin{tabular}{|c|c|c|c|c|c|}
\hline$\frac{\text { Tempo Atlas }}{\text { Tempo Klein }}$ & \multicolumn{5}{|c|}{ Número de elementos } \\
\hline Função & 10 & 30 & 50 & 70 & 90 \\
\hline 8.10 & 0,26 & 0,32 & 0,35 & 0,45 & 0,48 \\
\hline 8.11 & 0,39 & 0,44 & 0,51 & 0,63 & 0,69 \\
\hline 8.12 & 0,48 & 0,55 & 0,63 & 0,73 & 0,84 \\
\hline 8.13 & 0,60 & 0,67 & 0,75 & 0,88 & 0,98 \\
\hline 8.14 & 0,70 & 0,75 & 0,82 & 0,95 & 1,03 \\
\hline 8.15 & 0,74 & 0,80 & 0,84 & 1,00 & 1,04 \\
\hline 8.16 & 0,82 & 0,88 & 0,91 & 1,03 & 1,09 \\
\hline 8.17 & 0,82 & 0,84 & 0,91 & 1,02 & $\mathbf{1 , 0 9}$ \\
\hline 8.18 & 0,79 & 0,86 & 0,90 & 1,01 & 1,07 \\
\hline
\end{tabular}

Tabela 8.13: Atlas / Klein - Soma de vetor vezes escalar $(10 \leq n \leq 90)$

Quando aumentamos o número de elementos dos vetores, entre 100 e 900 elementos, a Klein supera a Atlas na maioria dos casos.

\begin{tabular}{|c|c|c|c|c|c|}
\hline $\begin{array}{c}\text { Tempo Atlas } \\
\text { Tempo Klein }\end{array}$ & \multicolumn{5}{|c|}{ Número de elementos } \\
\hline Função & $\mathbf{1 0 0}$ & $\mathbf{3 0 0}$ & $\mathbf{5 0 0}$ & $\mathbf{7 0 0}$ & $\mathbf{9 0 0}$ \\
\hline $\mathbf{8 . 1 0}$ & 0,56 & 0,79 & 0,89 & 0,94 & 0,95 \\
\hline $\mathbf{8 . 1 1}$ & 0,71 & 0,91 & $\mathbf{1 , 0 3}$ & $\mathbf{1 , 0 0}$ & $\mathbf{1 , 0 3}$ \\
\hline $\mathbf{8 . 1 2}$ & 0,83 & $\mathbf{1 , 0 6}$ & $\mathbf{1 , 1 3}$ & $\mathbf{1 , 1 0}$ & $\mathbf{1 , 1 3}$ \\
\hline $\mathbf{8 . 1 3}$ & 0,91 & $\mathbf{1 , 0 6}$ & $\mathbf{1 , 1 2}$ & $\mathbf{1 , 1 1}$ & $\mathbf{1 , 1 1}$ \\
\hline $\mathbf{8 . 1 4}$ & 0,97 & $\mathbf{1 , 1 1}$ & $\mathbf{1 , 1 4}$ & $\mathbf{1 , 1 3}$ & $\mathbf{1 , 1 3}$ \\
\hline $\mathbf{8 . 1 5}$ & $\mathbf{1 , 0 0}$ & $\mathbf{1 , 0 9}$ & $\mathbf{1 , 0 8}$ & $\mathbf{1 , 1 1}$ & $\mathbf{1 , 1 1}$ \\
\hline $\mathbf{8 . 1 6}$ & $\mathbf{1 , 0 5}$ & $\mathbf{1 , 1 2}$ & $\mathbf{1 , 0 7}$ & $\mathbf{1 , 1 2}$ & $\mathbf{1 , 1 1}$ \\
\hline $\mathbf{8 . 1 7}$ & $\mathbf{1 , 0 3}$ & $\mathbf{1 , 0 7}$ & 0,99 & $\mathbf{1 , 0 7}$ & $\mathbf{1 , 0 8}$ \\
\hline $\mathbf{8 . 1 8}$ & $\mathbf{1 , 0 1}$ & $\mathbf{1 , 0 7}$ & 0,96 & $\mathbf{1 , 0 7}$ & $\mathbf{1 , 0 8}$ \\
\hline
\end{tabular}

Tabela 8.14: Atlas / Klein - Soma de vetor vezes escalar $(100 \leq n \leq 900)$

Para vetores grandes entre 1000 e 3000 elementos a Klein é competitiva para soma de dois e 
três vetores.

\begin{tabular}{|c|c|c|c|c|c|}
\hline $\begin{array}{c}\text { Tempo Atlas } \\
\text { Tempo Klein }\end{array}$ & \multicolumn{5}{|c|}{ Número de elementos } \\
\hline Função & $\mathbf{1 0 0 0}$ & $\mathbf{3 0 0 0}$ & $\mathbf{5 0 0 0}$ & $\mathbf{7 0 0 0}$ & $\mathbf{9 0 0 0}$ \\
\hline $\mathbf{8 . 1 0}$ & 0,72 & 0,73 & 0,89 & 0,87 & 0,86 \\
\hline $\mathbf{8 . 1 1}$ & 0,96 & 0,98 & $\mathbf{1 , 0 2}$ & 0,96 & 0,96 \\
\hline $\mathbf{8 . 1 2}$ & $\mathbf{1 , 0 6}$ & $\mathbf{1 , 0 6}$ & $\mathbf{1 , 0 9}$ & $\mathbf{1 , 0 5}$ & $\mathbf{1 , 0 3}$ \\
\hline $\mathbf{8 . 1 3}$ & $\mathbf{1 , 1 2}$ & $\mathbf{1 , 1 2}$ & $\mathbf{1 , 0 8}$ & $\mathbf{1 , 0 7}$ & $\mathbf{1 , 0 5}$ \\
\hline $\mathbf{8 . 1 4}$ & $\mathbf{1 , 1 2}$ & $\mathbf{1 , 1 2}$ & $\mathbf{1 , 0 9}$ & $\mathbf{1 , 1 2}$ & $\mathbf{1 , 0 5}$ \\
\hline $\mathbf{8 . 1 5}$ & $\mathbf{1 , 1 0}$ & $\mathbf{1 , 1 0}$ & $\mathbf{1 , 0 7}$ & $\mathbf{1 , 0 7}$ & $\mathbf{1 , 0 5}$ \\
\hline $\mathbf{8 . 1 6}$ & $\mathbf{1 , 1 0}$ & $\mathbf{1 , 1 0}$ & $\mathbf{1 , 0 8}$ & $\mathbf{1 , 0 7}$ & $\mathbf{1 , 0 6}$ \\
\hline $\mathbf{8 . 1 7}$ & $\mathbf{1 , 0 8}$ & $\mathbf{1 , 0 7}$ & $\mathbf{1 , 1 3}$ & $\mathbf{1 , 0 4}$ & $\mathbf{1 , 0 4}$ \\
\hline $\mathbf{8 . 1 8}$ & $\mathbf{1 , 0 6}$ & $\mathbf{1 , 0 5}$ & $\mathbf{1 , 0 4}$ & $\mathbf{1 , 0 3}$ & $\mathbf{1 , 0 3}$ \\
\hline
\end{tabular}

Tabela 8.15: Atlas / Klein - Soma de vetor vezes escalar $(1000 \leq n \leq 9000)$

\section{MKL / Klein}

Neste caso, a Klein perde em quase todos os casos, exceto para vetores pequenos e várias operações sendo efetuadas.

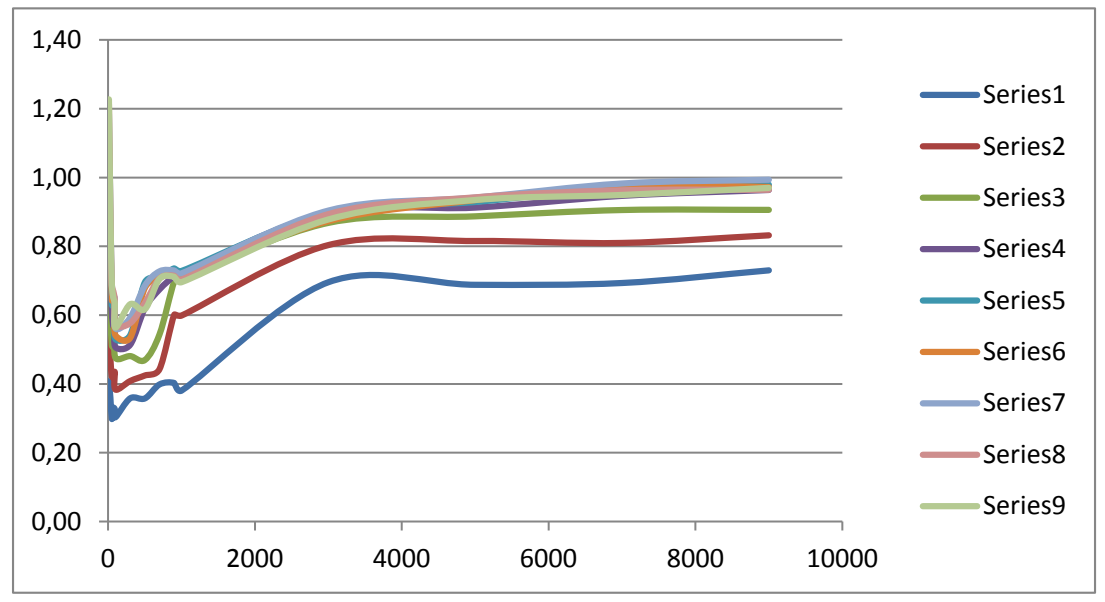

Figura 8.6: Multiplica escalar e soma vetores - MKL / Klein

Para os vetores entre 10 e 90 elementos a medida que aumentamos o número de elementos a MKL fica melhor que a Klein. 


\begin{tabular}{|c|c|c|c|c|c|}
\hline $\begin{array}{c}\text { Tempo MKL } \\
\text { Tempo Klein }\end{array}$ & \multicolumn{5}{|c|}{ Número de elementos } \\
\hline Função & $\mathbf{1 0}$ & $\mathbf{3 0}$ & $\mathbf{5 0}$ & $\mathbf{7 0}$ & $\mathbf{9 0}$ \\
\hline $\mathbf{8 . 1 0}$ & 0,39 & 0,34 & 0,27 & 0,29 & 0,28 \\
\hline $\mathbf{8 . 1 1}$ & 0,57 & 0,47 & 0,39 & 0,39 & 0,44 \\
\hline $\mathbf{8 . 1 2}$ & 0,76 & 0,59 & 0,47 & 0,46 & 0,52 \\
\hline $\mathbf{8 . 1 3}$ & 0,90 & 0,71 & 0,61 & 0,57 & 0,56 \\
\hline $\mathbf{8 . 1 4}$ & $\mathbf{1 , 0 2}$ & 0,79 & 0,65 & 0,63 & 0,60 \\
\hline $\mathbf{8 . 1 5}$ & $\mathbf{1 , 1 1}$ & 0,85 & 0,65 & 0,66 & 0,61 \\
\hline $\mathbf{8 . 1 6}$ & $\mathbf{1 , 2 1}$ & 0,91 & 0,69 & 0,66 & 0,65 \\
\hline $\mathbf{8 . 1 7}$ & $\mathbf{1 , 2 2}$ & 0,89 & 0,70 & 0,67 & 0,64 \\
\hline $\mathbf{8 . 1 8}$ & $\mathbf{1 , 2 2}$ & 0,89 & 0,69 & 0,65 & 0,63 \\
\hline
\end{tabular}

Tabela 8.16: $M K L /$ Klein - Soma de vetor vezes escalar $(10 \leq n \leq 90)$

Porém a partir de 100 elementos ao aumentarmos o número de elementos dos vetores a Klein começa a melhorar em relação à MKL.

\begin{tabular}{|c|c|c|c|c|c|}
\hline $\begin{array}{l}\text { Tempo MKL } \\
\text { Tempo Klein }\end{array}$ & \multicolumn{5}{|c|}{ Número de elementos } \\
\hline Função & 100 & 300 & 500 & 700 & 900 \\
\hline 8.10 & 0,30 & 0,36 & 0,36 & 0,40 & 0,40 \\
\hline 8.11 & 0,38 & 0,41 & 0,42 & 0,44 & 0,60 \\
\hline 8.12 & 0,48 & 0,48 & 0,47 & 0,54 & 0,70 \\
\hline 8.13 & 0,51 & 0,51 & 0,62 & 0,68 & 0,71 \\
\hline 8.14 & 0,53 & 0,54 & 0,70 & 0,71 & 0,74 \\
\hline 8.15 & 0,54 & 0,53 & 0,68 & 0,71 & 0,72 \\
\hline 8.16 & 0,56 & 0,59 & 0,69 & 0,73 & 0,73 \\
\hline 8.17 & 0,57 & 0,58 & 0,64 & 0,71 & 0,71 \\
\hline 8.18 & 0,56 & 0,63 & 0,62 & 0,71 & 0,71 \\
\hline
\end{tabular}

Tabela 8.17: $M K L /$ Klein - Soma de vetor vezes escalar $(100 \leq n \leq 900)$

Esse comportamento continua nos vetores entre 1000 e 9000 elementos.

\begin{tabular}{|c|c|c|c|c|c|}
\hline Tempo MKL & \multicolumn{5}{|c|}{ Número de elementos } \\
\hline Tempo Klein & \multicolumn{7}{|c|}{} \\
\hline Função & $\mathbf{1 0 0 0}$ & $\mathbf{3 0 0 0}$ & $\mathbf{5 0 0 0}$ & $\mathbf{7 0 0 0}$ & $\mathbf{9 0 0 0}$ \\
\hline $\mathbf{8 . 1 0}$ & 0,31 & 0,56 & 0,69 & 0,69 & 0,73 \\
\hline $\mathbf{8 . 1 1}$ & 0,56 & 0,76 & 0,82 & 0,81 & 0,83 \\
\hline $\mathbf{8 . 1 2}$ & 0,67 & 0,82 & 0,89 & 0,91 & 0,91 \\
\hline $\mathbf{8 . 1 3}$ & 0,72 & 0,90 & 0,91 & 0,95 & 0,96 \\
\hline $\mathbf{8 . 1 4}$ & 0,73 & 0,90 & 0,93 & 0,97 & 0,98 \\
\hline $\mathbf{8 . 1 5}$ & 0,72 & 0,88 & 0,93 & 0,98 & 0,97 \\
\hline $\mathbf{8 . 1 6}$ & 0,72 & 0,90 & 0,94 & 0,98 & 0,99 \\
\hline $\mathbf{8 . 1 7}$ & 0,70 & 0,90 & 0,94 & 0,96 & 0,96 \\
\hline $\mathbf{8 . 1 8}$ & 0,70 & 0,89 & 0,93 & 0,95 & 0,97 \\
\hline
\end{tabular}

Tabela 8.18: $M K L /$ Klein - Soma de vetor vezes escalar $(1000 \leq n \leq 9000)$

\section{Expression template $\mathrm{x}$ Klein}

Para vetores com grande quantidade de elementos a estratégia expression template é inferior a Klein quando efetuamos acima de 3 operações. 


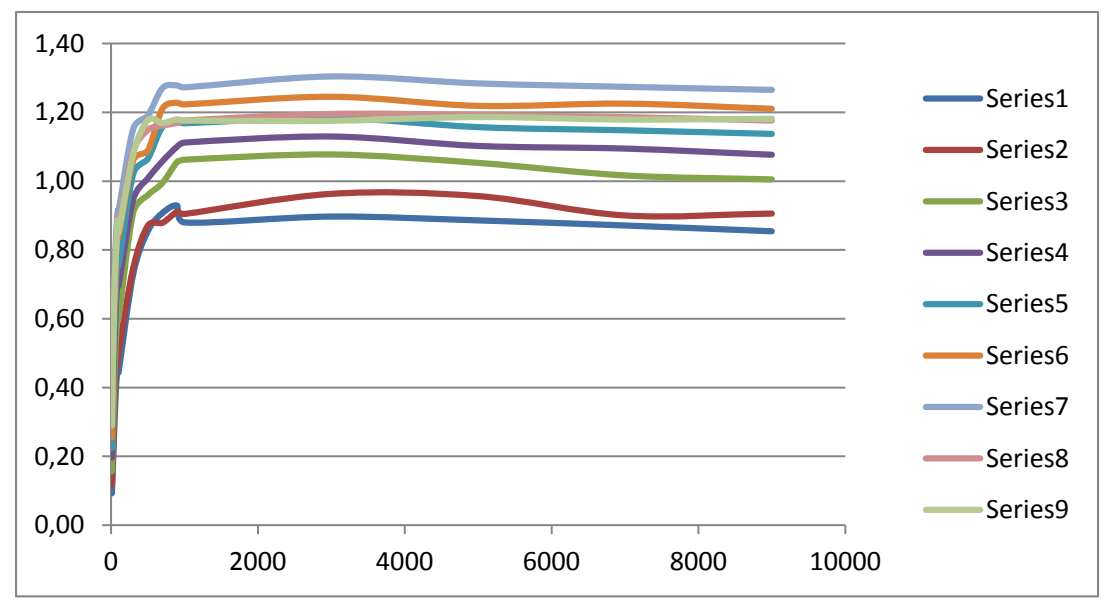

Figura 8.7: Multiplica escalar e soma vetores - Expression / Klein

A técnica expression template é melhor que a Klein para vetores entre 10 e 90 elementos, porém a medida que aumentamos o número de elementos a Klein começa a ser mais competitiva.

\begin{tabular}{|c|c|c|c|c|c|}
\hline $\begin{array}{c}\text { Tempo Expression } \\
\text { Tempo Klein }\end{array}$ & \multicolumn{5}{|c|}{ Número de elementos } \\
\hline Função & $\mathbf{1 0}$ & $\mathbf{3 0}$ & $\mathbf{5 0}$ & $\mathbf{7 0}$ & $\mathbf{9 0}$ \\
\hline $\mathbf{8 . 1 0}$ & 0,09 & 0,20 & 0,29 & 0,36 & 0,39 \\
\hline $\mathbf{8 . 1 1}$ & 0,12 & 0,24 & 0,35 & 0,43 & 0,48 \\
\hline $\mathbf{8 . 1 2}$ & 0,16 & 0,30 & 0,43 & 0,52 & 0,59 \\
\hline $\mathbf{8 . 1 3}$ & 0,20 & 0,43 & 0,55 & 0,67 & 0,72 \\
\hline $\mathbf{8 . 1 4}$ & 0,23 & 0,48 & 0,61 & 0,72 & 0,79 \\
\hline $\mathbf{8 . 1 5}$ & 0,26 & 0,54 & 0,66 & 0,81 & 0,87 \\
\hline $\mathbf{8 . 1 6}$ & 0,30 & 0,57 & 0,74 & 0,86 & 0,92 \\
\hline $\mathbf{8 . 1 7}$ & 0,30 & 0,57 & 0,73 & 0,83 & 0,89 \\
\hline $\mathbf{8 . 1 8}$ & 0,29 & 0,56 & 0,72 & 0,81 & 0,88 \\
\hline
\end{tabular}

Tabela 8.19: Expression Template / Klein - Soma de vetor vezes escalar $(10 \leq n \leq 90)$

A partir de 300 elementos a Klein começa a ter rendimento melhor que a técnica expression template para soma com muitos termos. 


\begin{tabular}{|c|c|c|c|c|c|}
\hline $\begin{array}{c}\text { Tempo Expression } \\
\text { Tempo Klein }\end{array}$ & \multicolumn{5}{|c|}{ Número de elementos } \\
\hline Função & $\mathbf{1 0 0}$ & $\mathbf{3 0 0}$ & $\mathbf{5 0 0}$ & $\mathbf{7 0 0}$ & $\mathbf{9 0 0}$ \\
\hline $\mathbf{8 . 1 0}$ & 0,38 & 0,60 & 0,68 & 0,71 & 0,72 \\
\hline $\mathbf{8 . 1 1}$ & 0,47 & 0,71 & 0,87 & 0,82 & 0,85 \\
\hline $\mathbf{8 . 1 2}$ & 0,58 & 0,87 & 0,97 & 0,95 & $\mathbf{1 , 0 0}$ \\
\hline $\mathbf{8 . 1 3}$ & 0,70 & 0,95 & $\mathbf{1 , 0 1}$ & $\mathbf{1 , 0 5}$ & $\mathbf{1 , 1 0}$ \\
\hline $\mathbf{8 . 1 4}$ & 0,76 & $\mathbf{1 , 0 3}$ & $\mathbf{1 , 0 6}$ & $\mathbf{1 , 1 6}$ & $\mathbf{1 , 1 7}$ \\
\hline $\mathbf{8 . 1 5}$ & 0,85 & $\mathbf{1 , 0 6}$ & $\mathbf{1 , 1 0}$ & $\mathbf{1 , 2 1}$ & $\mathbf{1 , 2 3}$ \\
\hline $\mathbf{8 . 1 6}$ & 0,92 & $\mathbf{1 , 1 5}$ & $\mathbf{1 , 2 0}$ & $\mathbf{1 , 2 7}$ & $\mathbf{1 , 2 8}$ \\
\hline $\mathbf{8 . 1 7}$ & 0,87 & $\mathbf{1 , 0 9}$ & $\mathbf{1 , 1 6}$ & $\mathbf{1 , 1 6}$ & $\mathbf{1 , 1 7}$ \\
\hline $\mathbf{8 . 1 8}$ & 0,86 & $\mathbf{1 , 0 6}$ & $\mathbf{1 , 1 8}$ & $\mathbf{1 , 1 7}$ & $\mathbf{1 , 1 8}$ \\
\hline
\end{tabular}

Tabela 8.20: Expression Template / Klein - Soma de vetor vezes escalar $(100 \leq n \leq 900)$

Essa diferença de desempenho fica mais evidente a medida que aumentamos o número de elementos dos vetores.

\begin{tabular}{|c|c|c|c|c|c|}
\hline$\frac{5}{\text { Tempo Expression }}$ & \multicolumn{5}{|c|}{ Número de elementos } \\
\hline Tempo Klein & $\mathbf{1 0 0 0}$ & $\mathbf{3 0 0 0}$ & $\mathbf{5 0 0 0}$ & $\mathbf{7 0 0 0}$ & $\mathbf{9 0 0 0}$ \\
\hline Função & 0,71 & 0,72 & 0,72 & 0,71 & 0,71 \\
\hline $\mathbf{8 . 1 0}$ & 0,85 & 0,90 & 0,90 & 0,88 & 0,86 \\
\hline $\mathbf{8 . 1 1}$ & $\mathbf{1 , 0 1}$ & $\mathbf{1 , 0 2}$ & $\mathbf{1 , 0 2}$ & 0,98 & 0,98 \\
\hline $\mathbf{8 . 1 2}$ & $\mathbf{1 , 1 1}$ & $\mathbf{1 , 1 3}$ & $\mathbf{1 , 1 1}$ & $\mathbf{1 , 0 8}$ & $\mathbf{1 , 0 8}$ \\
\hline $\mathbf{8 . 1 3}$ & $\mathbf{1 , 1 7}$ & $\mathbf{1 , 1 9}$ & $\mathbf{1 , 1 6}$ & $\mathbf{1 , 1 5}$ & $\mathbf{1 , 1 5}$ \\
\hline $\mathbf{8 . 1 4}$ & $\mathbf{1 , 2 2}$ & $\mathbf{1 , 2 5}$ & $\mathbf{1 , 2 3}$ & $\mathbf{1 , 2 3}$ & $\mathbf{1 , 2 1}$ \\
\hline $\mathbf{8 . 1 5}$ & $\mathbf{1 , 2 8}$ & $\mathbf{1 , 3 0}$ & $\mathbf{1 , 2 8}$ & $\mathbf{1 , 2 7}$ & $\mathbf{1 , 2 7}$ \\
\hline $\mathbf{8 . 1 6}$ & $\mathbf{1 , 1 8}$ & $\mathbf{1 , 2 0}$ & $\mathbf{1 , 1 9}$ & $\mathbf{1 , 1 9}$ & $\mathbf{1 , 1 8}$ \\
\hline $\mathbf{8 . 1 7}$ & $\mathbf{1 , 1 8}$ & $\mathbf{1 , 1 9}$ & $\mathbf{1 , 1 9}$ & $\mathbf{1 , 1 8}$ & $\mathbf{1 , 1 8}$ \\
\hline $\mathbf{8 . 1 8}$ & & \multicolumn{5}{|c}{} \\
\hline
\end{tabular}

Tabela 8.21: Expression Template / Klein - Soma de vetor vezes escalar $(1000 \leq n \leq 9000)$

\section{Dummy / Klein}

A estratégia dummy é superior à Klein quando testamos vetores pequenos, porém para vetores grandes ela perde em todos os casos. 


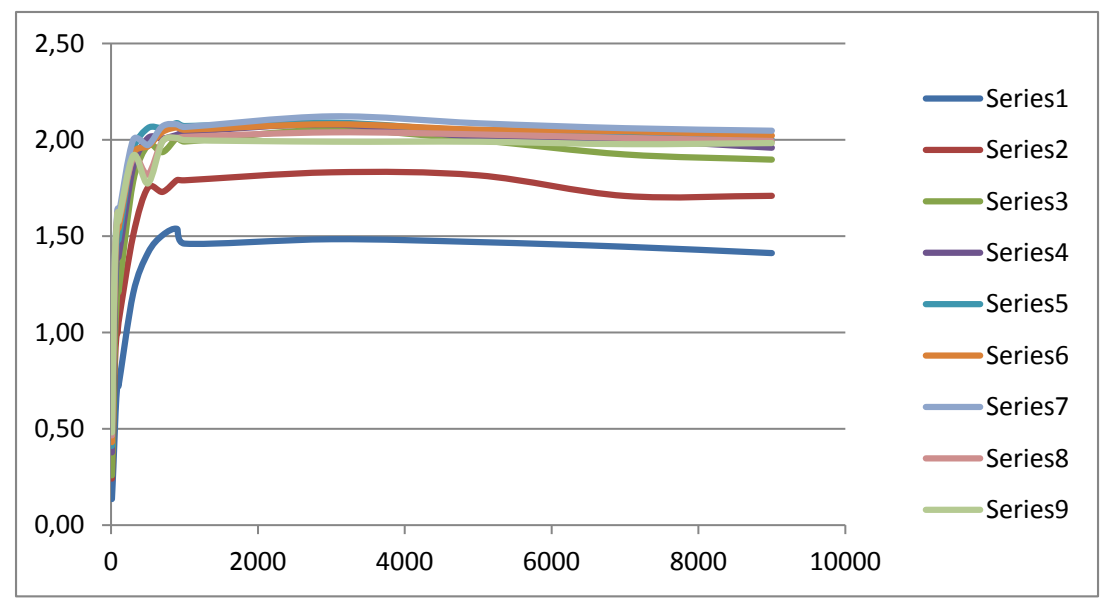

Figura 8.8: Multiplica escalar e soma vetores - Dummy / Klein

Quando efetuamos a operação para o algoritmo dummy notamos que para vetores entre 10 e 30 elementos ele é melhor que a Klein. Porém isso se inverte a medida que aumentamos o número de elementos dos vetores.

\begin{tabular}{|c|c|c|c|c|c|}
\hline $\begin{array}{c}\text { Tempo Dummy } \\
\text { Tempo Klein }\end{array}$ & \multicolumn{5}{|c|}{ Número de elementos } \\
\hline Função & $\mathbf{1 0}$ & $\mathbf{3 0}$ & $\mathbf{5 0}$ & $\mathbf{7 0}$ & $\mathbf{9 0}$ \\
\hline $\mathbf{8 . 1 0}$ & 0,14 & 0,30 & 0,49 & 0,66 & 0,74 \\
\hline $\mathbf{8 . 1 1}$ & 0,24 & 0,52 & 0,83 & 0,97 & $\mathbf{1 , 0 0}$ \\
\hline $\mathbf{8 . 1 2}$ & 0,26 & 0,54 & 0,95 & $\mathbf{1 , 1 2}$ & $\mathbf{1 , 2 5}$ \\
\hline $\mathbf{8 . 1 3}$ & 0,38 & 0,77 & $\mathbf{1 , 1 6}$ & $\mathbf{1 , 3 6}$ & $\mathbf{1 , 4 6}$ \\
\hline $\mathbf{8 . 1 4}$ & 0,41 & 0,85 & $\mathbf{1 , 2 3}$ & $\mathbf{1 , 4 4}$ & $\mathbf{1 , 5 4}$ \\
\hline $\mathbf{8 . 1 5}$ & 0,43 & 0,88 & $\mathbf{1 , 2 8}$ & $\mathbf{1 , 5 1}$ & $\mathbf{1 , 5 7}$ \\
\hline $\mathbf{8 . 1 6}$ & 0,48 & 0,95 & $\mathbf{1 , 3 8}$ & $\mathbf{1 , 5 5}$ & $\mathbf{1 , 6 5}$ \\
\hline $\mathbf{8 . 1 7}$ & 0,47 & 0,91 & $\mathbf{1 , 3 5}$ & $\mathbf{1 , 5 2}$ & $\mathbf{1 , 6 3}$ \\
\hline $\mathbf{8 . 1 8}$ & 0,48 & $\mathbf{1 , 0 0}$ & $\mathbf{1 , 3 9}$ & $\mathbf{1 , 5 4}$ & $\mathbf{1 , 6 3}$ \\
\hline
\end{tabular}

Tabela 8.22: Dummy / Klein - Soma de vetor vezes escalar $(10 \leq n \leq 90)$

Quando chegamos a 100 elementos a Klein passa a ganhar em todos os casos. Vale salientar, que diferente do algoritmo dummy soma de vetores, a soma de vetor vezes escalar para o caso de apenas dois vetores não é a estratégia utilizada na Klein. Isto porque ele resolve a multiplicação de cada vetor por um escalar individualmente e soma de forma inplace no vetor destino. Enquanto a estratégia da Klein é a estratégia expression template. 


\begin{tabular}{|c|c|c|c|c|c|}
\hline $\begin{array}{c}\text { Tempo Dummy } \\
\text { Tempo Klein }\end{array}$ & \multicolumn{4}{|c|}{ Número de elementos } \\
\hline Função & 100 & 300 & 500 & 700 & $\mathbf{9 0 0}$ \\
\hline 8.10 & 0,72 & 1,20 & 1,41 & 1,50 & 1,54 \\
\hline 8.11 & 1,05 & 1,50 & 1,75 & 1,73 & 1,79 \\
\hline 8.12 & 1,21 & 1,79 & 1,97 & 1,94 & 2,01 \\
\hline 8.13 & 1,40 & 1,86 & 2,01 & 2,01 & 2,03 \\
\hline 8.14 & 1,48 & 1,95 & 2,06 & 2,06 & 2,09 \\
\hline 8.15 & 1,54 & 1,93 & 1,97 & 2,04 & 2,06 \\
\hline 8.16 & 1,61 & 2,00 & 1,97 & 2,07 & 2,08 \\
\hline 8.17 & 1,58 & 1,90 & 1,82 & 1,99 & 2,01 \\
\hline 8.18 & 1,58 & 1,92 & 1,77 & 1,99 & 2,01 \\
\hline
\end{tabular}

Tabela 8.23: Dummy / Klein - Soma de vetor vezes escalar $(100 \leq n \leq 900)$

Quando a quantidade de elementos está entre 1000 e 9000 a vantagem da Klein se mantém.

\begin{tabular}{|c|c|c|c|c|c|}
\hline $\begin{array}{c}\text { Tempo Dummy } \\
\text { Tempo Klein }\end{array}$ & \multicolumn{5}{|c|}{ Número de elementos } \\
\hline Função & 1000 & 3000 & 5000 & 7000 & $\mathbf{9 0 0 0}$ \\
\hline 8.10 & 1,46 & 1,48 & 1,47 & 1,44 & 1,41 \\
\hline 8.11 & 1,79 & 1,83 & 1,82 & 1,71 & 1,71 \\
\hline 8.12 & 1,99 & 2,05 & 2,00 & 1,92 & 1,90 \\
\hline 8.13 & 2,04 & 2,08 & 2,02 & 2,00 & 1,96 \\
\hline 8.14 & 2,07 & 2,09 & 2,04 & 2,03 & 2,00 \\
\hline 8.15 & 2,05 & 2,08 & 2,05 & 2,05 & 2,02 \\
\hline 8.16 & 2,07 & 2,12 & 2,09 & 2,06 & 2,05 \\
\hline 8.17 & 2,02 & 2,04 & 2,03 & 2,01 & 1,99 \\
\hline 8.18 & 2,00 & 1,99 & 1,99 & 1,98 & 1,98 \\
\hline
\end{tabular}

Tabela 8.24: Dummy / Klein - Soma de vetor vezes escalar $(1000 \leq n \leq 9000)$

\subsection{Soma e subtração de vetor vezes escalar}

Para nos aproximar mais de uma expressão real, realizamos testes com soma e subtração de vetores.

$$
\begin{gathered}
r e s p=k_{1} * a-k_{2} * b \\
r e s p=k_{1} * a-k_{2} * b+k_{3} * c, \\
r e s p=k_{1} * a+k_{2} * b-k_{3} * c+k_{4} * d, \\
r e s p=k_{1} * a-k_{2} * b+k_{3} * c+k_{4} * d-k_{5} * e \\
r e s p=-k_{1} * a+k_{2} * b-k_{3} * c+k_{4} * d-k_{5} * e+k_{6} * f \\
r e s p=k_{1} * a-k_{2} * b+k_{3} * c-k_{4} * d+k_{5} * e-k_{6} * f-k_{7} * g, \\
r e s p=-k_{1} * a+k_{2} * b-k_{3} * c-k_{4} * d+k_{5} * e-k_{6} * f+k_{7} * g-k_{8} * h, \\
r e s p=k_{1} * a-k_{2} * b-k_{3} * c+k_{4} * d+k_{5} * e-k_{6} * f-k_{7} * g+k_{8} * h+k_{9} * i, \\
r e s p=-k_{1} * a-k_{2} * b+k_{3} * c-k_{4} * d+k_{5} * e-k_{6} * f+k_{7} * g+k_{8} * h-k_{9} * i-k_{10} * j,
\end{gathered}
$$

onde resp, $a, b, c, d, e, f, g, h, i$ e $j$ são vetores, e $k_{1}, k_{2}, k_{3}, k_{4}, k_{5}, k_{6}, k_{7}, k_{8}, k_{9}$ e $k_{10}$ são escalares.

Os códigos das bibliotecas MKL e ATLAS são iguais: 


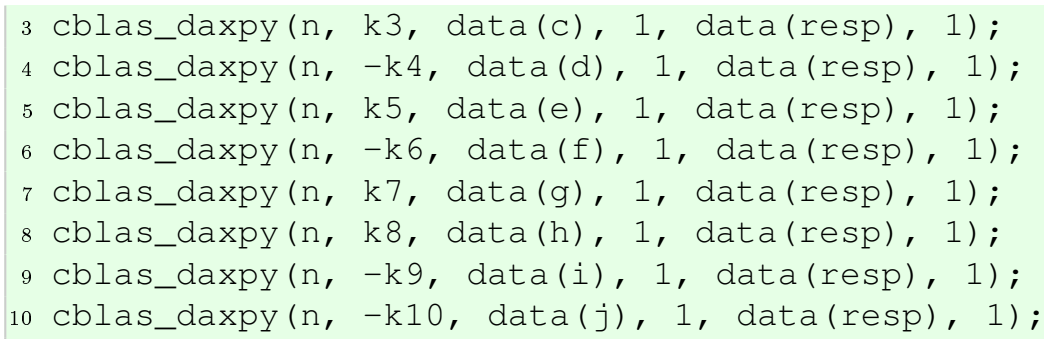

A ténica expression template foi implementada com um laço nos elementos dos vetores:

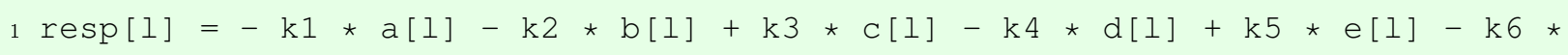

O código dummy fica dessa maneira:

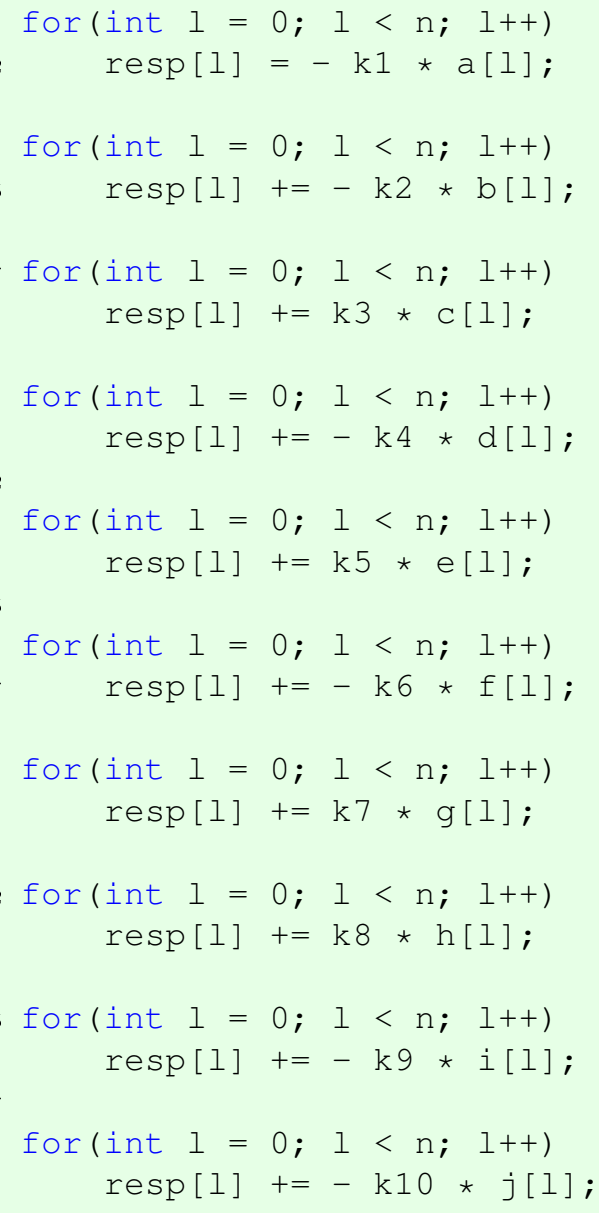

E o código da Klein, dessa maneira:

1 resp $=-\mathrm{k} 1$ * $\mathrm{a}-\mathrm{k} 2 \star \mathrm{b}+\mathrm{k} 3 \star \mathrm{c}-\mathrm{k} 4 \star \mathrm{d}+\mathrm{k} 5 \star \mathrm{e}-\mathrm{k} 6 \star \mathrm{f}+\mathrm{k} 7 \star \mathrm{g}+\mathrm{k} 8$ * $\mathrm{h}-\mathrm{k} 9 \star \mathrm{i}-\mathrm{k} 10 \star j ;$

\section{Atlas / Klein}

Para soma e subtração de vetores a biblioteca ATLAS possui rendimento melhor para vetores com poucos elementos. 


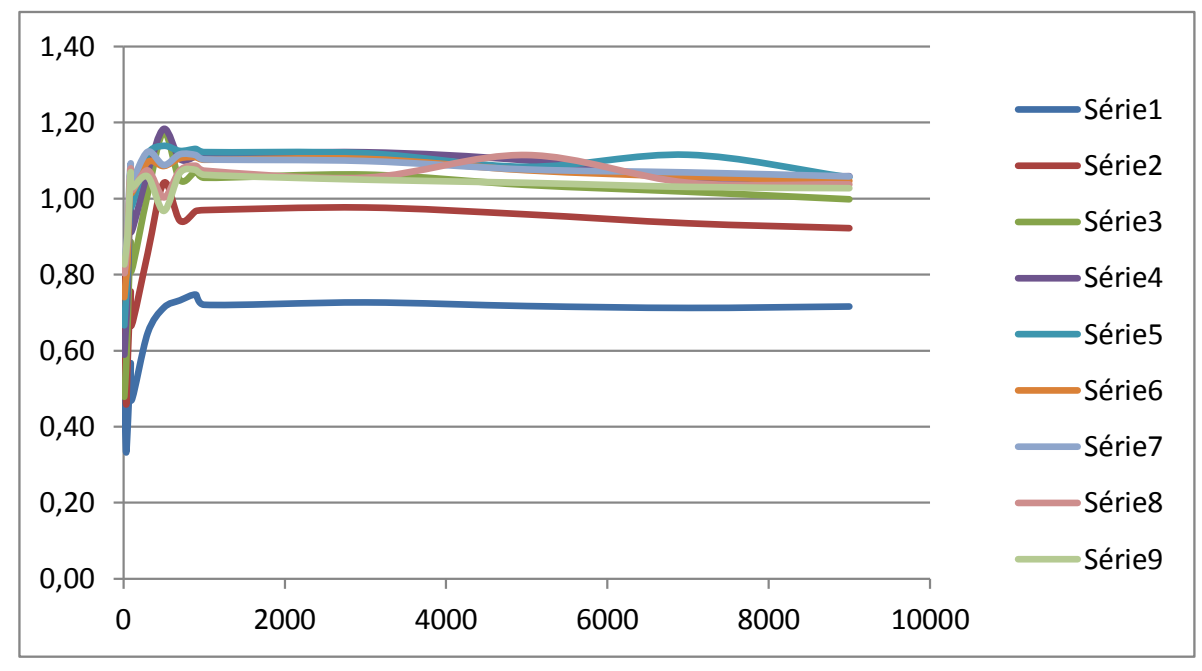

Figura 8.9: Multiplica escalar e soma vetores - Atlas / Klein

Para vetores entre 10 e 90 elementos, a medida que aumentamos a quantidade de elementos e de operações a Klein começa a ser mais competitiva.

\begin{tabular}{|c|c|c|c|c|c|}
\hline $\begin{array}{c}\text { Tempo Atlas } \\
\text { Tempo Klein }\end{array}$ & \multicolumn{5}{|c|}{ Número de elementos } \\
\hline Função & $\mathbf{1 0}$ & $\mathbf{3 0}$ & $\mathbf{5 0}$ & $\mathbf{7 0}$ & $\mathbf{9 0}$ \\
\hline $\mathbf{8 . 1 9}$ & 0,50 & 0,32 & 0,34 & 0,43 & 0,49 \\
\hline $\mathbf{8 . 2 0}$ & 0,80 & 0,44 & 0,51 & 0,62 & 0,69 \\
\hline $\mathbf{8 . 2 1}$ & 0,48 & 0,54 & 0,62 & 0,74 & 0,83 \\
\hline $\mathbf{8 . 2 2}$ & 0,60 & 0,66 & 0,74 & 0,91 & 0,98 \\
\hline $\mathbf{8 . 2 3}$ & 0,68 & 0,74 & 0,81 & 0,96 & $\mathbf{1 , 0 3}$ \\
\hline $\mathbf{8 . 2 4}$ & 0,74 & 0,80 & 0,84 & $\mathbf{1 , 0 0}$ & $\mathbf{1 , 0 5}$ \\
\hline $\mathbf{8 . 2 5}$ & 0,82 & 0,86 & 0,90 & $\mathbf{1 , 0 2}$ & $\mathbf{1 , 0 9}$ \\
\hline $\mathbf{8 . 2 6}$ & 0,80 & 0,83 & 0,90 & $\mathbf{1 , 0 1}$ & $\mathbf{1 , 0 8}$ \\
\hline $\mathbf{8 . 2 7}$ & 0,82 & 0,86 & 0,91 & 0,99 & $\mathbf{1 , 0 6}$ \\
\hline
\end{tabular}

Tabela 8.25: Atlas / Klein - Soma e subtração de vetor vezes escalar $(10 \leq n \leq 90)$

Quando analisamos vetores entre 100 e 900 elementos, a ATLAS se mantém mais rápida que a Klein para operações com apenas dois vetores. Porém a partir de 500 elementos a Klein torna-se mais rápida nos outros casos. 


\begin{tabular}{|c|c|c|c|c|c|}
\hline $\begin{array}{c}\text { Tempo Atlas } \\
\text { Tempo Klein }\end{array}$ & \multicolumn{5}{|c|}{ Número de elementos } \\
\hline Função & $\mathbf{1 0 0}$ & $\mathbf{3 0 0}$ & $\mathbf{5 0 0}$ & $\mathbf{7 0 0}$ & $\mathbf{9 0 0}$ \\
\hline $\mathbf{8 . 1 9}$ & 0,55 & 0,79 & 0,90 & 0,94 & 0,95 \\
\hline $\mathbf{8 . 2 0}$ & 0,73 & 0,91 & $\mathbf{1 , 0 4}$ & $\mathbf{1 , 0 1}$ & $\mathbf{1 , 0 4}$ \\
\hline $\mathbf{8 . 2 1}$ & 0,85 & $\mathbf{1 , 0 6}$ & $\mathbf{1 , 1 7}$ & $\mathbf{1 , 1 0}$ & $\mathbf{1 , 1 3}$ \\
\hline $\mathbf{8 . 2 2}$ & 0,91 & $\mathbf{1 , 0 6}$ & $\mathbf{1 , 1 8}$ & $\mathbf{1 , 1 1}$ & $\mathbf{1 , 1 1}$ \\
\hline $\mathbf{8 . 2 3}$ & 0,97 & $\mathbf{1 , 1 1}$ & $\mathbf{1 , 1 4}$ & $\mathbf{1 , 1 3}$ & $\mathbf{1 , 1 3}$ \\
\hline $\mathbf{8 . 2 4}$ & $\mathbf{1 , 0 1}$ & $\mathbf{1 , 1 0}$ & $\mathbf{1 , 0 8}$ & $\mathbf{1 , 1 1}$ & $\mathbf{1 , 1 1}$ \\
\hline $\mathbf{8 . 2 5}$ & $\mathbf{1 , 0 4}$ & $\mathbf{1 , 1 2}$ & $\mathbf{1 , 0 8}$ & $\mathbf{1 , 1 1}$ & $\mathbf{1 , 1 1}$ \\
\hline $\mathbf{8 . 2 6}$ & $\mathbf{1 , 0 2}$ & $\mathbf{1 , 0 7}$ & $\mathbf{1 , 0 0}$ & $\mathbf{1 , 0 8}$ & $\mathbf{1 , 0 8}$ \\
\hline $\mathbf{8 . 2 7}$ & $\mathbf{1 , 0 3}$ & $\mathbf{1 , 0 7}$ & 0,96 & $\mathbf{1 , 0 7}$ & $\mathbf{1 , 0 7}$ \\
\hline
\end{tabular}

Tabela 8.26: Atlas / Klein - Soma e subtração de vetor vezes escalar $(100 \leq n \leq 900)$

Para vetores entre 1000 e 9000 elementos, a Klein continua tendo bom desempenho, visto que perde em operações com dois ou três vetores, mas ganha em todas as outras expressões.

\begin{tabular}{|c|c|c|c|c|c|}
\hline $\begin{array}{c}\text { Tempo Atlas } \\
\text { Tempo Klein }\end{array}$ & \multicolumn{5}{|c|}{ Número de elementos } \\
\hline Função & $\mathbf{1 0 0 0}$ & $\mathbf{3 0 0 0}$ & $\mathbf{5 0 0 0}$ & $\mathbf{7 0 0 0}$ & $\mathbf{9 0 0 0}$ \\
\hline $\mathbf{8 . 1 9}$ & 0,72 & 0,73 & 0,90 & 0,89 & 0,87 \\
\hline $\mathbf{8 . 2 0}$ & 0,97 & 0,98 & $\mathbf{1 , 0 3}$ & 0,98 & 0,95 \\
\hline $\mathbf{8 . 2 1}$ & $\mathbf{1 , 0 5}$ & $\mathbf{1 , 0 6}$ & $\mathbf{1 , 1 0}$ & $\mathbf{1 , 0 6}$ & $\mathbf{1 , 0 3}$ \\
\hline $\mathbf{8 . 2 2}$ & $\mathbf{1 , 1 2}$ & $\mathbf{1 , 1 2}$ & $\mathbf{1 , 0 9}$ & $\mathbf{1 , 0 6}$ & $\mathbf{1 , 0 5}$ \\
\hline $\mathbf{8 . 2 3}$ & $\mathbf{1 , 1 2}$ & $\mathbf{1 , 1 2}$ & $\mathbf{1 , 0 7}$ & $\mathbf{1 , 1 1}$ & $\mathbf{1 , 0 6}$ \\
\hline $\mathbf{8 . 2 4}$ & $\mathbf{1 , 1 0}$ & $\mathbf{1 , 1 0}$ & $\mathbf{1 , 0 7}$ & $\mathbf{1 , 0 6}$ & $\mathbf{1 , 0 5}$ \\
\hline $\mathbf{8 . 2 5}$ & $\mathbf{1 , 1 0}$ & $\mathbf{1 , 1 0}$ & $\mathbf{1 , 0 7}$ & $\mathbf{1 , 0 6}$ & $\mathbf{1 , 0 6}$ \\
\hline $\mathbf{8 . 2 6}$ & $\mathbf{1 , 0 7}$ & $\mathbf{1 , 0 6}$ & $\mathbf{1 , 1 3}$ & $\mathbf{1 , 0 4}$ & $\mathbf{1 , 0 4}$ \\
\hline $\mathbf{8 . 2 7}$ & $\mathbf{1 , 0 6}$ & $\mathbf{1 , 0 5}$ & $\mathbf{1 , 0 4}$ & $\mathbf{1 , 0 3}$ & $\mathbf{1 , 0 3}$ \\
\hline
\end{tabular}

Tabela 8.27: Atlas / Klein - Soma e subtração de vetor vezes escalar $(1000 \leq n \leq 9000)$

MKL / Klein

Nesse caso, a Klein é inferior à MKL em quase todos os casos. 


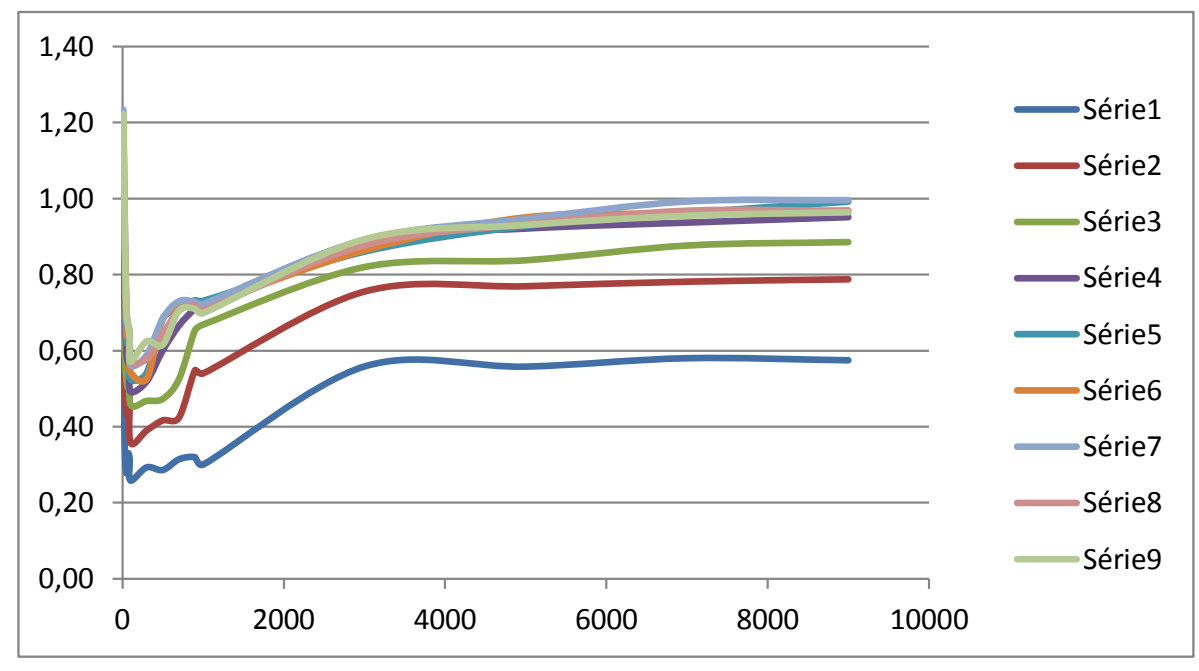

Figura 8.10: Multiplica escalar, soma e subtrai vetores - MKL / Klein

Quando comparamos os tempos da Klein e da MKL para vetores entre 10 e 90 elementos, percebemos que a Klein é competitiva para vetores com 10 elementos e a diferença vai aumentando a medida que o número de elementos aumenta.

\begin{tabular}{|c|c|c|c|c|c|}
\hline $\begin{array}{c}\text { Tempo MKL } \\
\text { Tempo Klein }\end{array}$ & \multicolumn{5}{|c|}{ Número de elementos } \\
\hline Função & $\mathbf{1 0}$ & $\mathbf{3 0}$ & $\mathbf{5 0}$ & $\mathbf{7 0}$ & $\mathbf{9 0}$ \\
\hline $\mathbf{8 . 1 9}$ & 0,64 & 0,34 & 0,27 & 0,28 & 0,28 \\
\hline $\mathbf{8 . 2 0}$ & 0,58 & 0,47 & 0,39 & 0,39 & 0,44 \\
\hline $\mathbf{8 . 2 1}$ & 0,74 & 0,58 & 0,48 & 0,46 & 0,53 \\
\hline $\mathbf{8 . 2 2}$ & 0,90 & 0,71 & 0,58 & 0,57 & 0,56 \\
\hline $\mathbf{8 . 2 3}$ & $\mathbf{1 , 0 2}$ & 0,79 & 0,62 & 0,63 & 0,61 \\
\hline $\mathbf{8 . 2 4}$ & $\mathbf{1 , 1 1}$ & 0,85 & 0,65 & 0,66 & 0,60 \\
\hline $\mathbf{8 . 2 5}$ & $\mathbf{1 , 2 6}$ & 0,91 & 0,69 & 0,65 & 0,64 \\
\hline $\mathbf{8 . 2 6}$ & $\mathbf{1 , 2 2}$ & 0,90 & 0,70 & 0,67 & 0,65 \\
\hline $\mathbf{8 . 2 7}$ & $\mathbf{1 , 2 1}$ & 0,89 & 0,69 & 0,67 & 0,63 \\
\hline
\end{tabular}

Tabela 8.28: $M K L /$ Klein - Soma e subtração de vetor vezes escalar $(10 \leq n \leq 90)$

Porém quando comparamos os tempos para vetores entre 100 e 900 elementos ao aumentarmos a quantidade de elementos dos vetores o desempenho da Klein melhora. 


\begin{tabular}{|c|c|c|c|c|c|}
\hline $\begin{array}{c}\text { Tempo MKL } \\
\text { Tempo Klein }\end{array}$ & \multicolumn{5}{|c|}{ Número de elementos } \\
\hline Função & $\mathbf{1 0 0}$ & $\mathbf{3 0 0}$ & $\mathbf{5 0 0}$ & $\mathbf{7 0 0}$ & $\mathbf{9 0 0}$ \\
\hline $\mathbf{8 . 1 9}$ & 0,30 & 0,36 & 0,36 & 0,40 & 0,41 \\
\hline $\mathbf{8 . 2 0}$ & 0,39 & 0,42 & 0,41 & 0,45 & 0,59 \\
\hline $\mathbf{8 . 2 1}$ & 0,48 & 0,49 & 0,47 & 0,55 & 0,69 \\
\hline $\mathbf{8 . 2 2}$ & 0,49 & 0,52 & 0,60 & 0,67 & 0,71 \\
\hline $\mathbf{8 . 2 3}$ & 0,52 & 0,54 & 0,69 & 0,71 & 0,73 \\
\hline $\mathbf{8 . 2 4}$ & 0,54 & 0,53 & 0,68 & 0,71 & 0,72 \\
\hline $\mathbf{8 . 2 5}$ & 0,56 & 0,59 & 0,68 & 0,73 & 0,73 \\
\hline $\mathbf{8 . 2 6}$ & 0,57 & 0,58 & 0,64 & 0,71 & 0,72 \\
\hline $\mathbf{8 . 2 7}$ & 0,57 & 0,63 & 0,61 & 0,70 & 0,71 \\
\hline
\end{tabular}

Tabela 8.29: $M K L /$ Klein - Soma e subtração de vetor vezes escalar $(100 \leq n \leq 900)$

Esse comportamento se repete para vetores entre 1000 e 9000 elementos.

\begin{tabular}{|c|c|c|c|c|c|}
\hline Tempo MKL & \multicolumn{5}{|c|}{ Número de elementos } \\
\hline Tempo Klein & \multicolumn{5}{|c|}{} \\
\hline Função & $\mathbf{1 0 0 0}$ & $\mathbf{3 0 0 0}$ & $\mathbf{5 0 0 0}$ & $\mathbf{7 0 0 0}$ & $\mathbf{9 0 0 0}$ \\
\hline $\mathbf{8 . 1 9}$ & 0,30 & 0,56 & 0,70 & 0,72 & 0,70 \\
\hline $\mathbf{8 . 2 0}$ & 0,54 & 0,76 & 0,82 & 0,82 & 0,82 \\
\hline $\mathbf{8 . 2 1}$ & 0,67 & 0,82 & 0,89 & 0,91 & 0,91 \\
\hline $\mathbf{8 . 2 2}$ & 0,72 & 0,89 & 0,91 & 0,94 & 0,94 \\
\hline $\mathbf{8 . 2 3}$ & 0,73 & 0,86 & 0,92 & 0,96 & 0,99 \\
\hline $\mathbf{8 . 2 4}$ & 0,72 & 0,86 & 0,94 & 0,96 & 0,97 \\
\hline $\mathbf{8 . 2 5}$ & 0,72 & 0,89 & 0,94 & 0,99 & 0,99 \\
\hline $\mathbf{8 . 2 6}$ & 0,71 & 0,88 & 0,94 & 0,97 & 0,97 \\
\hline $\mathbf{8 . 2 7}$ & 0,70 & 0,89 & 0,93 & 0,95 & 0,96 \\
\hline
\end{tabular}

Tabela 8.30: $M K L /$ Klein - Soma e subtração de vetor vezes escalar $(1000 \leq n \leq 9000)$

\section{Expression template x Klein}

O resultado dessa comparação foi muito semelhante ao da soma vetores e multiplica escalar.

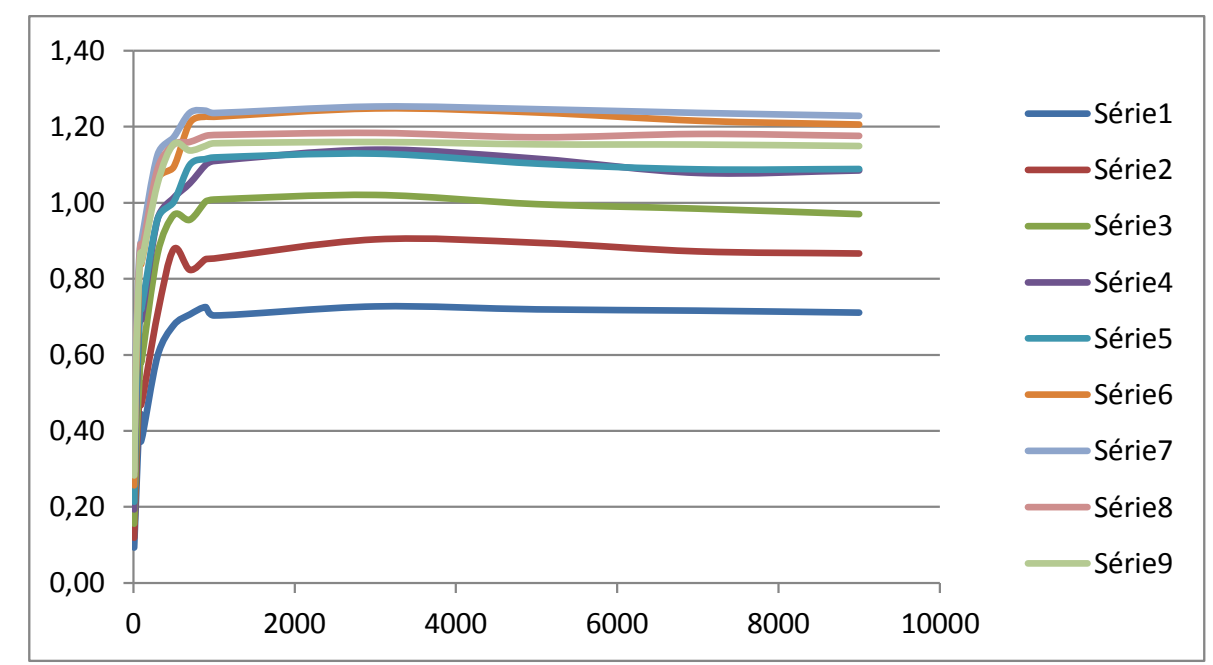

Figura 8.11: Multiplica escalar, soma e subtrai vetores - Expression / Klein 
A técnica expression template é mais rápida quando comparamos vetores entre 10 e 90 elementos. Porém podemos perceber que a medida que a quantidade de elementos aumenta a diferença entre elas diminui.

\begin{tabular}{|c|c|c|c|c|c|}
\hline $\begin{array}{c}\text { Tempo Expression } \\
\text { Tempo Klein }\end{array}$ & \multicolumn{5}{|c|}{ Número de elementos } \\
\hline Função & $\mathbf{1 0}$ & $\mathbf{3 0}$ & $\mathbf{5 0}$ & $\mathbf{7 0}$ & $\mathbf{9 0}$ \\
\hline $\mathbf{8 . 1 9}$ & 0,09 & 0,19 & 0,27 & 0,35 & 0,38 \\
\hline $\mathbf{8 . 2 0}$ & 0,12 & 0,24 & 0,35 & 0,43 & 0,48 \\
\hline $\mathbf{8 . 2 1}$ & 0,16 & 0,31 & 0,43 & 0,51 & 0,59 \\
\hline $\mathbf{8 . 2 2}$ & 0,19 & 0,43 & 0,55 & 0,66 & 0,73 \\
\hline $\mathbf{8 . 2 3}$ & 0,21 & 0,46 & 0,58 & 0,68 & 0,74 \\
\hline $\mathbf{8 . 2 4}$ & 0,26 & 0,53 & 0,65 & 0,81 & 0,88 \\
\hline $\mathbf{8 . 2 5}$ & 0,29 & 0,60 & 0,73 & 0,85 & 0,90 \\
\hline $\mathbf{8 . 2 6}$ & 0,30 & 0,57 & 0,72 & 0,83 & 0,89 \\
\hline $\mathbf{8 . 2 7}$ & 0,28 & 0,56 & 0,70 & 0,81 & 0,87 \\
\hline
\end{tabular}

Tabela 8.31: Expression Template / Klein - Soma e subtração de vetor vezes escalar $(10 \leq n \leq 90)$

Para vetores entre 100 e 900 elementos a Klein começa a se sair melhor a partir de 500 elementos, onde ganha na maioria dos casos, exceto os casos em que são efetuadas operações com até três vetores.

\begin{tabular}{|c|c|c|c|c|c|}
\hline$\frac{\mid}{\text { Tempo Expression }}$ & \multicolumn{5}{|c|}{ Número de elementos } \\
\hline Tempo Klein & \multicolumn{3}{|c|}{} \\
\hline Função & $\mathbf{1 0 0}$ & $\mathbf{3 0 0}$ & $\mathbf{5 0 0}$ & $\mathbf{7 0 0}$ & $\mathbf{9 0 0}$ \\
\hline $\mathbf{8 . 1 9}$ & 0,38 & 0,60 & 0,68 & 0,71 & 0,73 \\
\hline $\mathbf{8 . 2 0}$ & 0,47 & 0,71 & 0,88 & 0,82 & 0,85 \\
\hline $\mathbf{8 . 2 1}$ & 0,58 & 0,87 & 0,97 & 0,96 & $\mathbf{1 , 0 0}$ \\
\hline $\mathbf{8 . 2 2}$ & 0,69 & 0,96 & $\mathbf{1 , 0 1}$ & $\mathbf{1 , 0 5}$ & $\mathbf{1 , 1 0}$ \\
\hline $\mathbf{8 . 2 3}$ & 0,72 & 0,96 & $\mathbf{1 , 0 0}$ & $\mathbf{1 , 1 0}$ & $\mathbf{1 , 1 2}$ \\
\hline $\mathbf{8 . 2 4}$ & 0,84 & $\mathbf{1 , 0 6}$ & $\mathbf{1 , 0 9}$ & $\mathbf{1 , 2 1}$ & $\mathbf{1 , 2 3}$ \\
\hline $\mathbf{8 . 2 5}$ & 0,90 & $\mathbf{1 , 1 3}$ & $\mathbf{1 , 1 7}$ & $\mathbf{1 , 2 4}$ & $\mathbf{1 , 2 4}$ \\
\hline $\mathbf{8 . 2 6}$ & 0,87 & $\mathbf{1 , 0 9}$ & $\mathbf{1 , 1 5}$ & $\mathbf{1 , 1 6}$ & $\mathbf{1 , 1 8}$ \\
\hline $\mathbf{8 . 2 7}$ & 0,84 & $\mathbf{1 , 0 5}$ & $\mathbf{1 , 1 5}$ & $\mathbf{1 , 1 4}$ & $\mathbf{1 , 1 5}$ \\
\hline
\end{tabular}

Tabela 8.32: Expression Template / Klein - Soma e subtração de vetor vezes escalar $(100 \leq n \leq 900)$

Para vetores entre 1000 e 9000 elementos a estratégia expression template ganha em todos os casos em que existem apenas três vetores envolvidos, porém perde em todos os outros casos. 


\begin{tabular}{|c|c|c|c|c|c|}
\hline $\begin{array}{c}\text { Tempo Expression } \\
\text { Tempo Klein }\end{array}$ & \multicolumn{5}{|c|}{ Número de elementos } \\
\hline Função & $\mathbf{1 0 0 0}$ & $\mathbf{3 0 0 0}$ & $\mathbf{5 0 0 0}$ & $\mathbf{7 0 0 0}$ & $\mathbf{9 0 0 0}$ \\
\hline $\mathbf{8 . 1 9}$ & 0,70 & 0,73 & 0,72 & 0,72 & 0,71 \\
\hline $\mathbf{8 . 2 0}$ & 0,85 & 0,90 & 0,89 & 0,87 & 0,87 \\
\hline $\mathbf{8 . 2 1}$ & $\mathbf{1 , 0 1}$ & $\mathbf{1 , 0 2}$ & $\mathbf{1 , 0 5}$ & $\mathbf{1 , 0 2}$ & $\mathbf{1 , 0 0}$ \\
\hline $\mathbf{8 . 2 2}$ & $\mathbf{1 , 1 1}$ & $\mathbf{1 , 1 4}$ & $\mathbf{1 , 1 0}$ & $\mathbf{1 , 0 9}$ & $\mathbf{1 , 0 7}$ \\
\hline $\mathbf{8 . 2 3}$ & $\mathbf{1 , 1 2}$ & $\mathbf{1 , 1 3}$ & $\mathbf{1 , 0 9}$ & $\mathbf{1 , 0 9}$ & $\mathbf{1 , 0 9}$ \\
\hline $\mathbf{8 . 2 4}$ & $\mathbf{1 , 2 3}$ & $\mathbf{1 , 2 5}$ & $\mathbf{1 , 2 3}$ & $\mathbf{1 , 2 2}$ & $\mathbf{1 , 2 1}$ \\
\hline $\mathbf{8 . 2 5}$ & $\mathbf{1 , 2 4}$ & $\mathbf{1 , 2 5}$ & $\mathbf{1 , 2 4}$ & $\mathbf{1 , 2 3}$ & $\mathbf{1 , 2 2}$ \\
\hline $\mathbf{8 . 2 6}$ & $\mathbf{1 , 1 8}$ & $\mathbf{1 , 1 8}$ & $\mathbf{1 , 1 8}$ & $\mathbf{1 , 1 8}$ & $\mathbf{1 , 1 8}$ \\
\hline $\mathbf{8 . 2 7}$ & $\mathbf{1 , 1 6}$ & $\mathbf{1 , 1 6}$ & $\mathbf{1 , 1 5}$ & $\mathbf{1 , 1 5}$ & $\mathbf{1 , 1 5}$ \\
\hline
\end{tabular}

Tabela 8.33: Expression Template / Klein - Soma e subtração de vetor vezes escalar $(1000 \leq n \leq 9000)$

\section{Dummy / Klein}

Para vetores grandes a Klein só não é melhor para o caso em que efetuamos apenas uma operação.

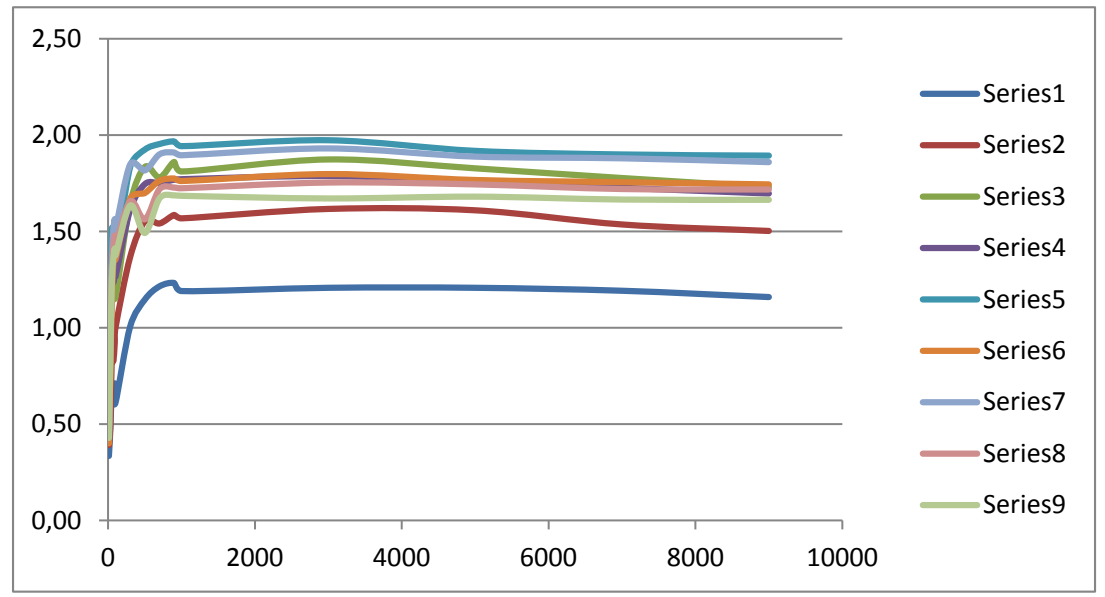

Figura 8.12: Multiplica escalar, soma e subtrai vetores - Dummy / Klein

A Klein começa a se sair melhor nos vetores a partir de 50 elementos.

\begin{tabular}{|c|c|c|c|c|c|}
\hline $\begin{array}{c}\text { Tempo Dummy } \\
\text { Tempo Klein }\end{array}$ & \multicolumn{5}{|c|}{ Número de elementos } \\
\hline Função & $\mathbf{1 0}$ & $\mathbf{3 0}$ & $\mathbf{5 0}$ & $\mathbf{7 0}$ & $\mathbf{9 0}$ \\
\hline $\mathbf{8 . 1 9}$ & 0,34 & 0,48 & 0,69 & 0,68 & 0,71 \\
\hline $\mathbf{8 . 2 0}$ & 0,39 & 0,50 & 0,86 & 0,83 & 0,93 \\
\hline $\mathbf{8 . 2 1}$ & 0,45 & 0,80 & $\mathbf{1 , 2 3}$ & $\mathbf{1 , 1 4}$ & $\mathbf{1 , 2 5}$ \\
\hline $\mathbf{8 . 2 2}$ & 0,43 & 0,90 & $\mathbf{1 , 3 4}$ & $\mathbf{1 , 2 3}$ & $\mathbf{1 , 3 2}$ \\
\hline $\mathbf{8 . 2 3}$ & 0,49 & $\mathbf{1 , 0 1}$ & $\mathbf{1 , 5 1}$ & $\mathbf{1 , 3 8}$ & $\mathbf{1 , 4 8}$ \\
\hline $\mathbf{8 . 2 4}$ & 0,52 & $\mathbf{1 , 0 4}$ & $\mathbf{1 , 1 9}$ & $\mathbf{1 , 3 6}$ & $\mathbf{1 , 3 9}$ \\
\hline $\mathbf{8 . 2 5}$ & 0,54 & $\mathbf{1 , 0 8}$ & $\mathbf{1 , 3 1}$ & $\mathbf{1 , 4 8}$ & $\mathbf{1 , 5 6}$ \\
\hline $\mathbf{8 . 2 6}$ & 0,52 & $\mathbf{1 , 0 6}$ & $\mathbf{1 , 2 5}$ & $\mathbf{1 , 3 7}$ & $\mathbf{1 , 4 5}$ \\
\hline $\mathbf{8 . 2 7}$ & 0,59 & $\mathbf{1 , 1 6}$ & $\mathbf{1 , 2 7}$ & $\mathbf{1 , 3 8}$ & $\mathbf{1 , 4 3}$ \\
\hline
\end{tabular}

Tabela 8.34: Dummy / Klein - Soma e subtração de vetor vezes escalar $(10 \leq n \leq 90)$ 
Quando a quantidade de elementos dos vetores chega a 300, a Klein é sempre superior que a estratégia dummy.

\begin{tabular}{|c|c|c|c|c|c|}
\hline $\begin{array}{c}\text { Tempo Dummy } \\
\text { Tempo Klein }\end{array}$ & \multicolumn{5}{|c|}{ Número de elementos } \\
\hline Função & 100 & 300 & 500 & 700 & $\mathbf{9 0 0}$ \\
\hline 8.19 & 0,69 & 1,03 & 1,18 & 1,24 & 1,25 \\
\hline 8.20 & 0,91 & 1,31 & 1,52 & 1,51 & 1,57 \\
\hline 8.21 & 1,20 & 1,67 & 1,84 & 1,79 & 1,86 \\
\hline 8.22 & 1,25 & 1,60 & 1,74 & 1,74 & 1,76 \\
\hline 8.23 & 1,42 & 1,84 & 1,94 & 1,95 & 1,97 \\
\hline 8.24 & 1,39 & 1,67 & 1,69 & 1,77 & 1,78 \\
\hline 8.25 & 1,52 & 1,90 & 1,88 & 1,98 & 1,99 \\
\hline 8.26 & 1,39 & 1,64 & 1,55 & 1,72 & 1,72 \\
\hline 8.27 & 1,39 & 1,62 & 1,48 & 1,69 & 1,69 \\
\hline
\end{tabular}

Tabela 8.35: Dummy / Klein - Soma e subtração de vetor vezes escalar $(100 \leq n \leq 900)$

Ao aumentar a quantidade de elementos até 3000 a Klein aumenta a vantagem, porém a partir daí tem uma sensível redução.

\begin{tabular}{|c|c|c|c|c|c|}
\hline $\begin{array}{c}\text { Tempo Dummy } \\
\text { Tempo Klein }\end{array}$ & \multicolumn{5}{|c|}{ Número de elementos } \\
\hline Função & 1000 & 3000 & 5000 & 7000 & $\mathbf{9 0 0 0}$ \\
\hline 8.19 & 1,21 & 1,21 & 1,22 & 1,21 & 1,19 \\
\hline 8.20 & 1,55 & 1,61 & 1,61 & 1,54 & 1,51 \\
\hline 8.21 & 1,82 & 1,88 & 1,83 & 1,78 & 1,73 \\
\hline 8.22 & 1,77 & 1,79 & 1,76 & 1,73 & 1,70 \\
\hline 8.23 & 1,95 & 1,98 & 1,92 & 1,90 & 1,89 \\
\hline 8.24 & 1,77 & 1,81 & 1,76 & 1,76 & 1,75 \\
\hline 8.25 & 1,98 & 2,02 & 1,97 & 1,96 & 1,95 \\
\hline 8.26 & 1,72 & 1,75 & 1,74 & 1,72 & 1,72 \\
\hline 8.27 & 1,68 & 1,67 & 1,68 & 1,68 & 1,66 \\
\hline
\end{tabular}

Tabela 8.36: Dummy / Klein - Soma e subtração de vetor vezes escalar (1000 $\leq n \leq 9000)$

\subsection{Soma de matrizes}

Realizamos os teste também para soma de matrizes.

$$
\begin{gathered}
r e s p=a+b, \\
r e s p=a+b+c, \\
r e s p=a+b+c+d, \\
r e s p=a+b+c+d+e, \\
r e s p=a+b+c+d+e+f, \\
r e s p=a+b+c+d+e+f+g, \\
r e s p=a+b+c+d+e+f+g+h, \\
r e s p=a+b+c+d+e+f+g+h+i, \\
r e s p=a+b+c+d+e+f+g+h+i+j,
\end{gathered}
$$


onde resp, $a, b, c, d, e, f, g, h, i$ e $j$ são matrizes,

O código da biblioteca MKL foi escrito dessa forma,

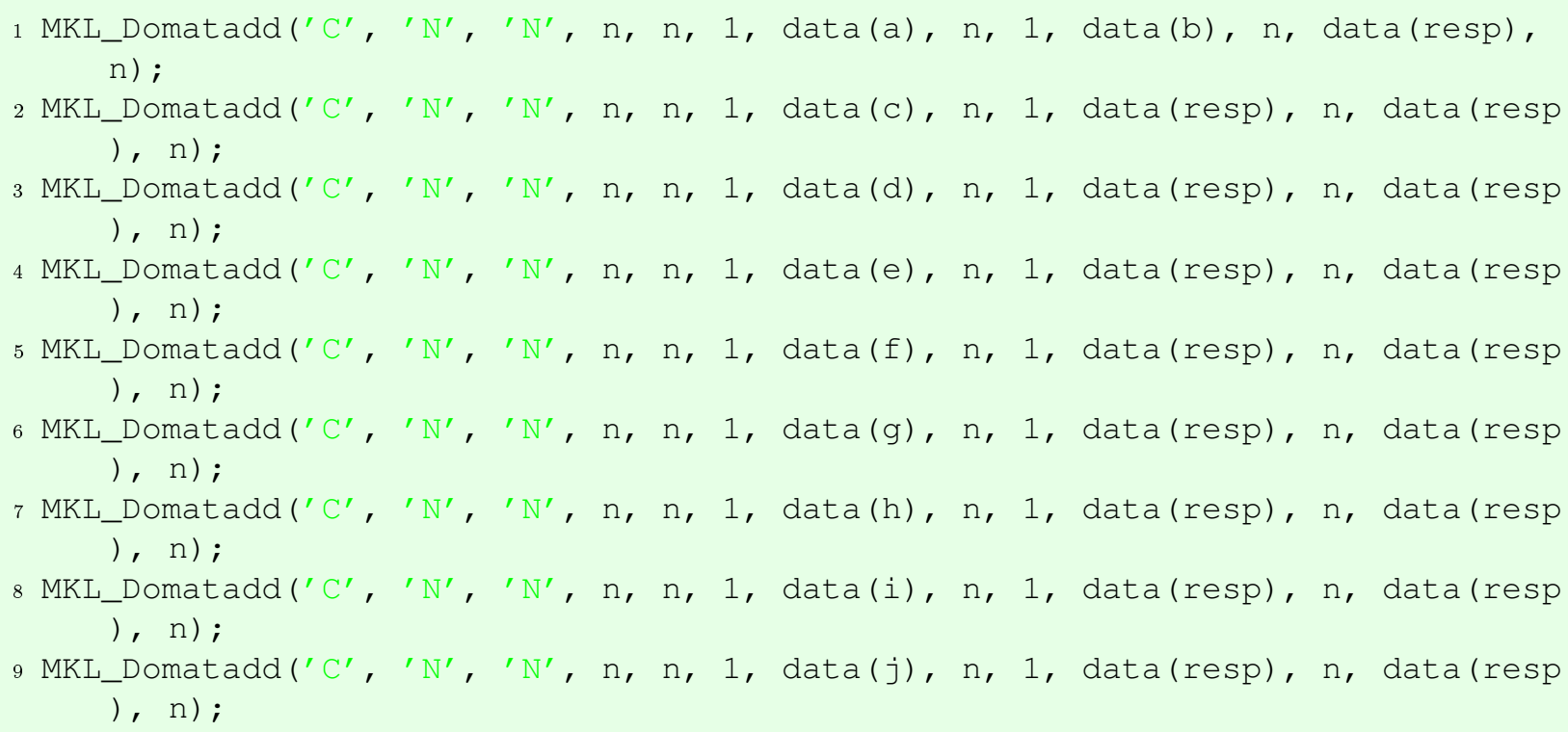

a técnica expression template foi implementada com dois laços:

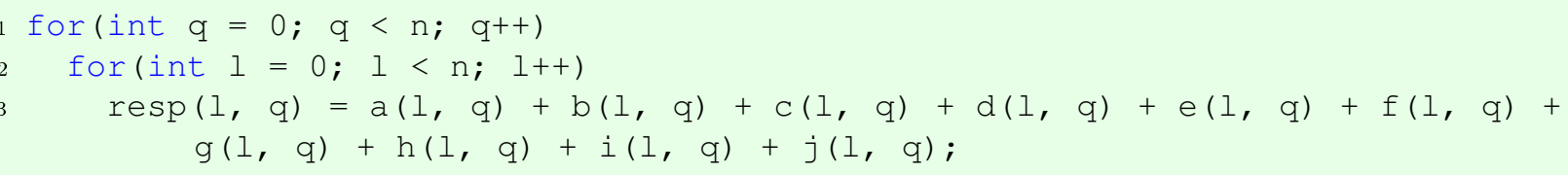

o código dummy fica dessa maneira:

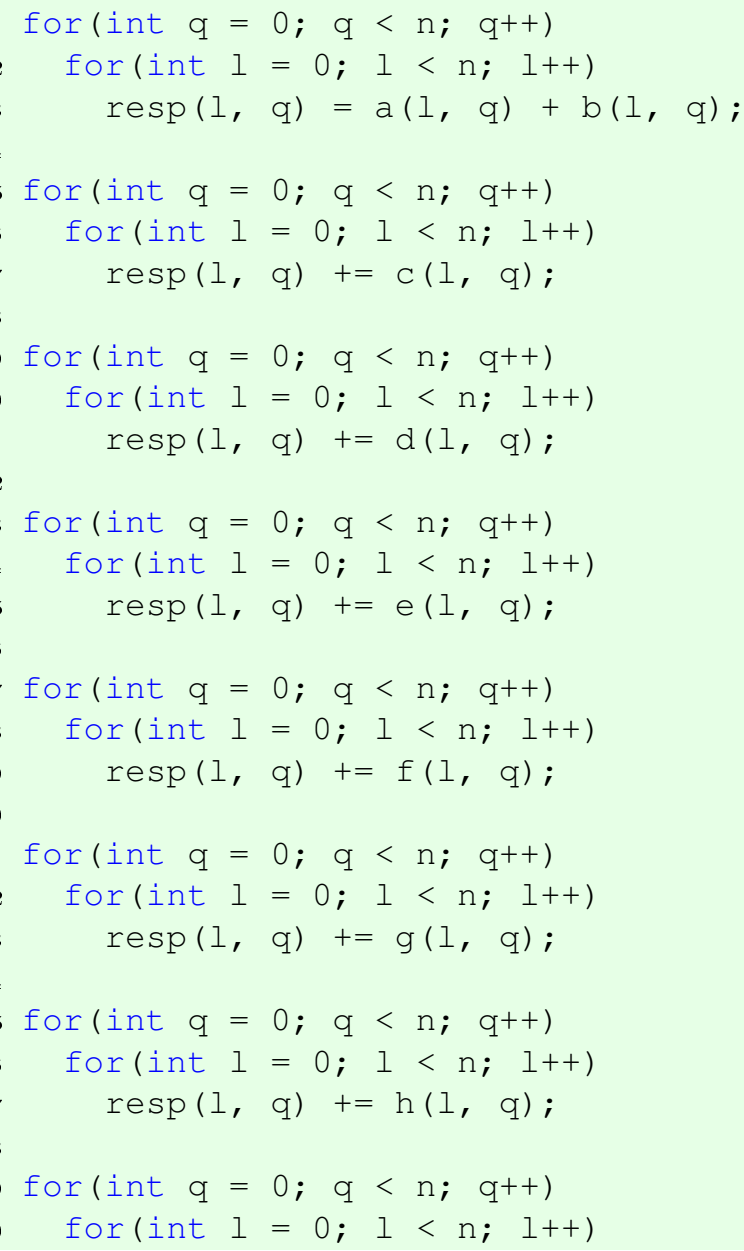




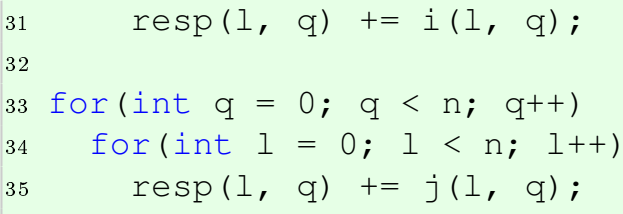

e a Klein intuitivamente,

$r \operatorname{sp}=a+b+c+d+e+f+g+h+i+j ;$

MKL / Klein

Para a soma de matrizes existe um intervalo razoável em que a Klein é superior à MKL.

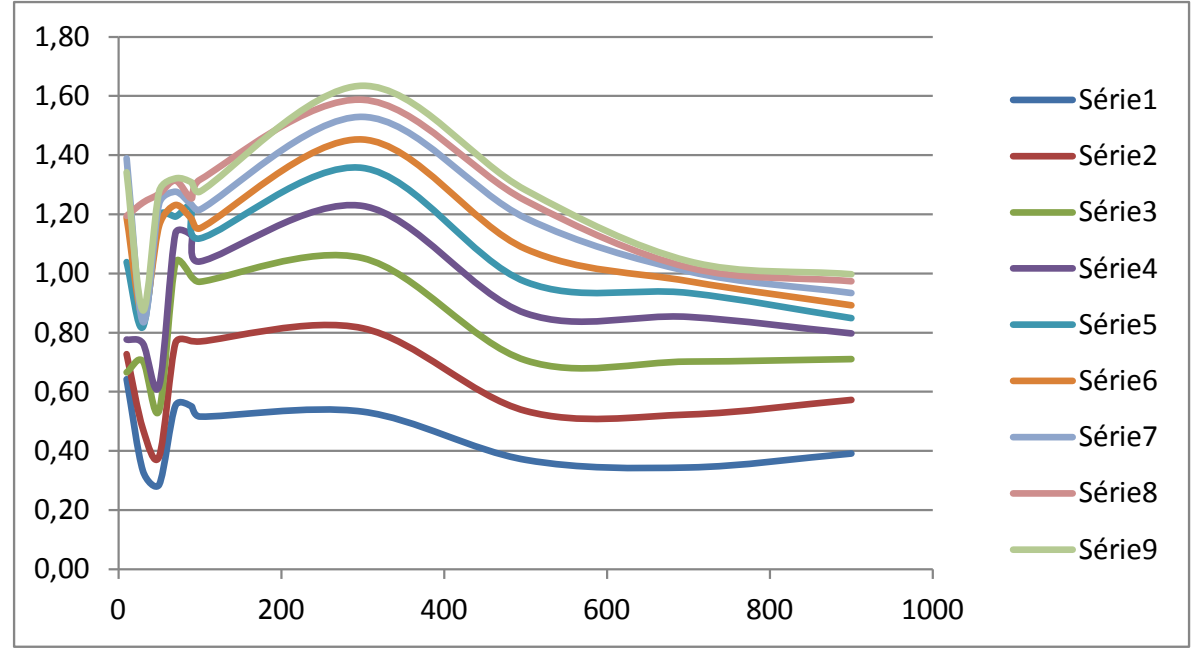

Figura 8.13: Soma de matrizes - MKL / Klein

Para matrizes de 10x10 até 90x90 elementos, quando somamos até três matrizes a biblioteca MKL é superior na maioria dos casos, porém para os outros casos a Klein é superior.

\begin{tabular}{|c|c|c|c|c|c|}
\hline $\begin{array}{c}\text { Tempo MKL } \\
\text { Tempo Klein }\end{array}$ & \multicolumn{5}{|c|}{ Número de elementos } \\
\hline Função & $\mathbf{1 0}$ & $\mathbf{3 0}$ & $\mathbf{5 0}$ & $\mathbf{7 0}$ & $\mathbf{9 0}$ \\
\hline $\mathbf{8 . 2 8}$ & 0,64 & 0,33 & 0,29 & 0,55 & 0,55 \\
\hline $\mathbf{8 . 2 9}$ & 0,73 & 0,47 & 0,38 & 0,77 & 0,77 \\
\hline $\mathbf{8 . 3 0}$ & 0,67 & 0,70 & 0,54 & $\mathbf{1 , 0 4}$ & 0,99 \\
\hline $\mathbf{8 . 3 1}$ & 0,78 & 0,76 & 0,62 & $\mathbf{1 , 1 4}$ & $\mathbf{1 , 1 3}$ \\
\hline $\mathbf{8 . 3 2}$ & $\mathbf{1 , 0 4}$ & 0,82 & $\mathbf{1 , 2 0}$ & $\mathbf{1 , 1 9}$ & $\mathbf{1 , 2 3}$ \\
\hline $\mathbf{8 . 3 3}$ & $\mathbf{1 , 1 9}$ & 0,86 & $\mathbf{1 , 1 6}$ & $\mathbf{1 , 2 3}$ & $\mathbf{1 , 1 8}$ \\
\hline $\mathbf{8 . 3 4}$ & $\mathbf{1 , 3 9}$ & 0,84 & $\mathbf{1 , 2 4}$ & $\mathbf{1 , 2 8}$ & $\mathbf{1 , 2 3}$ \\
\hline $\mathbf{8 . 3 5}$ & $\mathbf{1 , 1 9}$ & $\mathbf{1 , 2 4}$ & $\mathbf{1 , 2 7}$ & $\mathbf{1 , 3 1}$ & $\mathbf{1 , 2 5}$ \\
\hline $\mathbf{8 . 3 6}$ & $\mathbf{1 , 3 4}$ & 0,88 & $\mathbf{1 , 2 8}$ & $\mathbf{1 , 3 2}$ & $\mathbf{1 , 3 1}$ \\
\hline
\end{tabular}

Tabela 8.37: $M K L /$ Klein - Soma de matrizes $(10 \leq n \leq 90)$

Quando rodamos para matrizes entre 100x100 e 900x900 elementos percebemos que a medida que aumentamos o número de elementos das matrizes a MKL começa a ser superior. 


\begin{tabular}{|c|c|c|c|c|c|}
\hline $\begin{array}{c}\text { Tempo MKL } \\
\text { Tempo Klein }\end{array}$ & \multicolumn{5}{|c|}{ Número de elementos } \\
\hline Função & $\mathbf{1 0 0}$ & $\mathbf{3 0 0}$ & $\mathbf{5 0 0}$ & $\mathbf{7 0 0}$ & $\mathbf{9 0 0}$ \\
\hline $\mathbf{8 . 2 8}$ & 0,52 & 0,53 & 0,37 & 0,34 & 0,39 \\
\hline $\mathbf{8 . 2 9}$ & 0,77 & 0,81 & 0,54 & 0,52 & 0,57 \\
\hline $\mathbf{8 . 3 0}$ & 0,97 & $\mathbf{1 , 0 5}$ & 0,71 & 0,70 & 0,71 \\
\hline $\mathbf{8 . 3 1}$ & $\mathbf{1 , 0 4}$ & $\mathbf{1 , 2 3}$ & 0,87 & 0,85 & 0,80 \\
\hline $\mathbf{8 . 3 2}$ & $\mathbf{1 , 1 2}$ & $\mathbf{1 , 3 6}$ & 0,97 & 0,93 & 0,85 \\
\hline $\mathbf{8 . 3 3}$ & $\mathbf{1 , 1 5}$ & $\mathbf{1 , 4 5}$ & $\mathbf{1 , 0 8}$ & 0,97 & 0,89 \\
\hline $\mathbf{8 . 3 4}$ & $\mathbf{1 , 2 2}$ & $\mathbf{1 , 5 3}$ & $\mathbf{1 , 1 9}$ & $\mathbf{1 , 0 1}$ & 0,93 \\
\hline $\mathbf{8 . 3 5}$ & $\mathbf{1 , 3 2}$ & $\mathbf{1 , 5 9}$ & $\mathbf{1 , 2 5}$ & $\mathbf{1 , 0 2}$ & 0,97 \\
\hline $\mathbf{8 . 3 6}$ & $\mathbf{1 , 2 8}$ & $\mathbf{1 , 6 3}$ & $\mathbf{1 , 2 8}$ & $\mathbf{1 , 0 4}$ & $\mathbf{1 , 0 0}$ \\
\hline
\end{tabular}

Tabela 8.38: MKL / Klein - Soma de matrizes $(100 \leq n \leq 900)$

\section{Expression template $\mathrm{x}$ Klein}

Ao compararmos com a técnica expression template podemos dizer que nesse caso a Klein é inferior.

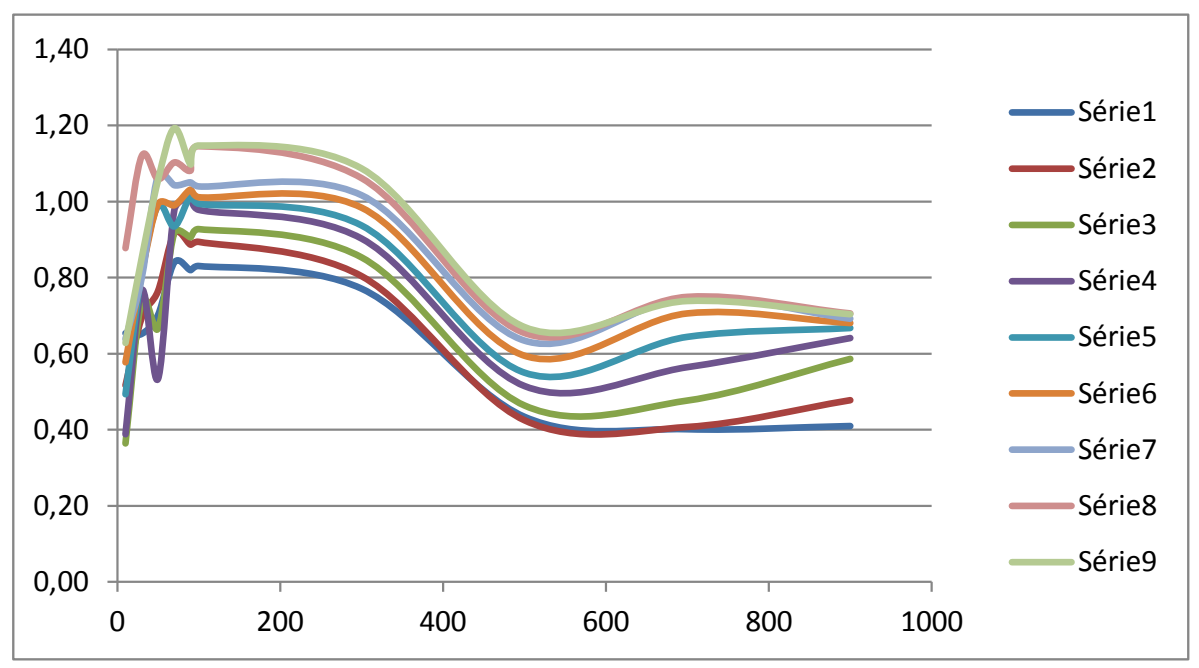

Figura 8.14: Soma de matrizes - Expression / Klein

\begin{tabular}{|c|c|c|c|c|c|}
\hline$\frac{\text { Tempo Expression }}{\text { Tempo Klein }}$ & \multicolumn{5}{|c|}{ Número de elementos } \\
\hline Função & 10 & 30 & 50 & 70 & 90 \\
\hline 8.28 & 0,65 & 0,65 & 0,70 & 0,84 & 0,82 \\
\hline 8.29 & 0,52 & 0,70 & 0,77 & 0,92 & 0,89 \\
\hline 8.30 & 0,36 & 0,74 & 0,67 & 0,92 & 0,91 \\
\hline 8.31 & 0,39 & 0,77 & 0,53 & 0,98 & 1,01 \\
\hline 8.32 & 0,49 & 0,81 & 0,99 & 0,93 & 1,02 \\
\hline 8.33 & 0,58 & 0,81 & 0,99 & 0,99 & 1,03 \\
\hline 8.34 & 0,64 & 0,80 & 1,07 & 1,04 & 1,05 \\
\hline 8.35 & 0,88 & 1,12 & 1,06 & 1,10 & 1,08 \\
\hline 8.36 & 0,63 & 0,85 & 1,06 & 1,19 & 1,10 \\
\hline
\end{tabular}

Tabela 8.39: Expression Template / Klein - Soma de matrizes $(10 \leq n \leq 90)$ 
Quando rodamos para grande quantidade de elementos a Klein supera e muito essa técnica. Nesse momento identificamos que a utilização do laço varrendo quatro elementos de cada vez fez enorme diferença no tempo final, visto que a Klein utiliza a mesma técnica.

\begin{tabular}{|c|c|c|c|c|c|}
\hline$\frac{5}{\text { Tempo Expression }}$ & \multicolumn{5}{|c|}{ Número de elementos } \\
\hline Tempo Klein & $\mathbf{1 0 0}$ & $\mathbf{3 0 0}$ & $\mathbf{5 0 0}$ & $\mathbf{7 0 0}$ & $\mathbf{9 0 0}$ \\
\hline Função & 0,83 & 0,77 & 0,43 & 0,40 & 0,41 \\
\hline $\mathbf{8 . 2 8}$ & 0,89 & 0,80 & 0,42 & 0,41 & 0,48 \\
\hline $\mathbf{8 . 2 9}$ & 0,93 & 0,85 & 0,46 & 0,48 & 0,59 \\
\hline $\mathbf{8 . 3 0}$ & 0,98 & 0,90 & 0,51 & 0,56 & 0,64 \\
\hline $\mathbf{8 . 3 1}$ & 0,99 & 0,94 & 0,55 & 0,64 & 0,67 \\
\hline $\mathbf{8 . 3 2}$ & $\mathbf{1 , 0 1}$ & 0,98 & 0,59 & 0,71 & 0,68 \\
\hline $\mathbf{8 . 3 3}$ & $\mathbf{1 , 0 4}$ & $\mathbf{1 , 0 2}$ & 0,63 & 0,75 & 0,69 \\
\hline $\mathbf{8 . 3 4}$ & $\mathbf{1 , 1 5}$ & $\mathbf{1 , 0 6}$ & 0,65 & 0,75 & 0,71 \\
\hline $\mathbf{8 . 3 5}$ & $\mathbf{1 , 1 5}$ & $\mathbf{1 , 0 9}$ & 0,67 & 0,74 & 0,70 \\
\hline $\mathbf{8 . 3 6}$ & & & & & \\
\hline
\end{tabular}

Tabela 8.40: Expression Template / Klein - Soma de matrizes $(100 \leq n \leq 900)$

\section{Dummy / Klein}

A soma de matrizes utilizando a estratégia dummy é melhor quando somamos até três vetores. Porém ao aumentarmos a quantidade de elementos dos vetores a Klein aumenta sua vantagem até certo ponto e depois volta a descer.

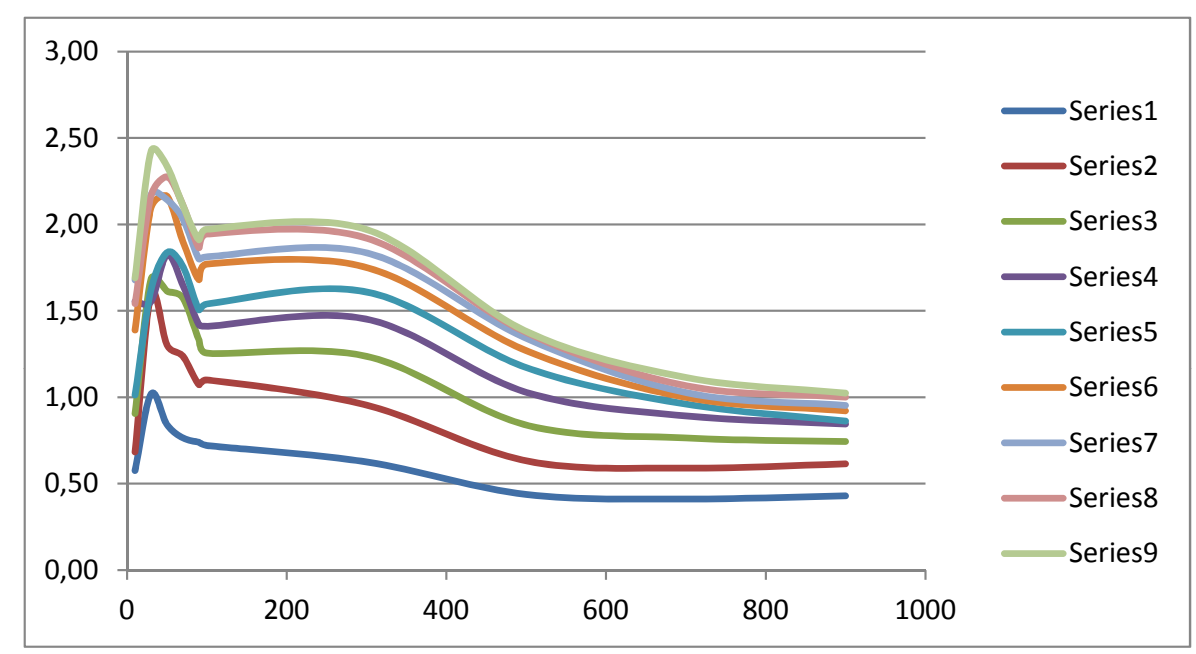

Figura 8.15: Soma de matrizes - Dummy / Klein 


\begin{tabular}{|c|c|c|c|c|c|}
\hline $\begin{array}{c}\text { Tempo Dummy } \\
\text { Tempo Klein }\end{array}$ & \multicolumn{5}{|c|}{ Número de elementos } \\
\hline Função & $\mathbf{1 0}$ & $\mathbf{3 0}$ & $\mathbf{5 0}$ & $\mathbf{7 0}$ & $\mathbf{9 0}$ \\
\hline $\mathbf{8 . 2 8}$ & 0,39 & 0,72 & 0,48 & 0,53 & 0,53 \\
\hline $\mathbf{8 . 2 9}$ & 0,72 & 0,88 & 0,87 & 0,95 & 0,84 \\
\hline $\mathbf{8 . 3 0}$ & 0,86 & $\mathbf{1 , 0 9}$ & $\mathbf{1 , 1 0}$ & $\mathbf{1 , 2 4}$ & $\mathbf{1 , 0 5}$ \\
\hline $\mathbf{8 . 3 1}$ & $\mathbf{1 , 2 0}$ & $\mathbf{1 , 1 6}$ & $\mathbf{1 , 1 2}$ & $\mathbf{1 , 2 8}$ & $\mathbf{1 , 1 1}$ \\
\hline $\mathbf{8 . 3 2}$ & $\mathbf{1 , 1 6}$ & $\mathbf{1 , 2 3}$ & $\mathbf{1 , 2 8}$ & $\mathbf{1 , 3 2}$ & $\mathbf{1 , 1 5}$ \\
\hline $\mathbf{8 . 3 3}$ & $\mathbf{1 , 2 4}$ & $\mathbf{1 , 2 6}$ & $\mathbf{1 , 3 5}$ & $\mathbf{1 , 3 4}$ & $\mathbf{1 , 1 7}$ \\
\hline $\mathbf{8 . 3 4}$ & $\mathbf{1 , 5 0}$ & $\mathbf{1 , 2 9}$ & $\mathbf{1 , 2 8}$ & $\mathbf{1 , 4 8}$ & $\mathbf{1 , 2 7}$ \\
\hline $\mathbf{8 . 3 5}$ & $\mathbf{1 , 3 5}$ & $\mathbf{1 , 2 3}$ & $\mathbf{1 , 3 5}$ & $\mathbf{1 , 4 7}$ & $\mathbf{1 , 4 3}$ \\
\hline $\mathbf{8 . 3 6}$ & $\mathbf{1 , 4 7}$ & $\mathbf{1 , 3 4}$ & $\mathbf{1 , 3 7}$ & $\mathbf{1 , 4 6}$ & $\mathbf{1 , 3 5}$ \\
\hline
\end{tabular}

Tabela 8.41: Dummy / Klein - Soma de matrizes $(10 \leq n \leq 90)$

A partir de $700 \times 700$ elementos a Klein perde em todos os casos para a estratégia dummy.

\begin{tabular}{|c|c|c|c|c|c|}
\hline$\frac{\text { Tempo Dummy }}{\text { Tempo Klein }}$ & \multicolumn{5}{|c|}{ Número de elementos } \\
\hline Função & 100 & 300 & 500 & 700 & 900 \\
\hline 8.28 & 0,53 & 0,46 & 0,41 & 0,51 & 0,48 \\
\hline 8.29 & 0,85 & 0,74 & 0,60 & 0,65 & 0,57 \\
\hline 8.30 & 0,97 & 0,97 & 0,85 & 0,74 & 0,66 \\
\hline 8.31 & 1,08 & 1,14 & 0,95 & 0,81 & 0,73 \\
\hline 8.32 & $\overline{1,17}$ & 1,26 & 1,03 & 0,83 & 0,78 \\
\hline 8.33 & 1,24 & 1,39 & 1,10 & 0,82 & 0,82 \\
\hline 8.34 & 1,41 & 1,48 & 1,13 & 0,82 & 0,86 \\
\hline 8.35 & 1,37 & 1,57 & 1,13 & 0,84 & 0,95 \\
\hline 8.36 & 1,37 & 1,67 & 1,10 & 0,86 & 0,95 \\
\hline
\end{tabular}

Tabela 8.42: Dummy / Klein - Soma de matrizes (100 $\leq n \leq 900)$

\subsection{Soma de matriz vezes escalar}

Efetuamos testes de soma de matrizes pré-multiplicando por escalar.

$$
\begin{gathered}
r e s p=k_{1} * a+k_{2} * b, \\
r e s p=k_{1} * a+k_{2} * b+k_{3} * c, \\
r e s p=k_{1} * a+k_{2} * b+k_{3} * c+k_{4} * d, \\
r e s p=k_{1} * a+k_{2} * b+k_{3} * c+k_{4} * d+k_{5} * e, \\
r e s p=k_{1} * a+k_{2} * b+k_{3} * c+k_{4} * d+k_{5} * e+k_{6} * f, \\
r e s p=k_{1} * a+k_{2} * b+k_{3} * c+k_{4} * d+k_{5} * e+k_{6} * f+k_{7} * g, \\
r e s p=k_{1} * a+k_{2} * b+k_{3} * c+k_{4} * d+k_{5} * e+k_{6} * f+k_{7} * g+k_{8} * h, \\
r e s p=k_{1} * a+k_{2} * b+k_{3} * c+k_{4} * d+k_{5} * e+k_{6} * f+k_{7} * g+k_{8} * h+k_{9} * i,
\end{gathered}
$$

onde resp, $a, b, c, d, e, f, g, h, i$ e $j$ são matrizes, e $k_{1}, k_{2}, k_{3}, k_{4}, k_{5}, k_{6}, k_{7}, k_{8}, k_{9}$ e $k_{10}$ são escalares.

O código da biblioteca MKL foi escrito dessa forma, 


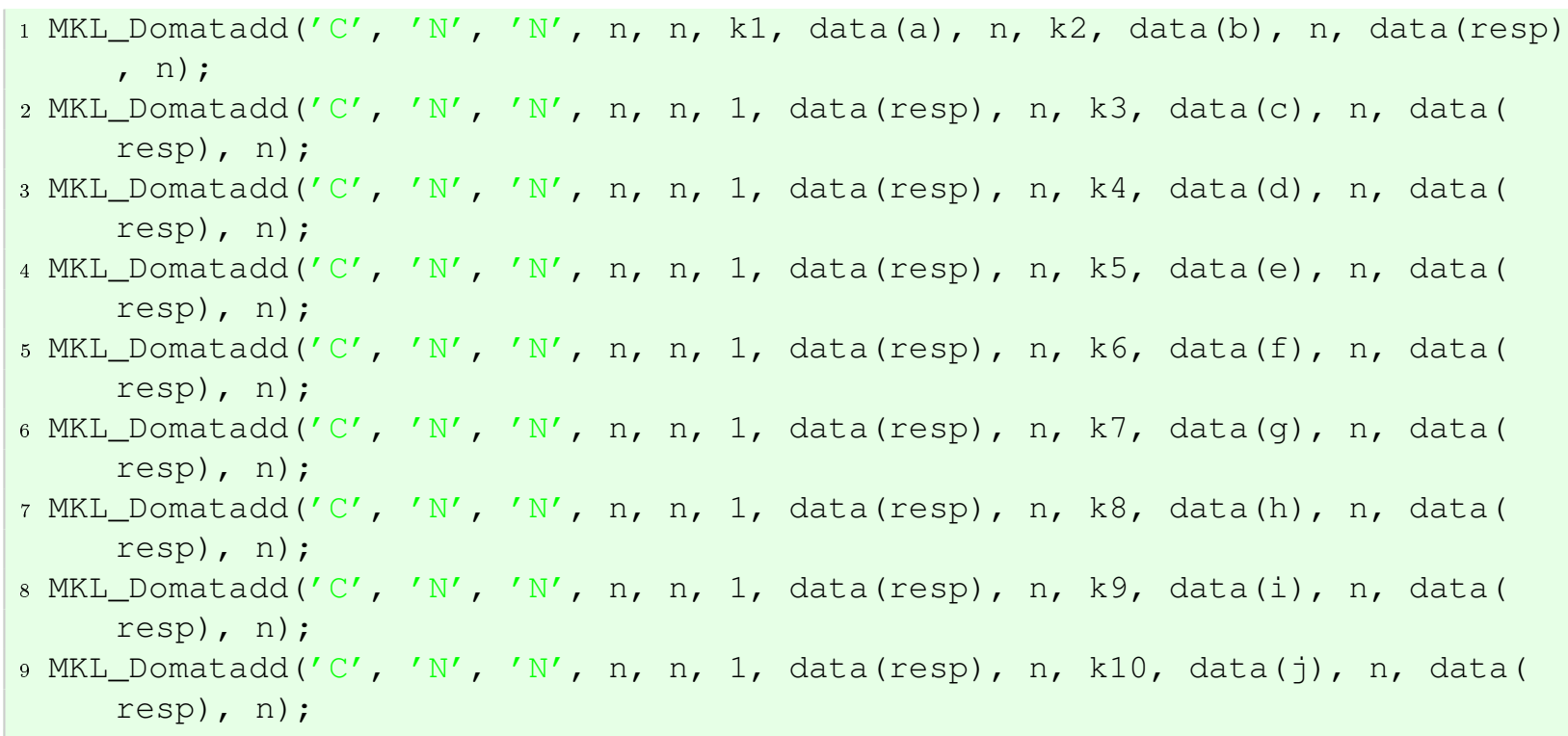

A ténica expression template foi implementada com dois laços:

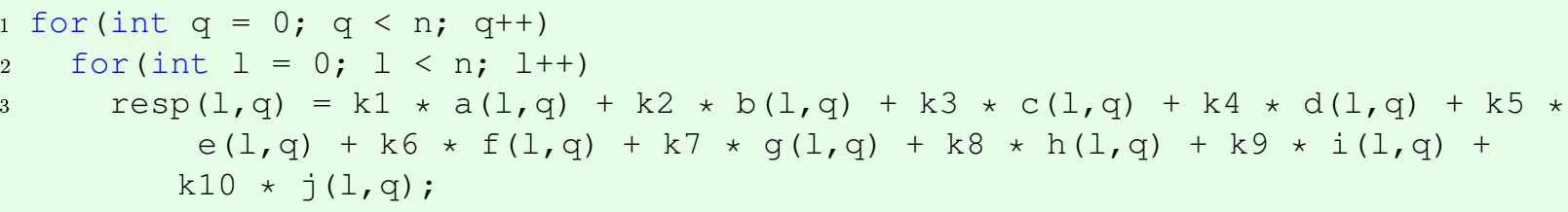

O código dummy fica dessa maneira:

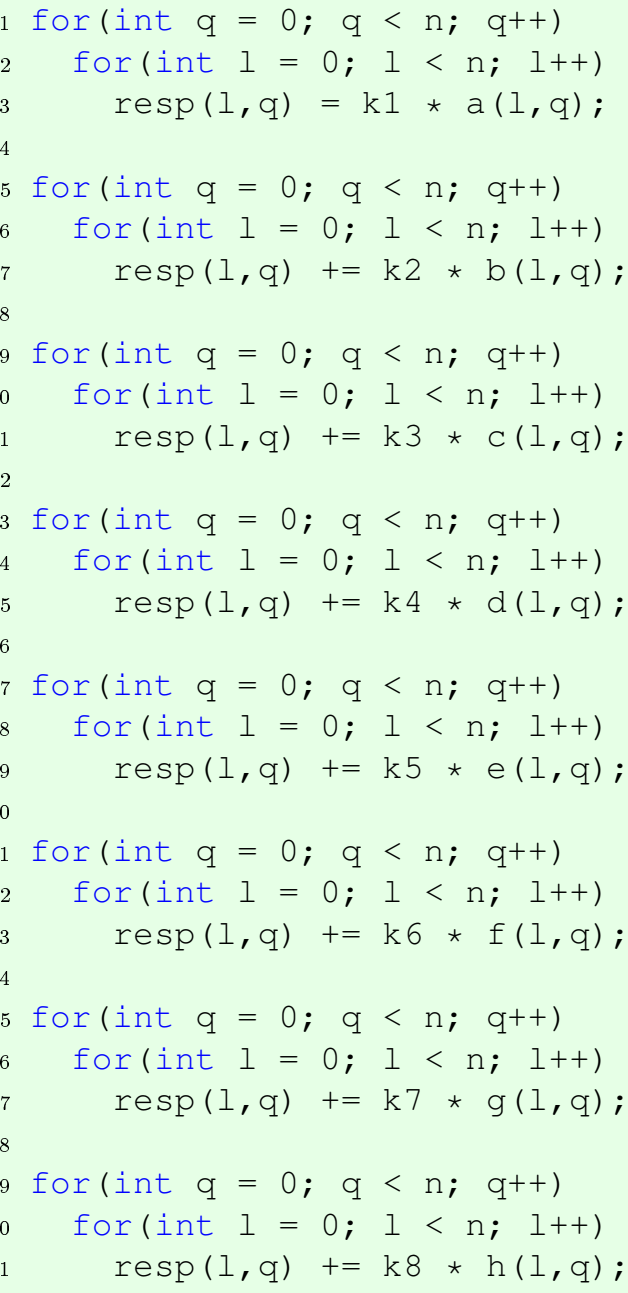




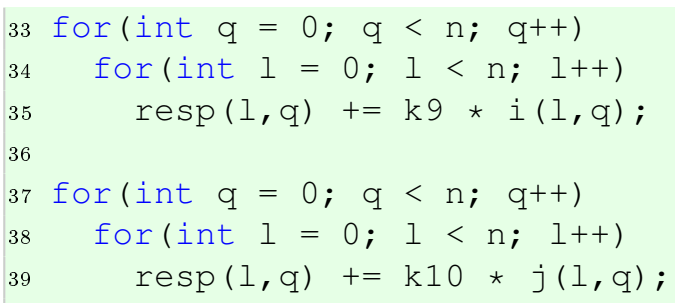

E o código da Klein, dessa maneira:

1 resp $=k 1 \star a+k 2 \star b+k 3 \star c+k 4 \star d+k 5 * e+k 6 \star f+k 7 \star g+k 8 \star h$ $+\mathrm{k} 9 * i+\mathrm{k} 10 * j$;

\section{MKL / Klein}

Nesse teste a Klein supera a MKL em alguns casos para vetores pequenos e outros para vetores maiores.

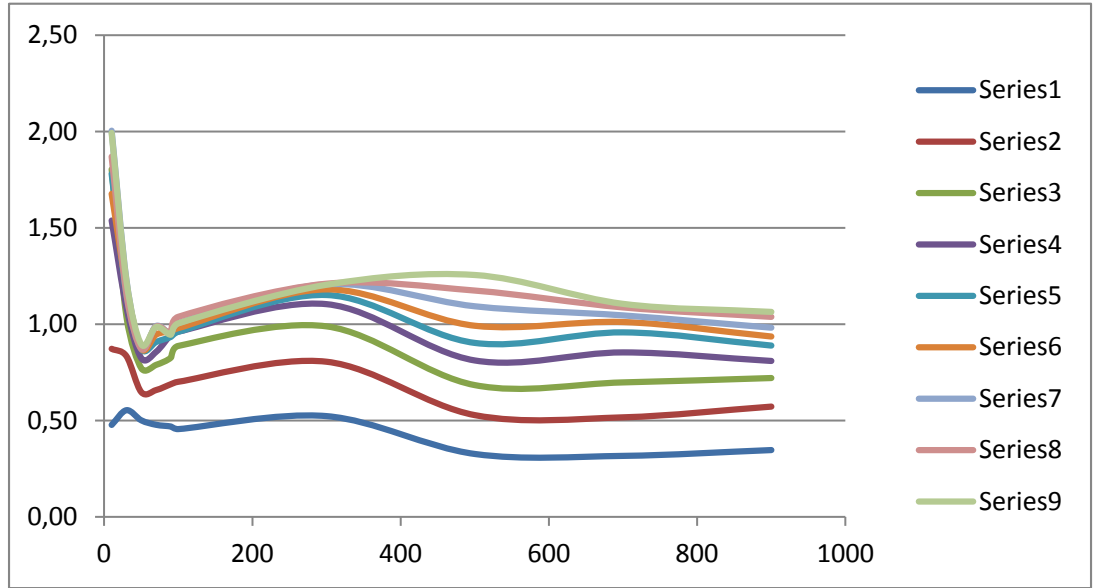

Figura 8.16: Multiplica escalar e soma matrizes - MKL / Klein

Quando operamos matrizes de dimensões 10x10 e 30x30 a Klein teve bom desempenho, ganhando em vários casos.

\begin{tabular}{|c|c|c|c|c|c|}
\hline $\begin{array}{c}\text { Tempo MKL } \\
\text { Tempo Klein }\end{array}$ & \multicolumn{5}{|c|}{ Número de elementos } \\
\hline Função & $\mathbf{1 0}$ & $\mathbf{3 0}$ & $\mathbf{5 0}$ & $\mathbf{7 0}$ & $\mathbf{9 0}$ \\
\hline $\mathbf{8 . 3 7}$ & 0,48 & 0,44 & 0,42 & 0,39 & 0,40 \\
\hline $\mathbf{8 . 3 8}$ & 0,84 & 0,79 & 0,64 & 0,64 & 0,66 \\
\hline $\mathbf{8 . 3 9}$ & $\mathbf{1 , 8 6}$ & $\mathbf{1 , 0 1}$ & 0,77 & 0,77 & 0,81 \\
\hline $\mathbf{8 . 4 0}$ & $\mathbf{1 , 6 1}$ & $\mathbf{1 , 1 1}$ & 0,79 & 0,88 & 0,94 \\
\hline $\mathbf{8 . 4 1}$ & $\mathbf{1 , 3 6}$ & $\mathbf{1 , 1 5}$ & 0,88 & 0,92 & 0,95 \\
\hline $\mathbf{8 . 4 2}$ & $\mathbf{1 , 7 5}$ & $\mathbf{1 , 1 9}$ & 0,89 & 0,96 & 0,97 \\
\hline $\mathbf{8 . 4 3}$ & $\mathbf{2 , 0 9}$ & $\mathbf{1 , 1 1}$ & 0,89 & $\mathbf{1 , 0 0}$ & 0,96 \\
\hline $\mathbf{8 . 4 4}$ & $\mathbf{1 , 9 5}$ & $\mathbf{1 , 1 8}$ & 0,89 & $\mathbf{1 , 0 0}$ & 0,96 \\
\hline $\mathbf{8 . 4 5}$ & $\mathbf{1 , 8 2}$ & $\mathbf{1 , 2 6}$ & 0,91 & $\mathbf{1 , 0 0}$ & 0,96 \\
\hline
\end{tabular}

Tabela 8.43: MKL / Klein - Soma de matriz vezes escalar $(10 \leq n \leq 90)$

Ao aumentar o número de elementos das matrizes notamos que mesmo com um grande número 
de elementos a Klein continua sendo competitiva nos casos em que operamos sobre mais de três matrizes.

\begin{tabular}{|c|c|c|c|c|c|}
\hline $\begin{array}{c}\text { Tempo MKL } \\
\text { Tempo Klein }\end{array}$ & \multicolumn{5}{|c|}{ Número de elementos } \\
\hline Função & $\mathbf{1 0 0}$ & $\mathbf{3 0 0}$ & $\mathbf{5 0 0}$ & $\mathbf{7 0 0}$ & $\mathbf{9 0 0}$ \\
\hline $\mathbf{8 . 3 7}$ & 0,40 & 0,50 & 0,32 & 0,31 & 0,34 \\
\hline $\mathbf{8 . 3 8}$ & 0,69 & 0,79 & 0,52 & 0,52 & 0,57 \\
\hline $\mathbf{8 . 3 9}$ & 0,87 & 0,98 & 0,68 & 0,70 & 0,73 \\
\hline $\mathbf{8 . 4 0}$ & 0,97 & $\mathbf{1 , 1 1}$ & 0,81 & 0,85 & 0,81 \\
\hline $\mathbf{8 . 4 1}$ & 0,99 & $\mathbf{1 , 1 5}$ & 0,90 & 0,96 & 0,89 \\
\hline $\mathbf{8 . 4 2}$ & $\mathbf{1 , 0 0}$ & $\mathbf{1 , 1 8}$ & 0,99 & $\mathbf{1 , 0 1}$ & 0,94 \\
\hline $\mathbf{8 . 4 3}$ & $\mathbf{1 , 0 1}$ & $\mathbf{1 , 2 1}$ & $\mathbf{1 , 0 9}$ & $\mathbf{1 , 0 5}$ & 0,98 \\
\hline $\mathbf{8 . 4 4}$ & $\mathbf{1 , 0 5}$ & $\mathbf{1 , 2 1}$ & $\mathbf{1 , 1 8}$ & $\mathbf{1 , 0 8}$ & $\mathbf{1 , 0 4}$ \\
\hline $\mathbf{8 . 4 5}$ & $\mathbf{1 , 0 1}$ & $\mathbf{1 , 1 9}$ & $\mathbf{1 , 2 6}$ & $\mathbf{1 , 1 0}$ & $\mathbf{1 , 0 6}$ \\
\hline
\end{tabular}

Tabela 8.44: $M K L /$ Klein - Soma de matriz vezes escalar $(100 \leq n \leq 900)$

\section{Expression template $\mathrm{x}$ Klein}

A técnica expression template ganha para vetores bastante pequenos e vetores muito grandes.

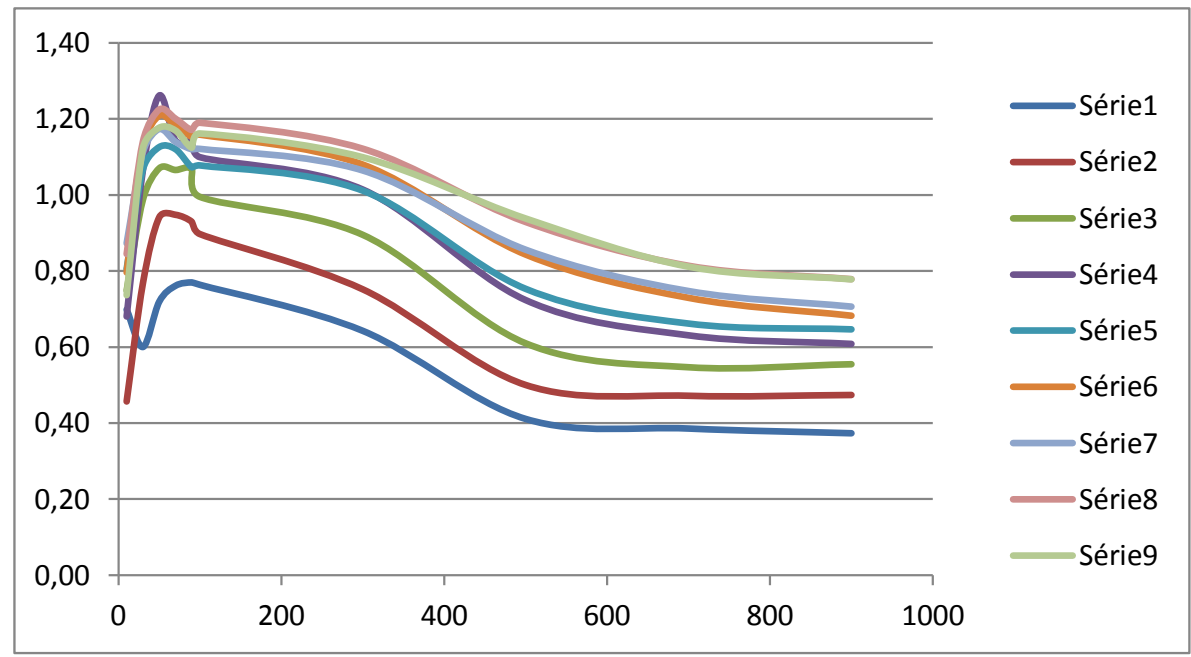

Figura 8.17: Multiplica escalar e soma matrizes - Expression / Klein 


\begin{tabular}{|c|c|c|c|c|c|}
\hline $\begin{array}{c}\text { Tempo Expression } \\
\text { Tempo Klein }\end{array}$ & \multicolumn{4}{|c|}{ Número de elementos } \\
\hline Função & $\mathbf{1 0}$ & $\mathbf{3 0}$ & $\mathbf{5 0}$ & $\mathbf{7 0}$ & $\mathbf{9 0}$ \\
\hline $\mathbf{8 . 3 7}$ & 0,37 & 0,71 & 0,84 & 0,77 & 0,78 \\
\hline $\mathbf{8 . 3 8}$ & 0,46 & 0,97 & 0,95 & 0,95 & 0,92 \\
\hline $\mathbf{8 . 3 9}$ & 0,75 & $\mathbf{1 , 0 8}$ & $\mathbf{1 , 0 6}$ & $\mathbf{1 , 0 5}$ & $\mathbf{1 , 0 3}$ \\
\hline $\mathbf{8 . 4 0}$ & 0,71 & $\mathbf{1 , 0 8}$ & $\mathbf{1 , 0 9}$ & $\mathbf{1 , 1 4}$ & $\mathbf{1 , 1 2}$ \\
\hline $\mathbf{8 . 4 1}$ & 0,61 & $\mathbf{1 , 1 0}$ & $\mathbf{1 , 1 8}$ & $\mathbf{1 , 1 5}$ & $\mathbf{1 , 1 3}$ \\
\hline $\mathbf{8 . 4 2}$ & 0,80 & $\mathbf{1 , 1 4}$ & $\mathbf{1 , 1 9}$ & $\mathbf{1 , 1 7}$ & $\mathbf{1 , 1 5}$ \\
\hline $\mathbf{8 . 4 3}$ & 0,93 & $\mathbf{1 , 0 6}$ & $\mathbf{1 , 2 2}$ & $\mathbf{1 , 2 0}$ & $\mathbf{1 , 1 7}$ \\
\hline $\mathbf{8 . 4 4}$ & 0,85 & $\mathbf{1 , 1 3}$ & $\mathbf{1 , 2 2}$ & $\mathbf{1 , 1 9}$ & $\mathbf{1 , 1 7}$ \\
\hline $\mathbf{8 . 4 5}$ & 0,76 & $\mathbf{1 , 1 6}$ & $\mathbf{1 , 1 9}$ & $\mathbf{1 , 1 9}$ & $\mathbf{1 , 1 7}$ \\
\hline
\end{tabular}

Tabela 8.45: Expression Template / Klein - Soma de matriz vezes escalar $(10 \leq n \leq 90)$

E essa diferença aumenta ao utilizarmos matrizes cada vez maiores com dimensões até 900x900.

\begin{tabular}{|c|c|c|c|c|c|}
\hline$\frac{\text { Tempo Expression }}{\text { Tempo Klein }}$ & \multicolumn{5}{|c|}{ Número de elementos } \\
\hline Função & 100 & 300 & 500 & 700 & 900 \\
\hline 8.37 & 0,76 & 0,64 & 0,41 & 0,39 & 0,37 \\
\hline 8.38 & 0,91 & 0,75 & 0,50 & 0,47 & 0,48 \\
\hline 8.39 & 0,99 & 0,89 & 0,61 & 0,54 & 0,56 \\
\hline 8.40 & 1,10 & 1,02 & 0,73 & 0,63 & 0,61 \\
\hline 8.41 & 1,14 & 1,06 & 0,79 & 0,70 & 0,66 \\
\hline 8.42 & 1,17 & 1,08 & 0,85 & 0,73 & 0,68 \\
\hline 8.43 & 1,21 & 1,11 & 0,91 & 0,78 & 0,74 \\
\hline 8.44 & 1,20 & 1,12 & 0,93 & 0,82 & 0,78 \\
\hline 8.45 & 1,20 & 1,11 & 0,95 & 0,82 & 0,79 \\
\hline
\end{tabular}

Tabela 8.46: Expression Template / Klein - Soma de matriz vezes escalar $(100 \leq n \leq 900)$

\section{Dummy / Klein}

Para o caso de soma de escalar vezes matriz o comportamento é parecido com a soma de matrizes. A Klein aumenta sua vantagem ao aumentarmos a quantidade de elementos das matrizes. 


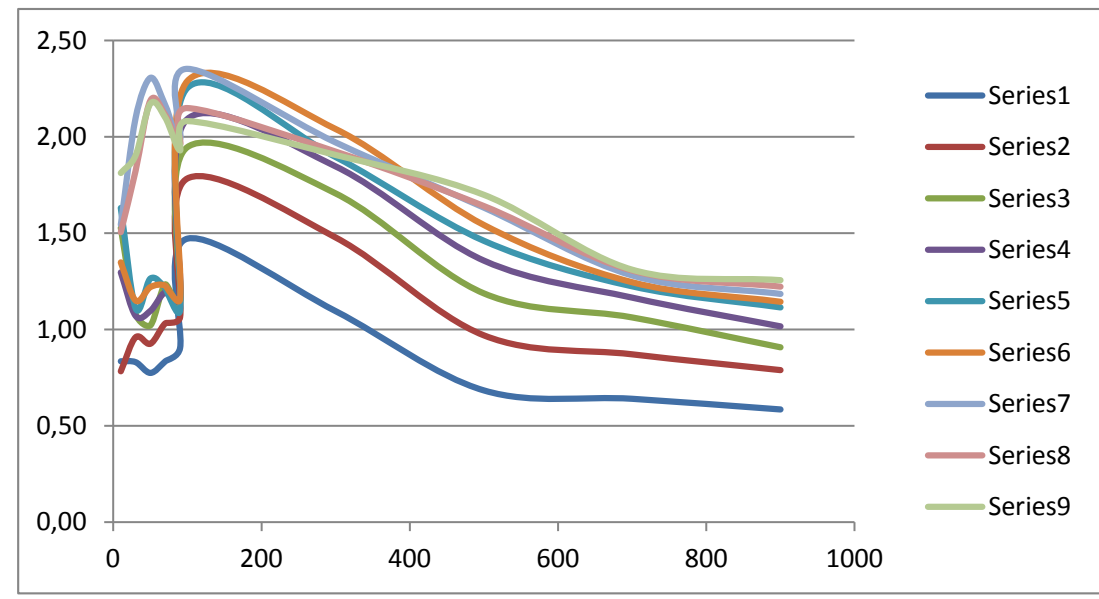

Figura 8.18: Multiplica escalar e soma matrizes - Dummy / Klein

\begin{tabular}{|c|c|c|c|c|c|}
\hline $\begin{array}{c}\text { Tempo Dummy } \\
\text { Tempo Klein }\end{array}$ & \multicolumn{5}{|c|}{ Número de elementos } \\
\hline Função & $\mathbf{1 0}$ & $\mathbf{3 0}$ & $\mathbf{5 0}$ & $\mathbf{7 0}$ & $\mathbf{9 0}$ \\
\hline $\mathbf{8 . 3 7}$ & 0,84 & 0,83 & 0,78 & 0,84 & 0,90 \\
\hline $\mathbf{8 . 3 8}$ & 0,78 & 0,96 & 0,93 & $\mathbf{1 , 0 3}$ & $\mathbf{1 , 0 6}$ \\
\hline $\mathbf{8 . 3 9}$ & $\mathbf{1 , 5 3}$ & $\mathbf{1 , 0 8}$ & $\mathbf{1 , 0 2}$ & $\mathbf{1 , 2 3}$ & $\mathbf{1 , 1 4}$ \\
\hline $\mathbf{8 . 4 0}$ & $\mathbf{1 , 3 0}$ & $\mathbf{1 , 0 7}$ & $\mathbf{1 , 1 0}$ & $\mathbf{1 , 1 9}$ & $\mathbf{1 , 1 6}$ \\
\hline $\mathbf{8 . 4 1}$ & $\mathbf{1 , 6 3}$ & $\mathbf{1 , 1 1}$ & $\mathbf{1 , 2 6}$ & $\mathbf{1 , 2 1}$ & $\mathbf{1 , 1 0}$ \\
\hline $\mathbf{8 . 4 2}$ & $\mathbf{1 , 3 5}$ & $\mathbf{1 , 1 5}$ & $\mathbf{1 , 2 2}$ & $\mathbf{1 , 2 3}$ & $\mathbf{1 , 1 6}$ \\
\hline $\mathbf{8 . 4 3}$ & $\mathbf{1 , 3 8}$ & $\mathbf{1 , 1 0}$ & $\mathbf{1 , 1 5}$ & $\mathbf{1 , 2 8}$ & $\mathbf{1 , 2 0}$ \\
\hline $\mathbf{8 . 4 4}$ & $\mathbf{1 , 2 1}$ & $\mathbf{1 , 0 8}$ & $\mathbf{1 , 1 2}$ & $\mathbf{1 , 2 8}$ & $\mathbf{1 , 1 5}$ \\
\hline $\mathbf{8 . 4 5}$ & $\mathbf{1 , 2 5}$ & $\mathbf{1 , 0 8}$ & $\mathbf{1 , 1 4}$ & $\mathbf{1 , 2 3}$ & $\mathbf{1 , 1 1}$ \\
\hline
\end{tabular}

Tabela 8.47: Dummy / Klein - Soma de matriz vezes escalar $(10 \leq n \leq 90)$

Perde em todos os casos quando as matrizes chegam a 700 elementos, mas recupera um pouco em 900 elementos.

\begin{tabular}{|c|c|c|c|c|c|}
\hline $\begin{array}{c}\text { Tempo Dummy } \\
\text { Tempo Klein }\end{array}$ & \multicolumn{5}{|c|}{ Número de elementos } \\
\hline Função & $\mathbf{1 0 0}$ & $\mathbf{3 0 0}$ & $\mathbf{5 0 0}$ & $\mathbf{7 0 0}$ & $\mathbf{9 0 0}$ \\
\hline $\mathbf{8 . 3 7}$ & 0,87 & 0,71 & 0,50 & 0,56 & 0,55 \\
\hline $\mathbf{8 . 3 8}$ & $\mathbf{1 , 0 0}$ & 0,98 & 0,71 & 0,72 & 0,69 \\
\hline $\mathbf{8 . 3 9}$ & $\mathbf{1 , 1 3}$ & $\mathbf{1 , 1 2}$ & 0,87 & 0,82 & 0,75 \\
\hline $\mathbf{8 . 4 0}$ & $\mathbf{1 , 1 5}$ & $\mathbf{1 , 2 5}$ & $\mathbf{1 , 0 0}$ & 0,89 & 0,82 \\
\hline $\mathbf{8 . 4 1}$ & $\mathbf{1 , 2 7}$ & $\mathbf{1 , 2 7}$ & $\mathbf{1 , 0 4}$ & 0,90 & 0,88 \\
\hline $\mathbf{8 . 4 2}$ & $\mathbf{1 , 2 1}$ & $\mathbf{1 , 2 7}$ & $\mathbf{1 , 0 8}$ & 0,89 & 0,92 \\
\hline $\mathbf{8 . 4 3}$ & $\mathbf{1 , 2 1}$ & $\mathbf{1 , 2 9}$ & $\mathbf{1 , 1 2}$ & 0,90 & 0,96 \\
\hline $\mathbf{8 . 4 4}$ & $\mathbf{1 , 2 0}$ & $\mathbf{1 , 3 0}$ & $\mathbf{1 , 1 3}$ & 0,93 & $\mathbf{1 , 0 0}$ \\
\hline $\mathbf{8 . 4 5}$ & $\mathbf{1 , 2 0}$ & $\mathbf{1 , 3 0}$ & $\mathbf{1 , 1 4}$ & 0,93 & $\mathbf{1 , 0 3}$ \\
\hline
\end{tabular}

Tabela 8.48: Dummy / Klein - Soma de matriz vezes escalar (100 $\leq n \leq 900)$ 


\subsection{Soma e subtração de matriz vezes escalar}

Para nos aproximarmos de operações reais, efetuamos os testes novamente para expressões de soma e subtração de matrizes.

$$
\begin{gathered}
r e s p=k_{1} * a-k_{2} * b, \\
r e s p=k_{1} * a-k_{2} * b+k_{3} * c, \\
r e s p=k_{1} * a+k_{2} * b-k_{3} * c+k_{4} * d, \\
r e s p=k_{1} * a-k_{2} * b+k_{3} * c+k_{4} * d-k_{5} * e, \\
r e s p=-k_{1} * a+k_{2} * b-k_{3} * c+k_{4} * d-k_{5} * e+k_{6} * f, \\
r e s p=k_{1} * a-k_{2} * b+k_{3} * c-k_{4} * d+k_{5} * e-k_{6} * f-k_{7} * g, \\
r e s p=-k_{1} * a+k_{2} * b-k_{3} * c-k_{4} * d+k_{5} * e-k_{6} * f+k_{7} * g-k_{8} * h, \\
r e s p=k_{1} * a-k_{2} * b-k_{3} * c+k_{4} * d+k_{5} * e-k_{6} * f-k_{7} * g+k_{8} * h+k_{9} * i, \\
r e s p=-k_{1} * a-k_{2} * b+k_{3} * c-k_{4} * d+k_{5} * e-k_{6} * f+k_{7} * g+k_{8} * h-k_{9} * i-k_{10} * j,
\end{gathered}
$$

onde resp, $a, b, c, d, e, f, g, h, i$ e $j$ são matrizes, e $k_{1}, k_{2}, k_{3}, k_{4}, k_{5}, k_{6}, k_{7}, k_{8}, k_{9}$ e $k_{10}$ são escalares.

O código da biblioteca MKL foi escrito dessa forma,

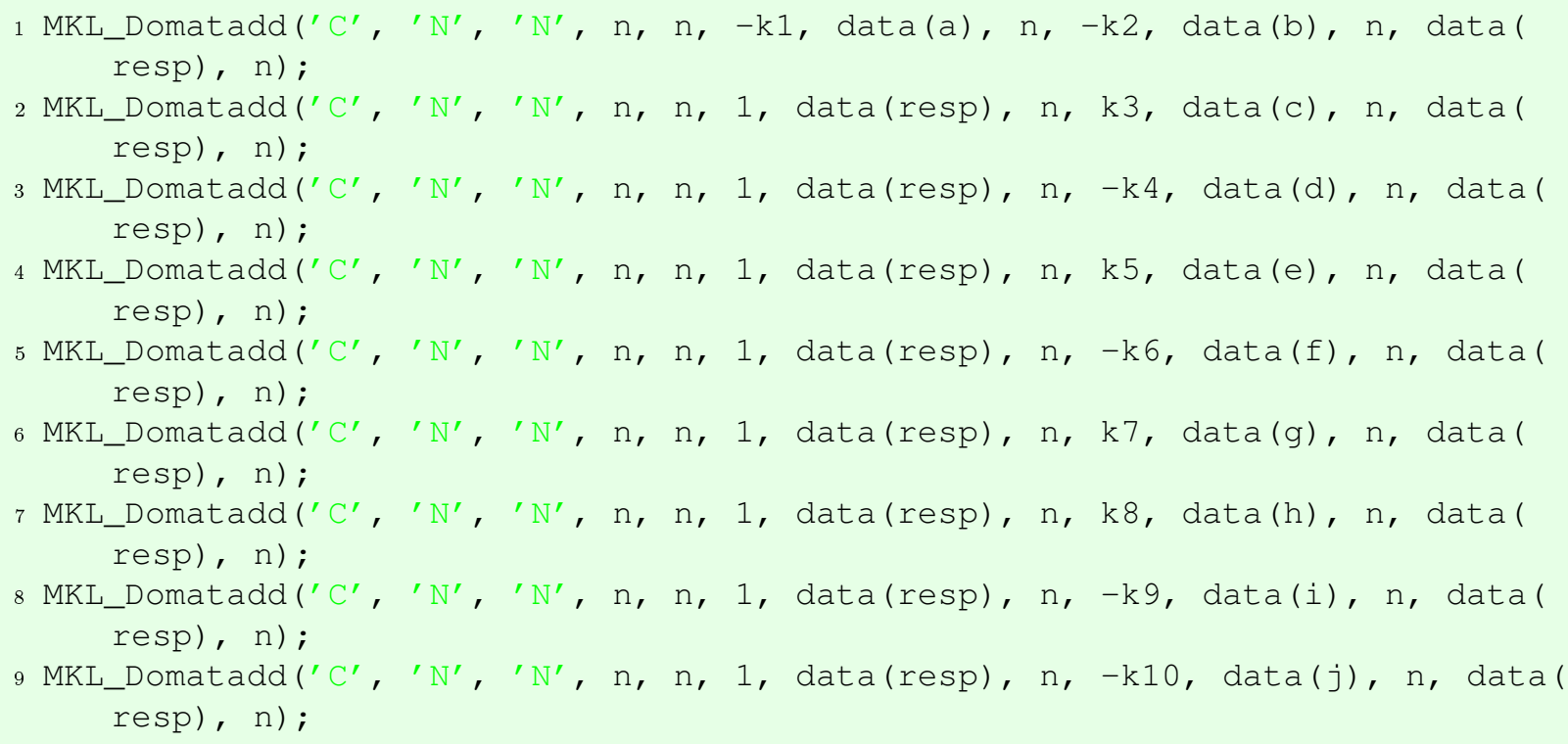

a ténica expression template foi implementada com dois laços:

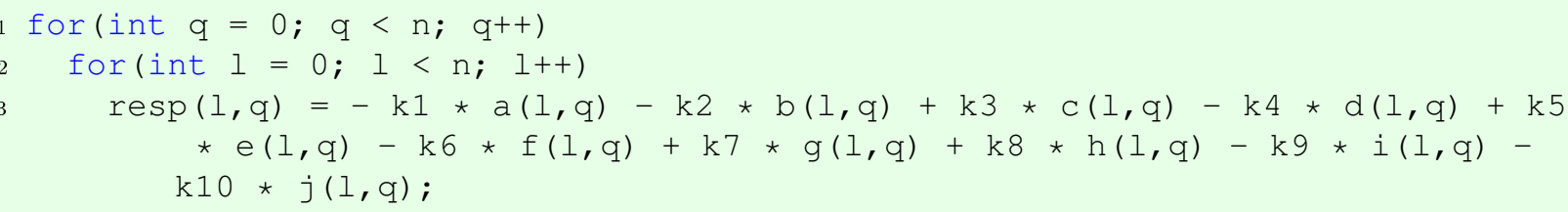

o código dummy fica dessa maneira:

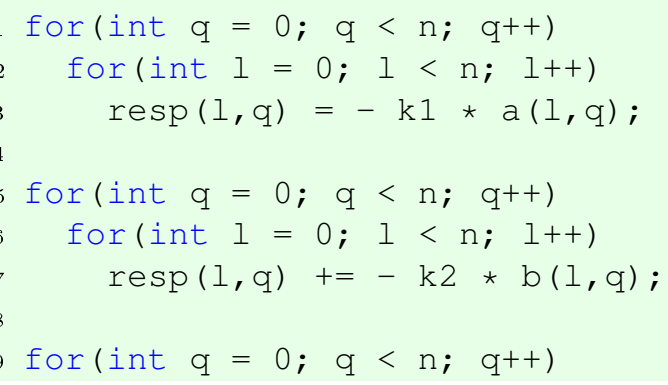




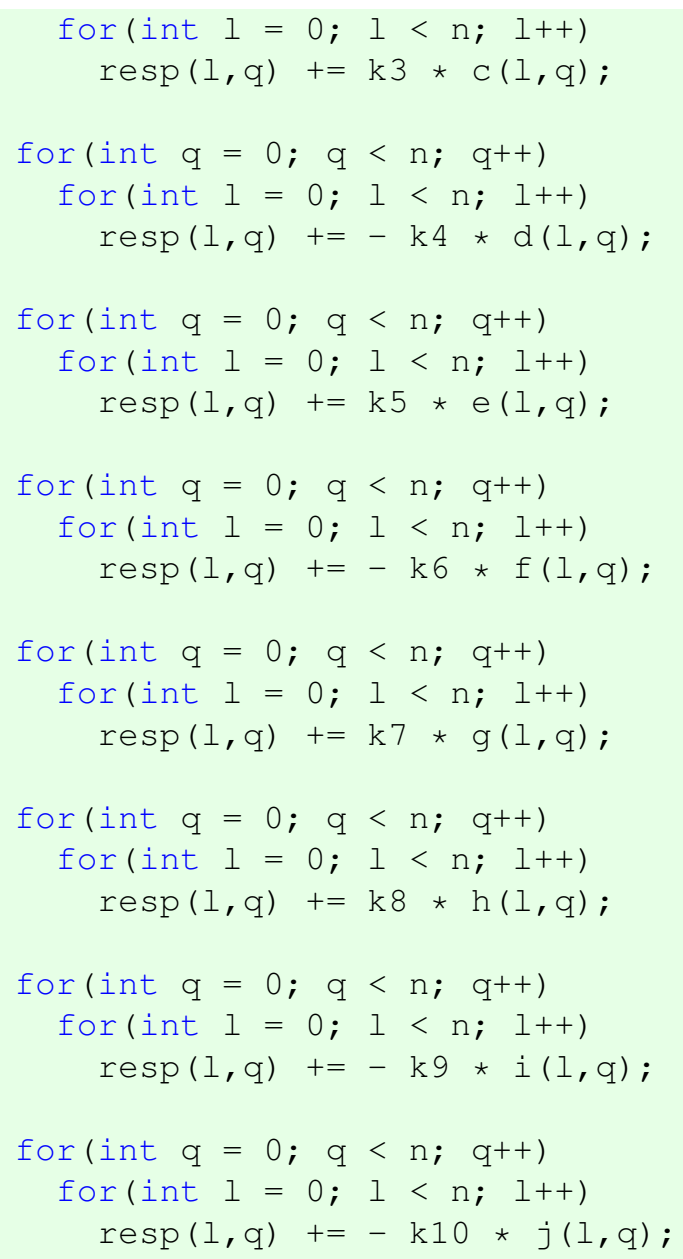

e a Klein dessa forma:

$1 \operatorname{resp}=-\mathrm{k} 1$ *a $-\mathrm{k} 2 \star \mathrm{b}+\mathrm{k} 3 \star \mathrm{c}-\mathrm{k} 4 \star \mathrm{d}+\mathrm{k} 5 \star \mathrm{e}-\mathrm{k} 6 \star \mathrm{f}+\mathrm{k} 7 \star \mathrm{g}+\mathrm{k} 8$ * $\mathrm{h}-\mathrm{k} 9 \star \mathrm{i}-\mathrm{k} 10 * \mathrm{j}$;

\section{MKL / Klein}

O desempenho da MKL com relação a Klein foi semelhante à soma de matrizes. A Klein ganha para vetores pequenos quando são efetuadas mais de três operações, porém sempre perde para poucas operações. 


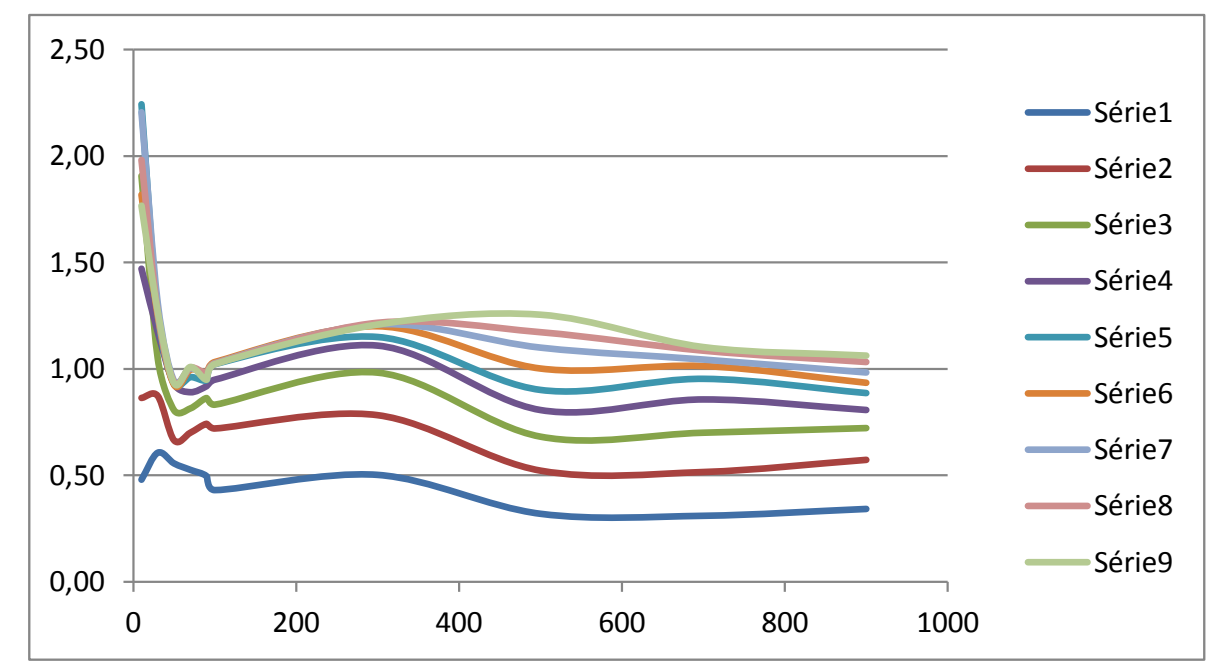

Figura 8.19: Multiplica escalar e soma matrizes - MKL / Klein

A Klein superou nos vetores menores com dimensões entre 10x10 e 30x30 e foi competitivo nos casos em que operamos sobre mais de 3 vetores.

\begin{tabular}{|c|c|c|c|c|c|}
\hline $\begin{array}{c}\text { Tempo MKL } \\
\text { Tempo Klein }\end{array}$ & \multicolumn{5}{|c|}{ Número de elementos } \\
\hline Função & $\mathbf{1 0}$ & $\mathbf{3 0}$ & $\mathbf{5 0}$ & $\mathbf{7 0}$ & $\mathbf{9 0}$ \\
\hline $\mathbf{8 . 4 6}$ & 0,48 & 0,42 & 0,43 & 0,43 & 0,42 \\
\hline $\mathbf{8 . 4 7}$ & 0,85 & 0,74 & 0,68 & 0,67 & 0,73 \\
\hline $\mathbf{8 . 4 8}$ & $\mathbf{1 , 9 1}$ & $\mathbf{1 , 0 5}$ & 0,81 & 0,81 & 0,86 \\
\hline $\mathbf{8 . 4 9}$ & $\mathbf{1 , 4 7}$ & $\mathbf{1 , 1 7}$ & 0,93 & 0,89 & 0,92 \\
\hline $\mathbf{8 . 5 0}$ & $\mathbf{2 , 2 4}$ & $\mathbf{1 , 2 5}$ & 0,93 & 0,96 & 0,95 \\
\hline $\mathbf{8 . 5 1}$ & $\mathbf{1 , 8 2}$ & $\mathbf{1 , 2 5}$ & 0,92 & $\mathbf{1 , 0 0}$ & 0,98 \\
\hline $\mathbf{8 . 5 2}$ & $\mathbf{2 , 2 1}$ & $\mathbf{1 , 3 1}$ & 0,94 & $\mathbf{1 , 0 0}$ & 0,97 \\
\hline $\mathbf{8 . 5 3}$ & $\mathbf{1 , 9 8}$ & $\mathbf{1 , 2 6}$ & 0,94 & $\mathbf{1 , 0 1}$ & 0,98 \\
\hline $\mathbf{8 . 5 4}$ & $\mathbf{1 , 7 7}$ & $\mathbf{1 , 2 7}$ & 0,93 & $\mathbf{1 , 0 1}$ & 0,95 \\
\hline
\end{tabular}

Tabela 8.49: $M K L /$ Klein - Soma e subtração de matriz vezes escalar $(10 \leq n \leq 90)$

Identificamos uma oscilação de comportamento para matrizes com dimensões entre 100x100 e 900x900. Ao analisarmos as matrizes com dimensões 100x100 e 300x300 acreditamos que a medida que aumentássemos o número de elementos a Klein passaria a superar a MKL em mais casos, porém não foi o que ocorreu. A partir da dimensão 500x500 a MKL volta a aumentar sua vantagem perante a Klein. 


\begin{tabular}{|c|c|c|c|c|c|}
\hline $\begin{array}{c}\text { Tempo MKL } \\
\text { Tempo Klein }\end{array}$ & \multicolumn{5}{|c|}{ Número de elementos } \\
\hline Função & $\mathbf{1 0 0}$ & $\mathbf{3 0 0}$ & $\mathbf{5 0 0}$ & $\mathbf{7 0 0}$ & $\mathbf{9 0 0}$ \\
\hline $\mathbf{8 . 4 6}$ & 0,43 & 0,50 & 0,32 & 0,31 & 0,34 \\
\hline $\mathbf{8 . 4 7}$ & 0,72 & 0,78 & 0,52 & 0,52 & 0,57 \\
\hline $\mathbf{8 . 4 8}$ & 0,83 & 0,98 & 0,68 & 0,70 & 0,72 \\
\hline $\mathbf{8 . 4 9}$ & 0,95 & $\mathbf{1 , 1 1}$ & 0,81 & 0,86 & 0,81 \\
\hline $\mathbf{8 . 5 0}$ & $\mathbf{1 , 0 2}$ & $\mathbf{1 , 1 5}$ & 0,90 & 0,95 & 0,89 \\
\hline $\mathbf{8 . 5 1}$ & $\mathbf{1 , 0 3}$ & $\mathbf{1 , 2 0}$ & $\mathbf{1 , 0 0}$ & $\mathbf{1 , 0 1}$ & 0,93 \\
\hline $\mathbf{8 . 5 2}$ & $\mathbf{1 , 0 3}$ & $\mathbf{1 , 2 1}$ & $\mathbf{1 , 1 0}$ & $\mathbf{1 , 0 4}$ & 0,98 \\
\hline $\mathbf{8 . 5 3}$ & $\mathbf{1 , 0 2}$ & $\mathbf{1 , 2 2}$ & $\mathbf{1 , 1 7}$ & $\mathbf{1 , 0 9}$ & $\mathbf{1 , 0 3}$ \\
\hline $\mathbf{8 . 5 4}$ & $\mathbf{1 , 0 2}$ & $\mathbf{1 , 2 1}$ & $\mathbf{1 , 2 5}$ & $\mathbf{1 , 1 0}$ & $\mathbf{1 , 0 6}$ \\
\hline
\end{tabular}

Tabela 8.50: $M K L /$ Klein - Soma e subtração de matriz vezes escalar $(100 \leq n \leq 900)$

\section{Expression template $\mathrm{x}$ Klein}

Para a técnica expression template a Klein perde nos extremos, quando as matrizes são pequenas ou grandes.

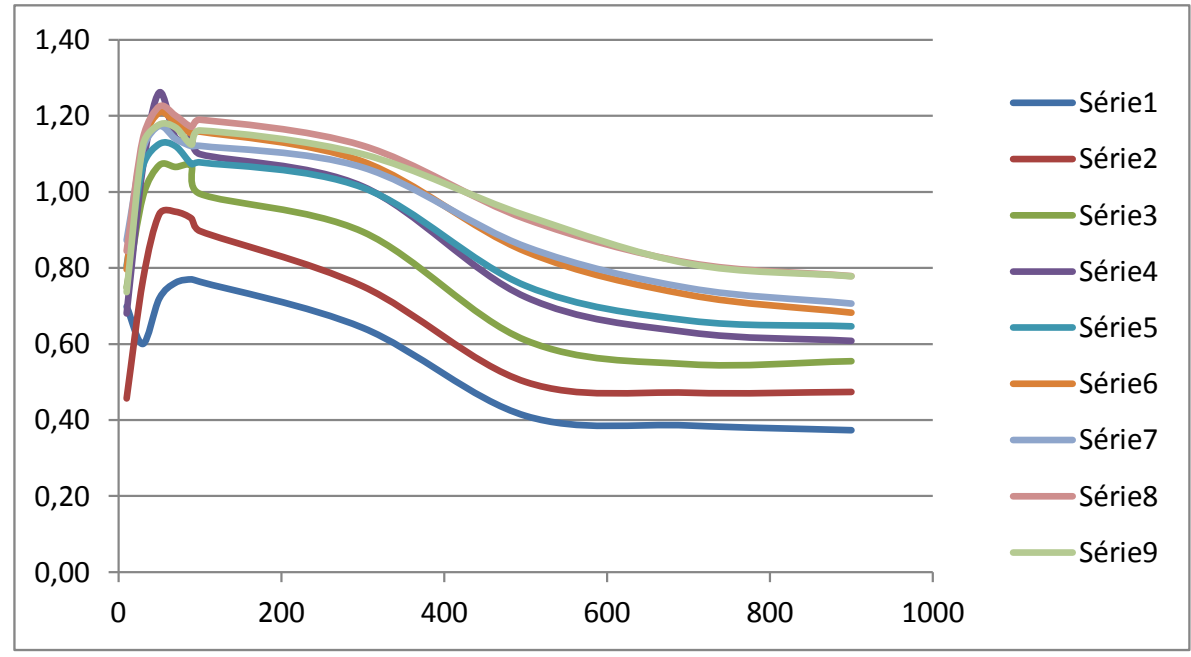

Figura 8.20: Multiplica escalar, soma e subtrai matrizes - Expression / Klein

\begin{tabular}{|c|c|c|c|c|c|}
\hline$\frac{\mid}{\text { Tempo Expression }}$ Tempo Klein & \multicolumn{5}{|c|}{ Número de elementos } \\
\hline Função & $\mathbf{1 0}$ & $\mathbf{3 0}$ & $\mathbf{5 0}$ & $\mathbf{7 0}$ & $\mathbf{9 0}$ \\
\hline $\mathbf{8 . 4 6}$ & 0,70 & 0,60 & 0,72 & 0,76 & 0,77 \\
\hline $\mathbf{8 . 4 7}$ & 0,46 & 0,77 & 0,94 & 0,95 & 0,93 \\
\hline $\mathbf{8 . 4 8}$ & 0,75 & 0,99 & $\mathbf{1 , 0 7}$ & $\mathbf{1 , 0 7}$ & $\mathbf{1 , 0 7}$ \\
\hline $\mathbf{8 . 4 9}$ & 0,68 & $\mathbf{1 , 0 7}$ & $\mathbf{1 , 2 6}$ & $\mathbf{1 , 1 4}$ & $\mathbf{1 , 1 4}$ \\
\hline $\mathbf{8 . 5 0}$ & 0,81 & $\mathbf{1 , 0 7}$ & $\mathbf{1 , 1 3}$ & $\mathbf{1 , 1 2}$ & $\mathbf{1 , 0 7}$ \\
\hline $\mathbf{8 . 5 1}$ & 0,80 & $\mathbf{1 , 1 3}$ & $\mathbf{1 , 2 1}$ & $\mathbf{1 , 1 8}$ & $\mathbf{1 , 1 5}$ \\
\hline $\mathbf{8 . 5 2}$ & 0,87 & $\mathbf{1 , 1 1}$ & $\mathbf{1 , 1 7}$ & $\mathbf{1 , 1 4}$ & $\mathbf{1 , 1 2}$ \\
\hline $\mathbf{8 . 5 3}$ & 0,84 & $\mathbf{1 , 1 4}$ & $\mathbf{1 , 2 2}$ & $\mathbf{1 , 2 0}$ & $\mathbf{1 , 1 7}$ \\
\hline $\mathbf{8 . 5 4}$ & 0,74 & $\mathbf{1 , 1 2}$ & $\mathbf{1 , 1 8}$ & $\mathbf{1 , 1 7}$ & $\mathbf{1 , 1 2}$ \\
\hline
\end{tabular}

Tabela 8.51: Expression Template / Klein - Soma e subtração de matriz vezes escalar $(10 \leq n \leq 90)$ 


\begin{tabular}{|c|c|c|c|c|c|}
\hline $\begin{array}{c}\text { Tempo Expression } \\
\text { Tempo Klein }\end{array}$ & \multicolumn{5}{|c|}{ Número de elementos } \\
\hline Função & $\mathbf{1 0 0}$ & $\mathbf{3 0 0}$ & $\mathbf{5 0 0}$ & $\mathbf{7 0 0}$ & $\mathbf{9 0 0}$ \\
\hline $\mathbf{8 . 4 6}$ & 0,76 & 0,64 & 0,41 & 0,39 & 0,37 \\
\hline $\mathbf{8 . 4 7}$ & 0,90 & 0,75 & 0,50 & 0,47 & 0,47 \\
\hline $\mathbf{8 . 4 8}$ & $\mathbf{1 , 0 0}$ & 0,90 & 0,61 & 0,55 & 0,55 \\
\hline $\mathbf{8 . 4 9}$ & $\mathbf{1 , 1 0}$ & $\mathbf{1 , 0 1}$ & 0,72 & 0,63 & 0,61 \\
\hline $\mathbf{8 . 5 0}$ & $\mathbf{1 , 0 8}$ & $\mathbf{1 , 0 1}$ & 0,75 & 0,66 & 0,65 \\
\hline $\mathbf{8 . 5 1}$ & $\mathbf{1 , 1 6}$ & $\mathbf{1 , 0 8}$ & 0,84 & 0,73 & 0,68 \\
\hline $\mathbf{8 . 5 2}$ & $\mathbf{1 , 1 2}$ & $\mathbf{1 , 0 6}$ & 0,86 & 0,75 & 0,71 \\
\hline $\mathbf{8 . 5 3}$ & $\mathbf{1 , 1 9}$ & $\mathbf{1 , 1 2}$ & 0,93 & 0,81 & 0,78 \\
\hline $\mathbf{8 . 5 4}$ & $\mathbf{1 , 1 6}$ & $\mathbf{1 , 1 0}$ & 0,94 & 0,81 & 0,78 \\
\hline
\end{tabular}

Tabela 8.52: Expression Template / Klein - Soma e subtração de matriz vezes escalar $(100 \leq n \leq 900)$

\section{Dummy / Klein}

Para soma e subtração de matriz vezes escalar o comportamento é praticamente o mesmo da soma de matriz vezes escalar. A Klein aumenta sua vantagem ao aumentarmos a quantidade de elementos das matrizes.

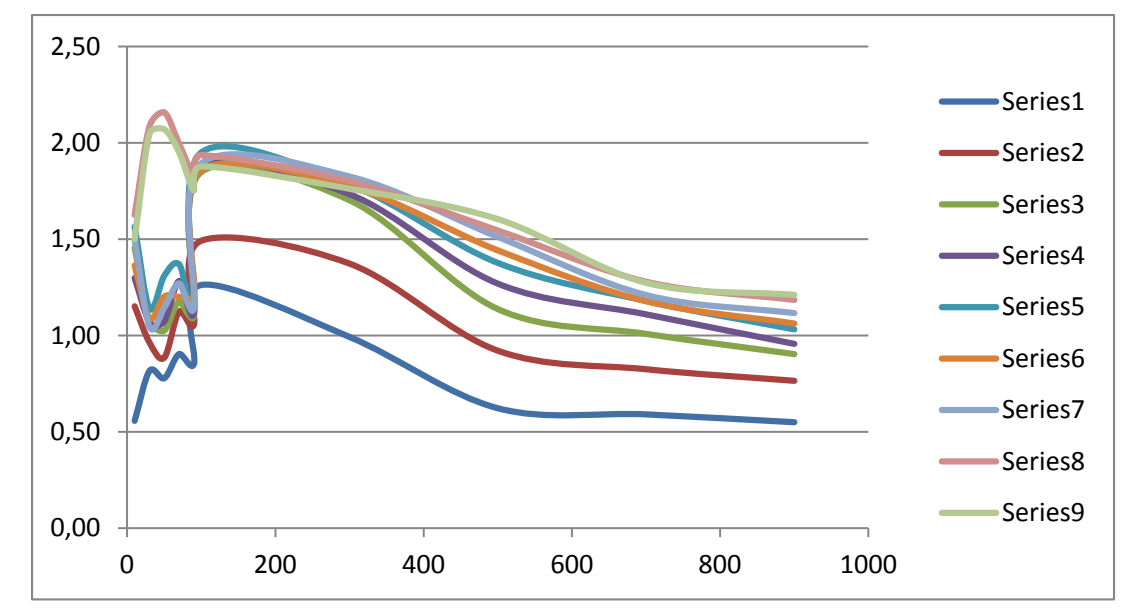

Figura 8.21: Multiplica escalar, soma e subtrai matrizes - Dummy / Klein 


\begin{tabular}{|c|c|c|c|c|c|}
\hline $\begin{array}{c}\text { Tempo Dummy } \\
\text { Tempo Klein }\end{array}$ & \multicolumn{5}{|c|}{ Número de elementos } \\
\hline Função & $\mathbf{1 0}$ & $\mathbf{3 0}$ & $\mathbf{5 0}$ & $\mathbf{7 0}$ & $\mathbf{9 0}$ \\
\hline $\mathbf{8 . 4 6}$ & 0,56 & 0,82 & 0,78 & 0,90 & 0,85 \\
\hline $\mathbf{8 . 4 7}$ & $\mathbf{1 , 1 5}$ & 0,96 & 0,89 & $\mathbf{1 , 1 2}$ & $\mathbf{1 , 0 6}$ \\
\hline $\mathbf{8 . 4 8}$ & $\mathbf{1 , 4 5}$ & $\mathbf{1 , 0 8}$ & $\mathbf{1 , 0 3}$ & $\mathbf{1 , 1 8}$ & $\mathbf{1 , 1 0}$ \\
\hline $\mathbf{8 . 4 9}$ & $\mathbf{1 , 3 0}$ & $\mathbf{1 , 0 7}$ & $\mathbf{1 , 0 7}$ & $\mathbf{1 , 2 8}$ & $\mathbf{1 , 1 2}$ \\
\hline $\mathbf{8 . 5 0}$ & $\mathbf{1 , 5 7}$ & $\mathbf{1 , 1 4}$ & $\mathbf{1 , 3 1}$ & $\mathbf{1 , 3 7}$ & $\mathbf{1 , 1 6}$ \\
\hline $\mathbf{8 . 5 1}$ & $\mathbf{1 , 3 6}$ & $\mathbf{1 , 0 9}$ & $\mathbf{1 , 2 0}$ & $\mathbf{1 , 2 0}$ & $\mathbf{1 , 2 1}$ \\
\hline $\mathbf{8 . 5 2}$ & $\mathbf{1 , 5 0}$ & $\mathbf{1 , 0 5}$ & $\mathbf{1 , 1 5}$ & $\mathbf{1 , 2 7}$ & $\mathbf{1 , 1 4}$ \\
\hline $\mathbf{8 . 5 3}$ & $\mathbf{1 , 4 2}$ & $\mathbf{1 , 0 9}$ & $\mathbf{1 , 1 4}$ & $\mathbf{1 , 2 4}$ & $\mathbf{1 , 1 2}$ \\
\hline $\mathbf{8 . 5 4}$ & $\mathbf{1 , 2 5}$ & $\mathbf{1 , 0 4}$ & $\mathbf{1 , 0 9}$ & $\mathbf{1 , 2 0}$ & $\mathbf{1 , 1 0}$ \\
\hline
\end{tabular}

Tabela 8.53: Dummy / Klein - Soma e subtração de matriz vezes escalar $(10 \leq n \leq 90)$

E dá uma oscilada quando as matrizes passam de 700 elementos.

\begin{tabular}{|c|c|c|c|c|c|}
\hline $\begin{array}{c}\text { Tempo Dummy } \\
\text { Tempo Klein }\end{array}$ & \multicolumn{5}{|c|}{ Número de elementos } \\
\hline Função & $\mathbf{1 0 0}$ & $\mathbf{3 0 0}$ & $\mathbf{5 0 0}$ & $\mathbf{7 0 0}$ & $\mathbf{9 0 0}$ \\
\hline $\mathbf{8 . 4 6}$ & 0,83 & 0,74 & 0,50 & 0,57 & 0,55 \\
\hline $\mathbf{8 . 4 7}$ & $\mathbf{1 , 0 1}$ & 0,97 & 0,71 & 0,72 & 0,68 \\
\hline $\mathbf{8 . 4 8}$ & $\mathbf{1 , 1 0}$ & $\mathbf{1 , 1 5}$ & 0,87 & 0,83 & 0,76 \\
\hline $\mathbf{8 . 4 9}$ & $\mathbf{1 , 1 6}$ & $\mathbf{1 , 2 6}$ & 0,98 & 0,87 & 0,82 \\
\hline $\mathbf{8 . 5 0}$ & $\mathbf{1 , 2 7}$ & $\mathbf{1 , 2 9}$ & $\mathbf{1 , 0 4}$ & 0,89 & 0,89 \\
\hline $\mathbf{8 . 5 1}$ & $\mathbf{1 , 1 7}$ & $\mathbf{1 , 2 7}$ & $\mathbf{1 , 0 8}$ & 0,90 & 0,92 \\
\hline $\mathbf{8 . 5 2}$ & $\mathbf{1 , 1 9}$ & $\mathbf{1 , 2 9}$ & $\mathbf{1 , 1 1}$ & 0,90 & 0,96 \\
\hline $\mathbf{8 . 5 3}$ & $\mathbf{1 , 1 9}$ & $\mathbf{1 , 3 2}$ & $\mathbf{1 , 1 3}$ & 0,92 & $\mathbf{1 , 0 1}$ \\
\hline $\mathbf{8 . 5 4}$ & $\mathbf{1 , 2 4}$ & $\mathbf{1 , 3 0}$ & $\mathbf{1 , 1 4}$ & 0,95 & $\mathbf{1 , 0 4}$ \\
\hline
\end{tabular}

Tabela 8.54: Dummy / Klein - Soma e subtração de matriz vezes escalar $(100 \leq n \leq 900)$

\subsection{Multiplicação de matrizes}

Por fim, efetuamos os testes para multiplicação de matrizes.

$$
\begin{gathered}
r e s p=a * b, \\
r e s p=a * b * c, \\
r e s p=a * b * c * d, \\
r e s p=a * b * c * d * e, \\
r e s p=a * b * c * d * e * f, \\
r e s p=a * b * c * d * e * f * g, \\
r e s p=a * b * c * d * e * f * g * h, \\
r e s p=a * b * c * d * e * f * g * h * i, \\
r e s p=a * b * c * d * e * f * g * h * i * j,
\end{gathered}
$$

onde resp, $a, b, c, d, e, f, g, h, i$ e $j$ são matrizes.

O código da biblioteca MKL e ATLAS são iguais:

cblas_dgemm(CblascolMajor, CblasNoTrans, CblasNoTrans, n, n, n, 1, data(a), $\mathrm{n}, \operatorname{data}(\mathrm{b}), \mathrm{n}, \mathrm{0}, \operatorname{data}(\operatorname{resp} 2), \mathrm{n})$; 


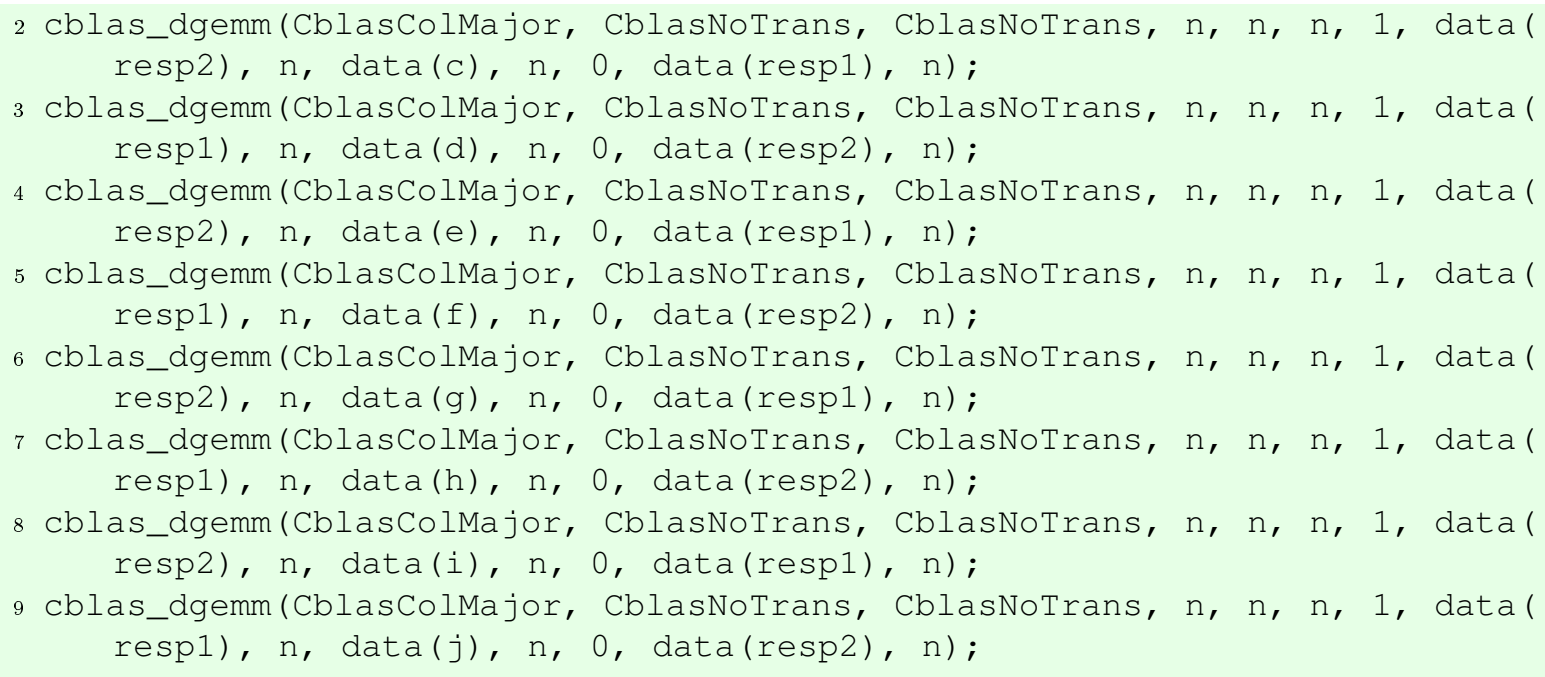

E a Klein realiza a multiplicação dessa outra maneira:

1 resp $=a \star b * c * d \star e \star f \star g * h \star i * j i$

\section{Atlas / Klein}

A Klein perde na maioria dos casos. Porém, o resultado era esperado, pois não houveram otimizações para a multiplicação de matrizes.

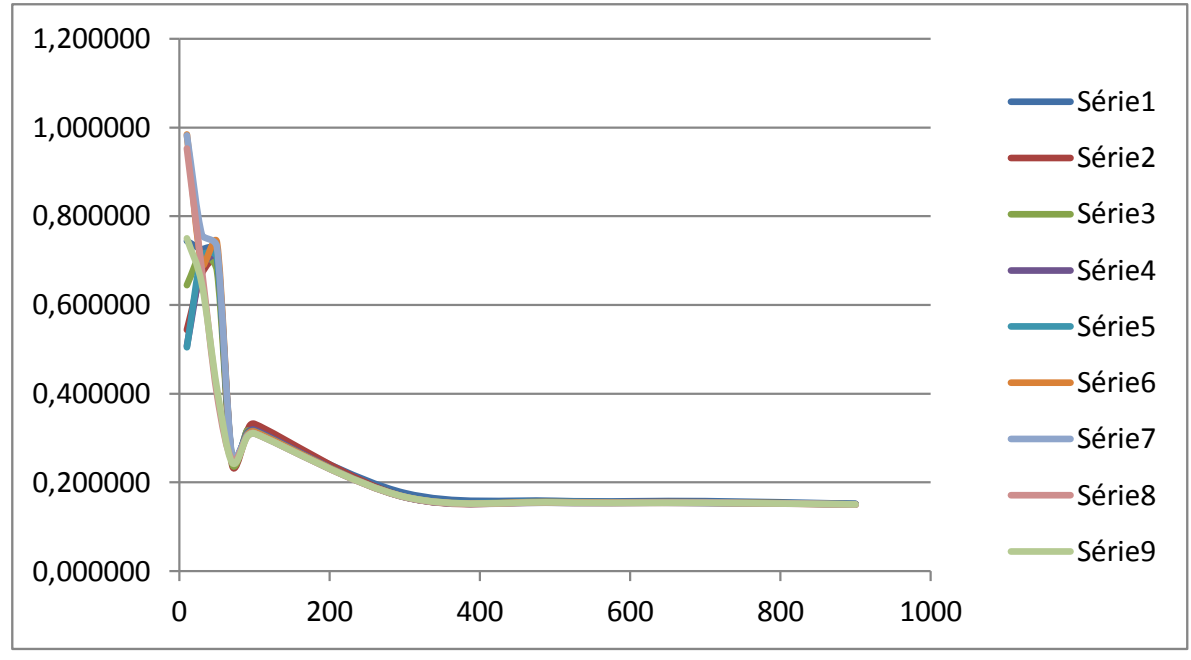

Figura 8.22: Multiplicação de matrizes - Atlas / Klein

Para matrizes com dimensão 10x10 a Klein chega a ser competitiva para os casos em que operamos sobre sete, oito ou nove matrizes. Porém a medida que aumentamos a dimensão das matrizes a Klein fica com desempenho bem inferior. 


\begin{tabular}{|c|c|c|c|c|c|}
\hline Tempo Atlas & \multicolumn{5}{|c|}{ Número de elementos } \\
\hline Tempo Klein & \multicolumn{1}{|c|}{} \\
\hline Função & $\mathbf{1 0}$ & $\mathbf{3 0}$ & $\mathbf{5 0}$ & $\mathbf{7 0}$ & $\mathbf{9 0}$ \\
\hline $\mathbf{8 . 5 5}$ & 0,74 & 0,73 & 0,72 & 0,25 & 0,32 \\
\hline $\mathbf{8 . 5 6}$ & 0,54 & 0,67 & 0,70 & 0,24 & 0,31 \\
\hline $\mathbf{8 . 5 7}$ & 0,64 & 0,72 & 0,68 & 0,24 & 0,32 \\
\hline $\mathbf{8 . 5 8}$ & 0,51 & 0,69 & 0,71 & 0,25 & 0,31 \\
\hline $\mathbf{8 . 5 9}$ & 0,50 & 0,71 & 0,71 & 0,26 & 0,31 \\
\hline $\mathbf{8 . 6 0}$ & 0,98 & 0,69 & 0,74 & 0,25 & 0,31 \\
\hline $\mathbf{8 . 6 1}$ & 0,98 & 0,76 & 0,73 & 0,26 & 0,30 \\
\hline $\mathbf{8 . 6 2}$ & 0,95 & 0,68 & 0,40 & 0,25 & 0,30 \\
\hline $\mathbf{8 . 6 3}$ & 0,75 & 0,65 & 0,41 & 0,24 & 0,30 \\
\hline
\end{tabular}

Tabela 8.55: Atlas / Klein - Multiplicação de matriz $(10 \leq n \leq 90)$

\begin{tabular}{|c|c|c|c|c|c|}
\hline$\frac{\text { Tempo Atlas }}{\text { Tempo Klein }}$ & \multicolumn{5}{|c|}{ Número de elementos } \\
\hline Função & 100 & 300 & 500 & 700 & 900 \\
\hline 8.55 & 0,32 & 0,18 & 0,16 & 0,16 & 0,15 \\
\hline 8.56 & 0,33 & 0,17 & 0,16 & 0,16 & 0,15 \\
\hline 8.57 & 0,31 & 0,17 & 0,16 & 0,16 & 0,15 \\
\hline 8.58 & 0,32 & 0,17 & 0,16 & 0,15 & 0,15 \\
\hline 8.59 & 0,32 & 0,17 & 0,16 & 0,15 & 0,15 \\
\hline 8.60 & 0,31 & 0,17 & 0,16 & 0,15 & 0,15 \\
\hline 8.61 & 0,31 & 0,17 & 0,15 & 0,15 & 0,15 \\
\hline 8.62 & 0,31 & 0,17 & 0,15 & 0,15 & 0,15 \\
\hline 8.63 & 0,31 & 0,17 & 0,15 & 0,15 & 0,15 \\
\hline
\end{tabular}

Tabela 8.56: Atlas / Klein - Multiplicação de matriz $(100 \leq n \leq 900)$

\section{MKL / Klein}

Ao compararmos com a MKL, a Klein fica com desempenho bem inferior. E a medida que aumentamos o número de elementos da matriz essa diferença aumenta.

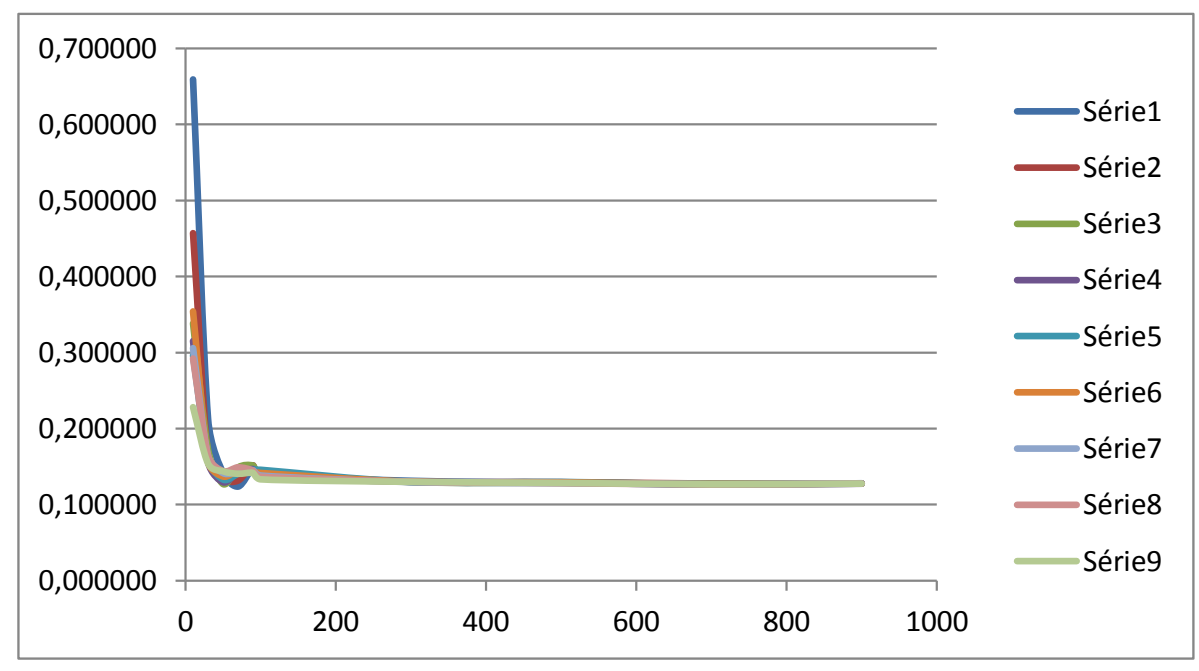

Figura 8.23: Multiplicação de matrizes - MKL / Klein 


\begin{tabular}{|c|c|c|c|c|c|}
\hline $\begin{array}{c}\text { Tempo MKL } \\
\text { Tempo Klein }\end{array}$ & \multicolumn{5}{|c|}{ Número de elementos } \\
\hline Função & $\mathbf{1 0}$ & $\mathbf{3 0}$ & $\mathbf{5 0}$ & $\mathbf{7 0}$ & $\mathbf{9 0}$ \\
\hline $\mathbf{8 . 5 5}$ & 0,66 & 0,21 & 0,14 & 0,12 & 0,15 \\
\hline $\mathbf{8 . 5 6}$ & 0,46 & 0,16 & 0,13 & 0,13 & 0,15 \\
\hline $\mathbf{8 . 5 7}$ & 0,34 & 0,17 & 0,13 & 0,15 & 0,15 \\
\hline $\mathbf{8 . 5 8}$ & 0,32 & 0,16 & 0,13 & 0,14 & 0,15 \\
\hline $\mathbf{8 . 5 9}$ & 0,30 & 0,16 & 0,13 & 0,14 & 0,15 \\
\hline $\mathbf{8 . 6 0}$ & 0,35 & 0,16 & 0,14 & 0,14 & 0,14 \\
\hline $\mathbf{8 . 6 1}$ & 0,31 & 0,16 & 0,14 & 0,14 & 0,15 \\
\hline $\mathbf{8 . 6 2}$ & 0,29 & 0,16 & 0,14 & 0,15 & 0,14 \\
\hline $\mathbf{8 . 6 3}$ & 0,23 & 0,15 & 0,14 & 0,14 & 0,14 \\
\hline
\end{tabular}

Tabela 8.57: $M K L /$ Klein - Multiplicação de matriz $(10 \leq n \leq 90)$

\begin{tabular}{|c|c|c|c|c|c|}
\hline $\begin{array}{c}\text { Tempo MKL } \\
\text { Tempo Klein }\end{array}$ & \multicolumn{5}{|c|}{ Número de elementos } \\
\hline Função & $\mathbf{1 0 0}$ & $\mathbf{3 0 0}$ & $\mathbf{5 0 0}$ & $\mathbf{7 0 0}$ & $\mathbf{9 0 0}$ \\
\hline $\mathbf{8 . 5 5}$ & 0,14 & 0,13 & 0,13 & 0,13 & 0,13 \\
\hline $\mathbf{8 . 5 6}$ & 0,14 & 0,13 & 0,13 & 0,13 & 0,13 \\
\hline $\mathbf{8 . 5 7}$ & 0,14 & 0,13 & 0,13 & 0,13 & 0,13 \\
\hline $\mathbf{8 . 5 8}$ & 0,14 & 0,13 & 0,13 & 0,13 & 0,13 \\
\hline $\mathbf{8 . 5 9}$ & 0,15 & 0,13 & 0,13 & 0,13 & 0,13 \\
\hline $\mathbf{8 . 6 0}$ & 0,14 & 0,13 & 0,13 & 0,13 & 0,13 \\
\hline $\mathbf{8 . 6 1}$ & 0,14 & 0,13 & 0,13 & 0,13 & 0,13 \\
\hline $\mathbf{8 . 6 2}$ & 0,14 & 0,13 & 0,13 & 0,13 & 0,13 \\
\hline $\mathbf{8 . 6 3}$ & 0,13 & 0,13 & 0,13 & 0,13 & 0,13 \\
\hline
\end{tabular}

Tabela 8.58: $M K L /$ Klein - Multiplicação de matriz $(100 \leq n \leq 900)$ 



\section{Capítulo 9}

\section{Testes genéricos}

Quando lidamos com bibliotecas genéricas, não basta criarmos testes de corretude para os tipos primitivos da linguagem. Os testes devem ser genéricos para permitir que novos tipos sejam adicionados de forma simples e rápida. Nesta seção, descrevemos um exemplo desses testes, cujo modelo foi seguido para todos os testes da Klein, e introduzimos alguns fundamentos teóricos para entendê-los melhor. Os testes foram criados utilizando o Framework Google C++ Test [4].

\subsection{Exemplo de teste genérico na Klein}

Tomando inicialmente um exemplo simples como a expressão $a=b+c$, onde $a, b$ e $c$ são vetores. Teríamos o seguinte código a ser testado:

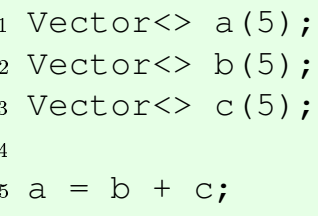

Para efetuar esse teste, a Klein utiliza-se da estrutura Binary_op_test, que é a responsável por invocar os testes de operações binárias, seja soma, multiplicação ou divisão. Isso é feito através da função check, que recebe como parâmetro três fábricas e um boolean, que retornará o resultado dos testes. Como trata-se de uma função template o mesmo código é utilizado para teste de matrizes, views e a combinação entre essas estruturas.

Porém, como a Klein utiliza-se da estratégia lazy para avaliação de expressões, poderíamos ter operações complexas em ambos os lados da operação. Portanto, para facilitar os testes, foram criadas as fábricas, que são estruturas capazes de gerar valores para uma estrutura específica.

Abaixo encontra-se o código da função check que faz a verificação se uma operação está correta na Klein.

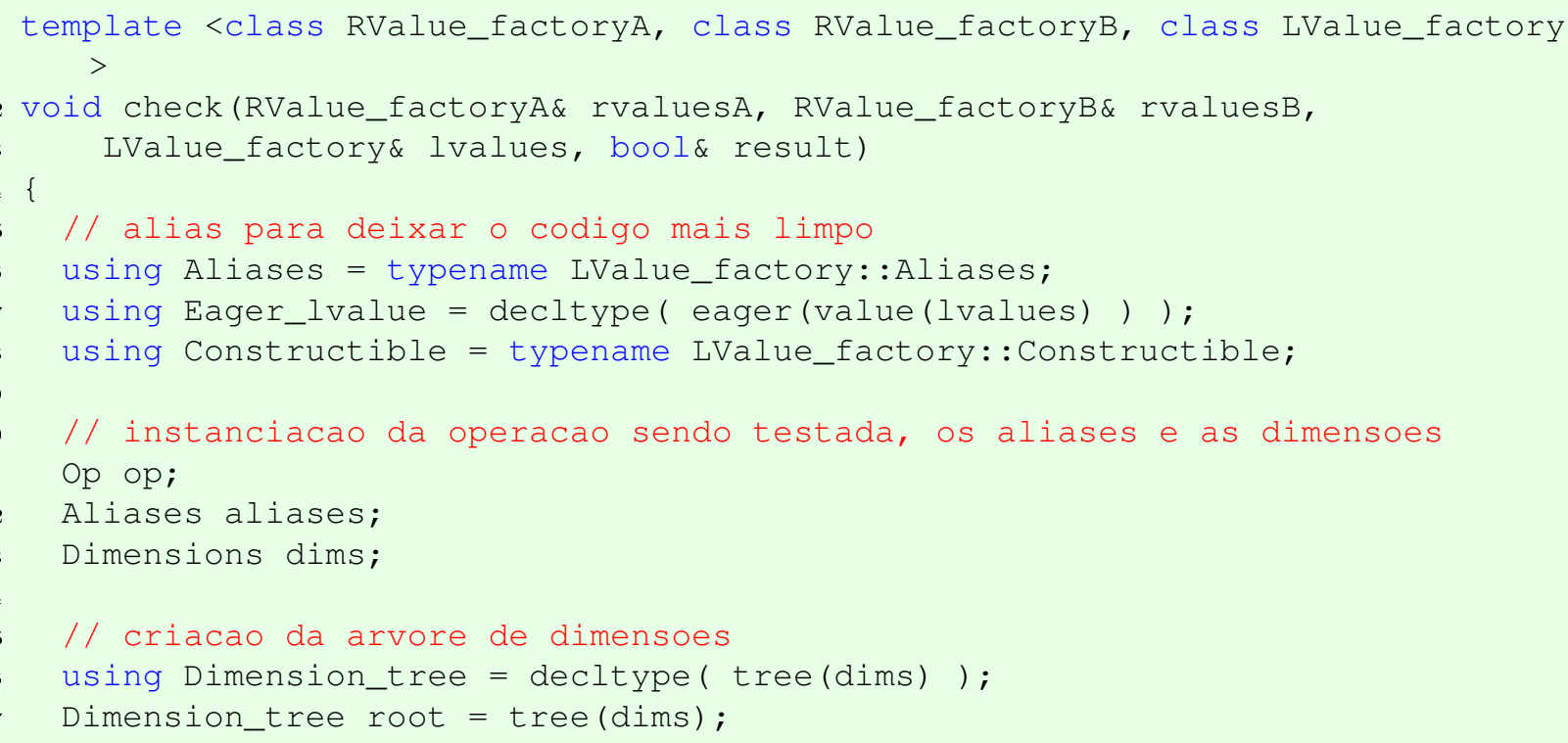




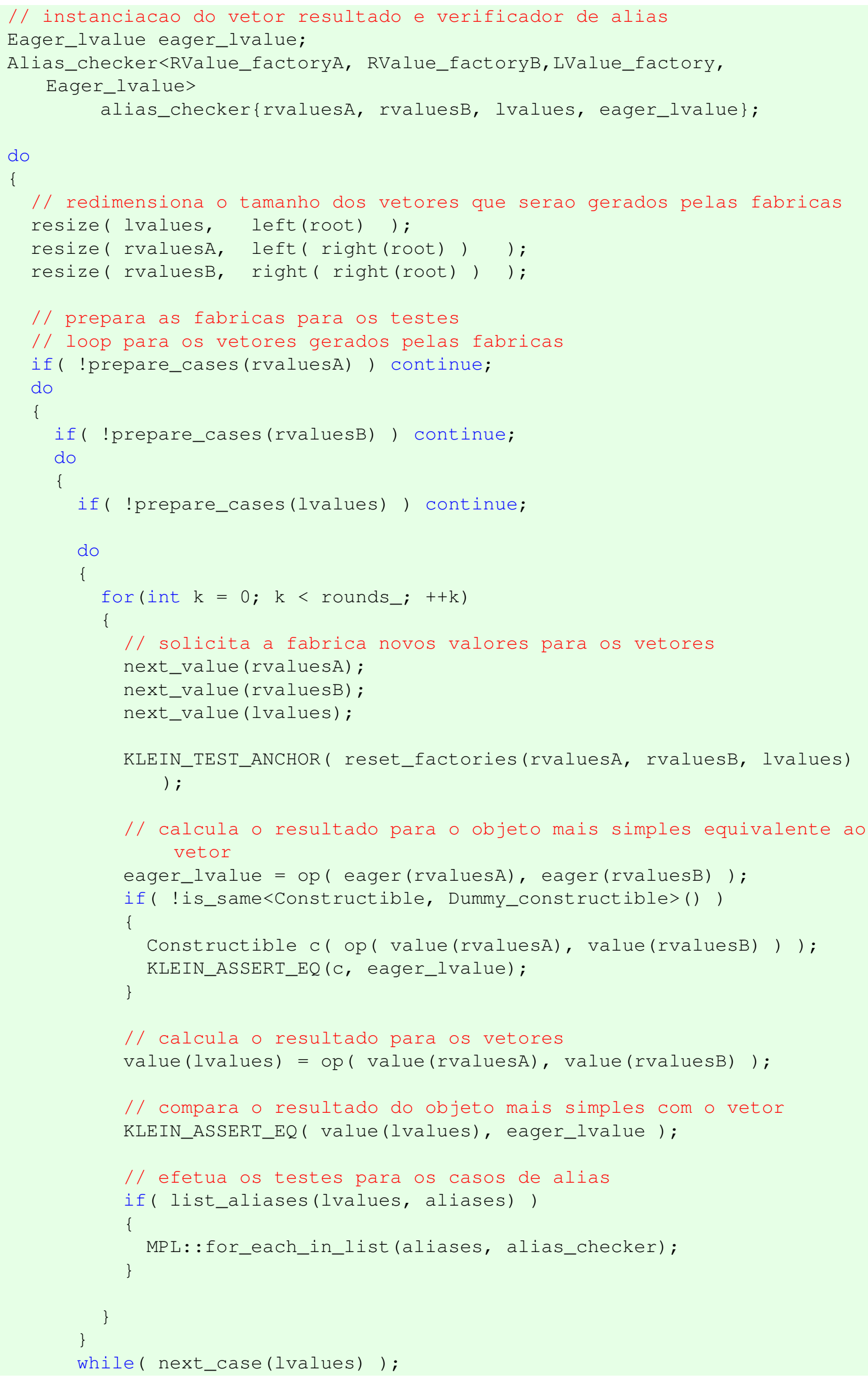




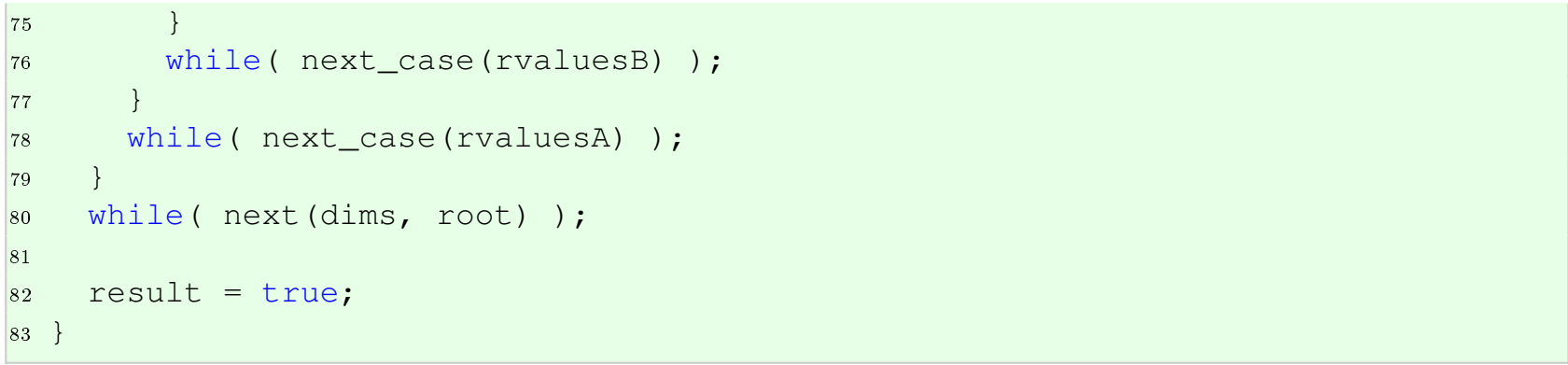

Nesse código, o primeiro passo é criar a árvore de dimensões. Conforme visto anteriormente, quando efetuamos operações entre objetos na Klein é criada uma árvore que representa essa expressão. Por exemplo, para a expressão $a=b+c$, onde $a, b$ e $c$ são matrizes, teríamos a árvore abaixo:

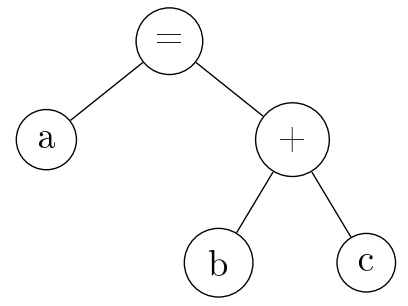

A dimensão das matrizes serão geradas com o número de colunas de a igual ao número de linhas de $b$. A matriz $c$ deve ter o número de linhas de $a$ e o número de colunas de $b$. O papel de gerar essas dimensões são de responsabilidade da estrutura Dimensions.

1 using Dimension_tree = decltype( tree(dims) );

2 Dimension_tree root $=$ tree (dims);

Por esse motivo, quando vamos definir as dimensões dos objetos, temos que percorrer a árvore fazendo as atribuições. Voltando ao exemplo de soma de vetores, o código fica da seguinte forma:

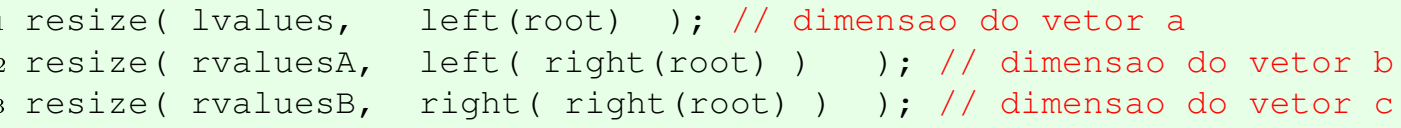

Ou seja, primeiro vamos para a esquerda, partindo da raiz, acessamos assim a dimensão gerada para o vetor a. O segundo passo é ir para a direita da raiz e depois esquerda, acessando a dimensão gerada para o vetor b. E por último seguindo direita e direita acessamos a dimensão de c. Por se tratar de uma estrutura para operações binárias, ou seja, entre dois elementos esse sempre será o caminho a ser percorrido na árvore.

O próximo passo do teste é preparar as fábricas para aquelas dimensões.

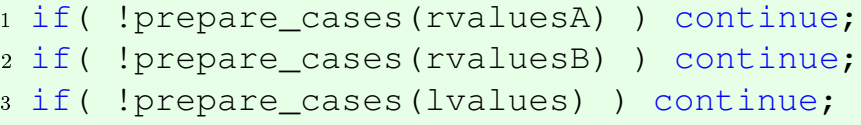

O terceiro passo é gerar valores para as estruturas de cada uma das fábricas.

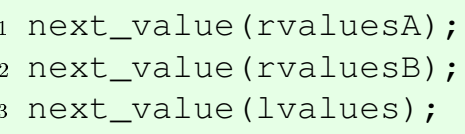

No código do teste podemos notar que isso é feito dentro de um laço. Isso ocorre, porque cada fábrica irá gerar novos valores aleatoriamente para os vetores.

Depois disso, a operação é realizada para o objeto mais simples equivalente ao vetor e para os objetos que queremos testar.

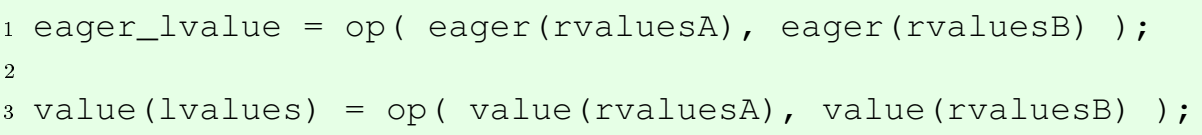


Por último, comparamos os resultados desses objetos. Caso sejam iguais, o teste está correto.

1 KLEIN_ASSERT_EQ( value(lvalues), eager_lvalue );

Para que o teste faça sentido é necessário garantirmos previamente que para o objeto mais simples equivalente ao vetor a operação está funcionando.

\subsection{Gerador de testes na Klein}

Para um exemplo parecido com o da seção anterior, mas ao invés de somar dois vetores quiséssemos somar um vetor com uma view:

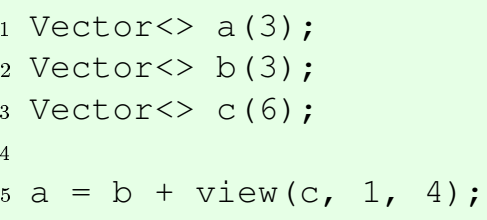

o que mudaria nos testes? E se o interesse fosse esse outro código?

$1 \mathrm{a}=\operatorname{view}(\mathrm{c}, 1,4)+\mathrm{c}$;

Nos códigos vistos até agora, nada mudaria. Foram implementadas na Klein estruturas para que coisas desse tipo fossem tratadas de forma genérica. Mas além disso, para que as combinações entre estruturas diferentes também fossem todas cobertas. Nesta seção vamos descrever um exemplo de teste e as estruturas utilizadas por ele.

O exemplo que descreveremos será o teste soma de vetores, cujo código encontra-se abaixo. O primeiro passo, é a criação das listas de fábricas de vetores e views.

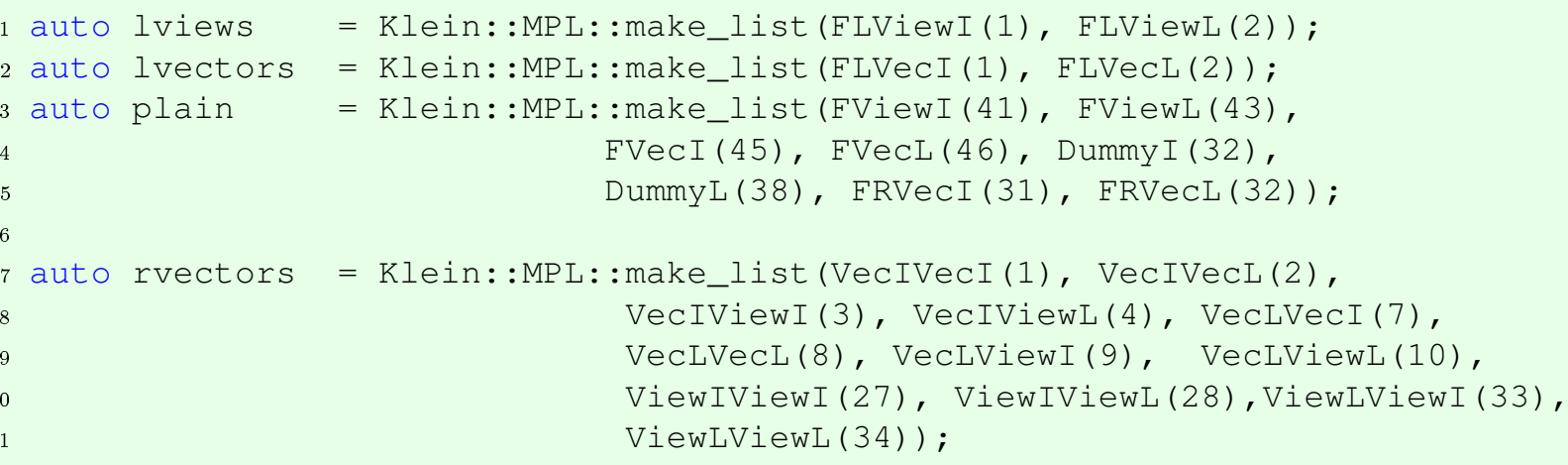

As listas lviews contém as fábricas de lvalues views, lvectors contém as fábricas de lvalues vectors, plain contém fábricas de views e vectors, e rvectors contém as fábricas de rvalues views e rvalues vectors.

No próximo passo, instanciamos o teste de operações binárias, informando a função que desejamos testar e as dimensões dos vetores.

1 Klein::Tests: :Binary_op_test<Plus, Dim_xy $2<$ Diml, Dim_x_copies_y $<$ DimT $>>>$ test_a;

Por último, invocamos a função for_each_in_lists que irá gerar os códigos da combinação entre todos os elementos das listas passadas como parâmetro.

1 Klein::MPL: :for_each_in_lists(plain, rvectors, lvectors, test_a);

O código fica da seguinte maneira:

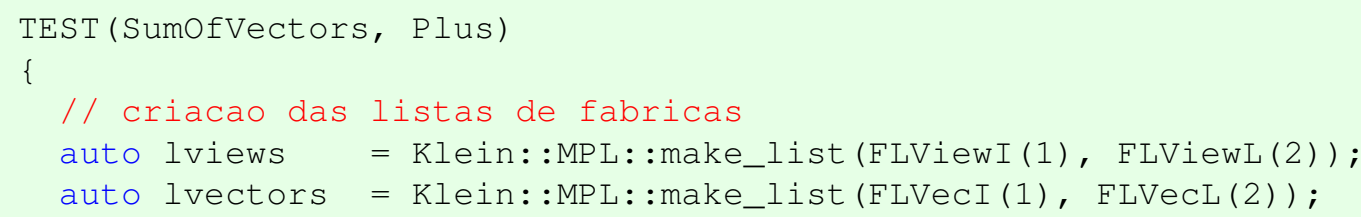




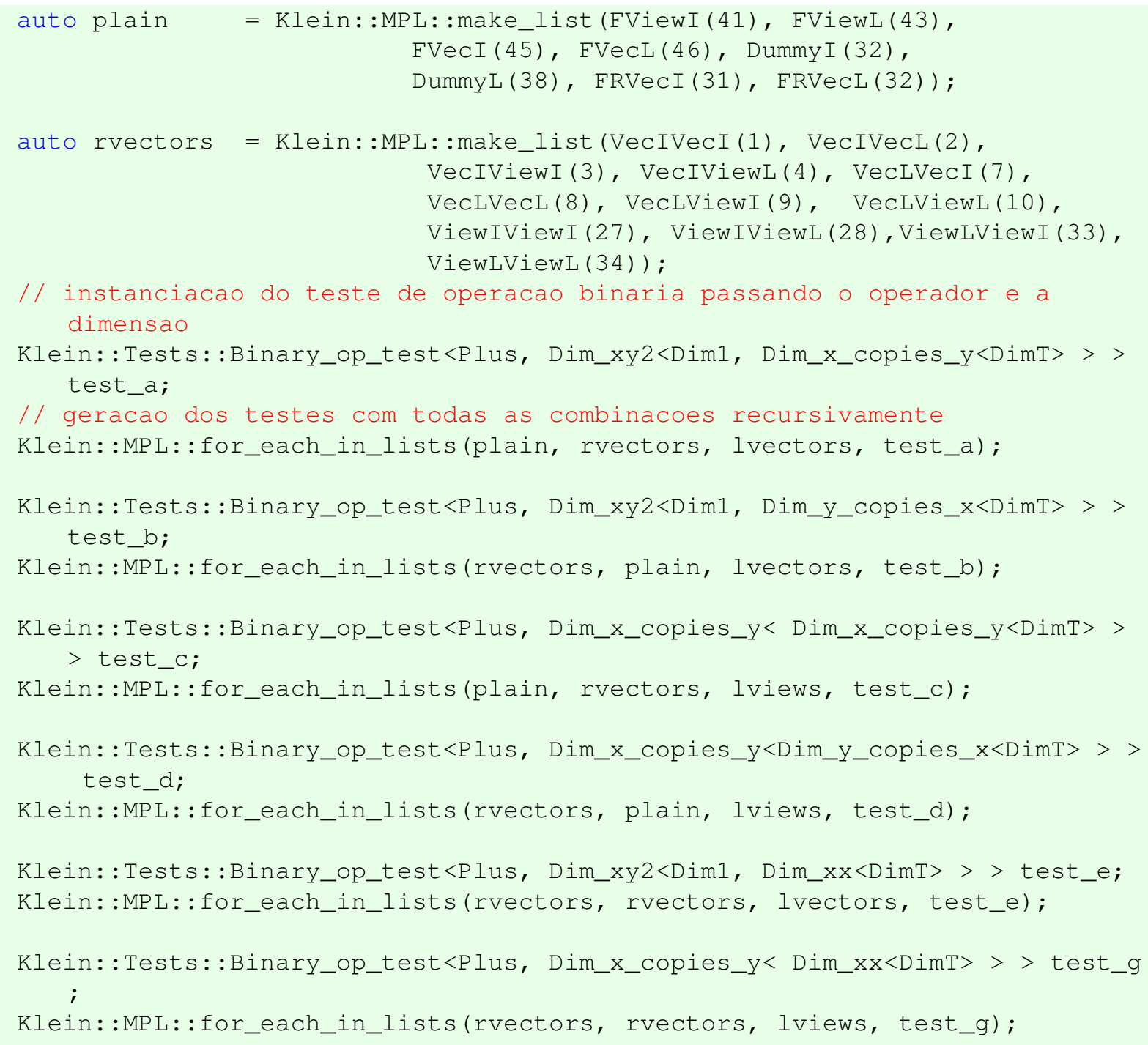

Primeiro, instanciamos as fábricas. Essas são as responsáveis por retornar estruturas de dados preenchidas com o tipo numérico passado no parâmetro template. Depois, instanciamos as listas de fábricas. Essas listas são basicamente divididas em lvalue, que são as estruturas que podem estar tanto do lado direito, quanto esquerdo da operação sob teste, e rvalue, que são as estruturas que estarão obrigatoriamente do lado direito da operação. Na sequência, a função teste é instanciada, passando, nos parâmetros templates, a função que será testada e as dimensões das estruturas de dados. Por último, invocamos o teste passando as listas de fábricas como parâmetro. Nesse momento serão criados códigos para todas as combinações possíveis entre as listas de fábricas, em tempo de compilação.

Antes de explicar cada passo do teste, vamos entender alguns conceitos e como funcionam as estruturas de geração de testes da Klein.

\section{lvalue e rvalue}

Um lvalue representa um objeto que ocupa um lugar identificável na memória, caso contrário, será um rvalue [11].

Para entender, vejamos alguns exempos simples.

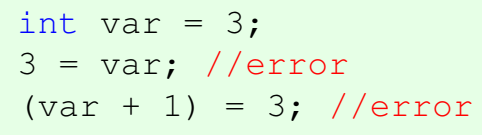

Uma atribuição espera um lvalue como o operando da esquerda. Como var é um objeto com 
posição de memória identificável, a primeira linha está correta. Porém nem a constante 3 nem a expressão $(\operatorname{var}+1)$ são lvalues, pois ambos são resultados de expressões que estarão temporariamente na memória. Portanto, fazer uma atribuição para qualquer um deles está errado.

Outro exemplo dessa diferença é o código abaixo:

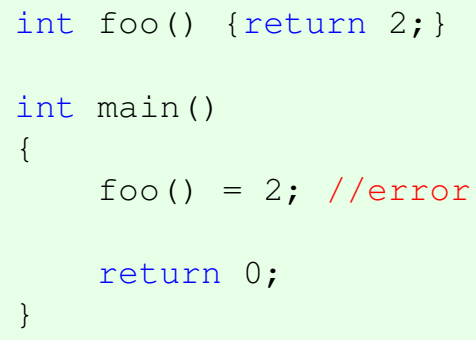

O retorno da função foo() é um rvalue de valor 2, que não possui um endereço identificável na memória. Ele é um temporário que será destruído assim que a função retornar. O código está errado, por causa da atribuição na função foo(). Se alterarmos a função foo() conforme abaixo:

1 int\& foo() \{return 2;

ainda continuará errado, pois estamos retornando uma referencia para um valor cujo endereço não é identificável.

Porém nem toda atribuição ao retorno de uma função é inválida. Por exemplo, o código abaixo está correto.

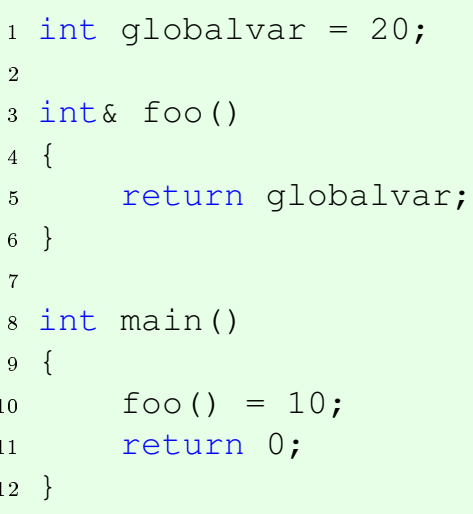

A função foo() retorna uma referência para um lvalue, ou seja, retorna uma referência para uma posição identificável da memória. Por isso, a atribuição é possível nesse caso.

\section{Fábrica de estruturas}

Uma fábrica recebe como parâmetro uma semente para geração dos valores aleatórios, como parâmetro template recebe uma fábrica de escalar, é capaz de se redimensionar, gerar novos valores para a estrutura, preparar casos de alias, gerar novos casos de alias e retornar essa estrutura no formato mais simples.

Por exemplo, uma fábrica de views rvalues possui as funções reset, resize, next_value, prepare_cases, prepare_aliases, next_alias e eager.

- reset: reinicializa os valores da fábrica de escalar.

- resize: redimensiona o vetor.

- next_value: gera novos valores para o vetor.

- prepare_cases: prepara a fábrica para gerar os casos de teste.

- prepare_aliases: prepara a fábrica para gerar os casos de alias.

- next_alias: gera novos casos de alias. 
- eager: retorna o objeto mais simples equivalente à view, que no caso é um vetor. A forma mais simples para um vetor, por exemplo, também é um vetor.

O código dessa estrutura fica conforme abaixo:

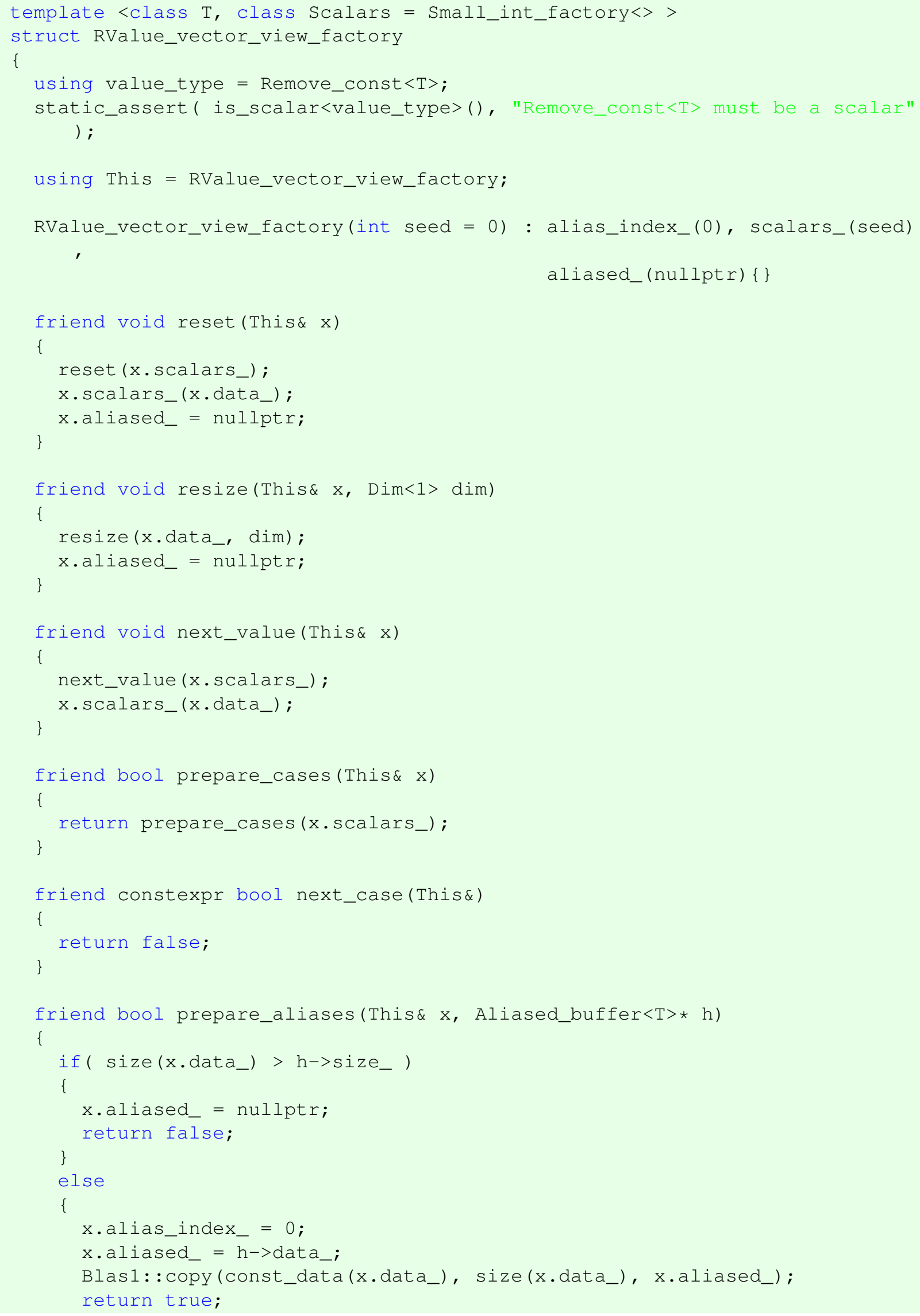




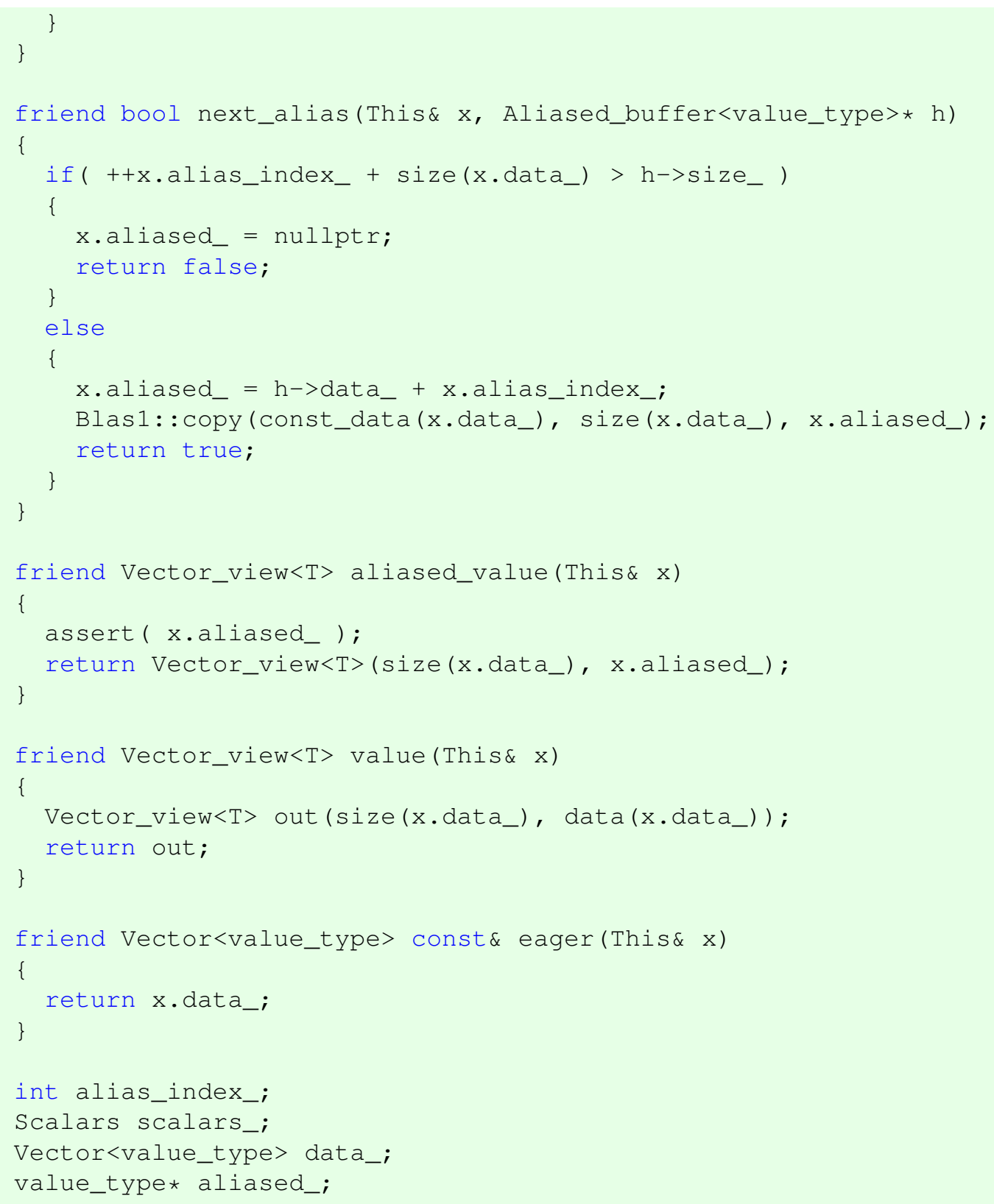

As fábricas de todas as estrutura usadas nos testes da Klein possuem esse mesmo padrão, possibilitando o polimorfismo em tempo de compilação.

\section{make_list}

A função make_list monta uma lista com os objetos passados como parâmetro.

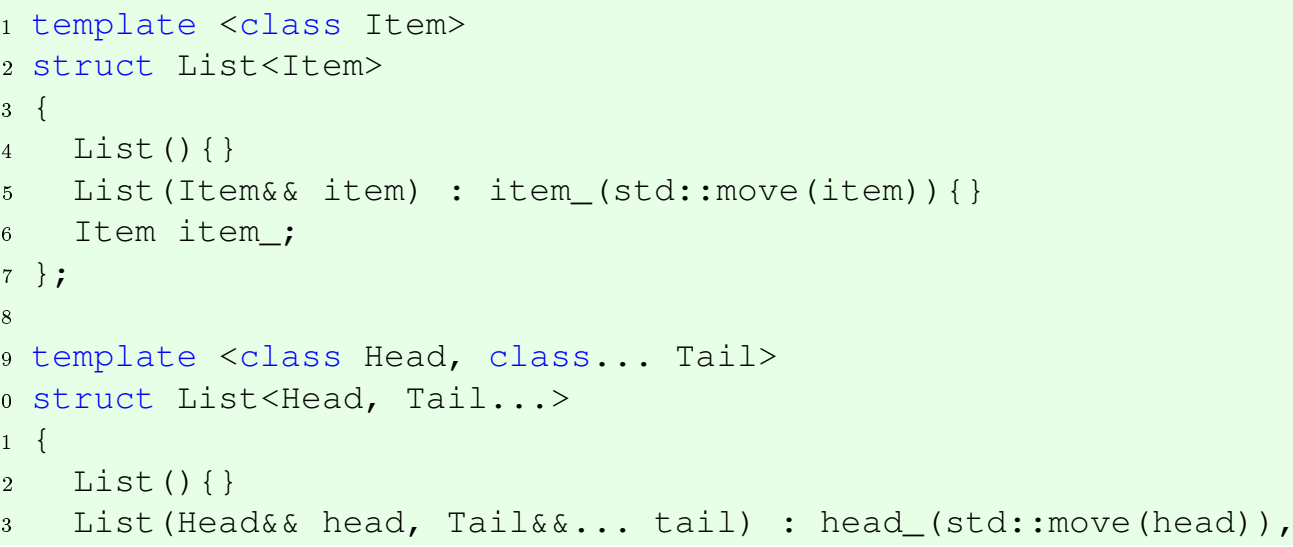




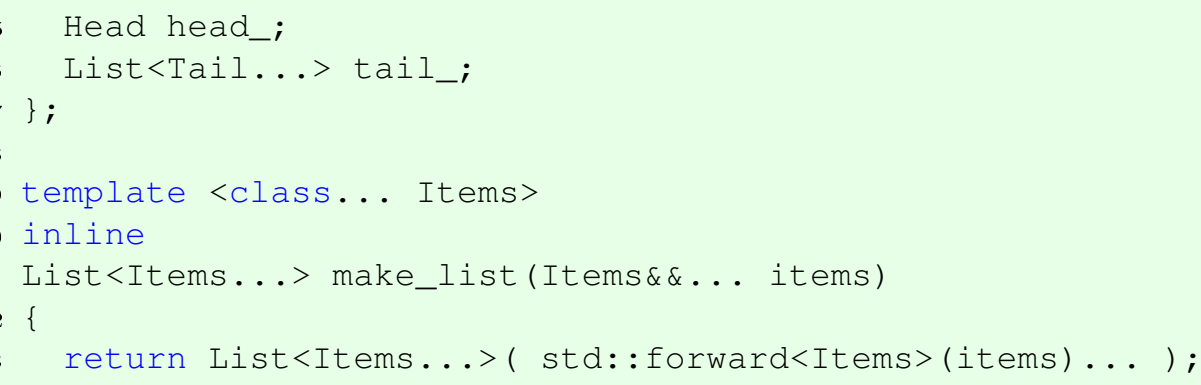

A lista contém o elemento da cabeça, e outra lista com todos os outros elementos, que por sua vez também possui a mesma estrutura. Ou seja, para uma lista contendo os elementos a, b, c, d teríamos uma estrutura como esta:

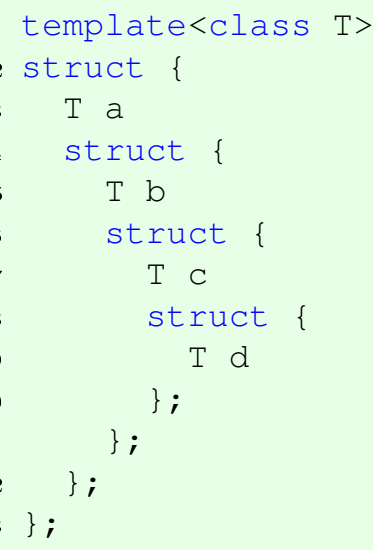

Como a lista é criada através dos parâmetros templates, o código é gerado em tempo de compilação.

\section{Binary_op_test}

Conforme visto na seção anterior, a estrutura Binary_op_test é a responsável pela execução do teste. Ela contém a sobrecarga do operador parênteses, que é o responsável por invocar os testes através do método check.

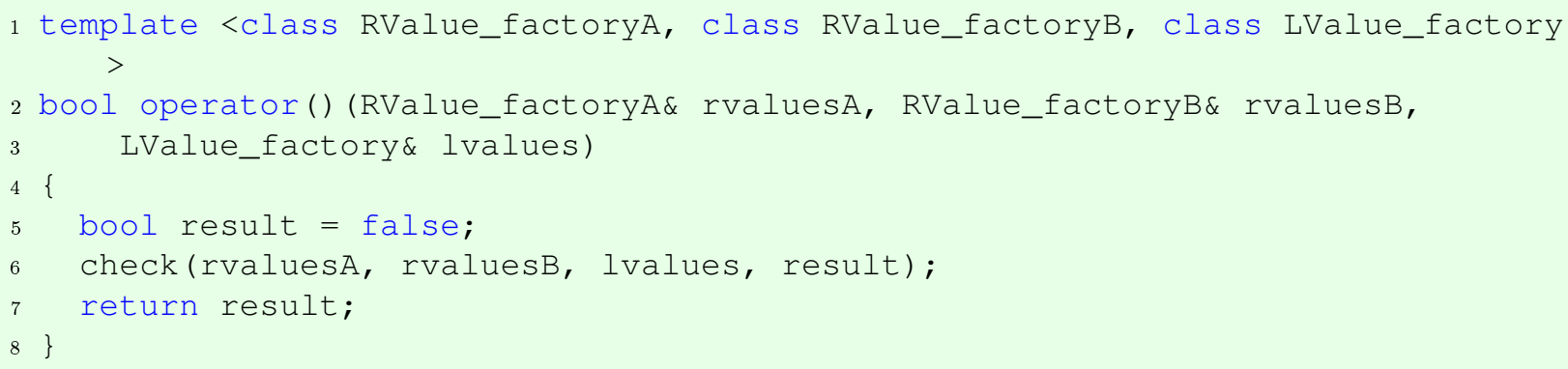

Esse ponto em diante foi descrito na seção anterior.

\section{for_each_in_lists}

for_each_in_lists é uma metafunção, que gera em tempo de compilação todas as combinações entre os elementos da lista $x s$, com os elementos da lista ys e com os elementos da lista $z s$.

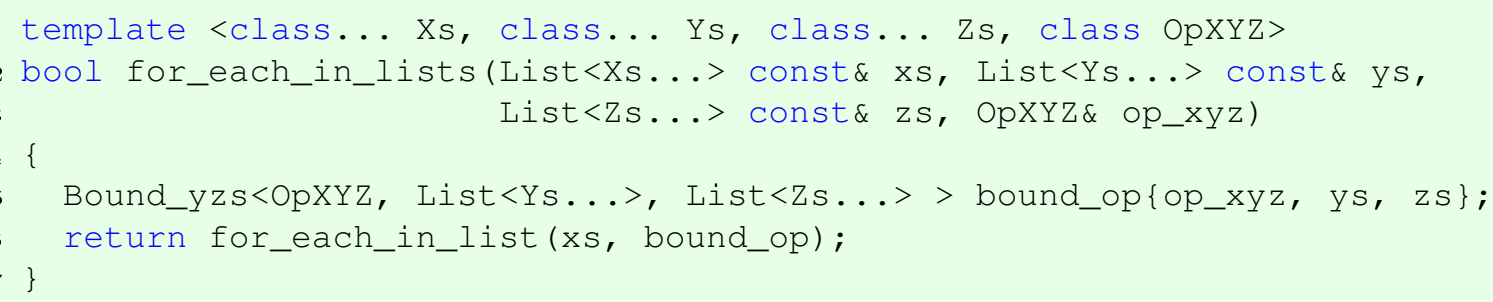


Ao instanciar o objeto Bound_yzs a estrutura abaixo será criada.

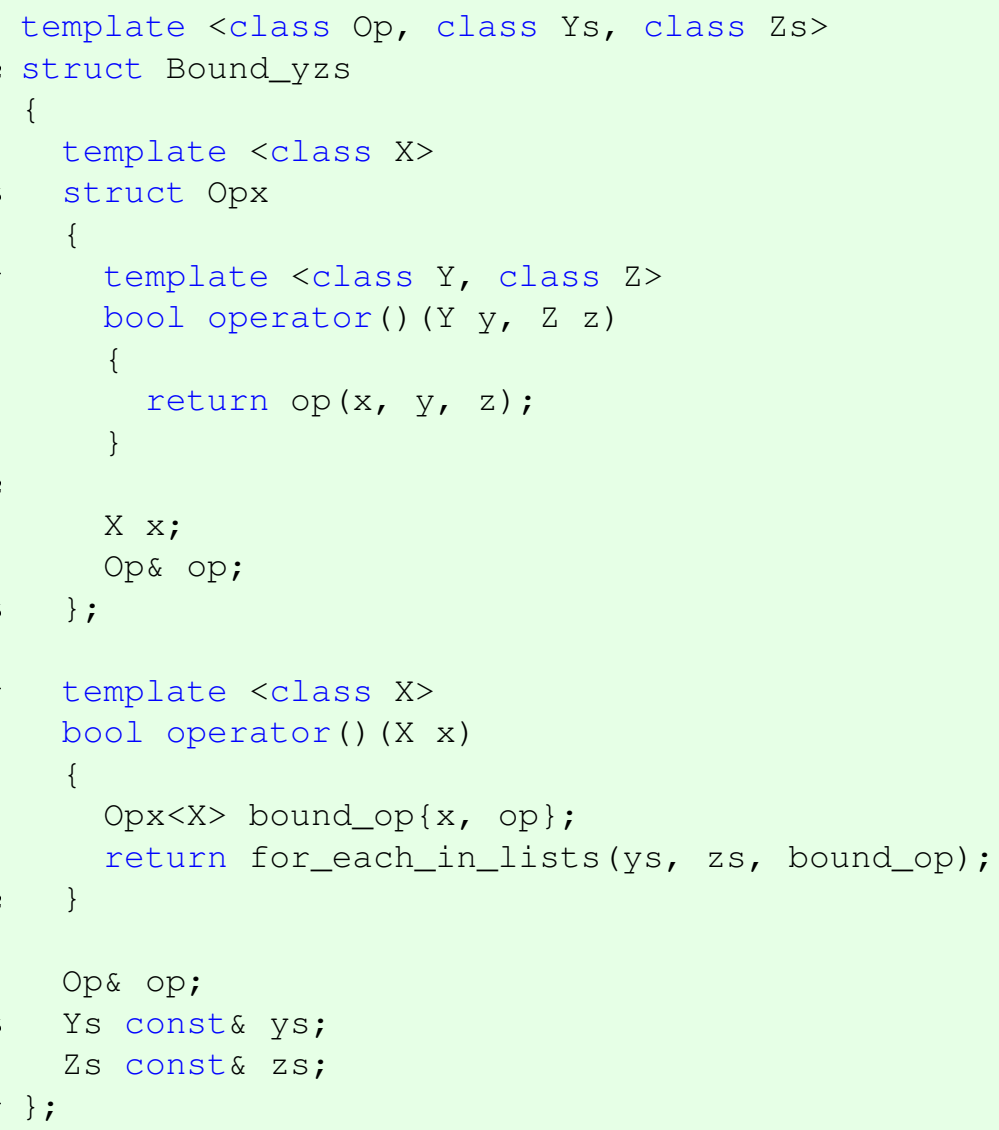

A função for_each_in_list(xs, bound_op) será invocada com uma nova assinatura

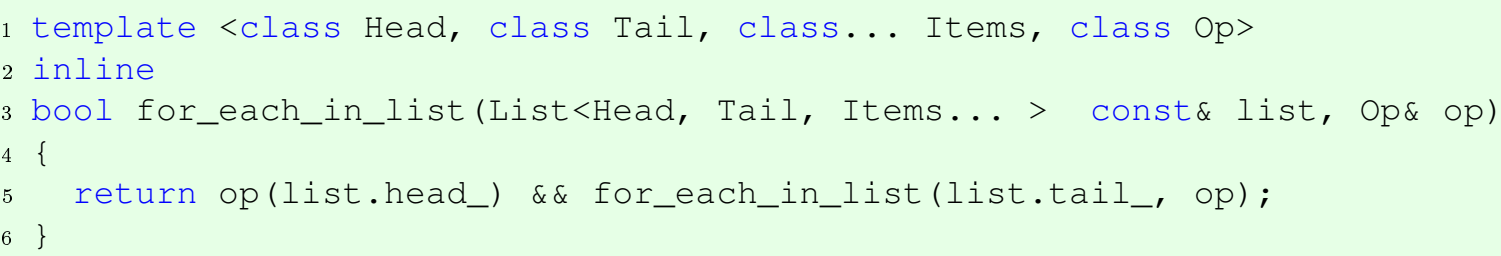

o objeto op possui sobrecarga do operador parenteses. O comportamento desse operador será a criação do objeto $O p x$, que efetua a operação entre os primeiros elementos de cada lista. E a invocação do método for_each_in_lists novamente, porém com outra assinatura, que recebe apenas duas listas e um operador.

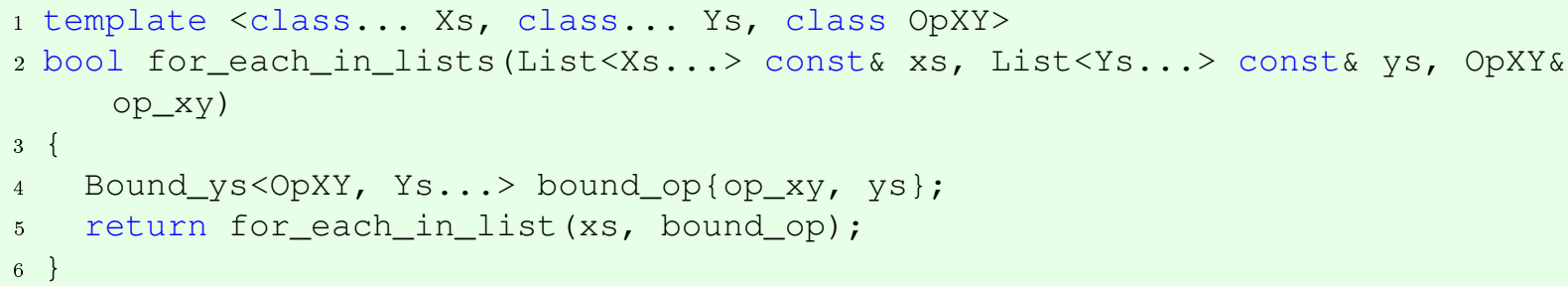

Essa assinatura instancia a estrutura Bound_ys:

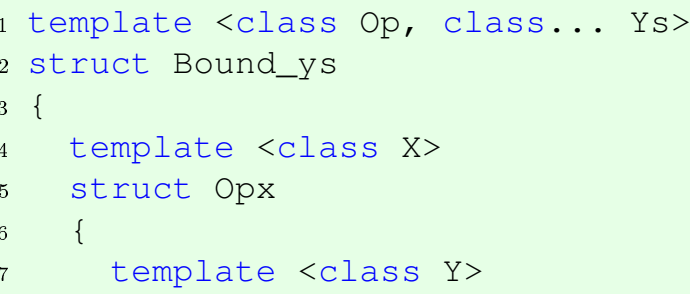


- FViewL é uma fábrica de views rvalue que contém elementos do tipo long.

- FVecI é uma fábrica de vetores de elementos do tipo int.

- FVecL é uma fábrica de vetores de elementos do tipo long.

- DummyI é uma fábrica de vetores de elementos do tipo int.

- DummyL é uma fábrica de vetores de elementos do tipo long.

- FRVecI é uma fábrica de referência para vetores de elementos do tipo int.

- FRVecL é uma fábrica de referência para vetores de elementos do tipo long.

- VecIVecI é uma fábrica de soma de vetores com elementos do tipo int.

- VecIVecL é uma fábrica de soma de vetores, sendo um com elementos do tipo int e outro do tipo long.

- VecIViewI é uma fábrica de soma entre vetor com elementos do tipo int e view com elementos do tipo int.

- VecIViewL é uma fábrica de soma entre vetor com elementos do tipo int e view com elementos do tipo long.

- VecLVecI é uma fábrica de soma entre vetor com elementos do tipo long e vetor com elementos do tipo int.

- VecLVecL é uma fábrica de soma entre vetores com elementos do tipo long.

- VecLViewI é uma fábrica de soma entre vetor com elementos do tipo long e view com elementos do tipo int.

- VecLViewL é uma fábrica de soma entre vetor e view com elementos do tipo long.

- ViewIViewI é uma fábrica de soma entre views com elementos do tipo int.

- ViewIViewL é uma fábrica de soma entre view com elementos do tipo int e view com elementos do tipo long.

- ViewLViewI é uma fábrica de soma entre view com elementos do tipo long e view com elementos do tipo int.

- ViewLViewL é uma fábrica de soma entre views com elementos do tipo long.

Outro fator importante é que para adicionarmos um teste para um novo tipo numérico, por exemplo, bastaríamos acrescentar a fábrica desse tipo nas listas criadas. 


\section{Capítulo 10}

\section{Conclusões}

Havia duas possibilidades para se criar a biblioteca Klein. Uma através de programação orientada a objetos e outra por meio de programação genérica. A programação orientada a objetos talvez fosse mais fácil, visto que erros oriundos dela são mais amigáveis em $\mathrm{C}++$. Esse foi, por exemplo o caminho seguido por bibliotecas como Eigen[1] e Armadillo[2]. Porém na abordagem orientada a objetos as chamadas de funções podem ser resolvidas em tempo de execução, o que afeta o desempenho do software, além de impedir que o compilador otimize o código sendo compilado. Enquanto isso, a programação genérica, desenvolvida em $\mathrm{C}++$ através de templates, pode resolver as chamadas de funções em tempo de compilação, além de criar funções inline que podem ser otimizadas pelo compilador.

Ao criar a biblioteca utilizando programação genérica, optou-se pelo melhor desempenho, visto que a Klein resolve todas as chamadas de funções em tempo de compilação. O que significaria que a biblioteca deveria ter um desempenho comparável com outras bibliotecas de mesmo propósito. Esse objetivo foi parcialmente atingido, conforme verificamos nos testes do capítulo 8, onde comparamos o desempenho da Klein com as bibliotecas otimizadas ATLAS e $M K L$, além de estratégias de cálculo como expression templates e dummy. O desempenho da Klein foi muito bom em alguns casos, porém ruim em outros, o que nos sugere que a biblioteca ainda precisa evoluir.

Nos testes de desempenho criamos vários códigos para cada uma das bibliotecas e estratégias. Ficou evidente que o código da Klein para o cálculo de expressões fica mais intuitivo que os demais. Também pudemos observar que a Klein é mais rápida para criar novos algoritmos, pelo menos para os tipos primitivos da linguagem e para os tipos já existentes na Klein.

O maior ponto negativo da Klein é a dificuldade do uso de templates em $\mathrm{C}++$. Mesmo para erros simples, $\mathrm{C}++$ pode gerar várias páginas de mensagens de erro, nada amigáveis. Isso pode fazer com que usuários inexperientes desistam rapidamente de utilizar a biblioteca. Porém ainda há esperança. Está em desenvolvimento para o $\mathrm{C}++14$ [10] um recurso chamado concepts, que promete mensagens de erro compreensíveis.

Apesar disso, a Klein ainda pode ser útil para teste de algoritmos. Podemos, por exemplo, criar algoritmos para solução de problemas lineares ou de otimização. E depois de comprovar que o algoritmo funciona, verificar se o desempenho é suficiente. Caso não seja, poderíamos traduzir o código para outra linguagem ou biblioteca, ou criar wrappers na Klein. A vantagem seria identificar que o algoritmo não funciona antes de despender um tempo enorme no seu desenvolvimento.

Esperamos que além dos tipos padrão do $\mathrm{C}++$, como float e double, que são úteis nas situações mais comuns, a Klein seja utilizada com outros tipos de aritmética, como a racional, intervalar ou de precisão estendida. O objetivo principal dessa primeira versão era ter algo funcional para que as pessoas fossem utilizando e identificando melhorias a serem implementadas em novas versões.

\subsection{Sugestões para Pesquisas Futuras}

A primeira sugestão para trabalhos futuros na Klein, é simplificar o que já existe. Como trata-se de uma biblioteca com alto nível de abstração, o código precisa de retoques para ficar mais fácil de entender e atualizar. Em conjunto com esse passo, melhorias seriam implementadas, tanto do ponto de vista de organização, quanto de desempenho. Nesse sentido teríamos a criação de wrappers para bibliotecas otimizadas, ou para programação multithread, tanto em CPU quanto em GPU. Tendo ao final desses passos uma biblioteca mais robusta. 
Outro aspecto importante a ser explorado é a utilização de concepts. concepts é um novo recurso da linguagem $\mathrm{C}++$ que estará disponível a partir do $\mathrm{C}++14$, que extinguirá as várias páginas de mensagens de erro ao utilizar templates. Talvez seja necessário reescrever a biblioteca, mas se conseguirmos esconder do usuário final essas mensagens o uso desse recurso seria bastante promissor. 


\section{Referências Bibliográficas}

[1] API documentation Eigen3, 2010. http://eigen.tuxfamily.org/dox/. 121

[2] Armadillo: An Open Source C ++ Linear Algebra Library for Fast Prototyping and Computationally Intensive Experiments, 2010. http://arma.sourceforge.net/. 121

[3] Automatically Tuned Linear Algebra Software (ATLAS), 2013. http://math-atlas.sourceforge. net. 1,63

[4] Google C++ Testing Framework, 2013. http://code.google.com/p/googletest/. 109

[5] Andrei Alexandrescu. Modern $C++$ Design: Generic Programming and Design Patterns Applied. 2004. 3

[6] Kent Beck. Test Driven Development - By Example. 2004. 57

[7] J. M. Marins D. M. Claudio. Cálculo numérico computacional. 2005. 13

[8] INRIA et al. The GNU MPFR Library, 2000. 7

[9] Intel. Intel @ math kernel library - reference manual, 2013. http://software.intel.com/en-us/ intel-mkl. 1, 63

[10] Mark Nelson. The $c++14$ standard: What you need to know. 2014. 121

[11] Dan Saks. Lvalues and rvalues. 2001. 113

[12] B. Stroustrup. Adding classes to the c language: An exercise in language evolution software practice and experience. 1983. 3

[13] T. Veldhuizen. Expression templates. 1995. 63 


\section{Índice Remissivo}

Algoritmos da STL, 31

fill, 31

generate, 32

transform, 33

Algoritmos de Estatística, 34

are_near, 35

col_means, 37

col_std_dev, 37

dot, 36

frob_norm, 39

$\max , 34$

max_abs, 36

$\max$ dist, 36

mean, 37

min, 34

non_zeros, 38

std_dev, 37

sum, 35

two norm, 38

zscore, 38

Algoritmos de propósito geral, 39

copy_data, 40

cos, $\overline{41}$

floor, 40

$\sin , 40$

zero, 39

Alias, 14

Alias na Klein, 16

Blas1

abs, 41

add, 43

div, 43

dive, 43

dot, 42

mul, 44

mul_add, 44

mul_add_iz, 44

mul_iy, $\overline{44}$

mul_rev_sub, 45

mul_rev_sub_iz, 45

mul_sub, 45

mulc, 46

mulc_add, 46, 47 mulc_add_ix, 47

mulc add iy, 46

rev__divc, $\overline{4} 3$

sub, 42

sub_ix, 42

sub_iy, 42

sum, 41

Blas2

matrix_mul_vector_add, 47

matrix_mul_vector_add_iy, 48

matrix_mul_vector_rev_sub, 49

matrix_mul_vector_sub, 48

matrix_mul_vector_sub_iy, 48

matrix_x_vector, 47

transpose, 49

Blas3

matrix_mul_add, 50

matrix_mul_add_ic, 50

matrix mul rev sub, 51

matrix mul_rev sub ic, 51

matrix_mul_sub, 50

matrix_mul_sub_ic, 51

matrix_x_matrix, 49

matrix_x_transposed_matrix, 52

transposed_matrix_x_matrix, 52

Classes Traits, 18

integral_constant, 18

is fundamental, 18

is_same, 18

is_scalar, 18

Classes Traits na Klein

Exemplo, 20

is_lazy_matrix, 19

is_lazy_vector, 19

is_rvalue_matrix, 20

is_rvalue_vector, 19

Decomposições

LU, 29

QR, 29

Escalares

Interval, 22 
Real, 21

Estratégias para avaliar expressões

Comparação entre eager e lazy, 13

Eager, 12

Lazy na Klein, 13

Lazy sem uso de memória temporária, 12

Lazy usando expression template, 13

Expressões aritméticas

Matrizes, 24

Matrizes e views, 24

Vetores, 24

Vetores e views, 24

Vetores, matrizes e views, 25

Gerador de testes

Binary_op_test, 115

Exemplo de geração de testes, 110

Fábrica de estruturas, 112

for_each_in_lists, 115

lvalue e rvalue, 111

make_list, 114

Objetos da Klein

Algoritmos, 30

Decomposição LU, 29

Decomposição QR, 30

Decomposição SVD, 30

Decomposições, 29

Escalares, 21

Expressões aritméticas, 24

Matrizes, 22

Vetores, 22

Views de matrizes, 23

Views de vetores, 23

Operadores lineares

Matriz diagonal, 26

Permutação de linhas e colunas de matrizes, 25

Transformação de matriz em vetor, 28

Transformação de vetor em matriz diagonal, 28

Templates em $\mathrm{C}++$

Classes template, 5

Especialização de templates, 5

Funções template, 3

Tipos Genéricos de números, 6

Teste de Performance

Soma de vetor vezes escalar, 71

Teste de Software

Etapas de teste, 60

Teste caixa branca, 58

Teste caixa preta, 57
Testes de Desempenho

Soma de vetores, 63

Testes de Performance

Multiplicação de matrizes, 102

Soma de matriz vezes escalar, 91

Soma de matrizes, 87

Soma e subtração de matriz vezes escalar, 97

Soma e subtração de vetor vezes escalar, 79

Testes Genéricos

Exemplo de teste, 107 